\title{
Markov Chain Aggregation for Agent-Based Models
}

\author{
Dissertation \\ zur Erlangung des Doktorgrades \\ an der Fakultät für Physik \\ der Universität Bielefeld
}

vorgelegt von

Sven Banisch

Bielefeld 2013 

»Die Mathematik ist eine

Karikatur der Wirklichkeit «

(Philippe Blanchard) 



\section{Acknowledgements}

Science needs freedom. It needs free thought, it needs free time, it needs free talk. Philippe Blanchard is among those teachers who are deeply committed to such an understanding of science. I am very proud and grateful to be among his students.

I am also very grateful to Ricardo Lima. He is probably the person who engaged most in the details of this project and should really be an honorary member of the reading committee. Thank you for always inspiring discussions and the critical reading of all parts of this work.

Tanya Araújo has given me encouragement since the first day we met. She also read through all the thesis and her advises (especially during the last turbulent months) helped a lot to finalize the writing.

I was once told that those people who are most busy (those that really are and do not just pretend to be) are also the people who always have time when you approach them with some question or meet them on the floor. I don't know whether this is a general rule, but it certainly applies to Dima Volchenkov. Thank you for an open ear whenever I knocked on your door.

A special thanks goes to Hanne Litschewsky, our great secretary in E5. Due to Hanne, I could experience how comfortable, how helpful, yet sometimes essential it is to be supported in all the bureaucratic aspects of science.

All of this would have been a lot more difficult without the unconditional support of my family. I am very thankful to my parents, my parents-in-law, and especially to my wife Nannette.

Financial support of the German Federal Ministry of Education and Research (BMBF) through the project Linguistic Networks is also gratefully acknowledged (http://project.linguistic-networks.net). 



\section{Abstract}

This thesis introduces a Markov chain approach that allows a rigorous analysis of a class of agent-based models (ABMs). It provides a general framework of aggregation in agent-based and related computational models by making use of Markov chain aggregation and lumpability theory in order to link between the micro and the macro level of observation.

The starting point is a microscopic Markov chain description of the dynamical process in complete correspondence with the dynamical behavior of the agent model, which is obtained by considering the set of all possible agent configurations as the state space of a huge Markov chain. This is referred to as micro chain, and an explicit formal representation including microscopic transition rates can be derived for a class of models by using the random mapping representation of a Markov process. The explicit micro formulation enables the application of the theory of Markov chain aggregation - namely, lumpability - in order to reduce the state space of the micro chain and relate microscopic descriptions to a macroscopic formulation of interest. Well-known conditions for lumpability make it possible to establish the cases where the macro model is still Markov, and in this case we obtain a complete picture of the dynamics including the transient stage, the most interesting phase in applications.

For such a purpose a crucial role is played by the type of probability distribution used to implement the stochastic part of the model which defines the updating rule and governs the dynamics. Namely, if we decide to remain at a Markovian level, then the partition, or equivalently, the collective variables used to build the macro model must be compatible with the symmetries of the probability distribution $\omega$. This underlines the theoretical importance of homogeneous or complete mixing in the analysis of $»$ voterlike « models at use in population genetics, evolutionary game theory and social dynamics. On the other hand, if a favored level of observation is not compatible with the symmetries in $\omega$, a certain amount of memory is introduced by the transition from the micro level to such a macro description, and this is the fingerprint of emergence in ABMs. The resulting divergence from Markovianity can be quantified using information-theoretic measures and the thesis presents a scenario in which these measures can be explicitly computed.

Two simple models are used to illustrate these theoretical ideas: the voter model (VM) and an extension of it called contrarian voter model (CVM). Using these examples, the thesis shows that Markov chain theory allows for a rather precise understanding of the model dynamics in case of $\gg$ simple« population structures where a tractable macro chain can be derived. Constraining the system by interaction networks with a strong local structure leads to the emergence of meta-stable states in the transient of the model. Constraints on the interaction behavior such as bounded confidence 
or assortative mating lead to the emergence of new absorbing states in the associated macro chain and are related to stable patterns of polarization (stable co-existence of different opinions or species). Constraints and heterogeneities in the microscopic system and complex social interactions are the basic characteristics of ABMs, and the Markov chain approach to link the micro chain to a macro level description (and likewise the failure of a Markovian link) highlights the crucial role played by those ingredients in the generation of complex macroscopic outcomes. 


\section{CONTENTS}

1 INTRODUCTION 1

1.1 Agent-Based Models . . . . . . . . . . . . . . . . . 2

1.2 Markov Chain Description of Agent-Based Models . . . . . . 4

1.3 Markov Chain Aggregation .............. 5

1.4 Micro-Macro Transition in the Voter Model . . . . . . . . . 7

1.5 Thesis Structure . . . . . . . . . . . . . . . . . . 8

2 BACKGROUND 11

2.1 Agent-Based and Related Models . . . . . . . . . . . . . . 11

2.2 Basic Ingredients of Agent-Based Models . . . . . . . . . . . . 14

2.2.1 Agents as Elementary Units . . . . . . . . . . . . 15

2.2 .2 The Environment . . . . . . . . . . . . . . 16

2.2 .3 Interaction Rules . . . . . . . . . . . . . . . . 17

2.2 .4 Iteration Process . . . . . . . . . . . . . . 18

2.3 Markov Chain Formalization of Agent-Based Models . . . . . 19

2.4 Lumpability and State Space Aggregation . . . . . . . . . 21

2.4.1 Strong Lumpability . . . . . . . . . . . . . . . . 21

2.4.2 Weak Lumpability . . . . . . . . . . . . . . . 23

2.4.3 Nearly Lumpable and Non-Lumpable Aggregation . . 24

2.4.4 Time-Series-Based Aggregation . . . . . . . . . . 25

2.4.5 Aggregation in Dynamical Systems . . . . . . . . . 26

2.5 Motivation: Towards a Markov Chain Theory of Aggregation for Agent-Based Models . . . . . . . . . . . . . . . . 27

2.5.1 Bridging a Gap . . . . . . . . . . . . . . . . . 27

2.5.2 The Micro-Macro Link . . . . . . . . . . . . . . . 28

2.5.3 Computational Emergence and Aggregativity . . . . . 29

3 Agent-Based Models as Markov Chains 31

3.1 The Micro Level . . . . . . . . . . . . . . . . . . . . 32

3.1.1 The Grammar of an ABM . . . . . . . . . . . . 32

3.1.2 From Functional Graphs to Markov Chains . . . . . . 34

3.1.3 Single-Step Dynamics . . . . . . . . . . . . . 35

3.2 Macrodynamics, Projected Systems and Observables . . . . 38

3.2.1 Micro and Macro in ABMs . . . . . . . . . . 38 
3.2.2 Observables, Partitions and Projected Systems . . . . 39

3.2.3 Lumpability and Symmetry . . . . . . . . . . . . . 40

3.3 Opinion Dynamics and Projected Systems . . . . . . . . . . 41

3.3.1 The Macro Dynamics of the Binary Voter Model . . . 42

3.3.2 Transient Macro Dynamics . . . . . . . . . . . . 45

3.3.3 Exit Probabilities . . . . . . . . . . . . . . . . 52

3.3.4 Macrodynamics of the General Voter Model . . . . . . 52

3.3.5 Further Reduction . . . . . . . . . . . . . . . . 54

3.4 Bounded Confidence and the Emergence of Opinion Polarization 55

3.4 .1 The Unconstrained Case . . . . . . . . . . . . . 56

3.4.2 Bounded Confidence . . . . . . . . . . . . . . 57

3.4.3 Non-Lumpability for Further Reduction . . . . . . . . 59

3.4.4 Transient Behavior with Bounded Confidence . . . . . 60

3.5 Simple Generalizations . . . . . . . . . . . . . . . . . . . 64

3.6 Discussion . . . . . . . . . . . . . . . . . . . . . 65

4 From Network Symmetries to Markov Projections 67

4.1 Interaction Heterogeneity and Projection Refinement . . . . . 68

4.2 Social Structure at the Micro Level . . . . . . . . . . . . 70

4.3 Markovian Aggregation . . . . . . . . . . . . . . 72

4.3.1 Macroscopic Equivalence . . . . . . . . . . . . . 72

4.3.2 The Voter Model . . . . . . . . . . . . . . . . . 72

4.3.3 Single-Step Dynamics . . . . . . . . . . . . . . 73

4.4 The Two-Community Model . . . . . . . . . . . . . . . 74

4.4 .1 Model . . . . . . . . . . . . . . . . . . . . 74

4.4 .2 Markov Projection . . . . . . . . . . . . . 75

4.4 .3 Convergence Times . . . . . . . . . . . . . . . . 76

4.4.4 Quasi-Stationary Distribution . . . . . . . . . . . 79

4.5 On the Role of Peer-to-Peer Communication in an Opinion

Model with Leader . . . . . . . . . . . . . . . . . . 80

4.5 .1 Model . . . . . . . . . . . . . . . . . . . . 80

4.5.2 Markov Projection . . . . . . . . . . . . . . . 82

4.5.3 Simple Observations . . . . . . . . . . . . . . . 83

4.5.4 Influence of the Leader . . . . . . . . . . . . . . . . 83

4.5.5 Convergence Times . . . . . . . . . . . . . . . . . 84

4.5.6 Transient Behavior . . . . . . . . . . . . . . . 86

4.5.7 Alternative Interpretation . . . . . . . . . . . . . 88

4.6 The Ring . . . . . . . . . . . . . . . . . . . . . . . . . . . . . 88

4.7 Discussion . . . . . . . . . . . . . . . . . . . . . . . . . . . . . 89

5 Network Effects in the Contrarian Voter Model 91

5.1 The Contrarian Voter Model . . . . . . . . . . . . . . . . 91

5.1 .1 Model . . . . . . . . . . . . . . . . 92

5.1 .2 Micro Dynamics . . . . . . . . . . . . . . . . 92 
5.2 Homogeneous Mixing and the Two-Community Graph . . . . 94

5.2.1 Homogeneous Mixing . . . . . . . . . . . . . . 94

5.2.2 Stationary Dynamics for Homogeneous Mixing . . . . 96

5.2.3 Rate of Majority-Minority Switching . . . . . . . . . . 98

5.2 .4 Two-Community Model . . . . . . . . . . . . . 100

5.2.5 Stationary Dynamics on the Two-Community Graph . 102

5.3 Network Dynamics from the Macro Perspective . . . . . . . 105

5.3.1 Full Aggregation . . . . . . . . . . . . . . . 105

5.3.2 Network Influence on the Stationary Dynamics . . . . 107

5.3.3 The Two-Community Case . . . . . . . . . . . 108

5.4 The Two-Community Model as an Analytical Framework . . . 109

5.4.1 From Micro to Meso, and from Meso to Macro . . . . 109

5.4 .2 Why Lumpability Fails . . . . . . . . . . . . . . . . 111

5.4.3 Stationarity and Aggregation . . . . . . . . . . 112

5.4.4 Why Weak Lumpability Fails . . . . . . . . . . . . . 114

5.4.5 Measuring (Non)-Markovianity . . . . . . . . . . 117

5.5 Discussion . . . . . . . . . . . . . . . . . . . . 122

6 Aggregation and Emergence: A Synthesis 125

6.1 The Computational View on Emergence . . . . . . . . . 125

6.2 Challenging Definitions of Weak Emergence . . . . . . . 127

6.2.1 Unavoidability of Simulations . . . . . . . . . . . 127

6.2.2 Computational Incompressibility . . . . . . . . . 128

6.3 From Computational Incompressibility to Non-Aggregativity . 131 


\section{Chapter I}

\section{INTRODUCTION}

I think that nowadays most people would confirm that the world we live in is a complex one. Not only the problems that we face at a global scale (such as climate change and financial crises) but also many of our very personal dayto-day decisions (such as choosing between a fresh organic apple from oversee and a local apple maintained in an energy-expensive cooling chamber) involve nowadays, if carefully considered, the evaluation of entanglements of global scope. There is a high level of uncertainty in the evaluation of the consequences of our actions owing to the fact that those entanglements are often not clearly evident. There is also a high degree of freedom in what concerns the number of options that are in principle at our disposal, but if we do not sufficiently understand the functioning of the system there is no way to choose among them.

The »new science of complex systems « is an attempt to better understand the behavior of systems that are composed of elementary units and structures of mutual dependencies (Wechselwirkungen) between those units. The fundamental idea is that complex patterns of higher-level organization emerge in a dynamical system of interacting individuals that participate in a self-organizing process. While no central control is assumed to direct this process, the global emergences that are generated by it may well have an effect on the individual dynamics. Complexity, in this dynamical context, relates to the fact that higher-level patterns and processes are not easily understood by considering the dynamical mechanisms at the lower level only.

Of course, the fact that the behavior of many real-world systems is not predictable in simple way from the behavior of the system's components has been acknowledged long ago. Likewise, the observation that systems from very different fields and at different scales share important principles of organization. But especially the last two decades have witnessed a tremendous increase in scientific activity trying to make visible the empirical fingerprints of complex behavior (such as power law distributions or long range correlations) on the one hand, and to extract the underlying mechanisms and causal relations at work in those systems in order to really understand the fundamental principles of self-organized complexity on the other. For its 
enormous range of application - from biology to sociology, from physics to linguistics - complexity has become one of the most promising concepts in modern science.

In all of this, computational tools have become very important. Several methodological innovations are in fact enabled only by the general availability of relatively powerful computers: from the retrieval of information, statistical regularities and patterns from large amounts of data to the simulative testing of different behavioral assumptions in models of biological, social or cultural evolution. In general, the use of computational instruments does not make mathematics dispensable, to the contrary, it rather calls for the development of sound mathematical foundations of these new methods. In data science, this relates to questions concerned with statistical significance, algorithmic complexity, information theory, among many others; for computational models, it is related to proper formal model specifications, to the development of mathematical theories for multi-level systems and analytical solution strategies.

This thesis is concerned with the latter problem area. It develops mathematical concepts for the formal treatment of a class of computational models. Namely, it formulates agent-based models - models in which a finite number of agents interact according to simple behavioral assumptions - as Markov chains and makes use of Markov chain theory to derive explicit statements about the possibility of linking a microscopic agent model to the dynamical processes at the level of macroscopic observables. The questions that this thesis aims to address are inherently dynamic ones: the focus is not on the structural properties of certain agent networks, but rather on the dynamical processes at the micro and the macro level that differently structured systems give rise to. A particular aspect in that is the role that microscopic heterogeneity and constraints in the agent behavior play in the generation of macroscopic complexity. In this way, it touches upon questions related to the micro-macro link in social simulation and to computational emergence in general. Moreover, the question of deriving macroscopic descriptions with a minimal loss of information also goes to the heart of statistical mechanics.

\subsection{Agent-Based Models}

Recent improvements in multidisciplinary methods and, particularly, the availability of powerful computational tools are giving researchers an ever greater opportunity to investigate societies in their complex nature. The adoption of a complex systems approach allows the modeling of macrosociological or economic structures from a bottom-up perspective - understood as resulting from the repeated local interaction of socio-economic agents - without disregarding the consequences of the structures themselves on individual behavior, emergence of interaction patterns and social welfare. 
Agent-based models (henceforth ABMs) are at the leading edge of this endeavor. ABMs are an attempt to understand how macroscopic regularities may emerge through processes of self-organization in systems of interacting agents. The main idea is to place a population of agents characterized by a set of attributes within a virtual environment and specify simple rules of how agents interact with each other and the environment. The interaction rules are usually based on simple behavioral assumptions with the aim to mimic the individual behavior of real actors in their local environment. While the system is modeled at the microscopic level, its explanatory scope is the macro level. In that, ABMs follow the tradition of methodological individualism which claims $\gg$ that social phenomena must be explained by showing how they result from individual actions « (Heath, 2011, par. 1).

Agent-based systems are dynamical systems. Typically implemented on a computer, the time evolution is computed as an iterative process - an algorithm - in which agents are updated according to the specified rules. ABMs usually also involve a certain amount of stochasticity, because the agent choice and sometimes also the choice among different behavioral options is random. This is why Markov chain theory is such a good candidate for the mathematical formalization of ABMs.

The Voter Model (VM from now on) is a simple paradigmatic example (Kimura and Weiss, 1964; Castellano et al., 2009, among many others). In the VM, agents can adopt two different states, which we may denote as white $\square$ and black $\square$. The attribute could account for the opinion of an agent regarding a certain issue, its approval or disapproval regarding certain attitudes. In an economic context $\square$ and $\square$ could encode two different behavioral strategies, or, in a biological context, the occurrence of mutants in a population of individuals. The iteration process implemented by the VM is very simple. At each time step, an agent $i$ is chosen at random along with one of its neighboring agents $j$ and one of them imitates the state of the other. In the long run, the model leads to a configuration in which all agents have adopted the same state (either $\square$ or $\mathbf{\square}$ ). In the context biological evolution, this has been related to the fixation or extinction of a mutant in a population. The VM has also been interpreted as a simplistic form of a social influence process by which a shared convention is established in the entire population.

Let us consider an example simulation run of the VM to provide an intuition about its behavior (Fig. 1.1). Assume there are 20 agents connected by a chain such that an agent at position $i$ is connected to agents $i-1$ and $i+1$ (except the first and the last agent who have only one neighbor). Let the random initial population be $\mathbf{x}=(\mathbf{\square} \square \square \square \square \square \square \square \square \square \square \square \square \square \square(\mathbf{D})$ corresponding to the left-most column in Fig. 1.1. The time evolution is shown from left to right, the columns represent the configuration of the population each time after $10 \mathrm{VM}$ steps have been performed. This example shows two main features of the VM: (i.) the emergence of a meta-stable 


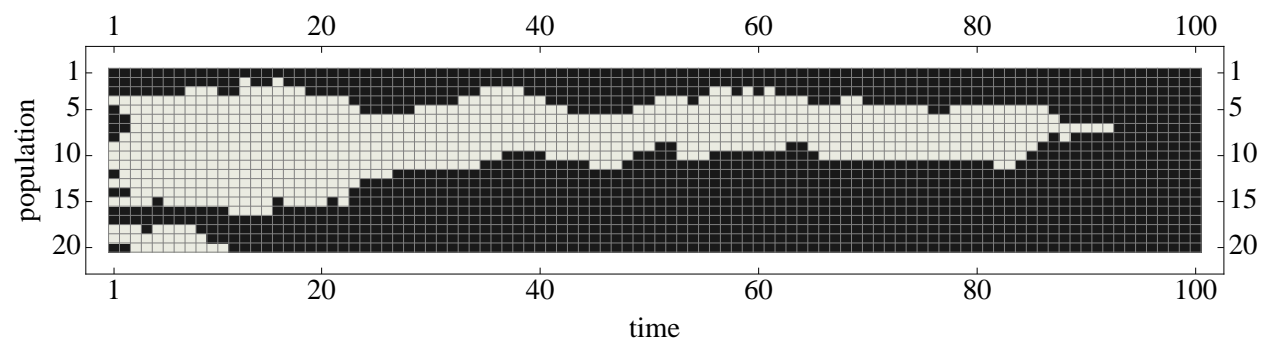

Figure 1.1: Example of the time evolution of the VM on the chain network.

transient state of local alignment, and (ii.) the final convergence to complete consensus. The first feature is clearly due to the interaction topology because initial local divergences are leveled with a high probability and once an areal of local alignment is achieved change is admitted, due to the chain topology, only at the borders of that domain. The second feature is a more general feature of the finite VM, sooner or later consensus occurs in every topology.

When designing an agent model, one is inevitably faced with the problem of finding an acceptable compromise between realism and simplicity. If many aspects are included into the agent description, the model might be plausible with regard to the individual behaviors, but it will be impossible to derive rigorous analytical results. In fact, it can even be very hard to perform systematic computations to understand the model dynamics if many parameters and rules are included. On the other hand, models that allow for an analytical treatment often oversimplify the problem at hand. The VM is good example of this kind. In ABM, we can find the whole spectrum between these two extremes. While simplicity is often favored by physicists in order to be able to apply their well-developed tools from statistical physics, more realistic descriptions are often desired by researchers in the humanities because they are interested in incorporating into the model a reasonable part of their qualitative knowledge at the micro and macro scales. Both views have, of course, their own merits.

\subsection{Markov Chain Description of Agent-Based Models}

This thesis is a contribution to interweaving two lines of research that have developed in almost separate ways: ABMs and Markov chains. The former represents the simplest form of a stochastic process while the latter puts a strong emphasis on heterogeneity and social interactions. The main expected output of a Markov chain strategy applied to ABM is a better understanding of the relationship between microscopic and macroscopic dynamical properties. Moreover, we aim to contribute not only to the understanding of the asymptotic properties of $\mathrm{ABM}$ but also to the transient mechanisms that 
rule the system on intermediate time scales. For practical purposes this is the most relevant information for two reasons: first, in many cases the chains are absorbing, so the asymptotic dynamics is trivial and second, they describe the evolution of the system before external perturbations take place and possibly throw it into a new setting.

The possibility of using Markov chains in the analysis of ABMs has been pointed out in Izquierdo et al. (2009). The main idea is to consider all possible configurations of the agent system as the state space of a huge Markov chain. While Izquierdo et al. (2009) mainly rely on numerical computations to estimate the stochastic transition matrices of the models, here we show for a class of models how to derive explicitly the transition probabilities $\hat{P}$ in terms of the update function $\mathbf{u}$ and a probability distribution $\omega$ accounting for the stochastic parts in the model. It turns out that ABMs with a sequential update scheme can be conceived as random walks on regular graphs.

Consider an ABM defined by a set $\mathbf{N}$ of agents, each one characterized by individual attributes that are taken from a finite list of possibilities. We denote the set of possible attributes by $\mathbf{S}$ and we call the configuration space $\boldsymbol{\Sigma}$ the set of all possible combinations of attributes of the agents, i.e. $\boldsymbol{\Sigma}=$ $\mathbf{S}^{N}$. Therefore, we denote an agent configuration as $\mathbf{x} \in \boldsymbol{\Sigma}$ and write $\mathbf{x}=$ $\left(x_{1}, \ldots, x_{i}, \ldots, x_{N}\right)$ with $x_{i} \in \mathbf{S}$. The updating process of the attributes of the agents at each time step typically consists of two parts. First, a random choice of a subset of agents is made according to some probability distribution $\omega$. Then the attributes of the agents are updated according to a rule $\mathbf{u}$, which depends on the subset of agents selected at this time. With this specification, ABMs can be represented by a so-called random map representation which may be taken as an equivalent definition of a Markov chain (Levin et al. (2009)). We refer to the process $(\boldsymbol{\Sigma}, \hat{P})$ as micro chain.

\subsection{Markov Chain Aggregation}

When performing simulations of an ABM we are actually not interested in all the dynamical details but rather in the behavior of certain macro-level properties that inform us about the global state of the system (such as average opinion, number of communities, etc.). The explicit formulation of ABMs as Markov chains enables the development of a mathematical framework to link a micro chain corresponding to an ABM to such a macro-level description of interest. Namely, from the Markov chain perspective, the transition from the micro to the macro level is a projection of the micro chain with state space $\boldsymbol{\Sigma}$ onto a new state space $\mathbf{X}$ by means of a (projection) map $\Pi$ from $\boldsymbol{\Sigma}$ to $\mathbf{X}$. The meaning of the projection $\Pi$ is to lump sets of micro configurations in $\boldsymbol{\Sigma}$ into an aggregate set according to the macro property of interest. Such a situation naturally arises if the ABM is observed not at the micro level of $\boldsymbol{\Sigma}$, but rather in terms of a measure $\phi$ on $\boldsymbol{\Sigma}$ by which all configuration in 
$\boldsymbol{\Sigma}$ that give rise to the same measurement are mapped into the same macro state, say $X_{k} \in \mathbf{X}$. An illustration of such a projection is provided in Fig. 1.2 .

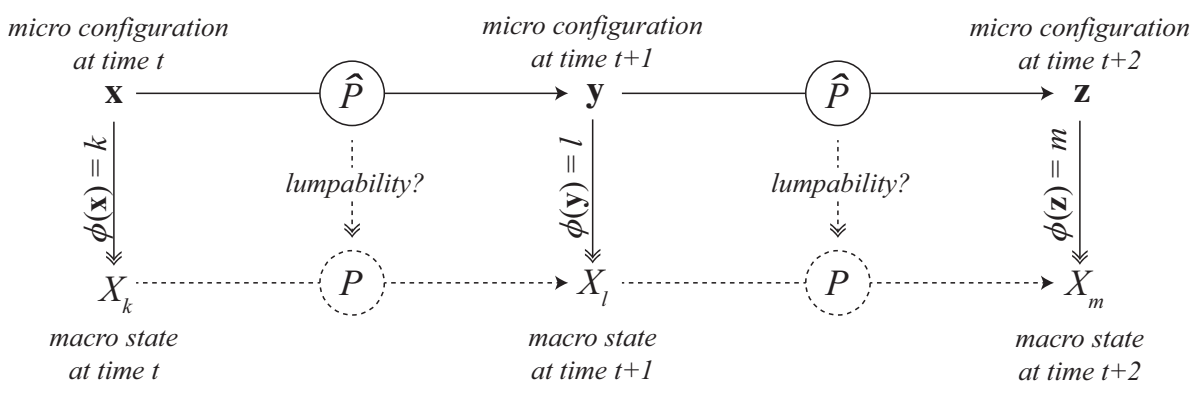

Figure 1.2: A micro process $(\mathbf{x}, \mathbf{y}, \mathbf{z} \in \mathbf{\Sigma})$ is observed $(\phi)$ at a higher level and this observation defines another macro level process $\left(X_{k}, X_{l}, X_{m} \in \mathbf{X}\right)$. The micro process is a Markov chain with transition matrix $\hat{P}$. The macro process is a Markov chain (with $P$ ) only in the case of lumpability.

There are two things that may happen when projecting a micro process onto a macroscopic state space $\mathbf{X}$. First, under certain conditions the macrolevel process is still a Markov chain. This case is known as lumpability in Markov chain theory and necessary and sufficient conditions are provided in a well-known textbook on finite Markov chains by Kemeny and Snell (1976). The question this thesis aims to address concerns, first of all, the conditions on the microscopic system and the projection construction that have to be met in order to lead to a macro process that is still a Markov chain. In this regard, if we decide to remain at a Markovian level, then the projection, or equivalently the collective variables to be used to build the macro model must be compatible with the symmetry of the probability distribution $\omega$. In turn, in the absence of any symmetry, there is no other choice than to stay at the micro-level because no Markovian macro-level description is possible in this case.

Secondly, and more generally, the price to pay in passing from the micro to the macro dynamics by such a projection construction is that the projected system is no longer a Markov chain. Long memory (even infinite) may appear in the projected system. Consequently, this setting can provide a suitable framework to understand how aggregation may lead to the emergence of long range memory effects. This opens up a series of interesting questions: for instance, why and in what sense does the behavior of the macro process deviate from Markovianity? How can we measure these deviations? Do we introduce memory or long-range correlations at the macro level by the very way we observe a process and is the emergence of these effects just due to an aggregation which is insensitive to microscopic heterogeneities? In particular, there is usually a strong interest in the effects that different 
interaction topologies have on the transient model dynamics as well as on the emergence of characteristic meta-stable situations, such as the persistent pattern of local alignment shown in Fig. 1.1. In that regard, how good does the mean field solution approximate network dynamics and for which networks does it provide acceptable approximations? Is there an alternative macro-level formulation that leads to better results? If yes, which properties can be captured by it? A micro-macro formalism may shed new light on some of these questions.

To my point of view, the non-Markovian case is in many ways even more interesting than the case of lumpability. In particular, because it relates microscopic heterogeneity to macroscopic complexity (structure generation). Constraints, heterogeneities in the microscopic system and complex social interactions are the basic characteristics of ABMs, and the Markov chain approach to link the micro chain to a macro level description (and likewise the failure of a Markovian link) highlights the crucial role played by those ingredients in the generation of complex macroscopic outcomes. The formalization of the relations between the micro and the macro levels in the description of the dynamics of ABMs as well as their mathematical characterization is a step towards a mathematical theory of emergence in complex adaptive systems.

\subsection{Micro-Macro Transition in the Voter Model}

Let us exemplify the link between a micro and a macro chain by Markov chain aggregation for the VM. From the microscopic perspective, the VM corresponds to an absorbing random walk on the $N$-dimensional hypercube. If $N$ agents can be in two different states, the set of all agent configurations $\boldsymbol{\Sigma}$ is the set of all bit-strings of length $N$. Due to the dyadic conception of the interaction along with a sequential update scheme only one agent may change at a time which means that transitions are only possible between configurations that differ in at most one bit. The structure of the VM micro chain is shown for a small system of three agents in the upper part of Fig. 1.3.

In the VM, the most typical level of observation is to count the number of agents in the different states. In hypercube terminology this corresponds to the Hamming weight (i.e., $\phi(\mathbf{x})=h(\mathbf{x}))$. By the projection that this observation induces, all micro configurations with the same number of (say) white agents are mapped into the same macro state. If $k$ is the number of white agents $(h(\mathbf{x})=k)$, we denote the respective macro state as $X_{k}$. Therefore, if we are dealing with a system of $N$ agents, there are $N+1$ macro states which is a tremendous reduction compared to the $2^{N}$ micro configurations. The projection construction for the VM is shown in Fig. 1.3.

Voter-like models - as used in physics-inspired models of social dynamics as well as in population genetics or evolutionary dynamics - are nice examples 


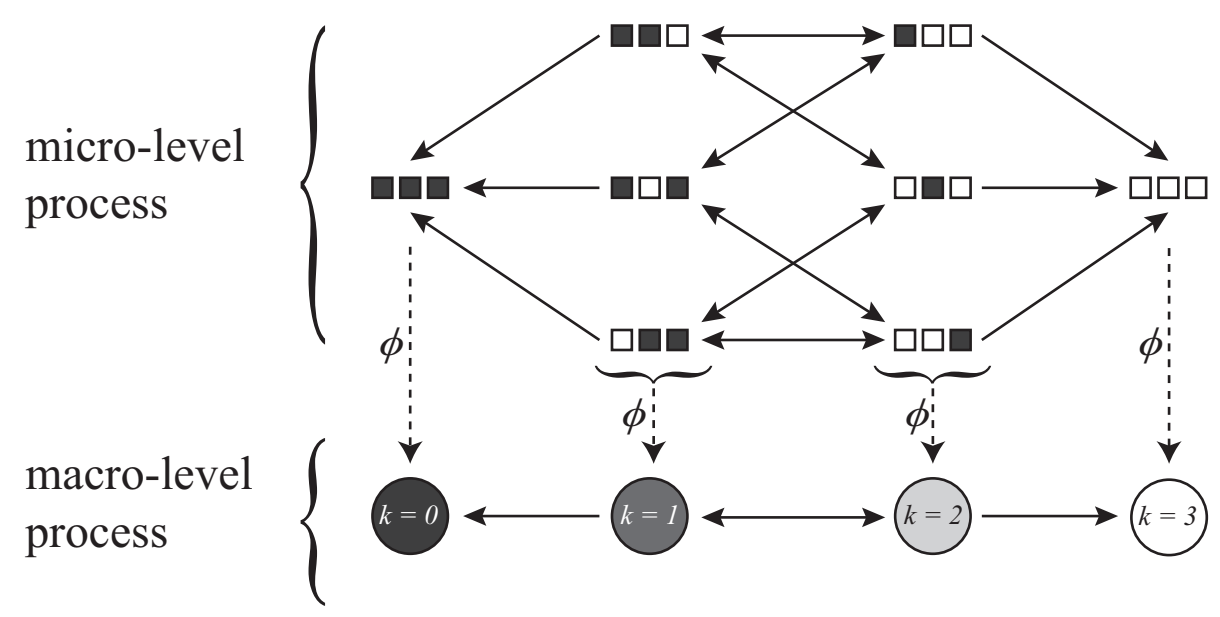

Figure 1.3: Micro and macro level in the VM with three agents.

where such a projection construction is particularly meaningful. Namely, because it corresponds to the most typical description of the model dynamics in terms of attribute frequencies. Lumpability allows to determine conditions for which the macro chain on $\mathbf{X}=\left(X_{0}, \ldots, X_{k}, \ldots, X_{N}\right)$ is again a Markov chain and, as will be shown in Chapter 3, this requires that the probability distribution $\omega$ over agent choices must be invariant under the group $\mathcal{S}_{N}$ of all the permutations of $N$ agents, and therefore uniform. This underlines the theoretical importance of homogeneous mixing and respectively the complete graph in the analysis of the VM and related models.

\subsection{Thesis Structure}

The thesis is organized into seven chapters. Chapter 2 provides an elementary introduction to ABMs and discusses different ideas for their mathematical formalization. It proceeds with a review of different approaches to lumpability in Markov chains and motivates their application to ABMs.

Chapter 3 develops the most important theoretical ideas and applies them to the VM with homogeneous mixing. The first part of Chapter 3, addresses the micro level and shows that a class of ABMs are Markov chains on regular graphs. This is followed by general description of the transition from the micro to the macro level. After that, a detailed analysis of the VM with homogeneous mixing is presented including the multi-state version of the model. Chapter 3 also shows that interaction constraints such as bounded confidence may lead to the stable co-existence of polarization. The final part is devoted to a discussion of most important implications.

In the next chapter, Chapter 4, we discuss what happens in the case 
of inhomogeneous interaction probabilities. A systematic approach to aggregation is developed which exploits all the dynamical redundancies that have its source in the agent network on which the model is implemented. This enables the analytical treatment of a leader-follower system as well as the two-community model in which two strongly connected groups interact weakly with one another. Implications are discussed at the end.

The main objective of Chapter 5 is to study the influence of interaction topology on the macro-level dynamics in the case of non-lumpability. The contrarian voter model (CVM) is used here. We start from the micro-level description and derive a macro chain for homogeneous mixing as well as a meso-level description for the two-community graph. This provides an analytical scenario to study the discrepancy between the homogeneous mixing case and the model on a slightly more complex topology. It shows that memory effects are introduced at the macro level when we aggregate over agent attributes without sensitivity to the microscopic details and quantifies these effect using concepts from information theory. The possibility of weak lumpability is also discussed.

While the specific issues are discussed at the end of each chapter, Chapter 6 aims at a synthetic view on how this work may contribute more generally to the study of complexity and emergence. A definition of emergence in terms of lumpability provides a link between two different perspectives on emergence, namely, the concept of dynamical incompressibility and Wimsatt's notion of non-aggregativity.

Finally, Chapter 7 draws a conclusion on the project as a whole and outlines some ideas and challenges for future research. 


\section{BACKGROUND}

This thesis is a contribution to interweaving two lines of research that have developed in almost separate ways: Markov chains and agent-based models (ABMs). The former represents the simplest form of a stochastic process while the latter puts a strong emphasis on heterogeneity and social interactions. This chapter provides an introduction to ABMs and reviews approaches to use Markov chains in their analysis.

The main expected output of the Markov chain strategy applied to ABMs is a better understanding of the relationship between microscopic and macroscopic dynamical properties. This brings into the discussion concepts of aggregation and emergence, and it also relates to macroscopic mean-field formulations as a substantial tool in the statistical mechanics approach to social dynamics. A complete review of the literature dealing with these topics is clearly beyond the scope of this chapter which is rather aimed at introducing the most important concepts with reference to ABMs and Markov chains. Especially the physics-inspired approach to social dynamics has attracted a lot of interest in the last years and a huge number of papers is still produced every year. For a relatively coherent review (though, may be, no longer completely up-to-date), the reader may be referred to Castellano et al. (2009).

\subsection{Agent-Based and Related Models}

ABMs are an attempt to understand how macroscopic regularities may emerge through processes of self-organization in systems of interacting agents. A system at question is modeled at the microscopic level by specifying the elementary units of that system - the agents - and implementing simple rules for how these agents interact with one another. Typically implemented on a computer, the time evolution of such a system is computed as an iterative process - an algorithm - in which agents are updated according to the specified rules. One of the main purposes of this modeling strategy is $\gg$ to enrich our understanding of fundamental processes « (Axelrod, 1997, 25) underly- 
ing certain observed patterns, or to »explore the simplest set of behavioral assumptions required to generate a macro pattern of explanatory interest « (Macy and Willer, 2002, 146).

One paradigmatic example of ABMs is Reynolds model of the flocking behavior of birds (Reynolds, 1987). While the modeling of a flock as such is difficult, quite realistic flocking behavior is achieved if the individual birds follow simple rules of how to react upon the action of other individuals in their neighborhood. Another well-known example is Schelling's model of segregation (Schelling, 1971). Here, two kinds of householders (say black and white) located on a lattice are endowed with a slight preference to settle in a neighborhood with more households of the same kind. Running that system leads to a clear spatial segregation at the global level even if the homophily preference is small. Similar effects can be observed in models of opinion and cultural dynamics, see, for instance, Axelrod (1997); Deffuant et al. (2001); Hegselmann and Krause (2002); Banisch et al. (2010). Another paradigmatic problem that has been addressed by ABM research is the emergence of a set of norms or common conventions. In the naming game proposed by Steels (1997), for instance, robots learn common word-object relations in a communication process based on trail and error. Other models in which an initial plurality in a population of agents evolves to a common consensus state include various models of opinion formation with the VM as the most simple representative (see Castellano et al., 2009 for a review of these models).

It is common to trace back the history of ABMs to the cellular automata (henceforth CA) designed by von Neumann and Ulam (von Neumann, 1951) and later shaped by Conway (Berlekamp et al., 1982) and Wolfram (1983, 2002). And in fact, many ABMs can be viewed as a stochastic CA with asynchronous update. The methods developed in this work apply precisely to that type of models.

However, even some years before von Neumann and Ulam came up with the first CA design, another type of »individual-based « model had been introduced in a branch of theoretical biology which is today called population genetics (see Li, 1977 for a collection of the seminal papers in that field). Wright and Fisher (along with Haldane known as the founders of population genetics) advocated a simple model for the evolution of allele frequencies (Wright, 1932) based on microscopic assumptions of gene transmission from the parent to the children generation. In 1958, Moran (1958) made use of Markov chain theory to study a modified model and introduced what today is known as the Moran process. Later, Kimura went further in this line of research on a neutral theory of evolution with the stepping stone model Kimura and Weiss (1964) which still later became known as the voter model (abbreviated by VM throughout this thesis). From the very beginning population genetics developed as a mathematical discipline and has inspired various solution strategies from probabilistic methods including Markov chains and coalescing random walks to mean-field approaches in statistical physics. 
The biological literature on evolutionary dynamics on graphs has mainly started from the model proposed by Moran (1958). In the Moran model, at each time step, an individual is chosen at random to reproduce and replaces a second one chosen at random as well. In the original model, there is no population structure which means that all individuals are chosen with equal probability. Therefore - this is something that will be made explicit in the third chapter of this thesis - the dynamics can be formulated as a birth-death random walk on the line. See Claussen and Traulsen (2005); Traulsen et al. (2005); Nowak (2006) for treatments of the associated Moran process. While early studies Maruyama (1974); Slatkin (1981) had indicated that population structure has no or only little effect on the model behavior, it has recently been shown that population structure can have a significant influence (Liberman et al., 2005; Nowak, 2006; Shakarian et al., 2012; Voorhees and Murray, 2013; Voorhees, 2013, among many others). The setting - sometimes referred to as evolutionary graph theory (Liberman et al., 2005) - is usually as follows: suppose the is a population of $N$ individuals with fitness 1 ; suppose that a mutant with fitness $r$ is introduced in one of the individuals; what is the probability that the mutant invades the entire population? The Moran case of unstructured populations is usually taken as a benchmark such that a graph which leads to a fixation probability different from the unstructured case are said to suppress or respectively enhance selection.

In the physics literature, the analysis of binary models as the VM is usually based on mean-field arguments. The system dynamics is traced in form of an aggregate order parameter and the system is reformulated on the macro-scale as a differential equation which describes the temporal evolution of that parameter. In many cases, the average opinion (due to the analogy to spin systems often called »magnetization «) has proven to be an adequate choice, but sometimes the number of (re)active interfaces yields a more handable transformation (e.g., Frachebourg and Krapivsky, 1996; Krapivsky and Redner, 2003; Vazquez and Eguíluz, 2008). A mean-field analysis for the VM on the complete graph was presented by Slanina and Lavicka (2003), and naturally, we come across the same results using our method (Sec. 3.3.2). Slanina and Lavicka (2003) derive expressions for the asymptotic exit probabilities and the mean time needed to converge, but the partial differential equations that describe the full probability distribution for the time to reach the stationary state is too difficult to be solved analytically (Slanina and Lavicka, 2003, 4). Further analytical results have been obtained for the VM on $d$-dimensional lattices (Cox, 1989; Frachebourg and Krapivsky, 1996; Liggett, 1999; Krapivsky and Redner, 2003) as well as for networks with uncorrelated degree distributions (Sood and Redner, 2005; Vazquez and Eguíluz, 2008). It is noteworthy, that the analysis of the VM (and more generally, of binary-state dynamics) on networks has inspired a series of solution techniques such as refined mean-field descriptions (e.g., Sood and Redner, 2005; Moretti et al., 2012), pairwise approximation (e.g., 
De Oliveira et al., 1993; Vazquez and Eguíluz, 2008; Schweitzer and Behera, 2008; Pugliese and Castellano, 2009) and approximate master equations (e.g., Gleeson, 2011, 2013).

The early works in population genetics (Fisher, 1930, in particular) have inspired still another modeling approach that is related to ABMs, namely, evolutionary game theory (see Smith, 1982 for a seminal volume and Roca et al., 2009 for a recent review). Here, games are designed in which agents repeatedly play against one another adopting one out of a set of predefined strategies. A fitness is assigned to the combinations of strategies and the population evolves as a response to this fitness. As in the framework of statistical mechanics, the model evolution is typically captured in form of differential equation describing the evolution of the (relative) frequencies of the different strategies, referred to as replicator dynamics in this context (Taylor and Jonker, 1978; Schuster and Sigmund, 1983; Hofbauer and Sigmund, 2003). One of the main purposes of this work is to spell out explicitly how to link the dynamics at the micro level to these macroscopic descriptions.

Finally, it is worth mentioning that research in economics has experienced a growing interest in modeling economic phenomena as the result of the interactions of heterogeneous individuals (Tesfatsion and Judd, 2006). In particular in the field of finance, this has led to the development of ABMs for the identification of (macro) patterns of collective dynamics from (micro) investor heterogeneity in many financial settings (Cont and Bouchaud, 2000; LeBaron, 2000; Hommes, 2006; Preis et al., 2013). Noteworthy, there is also a number of empirical applications of Markov chains in the field of finance (e.g., Corcuera et al., 2005; Nielsen, 2005; Norberg, 2006). Interaction and heterogeneity on the one hand, and non-Gaussianity, heavy tails and long-range correlations on the other appear to be natural features of modern economies, to which the formerly dominating tradition of modeling representative agents has, to a large extent, paid little attention. This thesis shows that memory effects at the macroscopic level are an immediate consequence of microscopic heterogeneity and it may therefore contribute to the identification of the relevant microscopic mechanisms that presumably play a role in the market.

\subsection{Basic Ingredients of Agent-Based Models}

Roughly speaking, an ABM is a set of autonomous agents which interact according to relatively simple interactions rules with other agents and the environment. The agents themselves are characterized (or modeled) by a set of attributes some of which may change over time. Interaction rules specify the agent behavior with respect to other agents in the social environment and in some models there are also rules for the interaction with an external environment. Accordingly, the environment in an ABM is sometimes a model of 
a real physical space in which the agents move and interact upon encounter, in other models interaction relations between the agents are defined by an agent interaction network and the resulting neighborhood structure.

In the simulation of an $\mathrm{ABMs}$ the interaction process is iterated and the repeated application of the rules gives rise to the time evolution. There are different ways in which this update may be conceived and implemented. As virtually all ABMs are made to be simulated on a computer, I think it is reasonable to add to the classic three-fold characterization of ABMs as »agents plus interactions plus environment « a time-component because different modes of event scheduling can be of considerable importance.

\subsubsection{Agents as Elementary Units}

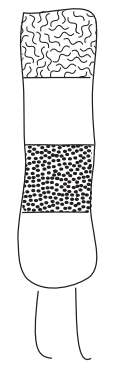

Figure 2.1: Caricature of an agent.

In this work, we deal with agents that are characterized by a finite set of attributes. The agent in the example above, for instance, can be described by a four-dimensional vector encoding the four different attributes from top to the bottom. In the sequel we will denote the state of an agent $i$ as $x_{i}$. Let us assume that, in this example, for each of the four features there are two alternatives: blank or covered. Then we could encode its state from the top to the bottom as $x_{i}=(\square \square \square)$, $\square$ accounting for »covered « and $\square$ for »blank «. It is clear that, in this case, there are $2^{4}=16$ possible agent states and we shall refer to this set as attribute space and denote it by $\mathbf{S}=\{\boldsymbol{\square}, \square\}^{4}$.

For the purposes of this work, the meaning of the content of such attributes is not important because the interpretation depends on the application for which the agent model is designed. It could account for the behavioral strategies with regard to four different dimensions of an agent's live, it could be words or utterances that the agent prefers in a communication with others, or represent a genetic disposition. Consequently, $x_{i}$ may encode static agent attributes or qualities that change in the life-time of the agent, or a mixture of static and dynamic features.

ABMs are usually an attempt to analyze the behavior of an entire population of agents as it follows from many individual decisions. Therefore, there is actually a number of $N$ agents each one characterized by a state 
$x_{j} \in \mathbf{S}$. We shall denote the configuration of $N$ agents by $\mathbf{x}=\left(x_{1}, \ldots, x_{N}\right)$ and call this an agent profile or agent configuration.

\subsubsection{The Environment}

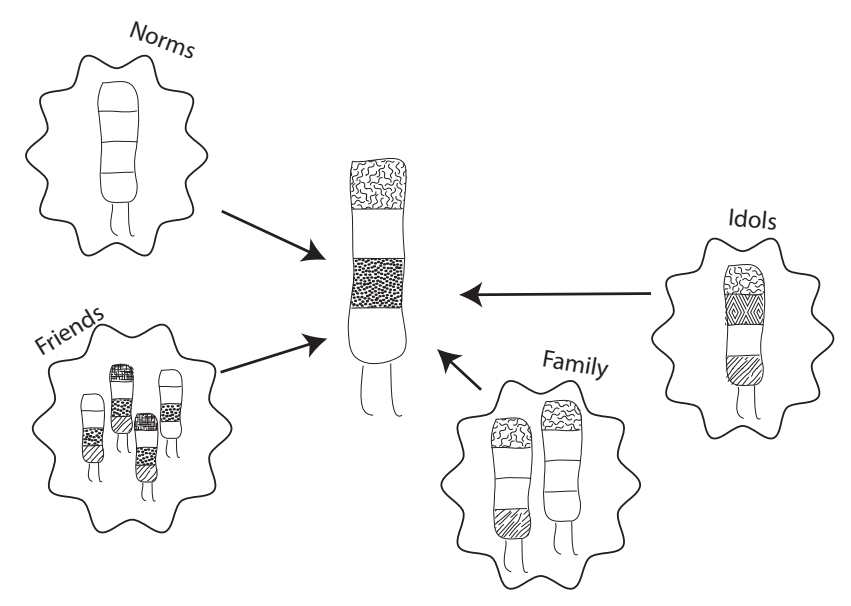

Figure 2.2: A social agent and its environment.

For the moment, we keep our eye on a single agent and consider environmental aspects an agent may take into account for its decisions. As noted earlier, the environment can be a model of real physical space in which the agent moves around according to some movement rules and where interaction with other individuals occurs whenever these agents encounter in the physical space. But environment is actually a more abstract concept in ABMs. It also accounts for the agent's social environment, its friends and family, as well as for social norms, idols or fads brought about by television. In a biological context the environment might be modeled by a fitness function which assigns different reproduction chances to different agent attributes $x_{i}$.

One of the most important aspects in ABMs is the introduction of social relations between the agents. Family structures and friendship relations are usually included by means of a graph $G=(\mathbf{N}, \mathbf{E})$, the so-called social network. Here $\mathbf{N}$ denotes the set of agents and $\mathbf{E}$ is the set of connections $(i, j)$ between the agents. These connections, called edges, can be weighted to account for the strength of the relation between agent $i$ and $j$ and negative values might even be taken to model adverse relations. Very often, the probability that two agents are part of the same interaction event depends directly on their connectivity in $G$. In fact, many models, especially simple physics-inspired models of social dynamics, take into account only a social interaction network and leave other environmental aspects out of consideration. 


\subsubsection{Interaction Rules}

In an interaction event, typically, an agent has to take a decision on the basis of the information within its environment. This includes a set of other agents, friends, family, with which the agent is connected as well as global information about norms, and possibly, internalized individual preferences. Each decision corresponds to an update of the agent's state $x_{i} \rightarrow y_{i}$ where we use $x_{i}$ to denote the agent state before the interaction takes place and $y_{i}$ to denote the updated state.

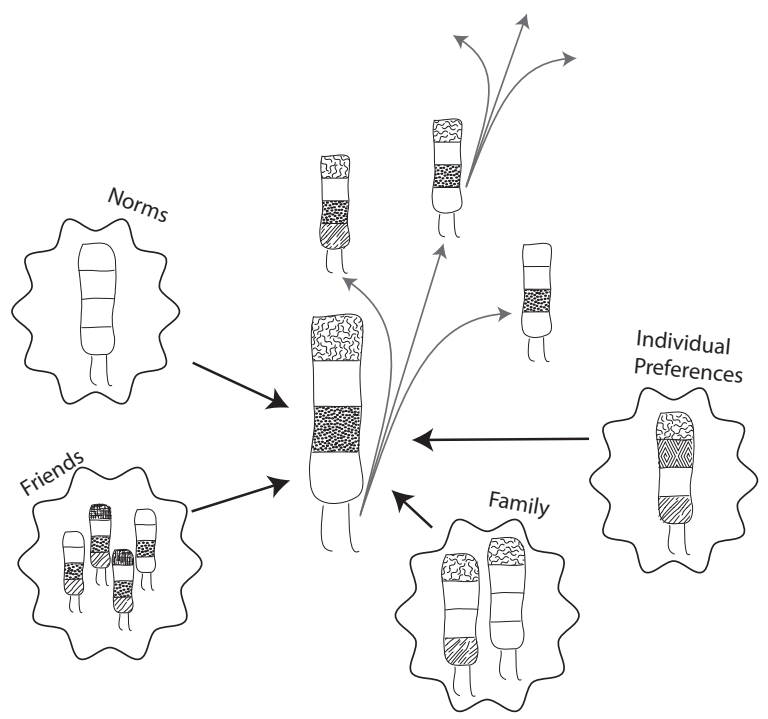

Figure 2.3: Interaction and iteration involve indeterminism and stochasticity. Therefore, there are several possible future states to which an agent may evolve in one step.

Usually, an agent in a specific situation has several well-defined behavioral options. Although in some sophisticated models agents are endowed with the capacity of evaluating the efficiency of these options, it is an important mark of ABMs that this evaluation is based on incomplete information and not perfect, and therefore the choice an agent takes involves a level of uncertainty. That is, a probability is assigned to the different options and the choice is based on those probabilities. This means that an agent in state $x_{i}$ may end up after the interaction in different states $y_{i}, y_{i}^{\prime}, y_{i}^{\prime \prime}, \ldots$ The indeterminism introduced in this way is an essential difference to neoclassical game-theoretic models and rational choice theory. And it is the reason why Markov chain theory is such a good candidate for the mathematical formalization of ABMs. 


\subsubsection{Iteration Process}

The conceptual design of an ABM is mainly concerned with a proper definition of agents, their interaction rules and the environment in which they are situated. In order to study the time evolution of such a system of interdependent agents, however, it is also necessary to define how the system proceeds from one time step to the other. As virtually all ABMs are simulation models implemented on a computer, it is an inherent part of the modeling task to specify the order in which events take place during an update of the system.

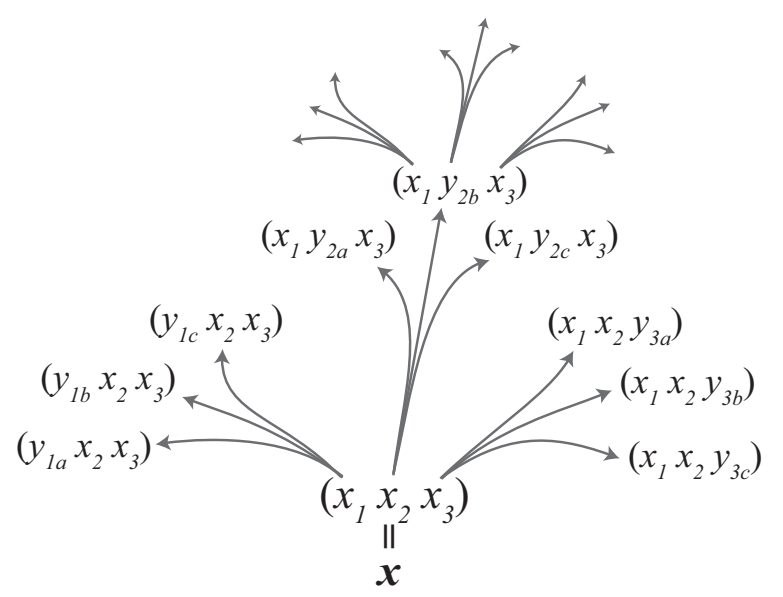

Figure 2.4: Possible paths in a small system of three agents (labeled by $1,2,3)$ where every agent has three alternative options (labeled by $a, b, c$ ).

A typical procedure is to first choose an agent at random (say agent $i$ ). The current agent state $x_{i}$ along with all the information this agent has about his environment defines the actual situation of the agent and determines the different behavioral options. If, in this situation, there is more than one option available to the agent, in a second step, one of these options has to be chosen with a certain probability. In this light, the update of an ABM can be seen as a stochastic choice out of a set of deterministic options, where stochastic elements are involved first into the agent choice and second into the selection of one out of several well-defined alternatives.

This procedure is illustrated for a small system of three agents in Fig. 2.4. The current agent profile is $\mathbf{x}=\left(x_{1} x_{2} x_{3}\right)$. To proceed to the next time step, first, one of the agents is chosen to update its state with some probability. So the new configuration of the system (denoted as $\mathbf{y}$ ) might differ from $\mathbf{x}$ in the first $\left(x_{1} \rightarrow y_{1}\right)$, the second $\left(x_{2} \rightarrow y_{2}\right)$, or the third $\left(x_{3} \rightarrow y_{3}\right)$ position. As every agent himself has three different behavioral alternatives chosen with a certain probability (as in Fig. 2.3), there are three paths for each potential agent $\left(x_{1} \rightarrow y_{1 a}\right.$ or $x_{1} \rightarrow y_{1 b}$ or $\left.x_{1} \rightarrow y_{1 c}\right)$. As a whole, there are thus 9 $(=3 \times 3)$ possible future agent configurations $\mathbf{y}$ to which the update process 
may lead with a well-defined probability after a single step.

In the update scheme described above the agents are updated one after the other and therefore this scheme is called sequential or sometime asynchronous update. A single time step corresponds in this scheme to a single interaction event. An alternative update scheme is synchronous or simultaneous update where the agents are updated $\gg$ in parallel $\ll$. That is, given a system profile $\mathbf{x}$, all agents are chosen, determine and select their behavioral options at the same time. The transition structure becomes more complex in that case mainly because the number of possible future configurations $\mathbf{y}$ is large compared to the asynchronous case since all agents change at once and there are several paths for each agent. In our example system of three agents each with three different options, the number of possible future states $\mathbf{y}$ is $27\left(=3^{3}\right)$. Most ABMs, however, have been implemented using the sequential update scheme, may be because the sequential philosophy of traditional programming languages made it more convenient. In this work, we will also concentrate on the sequential scheme.

\subsection{Markov Chain Formalization of Agent-Based Models}

The ABM approach is first and foremost a computational methodology and the mathematical formalization of the models is in its infancy. This is probably due to the fact that a major motivation in the development of ABMs has been to relax a series of unrealistic assumptions made in other modeling frameworks just in order to keep mathematical tractability; namely, rationality, perfect information, agent homogeneity, and others. The other side of the coin is that the focus on computer models and algorithms makes difficult the comparison of different models and also complicates a rigorous analysis of the model behavior. In fact, the problems of code verification and model comparison including the discussion of standards for the replication of ABMs has nowadays become an area of research in its own (e.g., Axtell et al., 1996; Axelrod, 2003; Hales et al., 2003; David et al., 2005; Grimm et al., 2006; Wilensky and Rand, 2007; Galán et al., 2009). As a matter of fact, many of those problems would actually vanish with a sound mathematical formulation of an ABM. On the other hand, it is also clear that the precise mathematical specification of a high-dimensional system of heterogeneous interacting agents along with their update mechanisms can be cumbersome in more complex ABM.

To the authors knowledge, the first systematic approach to the development of mathematical formalism for ABMs in general is due to Laubenbacher and co-workers. Laubenbacher et al. (2009) review existing formal frameworks that have the potential to model ABMs, such as cellular automata and finite dynamical systems and argue for the latter as an appropriate 
mathematical framework to represent ABMs. However, the probabilistic nature of most ABMs can only be accounted for by the stochastic version - the so-called stochastic finite dynamical systems - the analysis of which $»$ is still in its infancy« (ibid., 14). On the other hand, Laubenbacher et al. (2009) recognize that stochastic finite dynamical systems give rise to Markov chains. However, for reasons that do not become very clear in their paper, the authors argue:

$»$ To understand the effect of structural components such as the topology of the dependency graph or the stochastic nature of the update, it is important to study them not as Markov chains but as SFDS [stochastic finite dynamical systems] « (ibid., 10)

I clearly disagree with them in this point, because the microscopic specification of ABMs as Markov chains developed in this thesis turns out to be a useful starting point for further analysis. But of course, the incentive of Laubenbacher et al. (2009) to further elaborate the theory of stochastic dynamical systems in order to derive rigorous results for ABMs in future is highly appreciable.

The usefulness of the Markov chain formalism in the analysis of ABMs has first been realized by Izquierdo et al. (2009). The authors look at 10 wellknown social simulation models and discuss for each of them how to represent the model as a time-homogeneous Markov chain. Among the ABMs studied in Izquierdo et al. (2009) are the Schelling segregation model (Schelling (1971), for which some analytical results are available, for example, in Pollicott and Weiss (2001); Grauwin et al. (2010)), the Axelrod model of cultural dissemination (Axelrod (1997), see also Castellano et al. (2000) for a mean-field approximation) and the sugarscape model from Epstein and Axtell (1996). Noteworthy, the sugarscape model - one of the reference models in the field of social simulation - contains virtually all features that may occur in ABMs: heterogeneous agents placed in a dynamic spatial environment, death and birth of agents, various static and dynamic attributes that may evolve on different time scales.

The main idea of Izquierdo et al. (2009) is to consider all possible configurations of the system as the state space of a huge Markov chain and the construction of that state space is actually the main challenge for Izquierdo and co-workers. Despite the fact that all the information of the dynamics on the ABM is encoded in a Markov chain, however, it is difficult to learn directly from this fact, due to the huge dimension of the configuration space and its corresponding Markov transition matrix. The analyses provided in Izquierdo et al. (2009) are essentially based on the classification of states into transient and absorbing communicating classes which allows some statements about the convergence as times goes to infinity.

The paper of Izquierdo et al. (2009) is designated $\gg$ for researchers who may not have a strong mathematical background « (par.1.1) and probably 
therefore lacks rigorous arguments sustaining some of the results. Most fundamentally, there is no proof that the process on the constructed configuration space indeed satisfies the Markov property. Their work also mainly relies on numerical computations to estimate the stochastic transition matrices of the models. Both issues are addressed in this thesis. The explicit computation of transition probabilities, in particular, allows for the application of the theory of Markov chain aggregation in order to reduce the state space of the model.

\subsection{Lumpability and State Space Aggregation}

The state space of a Markov chain derived by considering as states all possible system configurations is far too big to directly use the respective transition matrix for exact numerical computations. As an example, consider a model with binary agent attributes such as the VM. A system of $N$ agents will lead to a Markov chain of size $2^{N}$ which for our introductory example of only 20 agents (Fig. 1.1) leads to a chain with more than a million states. In order to use the Markov chain machinery for ABMs, the system size has to be reduced in some way.

\subsubsection{Strong Lumpability}

This brings lumpability into play as a way to combine and aggregate the states of a Markov chain so that the process at the aggregate level is still a Markov chain. Consider that the state space of a Markov chain is $\boldsymbol{\Sigma}$ and the transition probabilities between all pairs of states in $\boldsymbol{\Sigma}$ are given by the $|\boldsymbol{\Sigma}| \times|\boldsymbol{\Sigma}|$ transition matrix $\hat{P}$. Throughout this work, the chain $(\boldsymbol{\Sigma}, \hat{P})$ will be called micro chain and, respectively, the states in $\boldsymbol{\Sigma}$ micro states. Now assume that $\mathbf{X}=\left(X_{0}, X_{1}, \ldots, X_{n}\right)$ is a partition of $\boldsymbol{\Sigma}$ where each $X_{k}$ contains a set of micro states in $\boldsymbol{\Sigma}$, such the $X_{k}$ are disjoint $\left(X_{k} \cap X_{s}=\emptyset\right.$ for any pair of aggregate sets) and for the union of all sets $\bigcup_{i=0}^{n} X_{i}=\boldsymbol{\Sigma}$. Such a situation naturally arises if the process is observed not at the micro level of $\boldsymbol{\Sigma}$, but rather in terms of a measure on $\boldsymbol{\Sigma}, \phi: \boldsymbol{\Sigma} \rightarrow\{0,1, \ldots, n\}$, by which all states in $\boldsymbol{\Sigma}$ that give rise to the same measurement are mapped into the same aggregate set $X_{k}$ (also referred to as macro states). An important question that arises in such a setting is whether the new aggregate process on $\mathbf{X}$ is still a Markov chain or not. This is what lumpability is about. The lumpability theory adopted for the purposes of this thesis is largely based on Kemeny and Snell (1976), which is, to the authors knowledge, the first textbook in which the strong as well as the weak form of lumpability are discussed with some detail. Notice that there are some other early and seminal works on lumpability, such as Burke and Rosenblatt (1958); Rosenblatt (1959); Rogers and Pitman (1981). 
To illustrate the concept of strong lumpability, let us use the Land of Oz example repeatedly considered in Kemeny and Snell (1976) (see pages 29/30 for the introduction of the example and page 125 for the lumpability example). There, a three-state Markov chain is formed which approximates how the whether develops from one day to the other. There is rain $(R)$, nice whether $(N)$ and snow $(S)$ and the transition rates are given by

$$
\hat{P}=\begin{gathered}
R \\
N
\end{gathered}\left(\begin{array}{ccc}
1 / 2 & 1 / 4 & 1 / 4 \\
1 / 2 & 0 & 1 / 2 \\
1 / 4 & 1 / 4 & 1 / 2
\end{array}\right) .
$$

Therefore, a nice day is never followed by a nice day, but there is an equal chance to have rain or snow. For a rainy day as well as for a day with snow, on the contrary, there is a chance of $1 / 2$ that the whether remains as it is for the next day, and the remaining options are equally likely with probability $1 / 4$. From this assignment of probabilities, we can already see that the behavior for rain $(R)$ and snow $(S)$ is actually equal and therefore we may combine the two states into a $»$ macro « state called $\gg$ bad whether « $(B=\{R, S\})$. Hence, the states space is partitioned into two sets: $N$ on the one hand and $B=\{R, S\}$ on the other. Now, as the probability that nice whether follows is equal for $R$ and $S$ the transition matrix of the new chain is uniquely defined by:

$$
P=\stackrel{N}{B}\left(\begin{array}{cc}
0 & 1 \\
1 / 4 & 3 / 4
\end{array}\right)
$$

It is the equality of conjoint transition rates from the states that shall be combined to all the other partitions $(\hat{P}(R, N)=\hat{P}(S, N)=1 / 4$ in this simple example) on which the condition for lumpability is based.

More precisely, if the probability of moving from a micro state $\mathbf{x} \in X_{k}$ to a macro state $X_{l}$ is equal for all micro states in $X_{k}$, then all the information about the history which led to a particular state in $X_{k}$ is actually irrelevant, because from the macro perspective the future evolution is equivalent for any state in $X_{k}$. This leads to a condition on the transition matrix $\hat{P}$, namely, $\sum_{\mathbf{y} \in X_{l}} \hat{P}\left(\mathbf{x} \in X_{k}, \mathbf{y} \in X_{l}\right)$ must be equal for all $\mathbf{x} \in X_{k}$. For a process to be lumpable with respect to a partition $\mathbf{X}$, it is sufficient and necessary if this is true for any pair of sets $X_{k}, X_{l}$ of the partition. The respective theorem is presented in (Kemeny and Snell, 1976, Thm. 6.3.2) and we will come back to it with more detail and a focus on an application to ABMs in Sec. 3.2.3 (next chapter).

If the chain along with the desired state space partition is given, the application of the conditions provided in (Kemeny and Snell, 1976, Thm. 6.3.2) (as well as the subsequent matrix conditions) is relatively simple. However, if only the chain is given, it may be a real challenge to find partitions with respect to which the process is lumpable, not least due to the combinatorial 
explosion of the number of possible partitions. In this context, some algorithms have been presented for the task is to find the optimal or coarsest partition (Buchholz, 2000; Derisavi et al., 2003). Other authors have addressed these issues by studying the spectral properties of lumpable chains and have proposed algorithms based on that (Barr and Thomas, 1977; Meila and Shi, 2001; Takacs, 2006; Jacobi, 2008; Filliger and Hongler, 2008; Görnerup and Jacobi, 2010).

Another approach in which aggregate Markov chain descriptions are derived on the basis of model specifications that include the hierarchical and symmetric composition of sub-models has been followed by Buchholz (1995) and is also advised in the context of interactive Markov chains by Hermanns (1999); Hermanns and Katoen (2010). Namely for systems that »include a large number of identical and symmetric components « (Buchholz, 1995, 93/94), a reduced Markov chain description »resulting from exact lumping « (ibid., 94) is constructed directly during the modeling process. This avoids time-consuming (up to unfeasibility) computations on the huge transition matrices that the model would give rise to without the reduction. In this thesis, we formulate explicitly the complete microscopic system - containing all symmetries that come by the $\mathrm{ABM}$ at question - and lumpability arguments are based on that description (Secs. 3.1 and 3.2, next chapter). However, one of the main messages of this work concerns the translation of model symmetries into regularities on the associated micro chain which then enable lumpability. Especially Chapter 4, in which aggregate descriptions are derived starting from the symmetries of the agent network, is clearly related to the hierarchical approach due to Buchholz (1995) and the idea of symmetric composition in Hermanns (1999).

\subsubsection{Weak Lumpability}

This thesis mostly applies the strong version of lumpability described above in order to achieve a Markovian aggregation for ABMs. However, it is important to note that there is a weaker version of lumpability often referred to as weak lumpability which will play some role in the fifth chapter. While in the case of strong lumpability the projected process on $\mathbf{X}=\left\{X_{0}, X_{1}, \ldots\right\}$ is a Markov chain for any (initial) distribution, the weaker form of lumpability makes statements about the possibility to obtain a Markovian process at the aggregate level only for particular initial vectors.

For a description of the intuition behind weak lumpability the reader is encouraged to have a look to (Kemeny and Snell, 1976, Sec. 6.4., and pages 132/33 in particular) who themselves refer to Burke and Rosenblatt (1958) for some of their results. The main idea resides in the following possibility:

»Assume that no matter what the past information is, we always end up with the same assignment of probabilities for being in 
each of the states in $\left[X_{k}\right]$. Then again the past can have no influence on our predictions. « (Kemeny and Snell, 1976, 133)

A necessary and sufficient (though not always practical) condition (Kemeny and Snell, 1976, Thm. 6.4.1) is also provided, but the necessity and sufficiency of conditions for weak lumpability have also been subject of further discussion Abdel-Moneim and Leysieffer (1982); Rubino and Sericola (1989); Peng (1996).

On of the most important observations concerns the fact that if a regular chain is weakly lumpable with respect to a partition $\mathbf{X}$ for some probability vector, then it is weakly lumpable for the stationary vector (the left invariant vector of the transition matrix $\pi P=\pi$ ). See (Kemeny and Snell, 1976, Thm. 6.4.3) and also Rubino and Sericola (1989). This may be useful for the decision whether there is one distribution altogether for which a chain is weakly lumpable or not, (Kemeny and Snell, 1976, Thm. 6.4.4). This result has been extended to absorbing Markov chains by Ledoux et al. (1994). In the absorbing case, the quasi-stationary distribution is shown to play the role of the stationary vector which allows to relate the lumpability problem and existing algorithms for irreducible chains to the absorbing case.

\subsubsection{Nearly Lumpable and Non-Lumpable Aggregation}

It is well known that lumpability (the strong as well as the weak version) is rather an exception than the rule (Chazottes and Ugalde, 2003; Gurvits and Ledoux, 2005). Some form of aggregation, state space reduction, or macroscopic observation, however, is omnipresent in the analysis of complex systems and their dynamics. The question that then arises concerns the extend to which an aggregate process still informs us about the real microscopic model behavior.

There are some works that discuss these issues for the cases that the aggregation satisfies different types of lumpability. Namely, Schweitzer (1984); Sumita and Rieders (1989); Buchholz (1994) show that important stationary and transient measures are preserved by the lump. However, the direct derivation of stationary and transient properties of the original chain only by knowledge of the aggregated chain is possible only for a special case of weak lumpability referred to as exact lumpability (Buchholz, 1994, Thm. 3, Thm. $6)$. Buchholz (1994) also states that for any micro process and any partition it is possible to construct an aggregation that preserves the stationary measure. However, for the construction of this so-called ideal aggregate the stationary state of the original micro system has to be known. Though all lumpable aggregation are also ideal, the converse is not true and (Buchholz, 1994, 6) states:

»In all cases considered here, no information about the transient behaviour can be gained from the ideal aggregate. « 
In Chapter 5 of this work, we will construct an ideal non-lumpable aggregate for the contrarian VM on networks. While this thesis does not go much further in analyzing the relation between that ideal aggregate and the micro process, it does present an analytical example in which these questions can be addressed in the future.

A second important contribution due to Schweitzer (1984) and Buchholz (1994) is an operational concept of near lumpability. The main idea is that a nearly lumpable transition matrix $\hat{P}$ can be represented as $\hat{P}=\hat{A}+\epsilon \hat{B}$ where $\hat{A}$ is lumpable and $\epsilon$ is a sufficiently small constant used in analogy to its use in perturbation theory. Buchholz (1994) constructs bounding matrices for the transition probabilities that can be used to compute bounds for the stationary and transient quantities of the aggregated process. The computation of bounds in Buchholz (1994) is in part based on the work of Courtois and Semal (1984). See also Franceschinis and Muntz (1994); Dayar and Stewart (1997) for other concepts of nearly- or quasi-lumpability.

\subsubsection{Time-Series-Based Aggregation}

So far, we have mainly considered lumpability as a property of the transition matrix of the original chain, either directly or as induced by some (compositional) model. A useful complementary view on lumpability is provided by looking at it from an information-theoretic perspective, namely, in terms of the time series a model generates. A method to derive a Markovian state space aggregation on the basis of an information-theoretic view on time series data (typically created by some simple models) has been proposed by Görnerup and Jacobi (2008) and is inspired by the framework of computational mechanics (Crutchfield and Young, 1989; Shalizi and Crutchfield, 2001; Shalizi and Moore, 2003).

The approach in Görnerup and Jacobi (2008) starts from a sequence of symbols ( say $\bar{s}=\ldots, s_{t-1}, s_{t}, s_{t+1, \ldots}$ ) defined by some stochastic process on a finite alphabet $\boldsymbol{\Sigma}$. (For our purposes, we may assume that the sequence has been created by the microscopic Markov chain $(\boldsymbol{\Sigma}, \hat{P})$.) As usually in lumpability, consider further a projection map $\Pi: \boldsymbol{\Sigma} \rightarrow \mathbf{X}$ that induces a partition $\left(\mathbf{X}=\left\{X_{0}, X_{1}, \ldots, X_{n}\right\}\right)$ of the state space $\boldsymbol{\Sigma}$ by mapping sets of micro states in $\boldsymbol{\Sigma}$ into macro states $X_{k} \in \mathbf{X}$. By the projection map $\Pi$, the sequence $\bar{s}$ is transformed into a new sequence $\bar{x}=\Pi(\bar{s})=\ldots, x_{t-1}, x_{t}, x_{t+1}, \ldots$ with a new reduced alphabet set $\mathbf{X}$ (i.e., $x_{\tau}=\Pi\left(s_{\tau}\right) \in \mathbf{X}$ ). Now, in this context, lumpability of the process with respect to the partition $\mathbf{X}$ means that the macro sequence $\bar{x}$ exhibits the Markov property. In that case, Görnerup and Jacobi (2008) refer to the projection $\Pi$ as Markov projection, a notion that will be adopted throughout this thesis.

The decision whether the macro process $\bar{x}$ (obtained by a certain projection) is Markovian or not is based on the mutual information between the past $\left(\ldots, x_{t-2}, x_{t-1}\right)$ and the future $\left(x_{t+1}, x_{t+2}, \ldots\right)$ with respect to the 
present $\left(x_{t}\right)$. If the expected mutual information between past and future is zero, looking further back into the past does not provide any new information about the future evolution, that is, the future depends only on the present $x_{t}$ and $\bar{x}$ is a Markov process.

With its focus on time series and computations on it, the devision of a process or sequence into past, present and future, and the intense use of information theory, the approach due to Görnerup and Jacobi (2008) is located within a larger program called computational mechanics (Crutchfield and Young, 1989; Shalizi and Crutchfield, 2001; James et al., 2011, and references therein). The main idea in computational mechanics is to group histories which give rise to the same conditional probability distribution over futures into equivalence classes - so-called causal states - and to construct in this way a minimal causal model - called $\epsilon$-machines - for the prediction of the process at question. The reader may be referred to Shalizi and Crutchfield (2001) for an overview and several interesting theoretical results in computational mechanics. It is also noteworthy, in this context, that the particular role of Markovianity in the definition of macroscopic observables has been emphasized in Shalizi and Moore (2003). Finally, different concepts for the level identification in complex dynamical systems are compared and related in Pfante et al. (2013) who emphasize the particular role played by commutativity of aggregation and dynamics.

Time-series-based aggregation schemes as the one proposed by Görnerup and Jacobi (2008) as well as $\epsilon$-machines (Crutchfield and Young, 1989; Shalizi and Crutchfield, 2001) are appealing from the theoretical point of view, but their application to ABM aggregation is limited by their computational complexity (cf. Görnerup and Jacobi, 2008, 13). The fact that, even in very simple ABMs, the state space of the process to be handled becomes very large, challenges these approaches in two ways. The first one concerns the »combinatorial explosion « (ibid., 11) of the number of possible partitions, which is in fact a general difficulty in lumpability whenever the partition is not given a priori (see Sec. 2.4.1). More importantly, however, the larger the alphabet (and $\boldsymbol{\Sigma}$ becomes really large!), the more data must be generated and evaluated in order to obtain a workable approximation of the probability distribution of sequence blocks (cf. Shalizi and Crutchfield, 2001, Sec. VII.B/C). One way around this problem is to restrict to block size to one, as in Shalizi et al. (2004), which is actually exact if the original process is a Markov chain. Still, in this case, the number of states is huge and the estimation of the conditional probabilities (on the basis of which equivalence classes are constructed) requires a lot of simulation data.

\subsubsection{Aggregation in Dynamical Systems}

Finally, to complete this section, we should notice that aggregation and state space decomposition is a wide field which has been vividly discussed across 
different disciplines, during quite some time. In philosophy, it relates strongly to the more general discussions about the decomposability of a complex system (Simon, 1962) and from there to emergence (Wimsatt, 1986; Auger and Poggiale, 1998) and even further to the possible limitations of an reductionist account of complex systems (Wimsatt, 2006a). In economics, where much theory is in fact developed around aggregate measures, techniques for the aggregation of variables in dynamical systems have been developed (e.g., Theil, 1965; Simon and Ando, 1961; Ando and Fisher, 1963) as an operationalization »decomposability « and »nearly-decomposability « of a complex system mentioned above (Simon, 1962). These techniques have been transferred to theoretical biology, ecological modeling and population dynamics in particular, by Iwasa et al. (1987) in which conditions for exact aggregation in non-linear dynamical systems are given and Iwasa et al. (1989) which deals with approximate aggregations. The fact that the explicit consideration of more and more factors is a tendency in modern model development, has led to a renewed interest in aggregation techniques not only in Markov chains but also in the context of dynamical systems (see Auger et al., 2008 for a review of aggregation methods with application to population dynamics).

It is clear that aggregation techniques are actually relevant to all models which involve a large number of variables (or agents), in order to derive reduced model descriptions that might be amenable to analytical strategies. Markov chains and dynamical systems are probably the two most important mathematical formalisms to represent complex and high-dimensional systems that evolve in time. In this context, it is very interesting that methods for aggregation of variables in linear dynamical systems and lumpability in Markov chains can be based on the same principles, a fact that has recently been exploited in Jacobi and Görnerup (2009); Görnerup and Jacobi (2010).

\subsection{Motivation: Towards a Markov Chain Theory of Aggregation for Agent-Based Models}

\subsubsection{Bridging a Gap}

Though it has often been recognized that ABMs may be conceived as (stochastic) dynamical systems or Markov chains (Epstein and Axtell, 1996; Laubenbacher et al., 2009; Izquierdo et al., 2009; Page, 2012), the afore mentioned aggregation techniques developed for these systems have not yet been applied to ABMs. One of the reasons for this is that an explicit formulation of the micro process in terms of dynamical systems or Markov chains has been accomplished only in an abstract (Laubenbacher et al., 2009; Page, 2012) or approximate (Izquierdo et al., 2009) way. The explicit formalization of the micro process as a Markov chain - the reasoning presented in this thesis will be started with it (Sec. 3.1) - enables the application of the Markov chain theory of aggregation - that is, lumpability - to ABMs. 
The need for a mathematical framework that links the micro and the macro level has, of course, been noted earlier. For instance:

»Of course, microscopic and macroscopic theories are related, and understanding the connection between the two, e.g., through simulation or by deriving the latter from the former, is an important goal of any complex systems research.« (Lerman, 2001, 225)

Also the general possibility of applying mathematical aggregation techniques (Page, 2012) and complexity reduction by symmetry exploitation (Laubenbacher et al., 2009) has been noted, namely, in the context of dynamical systems and partly based on earlier work by Iwasa et al. (1987) in population ecology. However, a sophisticated and practicable mathematical framework for linking between micro and macro level processes in ABMs does not yet exist. This thesis is a first step to bridge this gap.

\subsubsection{The Micro-Macro Link}

The relation between the microscopic and the macroscopic has since long been subject for controversy. In sociology, it is manifest in the dichotomy of methodological individualism and structural functionalism. A good overview over the historical development of micro-macro debates from philosophy to social theory is provided in the introductory chapter (Alexander and Giesen, 1987) of a volume headed » The Micro-Macro Link « (Alexander et al., 1987).

$»$ The Micro-Macro Link « is a collection of essays by very influential social theorists in the micro as well as in the macro tradition about ways to overcome the micro-macro divide and link between the different levels of analysis. A synthetic formulation embracing the different levels from individual action to social order and back requires on the one hand a link from the micro to the macro pointing at questions related to various (from weaker to stronger) forms of emergence (Brodbeck, 1968; Giesen, 1987), aggregation and equilibrium (Coleman, 1987). On the other, it should also include concepts for the retro-action of the macro on the micro level, such as internalization (Parsons, 1954) or constraints on and the environment of individual actions (Alexander, 1987). One of the first acknowledged synthetic formulations of this linkage between micro and macro in sociology studies is from Max Weber (Weber, 1978) from where we quote the following basic observation:

$\gg$ within the realm of social action, certain empirical uniformities can be observed, that is, courses of action that are repeated by the actor or (simultaneously) occur among numerous actors « (Weber, 1978, 29) 
We shall see how a stylized version of this belief is incorporated in our study when passing from micro to macro dynamics.

ABMs are sometimes considered as a methodology to provide a $\gg$ theoretical bridge « (Macy and Willer, 2002, 148) between micro and macro theories (see also Saam, 1999; Squazzoni, 2008). Even if most of the models (especially the early ones) are actually a straight implementation of the individualistic program, there are some attempts to include into the model agents with some socio-cognitive abilities (see Squazzoni, 2008, 14-16) capable of the perception and internalization of the macro sphere. Also the experimentation with different interaction topologies can actually be seen as an attempt to understand the influence of social structure (macro) on the emergence of collective order (macro) transmitted through the level of individual interaction (micro).

Clearly, this thesis is not about social theory. It is about a mathematical technique to link micro dynamics to macro dynamics in models that may be designed on the basis of sociological theorizing. To my opinion, a well posed mathematical basis for these models may help the understanding of many of their observed properties, and it also provides a new perspective on aggregation and emergence and on how they are related. Linking the microdescription of an ABM to a macro-description in the form of a Markov chain provides information about the transition from the interaction of individual actors to the complex macroscopic behaviors observed in social systems. In particular, well-known conditions for lumpability (Sec. 2.4.1) make it possible to decide whether the macro model is still Markov. Conversely, this setting can also provide a suitable framework to understand the emergence of long range memory effects and patterns of spatial organization (Chapter 5).

\subsubsection{Computational Emergence and Aggregativity}

$\mathrm{ABM}$ and other related computational tools (such as $\mathrm{CA}$ ) play an increasingly important role also in the contemporary philosophical discussions of emergence. Some philosophers (e.g., Bedau, 1997, 2003; Huneman and Humphreys, 2008; Humphreys, 2008) advocate a position which makes use of computational models as a playground to address fundamental questions of emergence (see Symons, 2008 for a critical consideration). Questions about the relation of these artificial model environments to real phenomena are not ignored, but considered as an independent issue which is actually part of another debate. The field of computational emergence aims to establish »a close link between the concept of emergence and computation or computer simulations, which can perhaps be captured by the idea that an emergent phenomenon is one that arises from a computationally incompressible process « (Huneman and Humphreys, 2008, 425/26). The framework presented here provides explicit knowledge about the (in)compressibility of computational models and the dynamical processes which these models give rise to. 
While scientists use the term »emergence « relatively freely, the philosophical literature differentiates more carefully between different forms of emergence (ontological versus epistemological, strong versus weak, synchronic versus diachronic emergence) and the existence of some of these forms (ontological emergence in particular) is in fact highly controversial. In the context of computational models, emergence is often paraphrased by $\gg$ the whole is more than the sum of its parts « and an emergent property can be a certain macro-level pattern that could not be expected (and not predicted!) by looking at the micro level rules only. Along this lines, a well-known and explicitly computational account of weak emergence that fits the use of the term in complexity science has been offered by Bedau (1997, 2003):

$»$ The behavior of weakly emergent systems cannot be determined by any computation that is essentially simpler than the intrinsic natural computational process by which the system's behavior is generated.« (Bedau, 2003, 18)

Bedau (2003) uses CAs to illustrate these ideas and makes explicit reference to simulations: according to him a system property is emergent if it can be derived $\gg$ only by simulation « (ibid., 15).

An alternative position on emergence has been advocated by Wimsatt (1986) even before computer simulations became widespread. Wimsatt (1986) starts out from analyzing the conditions for a system property to be a mere aggregate of the properties of the parts of which the system is composed (see also Wimsatt, 2000, 2006a,b). Accordingly, a property of a system is called emergent if it does not satisfy these condition for aggregativity. In this way, Wimsatt is able to give a rather straightforward meaning to the dictum $\gg$ a complex system is more than the sum of its parts « by relating emergence to the lack of aggregativity. What makes Wimsatt's position particularly interesting for this work is not only that relation between aggregation and emergence, but also the observation expressed by the following statements:

$»[\mathrm{I}] \mathrm{t}$ is better to talk about properties of systems and their parts, and to analyze aggregativity as a kind of relation between these properties.« (Wimsatt, 1986, 260)

»Aggregativity and emergence concern the relationship between a property of a system under study and properties of its parts. « (Wimsatt, 2006a, 675)

The reason for which it is better to focus on properties, or rather to be explicit on that point, is that a system might be aggregative for one but emergent for another property. Just as a Markov chain might be lumpable with respect to one but non-lumpable with respect to another partition! 


\section{Agent-BAsed Models as Markov CHAINS}

This chapter spells out the most important theoretical ideas developed in this thesis. It first shows for a class of agent-based models (ABMs) that at the micro level they give rise to random walks on regular graphs. Secondly, it shows that observations of the models partition the state space of the micro chains such that micro configurations with the same observable value are projected into the same macro state. The conditions for the projected process to be again a Markov chain are given which relates the symmetry structure of the micro chains to the partition induced by macroscopic observables. The third and biggest part of this chapter is devoted to the analysis of a simple opinion model in order to illustrate these ideas. The projection from micro to macro emphasizes the particular role played by homogeneous mixing as a requirement for the Markovianity of the projected model. We present a Markov chain analysis of the binary voter model (VM) with a particular focus on its transient dynamics and show that the general VM can be reduced to the binary case by further projection. Finally, the question of interaction constraints in form of bounded confidence is addressed. Homogeneous interaction probabilities (homogeneous mixing) are assumed in all the analyses presented in this chapter. Interaction heterogeneities are left for the next chapters.

Most of the results presented in this chapter have been published in Banisch et al. (2012). In relation to this, the description of the micro process (Sec. 3.1) as well as the micro-macro projection (Sec. 3.2) contain much more details. The chapter also contains new material, the analysis of the transient with bounded confidence (Sec. 3.4.4) in particular. 


\subsection{The Micro Level}

\subsubsection{The Grammar of an ABM}

Let us consider an abstract ABM with finite configuration space $\boldsymbol{\Sigma}=\mathbf{S}^{N}$ (meaning that there are $N$ agents with attributes $x_{i} \in \mathbf{S}$ ). Any iteration of the model (any run of the ABM algorithm) maps a configuration $\mathbf{x} \in \boldsymbol{\Sigma}$ to another configuration $\mathbf{y} \in \boldsymbol{\Sigma}$. In general, the case that no agent changes such that $\mathbf{x}=\mathbf{y}$ is also possible. Let us denote such a mapping by $F_{z}: \boldsymbol{\Sigma} \rightarrow \boldsymbol{\Sigma}$ and denote the set of all possible mappings by $\mathcal{F}$. Notice that any element of $\mathcal{F}$ can be seen as a word of length $|\boldsymbol{\Sigma}|$ over an $|\boldsymbol{\Sigma}|$-ary alphabet, and there are $|\boldsymbol{\Sigma}|^{|\boldsymbol{\Sigma}|}$ such words (Flajolet and Odlyzko, 1990, 3).

Any $F_{z} \in \mathcal{F}$ induces a directed graph $\left(\boldsymbol{\Sigma}, F_{z}\right)$ the nodes of which are the elements in $\boldsymbol{\Sigma}$ (i.e., the agent configurations) and edges the set of ordered pairs $\left(\mathbf{x}, F_{z}(\mathbf{x})\right), \forall \mathbf{x} \in \mathbf{\Sigma}$. Such a graph is called functional graph of $F_{z}$ because it displays the functional relations of the map $F_{z}$ on $\boldsymbol{\Sigma}$. That is, it represents the logical paths induced by $F_{z}$ on the space of configurations for any initial configuration $\mathbf{x}$.

Each iteration of an ABM can be thought of as a stochastic choice out of a set of deterministic options. For an ABM in a certain configuration $\mathbf{x}$, there are usually several options (several $\mathbf{y}$ ) to which the algorithm may lead with a well-defined probability (see Sec. 2.2). Therefore, in an ABM, the transitions between the different configurations $\mathbf{x}, \mathbf{y}, \ldots \in \boldsymbol{\Sigma}$ are not defined by one single map $F_{z}$, but there is rather a subset $\mathcal{F}_{Z} \subset \mathcal{F}$ of maps out of which one map is chosen at each time step with certain probability. Let us assume we know all the mappings $\mathcal{F}_{Z}=\left\{F_{1}, \ldots, F_{z}, \ldots, F_{n}\right\}$ that are realized by the ABM of our interest. With this, we are able to define a functional graph representation by $\left(\boldsymbol{\Sigma}, \mathcal{F}_{Z}\right)$ which takes as the nodes all elements of $\boldsymbol{\Sigma}$ (all agent configurations) and an $\operatorname{arc}(\mathbf{x}, \mathbf{y})$ exists if there is at least one $F_{z} \in \mathcal{F}_{Z}$ such that $F_{z}(\mathbf{x})=\mathbf{y}$. This graph defines the »grammar « of the system for it displays all the logically possible transitions between any pair of configurations of the model.

Consider the VM with three agents as an example. In the VM agents have two possible states $(\mathbf{S}=\{\square, \boldsymbol{\square}\})$ and the configuration space for a model of three agents is $\boldsymbol{\Sigma}=\{\square, \boldsymbol{\square}\}^{3}$. In the iteration process, one agent $i$ is chosen at random along with one of its neighbors $j$ and agent $i$ imitates the state of $j$. This means that $y_{i}=x_{j}$ after the interaction event. Notice that once an agent pair $(i, j)$ is chosen the update is defined by a deterministic map $\mathbf{u}: \mathbf{S}^{2} \rightarrow \mathbf{S}$. Stochasticity enters first because of the random choice of $i$ and second through the random choice of one agent in the neighborhood. Let us look at an example with three agents in the configuration $\mathbf{x}=(\square \mathbf{\square})$. If the first agent is chosen $\left(i=1\right.$ and $\left.x_{1}=\square\right)$ then this agent will certainly change state to $y_{1}=\boldsymbol{\square}$ because it will in any case meet a black agent. For the second and the third agent $(i=2$ or $i=3)$ the update result 
depends on whether one or the other neighbor is chosen because they are in different states. Noteworthy, different agent choices may lead to the same configuration. Here, this is the case if the agent pair $(2,3)$ or $(3,2)$ is chosen in which case the agent ( 2 or 3 ) does not change its state because $x_{2}=x_{3}$. Therefore we have $\mathbf{y}=\mathbf{x}$ and there are two paths realizing that transition.

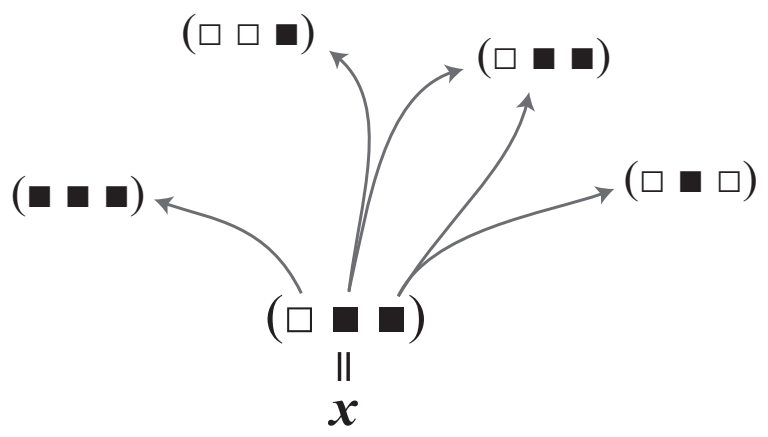

Figure 3.1: Possible paths from configuration $\mathbf{x}=(\square \mathbf{\square})$ in a small VM of three agents.

In practice, the explicit construction of the entire functional graph may rapidly become a tedious task due to the huge dimension of the configuration space and the fact that one needs to check if $F_{z}(\mathbf{x})=\mathbf{y}$ for each mapping $F_{z} \in \mathcal{F}_{Z}$ and all pairs of configurations $\mathbf{x}, \mathbf{y}$. On the other hand, the main interest here is a theoretical one, because, as a matter of fact, a representation as a functional graph of the form $\Gamma=\left(\boldsymbol{\Sigma}, \mathcal{F}_{Z}\right)$ exists for any model that comes in form of a computer algorithm. It is therefore a quite general way of formalizing ABMs and, as we will see in the sequel, allows under some conditions to verify the Markovianity of the models at the micro level.

If we really want to construct the »grammar « of an ABM explicitly this requires the dissection of stochastic and deterministic elements of the iteration procedure of the model. As an example, let us consider again the VM for which such a dissection is not difficult. In the VM, the random part consists of the choice of two connected agents $(i, j)$. Once this choice is made we know that $y_{i}=x_{j}$ by the interaction rule. This is sufficient to derive the functional representation of the VM, because we need only to check one by one for all possible choices $(i, j)$ which transitions this choice induces on the configuration space. For a system of three agents, with all agents connected to the other two, the set of functions $\mathcal{F}_{Z}=\left\{F_{1}, \ldots, F_{z}, \ldots, F_{n}\right\}$ is specified in Table 3.1. Notice that with three agents, there are 8 possible configurations indexed here by $a, b, \ldots, h$. Moreover, there are 6 possible choices for $(i, j)$ such that $\mathcal{F}_{Z}$ consists of $n=6$ mappings.

Each row of the table represents a mapping $F_{z}: \boldsymbol{\Sigma} \rightarrow \boldsymbol{\Sigma}$ by listing to which configuration $\mathbf{y}$ the respective map takes the configurations $a$ to $h$. The first row, to make an example, represents the choice of the agent 


\begin{tabular}{|c|c|c|c|c|c|c|c|c|c|}
\hline$z$ & $(i, j)$ & $\mathbf{\square}^{\mathrm{a}}$ & $\stackrel{b}{\mathbf{b}^{\circ}}$ & $\begin{array}{c}\mathrm{c} \\
\square \\
\square\end{array}$ & ${ }^{\mathrm{d}}$ & $\begin{array}{c}\mathrm{e} \\
\square \square \square\end{array}$ & $\begin{array}{c}\mathrm{f} \\
\square \square\end{array}$ & $\begin{array}{c}\mathrm{g} \\
\square \square\end{array}$ & $\begin{array}{c}\mathrm{h} \\
\square \square \square\end{array}$ \\
\hline 1 & $(1,2)$ & $\mathrm{a}$ & $\mathrm{b}$ & $\mathrm{g}$ & $\mathrm{a}$ & $\mathrm{h}$ & $\mathrm{b}$ & $\mathrm{g}$ & $\mathrm{h}$ \\
\hline 2 & $(1,3)$ & a & $\mathrm{f}$ & c & a & h & f & c & $\mathrm{h}$ \\
\hline 3 & $(2,1)$ & $\mathrm{a}$ & b & a & $\mathrm{g}$ & b & $\mathrm{h}$ & $\mathrm{g}$ & $\mathrm{h}$ \\
\hline 4 & $(3,1)$ & $\mathrm{a}$ & $\mathrm{a}$ & $\mathrm{c}$ & $\mathrm{f}$ & $\mathrm{c}$ & $\mathrm{f}$ & $\mathrm{h}$ & $\mathrm{h}$ \\
\hline 5 & $(2,3)$ & $\mathrm{a}$ & e & a & d & e & $\mathrm{h}$ & d & $\mathrm{h}$ \\
\hline 6 & $(3,2)$ & $\mathrm{a}$ & a & e & d & e & d & $\mathrm{h}$ & $\mathrm{h}$ \\
\hline
\end{tabular}

Table 3.1: $\mathcal{F}_{Z}$ for the VM with three agents.

pair $(1,2)$. The changes this choice induces depend on the actual agent configuration $\mathbf{x}$. Namely, for any $\mathbf{x}$ with $x_{1}=x_{2}$ we have $F_{1}(\mathbf{x})=F_{(1,2)}(\mathbf{x})=$ $\mathbf{x}$. So the configurations $a, b, g, h$ are not changed by $F_{(1,2)}$. For the other configurations it is easy to see that $(\square \square) \rightarrow(\square \square \square)(c \rightarrow g),(\square \square \mathbf{\square}) \rightarrow$ (घ口) $(d \rightarrow a),(\mathbf{\square} \square) \rightarrow(\square \square \square)(e \rightarrow h)$, and $(\square \square) \rightarrow(\square \square)(f \rightarrow$ $b)$. Notice that the two configurations $(\square \square \square)$ and ( $\square \square$ ) with all agents equal are not changed by any map and correspond therefore to the final configurations of the VM.

In Fig. 3.2, the complete functional graph $\Gamma=\left(\boldsymbol{\Sigma}, \mathcal{F}_{Z}\right)$ of the VM with three agents is shown. This already gives us some important information about the behavior of the VM such as the existence of two final configurations with all agents in the same state. We also observe that the VM iteration gives rise to a very regular functional graph, namely, the $N$-dimensional hypercube. In what follows, we show how to derive the respective transition probabilities associated to the arrows in Fig. 3.2.

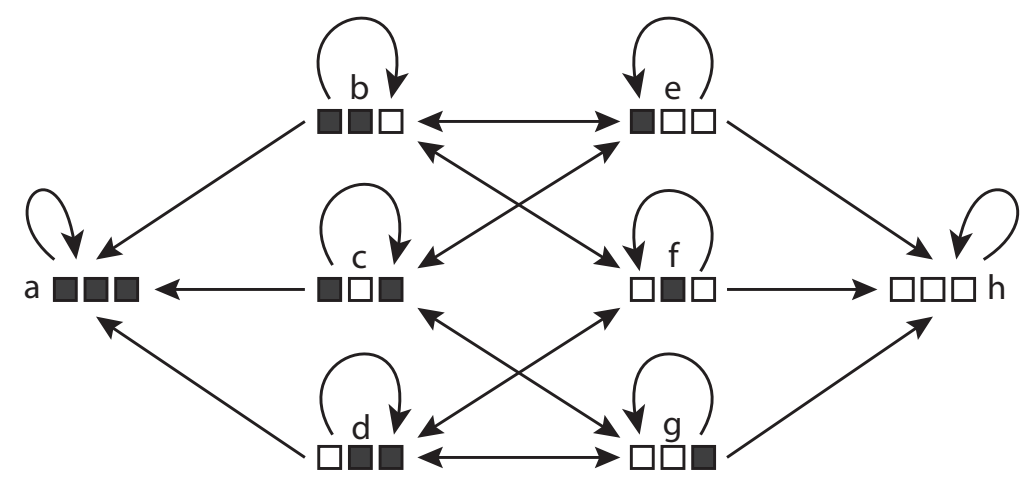

Figure 3.2: Grammar of the VM with three agents.

\subsubsection{From Functional Graphs to Markov Chains}

A functional graph $\Gamma=\left(\boldsymbol{\Sigma}, \mathcal{F}_{Z}\right)$ defines the $»$ grammar $«$ of an ABM in the sense that it shows all possible transitions enabled by the model. It is the 
first essential step in the construction of the Markov chain associated with the ABM at the micro level because there is a non-zero transition probability only if there is an arrow in the functional graph. Consequently, all that is missing for a Markov chain description is the computation of the respective transition probabilities.

For a class of models, including the VM, this is relatively simple because we can derive a random mapping representation (Levin et al., 2009, 6/7) directly from the ABM rules. Namely, if $F_{z_{1}}, F_{z_{2}}, \ldots$ is a sequence of independent random maps, each having the same distribution $\omega$, and $S_{0} \in \boldsymbol{\Sigma}$ has distribution $\mu_{0}$, then the sequence $S_{0}, S_{1}, \ldots$ defined by

$$
S_{t}=F_{z_{t}}\left(S_{t-1}\right), t \geq 1
$$

is a Markov chain on $\boldsymbol{\Sigma}$ with transition matrix $\hat{P}$ :

$$
\hat{P}(\mathbf{x}, \mathbf{y})=\operatorname{Pr}_{\omega}\left[z, F_{z}(\mathbf{x})=\mathbf{y}\right] ; \mathbf{x}, \mathbf{y} \in \mathbf{\Sigma} .
$$

Conversely (Levin et al. (2009)), any Markov chain has a random map representation (RMR). Therefore, in that case, (3.1) and (3.2) may be taken as an equivalent definition of a Markov chain. This is particularly useful in our case, because it shows that an ABM which can be described as above is, from a mathematical point of view, a Markov chain. This includes several models described in Izquierdo et al. (2009).

In the VM, the separation of stochastic and deterministic elements is clear-cut and therefore a random mapping representation is obtained easily. As already shown in Table 3.1, we can use the possible agent choices $(i, j)$ directly to index the collection of maps $F_{(i, j)} \in \mathcal{F}_{Z}$. We denote as $\omega(i, j)$ the probability of choosing the agent pair $(i, j)$ which corresponds to choosing the map $F_{(i, j)}$. It is clear that we can proceed in this way in all models where the stochastic part concerns only the choice of agents. Then, the distribution $\omega$ is independent of the current system configuration and the same for all times $\left(\omega\left(z_{t}\right)=\omega(z)\right)$. In this case, we obtain for the transition probabilities

$$
\hat{P}(\mathbf{x}, \mathbf{y})=\operatorname{Pr}_{\omega}\left[(i, j), F_{(i, j)}(\mathbf{x})=\mathbf{y}\right]=\sum_{\substack{(i, j): \\ F_{(i, j)}(\mathbf{x})=\mathbf{y}}} \omega(i, j) .
$$

That is, the probability of transition from $\mathbf{x}$ to $\mathbf{y}$ is the conjoint probability $\sum \omega(i, j)$ of choosing an agent pair $(i, j)$ such that the corresponding map takes $\mathbf{x}$ to $\mathbf{y}$ (i.e., $F_{(i, j)}(\mathbf{x})=\mathbf{y}$ ).

\subsubsection{Single-Step Dynamics}

In this thesis, we focus on a class of models which we refer to as single-step dynamics. They are characterized by the fact that only one agent changes at a time step. Notice that this is very often the case in ABMs with a 
sequential update scheme and that sequential update is, as a matter of fact, the most typical iteration scheme in ABMs. In terms of the »grammar « of these models, this means that non-zero transition probabilities are only possible between system configuration that differ in at most one position. And this gives rise to random walks on regular graphs.

Consider a set of $N$ agents each one characterized by individual attributes $x_{i}$ that are taken in a finite list of possibilities $\mathbf{S}=\{1, \ldots, \delta\}$. In this case, the space of possible agent configurations is $\boldsymbol{\Sigma}=\mathbf{S}^{N}$. Consider further a deterministic update function $\mathbf{u}: \mathbf{S}^{r} \times \Lambda \rightarrow \mathbf{S}$ which takes configuration $\mathbf{x} \in \boldsymbol{\Sigma}$ at time $t$ to configuration $\mathbf{y} \in \boldsymbol{\Sigma}$ at $t+1$ by

$$
y_{i}=\mathbf{u}\left(x_{i}, x_{j}, \ldots, x_{k}, \lambda\right) .
$$

To go from one time step to the other in agent systems, usually, an agent $i$ is chosen first to perform a step. The decision of $i$ then depends on its current state $\left(x_{i}\right)$ and the attributes of its neighbors $\left(x_{j}, \ldots, x_{k}\right)$. The finite set $\Lambda$ accounts for a possible stochastic part in the update mechanism such that different behavioral options are implemented by different update functions $\mathbf{u}\left(\ldots, \lambda_{1}\right), \mathbf{u}\left(\ldots, \lambda_{2}\right)$ etc. Notice that for the case in which the attributes of the agents $\left(x_{i}, x_{j}, \ldots, x_{k}\right)$ uniquely determine the agent decision we have $\mathbf{u}: \mathbf{S}^{r} \rightarrow \mathbf{S}$ which strongly resembles the update rules implemented in cellular automata $(\mathrm{CA})$.

As opposed to classical CA, however, a sequential update scheme is used in the class of models considered here. In the iteration process, first, a random choice of agents along with a $\lambda$ to index the possible behavioral options is performed with probability $\omega(i, j, \ldots, k, \lambda)$. This is followed by the application of the update function which leads to the new state of agent $i$ by Eq. (3.4).

Due to the sequential application of an update rule of the form $\mathbf{u}: \mathbf{S}^{r} \times$ $\Lambda \rightarrow \mathbf{S}$ only one agent (namely agent $i$ ) changes at a time so that all elements in $\mathbf{x}$ and $\mathbf{y}$ are equal except that element which corresponds to the agent that was updated during the step from $\mathbf{x}$ to $\mathbf{y}$. Therefore, $x_{j}=y_{j}, \forall j \neq i$ and $x_{i} \neq y_{i}$. We call $\mathbf{x}$ and $\mathbf{y}$ adjacent and denote this by $\mathbf{x} \stackrel{i}{\sim} \mathbf{y}$.

It is then also clear that a transition from $\mathbf{x}$ to $\mathbf{y}$ is possible if $\mathbf{x} \sim \mathbf{y}$. Therefore, the adjacency relation $\sim$ defines the $» \operatorname{grammar} \ll \Gamma_{S S D}$ of the entire class of single-step models. Namely, the existence of a map $F_{z}$ that takes $\mathbf{x}$ to $\mathbf{y}, \mathbf{y}=F_{z}(\mathbf{x})$, implies that $\mathbf{x} \stackrel{i}{\sim} \mathbf{y}$ for some $i \in\{1, \ldots, N\}$. This means that any ABM that belongs to the class of single-step models performs a walk on $\Gamma_{S S D}$ or on a subgraph of it.

Let us briefly consider the structure of the graph $\Gamma_{S S D}$ associated to the entire class of single-step models. From $\mathbf{x} \stackrel{i}{\sim} \mathbf{y}$ for $i=1, \ldots, N$ we know that for any $\mathbf{x}$, there are $\delta N$ different vectors $\mathbf{y}$ which differ from $\mathbf{x}$ in a single position, where $\delta$ is the number of possible agent attributes. Therefore, $\Gamma_{S S D}$ is a regular graph with degree $\delta N+1$, because in our case, the system may 
loop by $y_{i}=x_{i}$. As a matter of fact, our definition of adjacency as $\gg$ different in one position of the configuration « is precisely the definition of so-called Hamming graphs which tells us that $\Gamma_{S S D}=H(N, \delta)$ (with loops). In the case of the VM, where $\delta=2$ we find $H(N, 2)$ which corresponds to the $N$-dimensional hypercube.

As before, the transition probability matrix of the micro chain is denoted by $\hat{P}$ with $\hat{P}(\mathbf{x}, \mathbf{y})$ being the probability for the transition from $\mathbf{x}$ to $\mathbf{y}$. The previous considerations tell us that non-zero transition probabilities can exist only between two configurations that are linked in $H(N, d)$ plus the loop $(\hat{P}(\mathbf{x}, \mathbf{x}))$. Therefore, each row of $\hat{P}$ contains no more than $\delta N+1$ nonzero entries. In the computation of $\hat{P}$ we concentrate on pairs of adjacent configurations. For $\mathbf{x} \stackrel{i}{\sim} \mathbf{y}$ with $x_{i} \neq y_{i}$ we have

$$
\hat{P}(\mathbf{x}, \mathbf{y})=\sum_{\substack{(i, j, \ldots, k, \lambda): \\ y_{i}=\mathbf{u}\left(x_{i}, x_{j}, \ldots, x_{k}, \lambda\right)}} \omega(i, j, \ldots, k, \lambda)
$$

which is the conjoint probability to choose agents and a rule $(i, j, \ldots, k, \lambda)$ such that the $i$ th agent changes its attribute by $y_{i}=\mathbf{u}\left(x_{i}, x_{j}, \ldots, x_{k}, \lambda\right)$. For the probability that the model remains in $\mathbf{x}, \hat{P}(\mathbf{x}, \mathbf{x})$, we have

$$
\hat{P}(\mathbf{x}, \mathbf{x})=1-\sum_{\mathbf{y} \sim \mathbf{x}} \hat{P}(\mathbf{x}, \mathbf{y})
$$

Eq. (3.5) makes visible that the probability distribution $\omega$ plays the crucial role in the computation of the elements of $\hat{P}$.

The VM is a very simple instance of single-step dynamics. The update function is given by $y_{i}=\mathbf{u}\left(x_{i}, x_{j}\right)=x_{j}$ and the stochastic part of the model concerns only the choice of an agent pair $(i, j)$ with probability $\omega(i, j)$. For adjacent configuration with $\mathbf{x} \stackrel{i}{\sim} \mathbf{y}$, Eq. (3.5) simplifies to

$$
\hat{P}(\mathbf{x}, \mathbf{y})=\sum_{j:\left(y_{i}=\mathbf{u}\left(x_{i}, x_{j}\right)\right)} \omega(i, j)=\sum_{j:\left(y_{i}=x_{j}\right)} \omega(i, j)
$$

Notice that (3.7) is applicable to all ABMs in which first an agent pair $(i, j)$ is chosen at random and second a deterministic update rule $y_{i}=$ $\mathbf{u}\left(x_{i}, x_{j}\right)$ defines the outcome of the interaction between $i$ and $j$. For a binary attribute space $\mathbf{S}=\{\square, \boldsymbol{\square}\}$ some possible update rules $\mathbf{u}: \mathbf{S} \times \mathbf{S} \rightarrow \mathbf{S}$ are shown in Table 3.2 below.

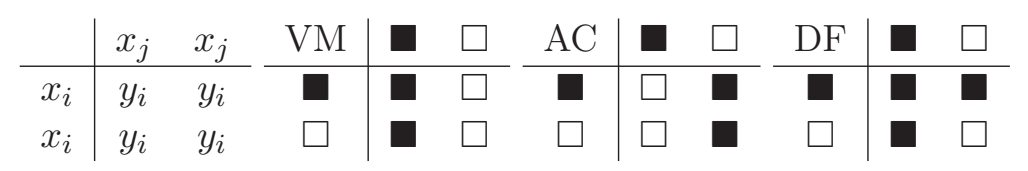

Table 3.2: Update rules $y_{i}=\mathbf{u}\left(x_{i}, x_{j}\right)$ for the voter model (VM), antiferromagnetic coupling (AC) and diffusion (DF). 


\subsection{Macrodynamics, Projected Systems and Ob- servables}

\subsubsection{Micro and Macro in ABMs}

What do we look at when we analyze an ABM? Typically, we try to capture the dynamical behavior of a model by studying the time evolution of parameters or indicators that inform us about the global state of the system. Although, in some cases, we might understand the most important dynamical features of a model by looking at repeated visualizations of all details of the agent system through time, basic requirements of the scientific method will eventually enforce a more systematic analysis of the model behavior in the form of systematic computational experiments and $»$ extensive sensitivity analysis « (Epstein, 2006, 28). In this, there is no other choice than to leave the micro level of all details and to project the system behavior or state onto global structural indicators representing the system as a whole. In many cases, a description like that will even be desired, because the focus of attention in ABMs, the facts to be explained, are usually at a higher macroscopic level beyond the microscopic description. In fact, the search for microscopic foundations for macroscopic regularities has been an integral motivation in the development of ABMs (see Macy and Willer, 2002; Squazzoni, 2008).

It is characteristic of any such macroscopic system property that it is invariant with respect to certain details of the agent configuration. In other words, any observation defines, in effect, a many-to-one relation by which sets of micro configurations with the same observable value are subsumed into the same macro state. Consider the population dynamics in the sugarscape model by Epstein and Axtell (1996) as an example. The macroscopic indicator is, in this case, the number of agents $N$. This aggregate value is not sensitive with respect to the exact positions (the sites) at which the agents are placed, but only to how many sites are occupied. Consequently, there are many possible configurations of agent occupations in the sugarspace with an equal number of agents $N$ and all of them correspond to the same macro state. Another slightly more complicated example is the skewed wealth distribution in the sugarscape model. It is not important which agents contribute to each specific wealth (sugar) level, but only how many there are in each level. This describes how macro descriptions of ABMs are related to observations, system properties, order parameters and structural indicators, and it also brings into the discussion to the concepts of aggregation and decomposition.

Namely, aggregation is one way (in fact, a very common one) of realizing such a many-to-one mapping from micro-configurations to macroscopic system properties and observables. For simple models of opinion dynamics inspired by spin physics, for instance, it is very common to use the average opinion - due to the spin analogy often called »system magnetization« - as 
an order parameter and to study the system behavior in this way. Magnetization, computed by summation over the spins and division by the total number of spins, is a true aggregative measure. Magnetization levels or values are then used to classify spin or opinion configurations, such that those configurations with the same magnetization value correspond to the same macro state. This many-to-one mapping of sets of micro configurations onto macro states automatically introduces a decomposition of the state space at the micro level $\boldsymbol{\Sigma}$.

\subsubsection{Observables, Partitions and Projected Systems}

The formulation of an ABM as a Markov chain developed in the previous section allows a formalization of this micro-macro link in terms of projections. Namely, a projection of a Markov chain with state space $\boldsymbol{\Sigma}$ is defined by a new state space $\mathbf{X}$ and a projection map $\Pi$ from $\boldsymbol{\Sigma}$ to $\mathbf{X}$. The meaning of the projection $\Pi$ is to lump sets of micro configurations in $\boldsymbol{\Sigma}$ according to some macro property in such a way that, for each $X \in \mathbf{X}$, all the configurations of $\boldsymbol{\Sigma}$ in $\Pi^{-1}(\mathbf{X})$ share the same property.

Therefore, such projections are important when catching the macroscopic properties of the corresponding ABM because they are in complete correspondence with a classification based on an observable property of the system. To see how this correspondence works let us suppose that we are interested in some factual property of our agent-based system. This means that we are able to assign to each configuration the specific value of its corresponding property. Regardless of the kind of value used to specify the property (qualitative or quantitative), the set $\mathbf{X}$ needed to describe the configurations with respect to the given property is a finite set, because the set of all configurations is also finite. Let then $\phi: \Sigma \rightarrow \mathbf{X}$ be the function that assigns to any configuration $\mathbf{x} \in \boldsymbol{\Sigma}$ the corresponding value of the considered property. It is natural to call such $\phi$ an observable of the system. Now, any observable of the system naturally defines a projection $\Pi$ by lumping the set of all the configurations with the same $\phi$ value. Conversely any (projection) map $\Pi$ from $\boldsymbol{\Sigma}$ to $\mathbf{X}$ defines an observable $\phi$ with values in the image set $\mathbf{X}$. Therefore these two ways of describing the construction of a macro-dynamics are equivalent and the choice of one or the other point of view is just a matter of taste.

The price to pay in passing from the micro to the macro-dynamics in this sense (Kemeny and Snell, 1976; Chazottes and Ugalde, 2003) is that the projected system is, in general, no longer a Markov chain: long memory (even infinite) may appear in the projected system. This »complexification « of the macro dynamics with respect to the micro dynamics is a fingerprint of dynamical emergence in agent-based and other computational models (cf. Humphreys, 2008). 


\subsubsection{Lumpability and Symmetry}

Under certain conditions, the projection of a Markov chain $(\boldsymbol{\Sigma}, \hat{P})$ onto a coarse-grained partition $\mathbf{X}$, obtained by aggregation of states, is still a Markov chain. In Markov chain theory this is known as lumpability (or strong lumpability), and necessary and sufficient conditions for this to happen are known. Let us restate the respective Thm. 6.3.2 of Kemeny and Snell (1976) using our notations, where $\boldsymbol{\Sigma}$ denotes the configuration space of the micro chain and $\hat{P}$ the respective transition matrix, and $\mathbf{X}=\left(X_{1}, \ldots, X_{r}\right)$ is a partition of $\mathbf{\Sigma}$. Let $\hat{p}_{\mathbf{x} Y}=\sum_{\mathbf{y} \in Y} \hat{P}(\mathbf{x}, \mathbf{y})$ denote the conjoint probability for $\mathbf{x} \in \boldsymbol{\Sigma}$ to go to the set of elements $\mathbf{y} \in Y$ where $Y \subseteq \boldsymbol{\Sigma}$ is a subset of the configuration space.

Theorem 3.2.1 (Kemeny and Snell, 1976, 124) A necessary and sufficient condition for a Markov chain to be lumpable with respect to a partition $\mathbf{X}=$ $\left(X_{1}, \ldots, X_{r}\right)$ is that for every pair of sets $X_{i}$ and $X_{j}, \hat{p}_{\mathbf{x} X_{j}}$ have the same value for every $\mathbf{x}$ in $X_{i}$. These common values $\left\{\hat{p}_{i j}\right\}$ form the transition matrix for the lumped chain.

In general it may happen that, for a given Markov chain, some projections are Markov and others not. Therefore a judicious choice of the macro properties to be studied may help the analysis.

In order to establish the lumpability in the cases of interest we shall use symmetries of the model. For further convenience, we state a result for which the proof is easily given Thm. 6.3.2 of Kemeny and Snell (1976):

Theorem 3.2.2 Let $(\boldsymbol{\Sigma}, \hat{\mathbf{P}})$ be a Markov chain and $\mathbf{X}=\left(X_{1}, \ldots, X_{n}\right)$ a partition of $\boldsymbol{\Sigma}$. Suppose that there exists a group $\mathcal{G}$ of bijections on $\boldsymbol{\Sigma}$ that preserve the partition $\left(\forall \mathbf{x} \in X_{i}\right.$ and $\forall \hat{\sigma} \in \mathcal{G}$ we have $\left.\hat{\sigma}(\mathbf{x}) \in X_{i}\right)$. If the Markov transition probability $\hat{P}$ is symmetric with respect to $\mathcal{G}$,

$$
\hat{P}(\mathbf{x}, \mathbf{y})=\hat{P}(\hat{\sigma}(\mathbf{x}), \hat{\sigma}(\mathbf{y})): \forall \hat{\sigma} \in \mathcal{G},
$$

the partition $\left(X_{1}, \ldots, X_{n}\right)$ is (strongly) lumpable.

Proof. For the proof it is sufficient to show that any two configurations $\mathbf{x}$ and $\mathbf{x}^{\prime}$ with $\mathbf{x}^{\prime}=\hat{\sigma}(\mathbf{x})$ satisfy

$$
\hat{p}_{\mathbf{x} Y}=\sum_{\mathbf{y} \in Y} \hat{P}(\mathbf{x}, \mathbf{y})=\sum_{\mathbf{y} \in Y} \hat{P}\left(\mathbf{x}^{\prime}, \mathbf{y}\right)=\hat{p}_{\mathbf{x}^{\prime} Y}
$$

for all $Y \in \mathbf{X}$. Consider any two subsets $X, Y \in \mathbf{X}$ and take $\mathbf{x} \in X$. Because $\mathcal{G}$ preserves the partition it is true that $\mathbf{x}^{\prime} \in X$. Now we have to show that Eq. (3.9) holds. First the probability for $\mathbf{x}^{\prime}=\hat{\sigma}(\mathbf{x})$ to go to an element $\mathbf{y} \in Y$ is

$$
\hat{p}_{\hat{\sigma}(\mathbf{x}) Y}=\sum_{\mathbf{y} \in Y} \hat{P}(\hat{\sigma}(\mathbf{x}), \mathbf{y})
$$


Because the $\hat{\sigma}$ are bijections that preserve the partition $\mathbf{X}$ we have $\hat{\sigma}(Y)=Y$ and there is for every $\mathbf{y} \in Y$ exactly one $\hat{\sigma}(\mathbf{y}) \in Y$. Therefore we can substitute

$$
\hat{p}_{\hat{\sigma}(\mathbf{x}) Y}=\sum_{\mathbf{y} \in Y} \hat{P}(\hat{\sigma}(\mathbf{x}), \hat{\sigma}(\mathbf{y}))=\sum_{\mathbf{y} \in Y} \hat{P}(\mathbf{x}, \mathbf{y})=\hat{p}_{\mathbf{x} Y},
$$

where the second equality comes by the symmetry condition (3.8) that $\hat{P}(\mathbf{x}, \mathbf{y})=\hat{P}(\hat{\sigma}(\mathbf{x}), \hat{\sigma}(\mathbf{y}))$.

The usefulness of the conditions for lumpability stated in Thm. 3.2.2 becomes apparent recalling that ABMs can be seen as random walks on regular graphs defined by the functional graph or $»$ grammar $\ll$ of the model $\Gamma=\left(\boldsymbol{\Sigma}, \mathcal{F}_{Z}\right)$. The full specification of the random walk $(\boldsymbol{\Sigma}, \hat{P})$ is obtained by assigning transition probabilities to the connections in $\Gamma$ and we can interpret this as a weighted graph. The regularities of $(\boldsymbol{\Sigma}, \hat{P})$ are captured by a number of non-trivial automorphisms which, in the case of ABMs, reflect the symmetries of the models.

In fact, Thm. 3.2.2 allows to systematically exploit the symmetries of an agent model in the construction of partitions with respect to which the micro chain is lumpable. Namely, the symmetry requirement in Thm. 3.2.2, that is, Eq. (3.8), corresponds precisely to the usual definition of automorphisms of $(\boldsymbol{\Sigma}, \hat{P})$. The set of all permutations $\hat{\sigma}$ that satisfy (3.8) corresponds then to the automorphism group of $(\boldsymbol{\Sigma}, \hat{P})$.

Lemma 3.2.3 Let $\mathcal{G}$ be the automorphism group of the micro chain $(\boldsymbol{\Sigma}, \hat{P})$. The orbits of $\mathcal{G}$ define a lumpable partition $\mathbf{X}$ such that every pair of micro configurations $\mathbf{x}, \mathbf{x}^{\prime} \in \mathbf{\Sigma}$ for which $\exists \hat{\sigma} \in \mathcal{G}$ such that $\mathbf{x}^{\prime}=\hat{\sigma}(\mathbf{x})$ belong to the same subset $X_{i} \in \mathbf{X}$.

Remark 3.2.1 Lemma 3.2.3 actually applies to any $\mathcal{G}$ that is a proper subgroup of the automorphism group of $(\boldsymbol{\Sigma}, \hat{P})$. The basic requirement for such a subset $\mathcal{G}$ to be a group is that be closed under the group operation which establishes that $\hat{\sigma}\left(X_{i}\right)=X_{i}$. With the closure property, it is easy that any such subgroup $\mathcal{G}$ defines a lumpable partition in the sense of Thm. 3.2.2.

\subsection{Opinion Dynamics and Projected Systems}

Voter-like models - here we shall interpret them as models of opinion and social dynamics - provide nice examples where such a projection construction is particularly meaningful. If there are $\delta$ possible agent attributes, we consider the projection $\Pi$ that maps each $\mathbf{x} \in \boldsymbol{\Sigma}$ into a macro state $X_{\left\langle k_{1}, \ldots, k_{\delta}\right\rangle} \in \mathbf{X}$ where $k_{s}, s=1, \ldots, \delta$, is the number of agents in $\mathbf{x}$ with attribute $s$. This captures the model dynamics in terms of frequencies of all $\delta$ attributes. The projected configuration space is then made of the $X_{\left\langle k_{1}, \ldots, k_{\delta}\right\rangle}$ where $k_{s} \geq 0$, $s=1, \ldots, \delta$ and $\sum_{1}^{\delta} k_{s}=N$. We shall now treat in detail the VM as an example of the previous ideas. 


\subsubsection{The Macro Dynamics of the Binary Voter Model}

The case of a binary opinion model, $\delta=2$, is particularly simple and therefore well-suited for an analytical starting point. In binary state models, the attribute of agent $i$ at time $t$ is a binary variable $x_{i}(t) \in\{\boldsymbol{\square}, \square\} \equiv\{0,1\}$. The opinion profile is given by the bit-string $\mathbf{x}(t)=\left\{x_{1}(t), \ldots, x_{N}(t)\right\}$ so that, as for all binary single-step dynamics, the space of all possible configurations is the set of all bit-strings of length $N, \boldsymbol{\Sigma}=\{\mathbf{\square}, \square\}^{N}$.

For further convenience, let us use the convention that the black state is treated as zero and the white state as one $(\boldsymbol{\square} \equiv 0$ and $\square \equiv 1)$ and let us define the Hamming weight of a configuration as

$$
h(\mathbf{x})=\sum_{i=1}^{N} x_{i}=N_{\square}(\mathbf{x}) .
$$

Notice that the Hamming weight is precisely the measure usually considered in the analysis of binary opinion or population genetics models as it corresponds to the opinion or gene frequency. With $N_{\square}=h(\mathbf{x})$ and $N \mathbf{\square}=N-h(\mathbf{x})$, the Hamming weight $h(\mathbf{x})$ defines the most relevant macroscopic observable $\phi$ of interest in the context of these models.

As stated earlier, $h(\mathbf{x})$ (just as any other macroscopic observable) defines a partition of the configuration space $\boldsymbol{\Sigma}$. Namely, we can look at $h(\mathbf{x})$ as an equivalence relation such that any two configurations $\mathbf{x}, \mathbf{x}^{\prime} \in \boldsymbol{\Sigma}$ with $h(\mathbf{x})=h\left(\mathbf{x}^{\prime}\right)$ belong to the same equivalence class. The respective equivalence classes therefore collect all configurations with the same Hamming weight, or respectively, opinion frequency. Formally, let us define $X_{k} \subset \boldsymbol{\Sigma}$ by

$$
X_{k}=\{\mathbf{x}: h(\mathbf{x})=k\} .
$$

Each $X_{k} \in \mathbf{X}, k=0 \ldots N$ contains all the configurations $(\mathbf{x})$ in which exactly $k$ agents hold opinion $\square$ (and then $N-k$ hold opinion $\mathbf{\square}$ ). In this way we obtain a partition $\mathbf{X}=\left\{X_{0}, X_{1}, \ldots, X_{N}\right\}$ of the configuration space $\boldsymbol{\Sigma}$. Notice that $X_{0}$ and $X_{N}$ contain only one configuration, namely the final configurations $X_{0}=\{(\mathbf{\square} \ldots \mathbf{\square})\}$ and $X_{N}=\{(\square \square \ldots \square)\}$.

The projection of the VM micro chain $(\boldsymbol{\Sigma}, \hat{P})$ yields a new macro process with state space $\mathbf{X}=\left(X_{0}, \ldots, X_{N}\right)$. This is illustrated for a small system of three agents in Fig. 3.3. Noteworthy, in this macro description, the number of states is reduced from $2^{N}$ to $N+1$. While the number of states grows exponentially with the system size in the micro description, it grows now only linearly in the macro description.

Now, what are the conditions under which the macro process on the state space $\mathbf{X}=\left(X_{0}, \ldots, X_{N}\right)$ is again a Markov chain? It is easy to see that the partition $\mathbf{X}$ is preserved under the group of all permutations of the $N$ agents, denoted by $\mathcal{S}_{N}$. Agent permutations are also compatible with the equivalence relation defined by $h(\mathbf{x})$ because $h(\mathbf{x})$ is invariant with respect 


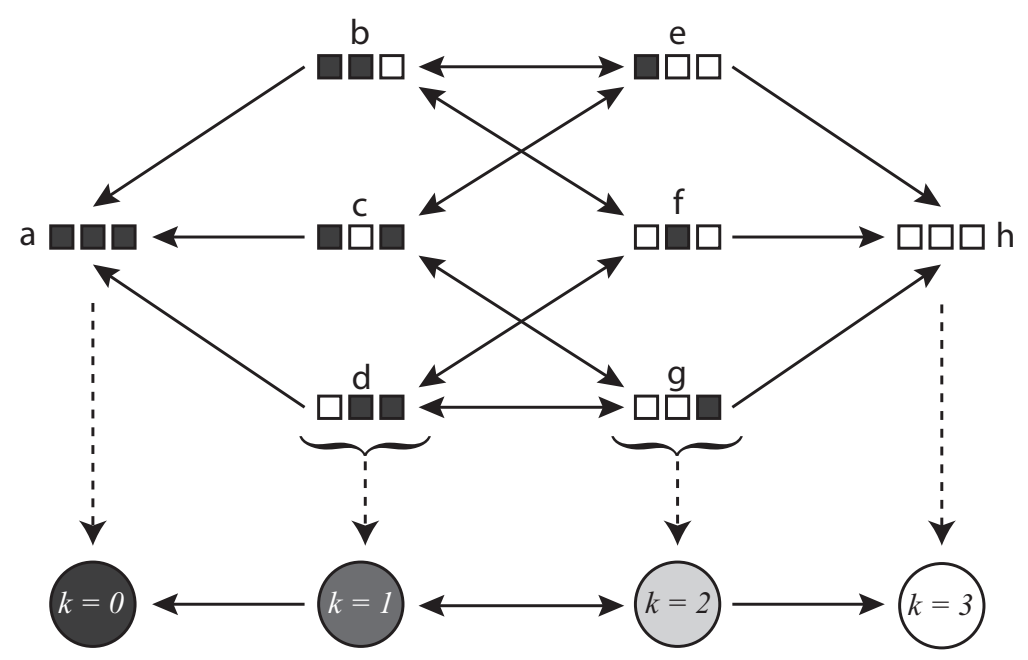

Figure 3.3: The micro chain for the VM with 3 agents and its projection onto a birth-death random walk obtained by agglomeration of states with the Hamming weight $h(\mathbf{x})=k$.

to any alternative labeling of the agents. We can therefore use $\mathcal{S}_{N}$ in the construction of a group of bijections $\mathcal{G}$ that satisfies Thm. 3.2.2. Namely, for each $\sigma \in \mathcal{S}_{N}$ we define a $\hat{\sigma} \in \mathcal{G}$ such that

$$
\hat{\sigma}(\mathbf{x}):=\left(x_{\sigma 1}, \ldots, x_{\sigma i}, \ldots, x_{\sigma N}\right) .
$$

The respective group $\mathcal{G}$ acting on $\boldsymbol{\Sigma}$ preserves $\mathbf{X}$ and is compatible with $h(\mathbf{x})$ : that is, for $\forall \hat{\sigma} \in \mathcal{G}$ and $\forall X_{i} \in \mathbf{X}, \mathbf{x} \in X_{i}$ implies that $\hat{\sigma}(\mathbf{x}) \in X_{i}$ and $h(\mathbf{x})=h(\hat{\sigma}(\mathbf{x}))$. Therefore, according to Thm. 3.2.2, lumpability leans on the condition of the invariance of the Markov transition matrix $\hat{P}$ under the permutation group of agents, $\hat{P}(\mathbf{x}, \mathbf{y})=\hat{P}(\hat{\sigma}(\mathbf{x}), \hat{\sigma}(\mathbf{y}))$. It is easy to see by Eq. (3.7) that this is satisfied if the probability distribution $\omega$ is invariant under the permutation group $\mathcal{S}_{N}$ and therefore uniform: $\omega(i, j)=1 / N^{2}$, for all pairs of agents $(i, j){ }^{1}$

This emphasizes the particular role of homogeneous mixing in the context of these models. Homogeneous mixing is special insofar as we can get rid of the sum in Eq. (3.7) because $\omega$ is uniform. In this case $\hat{P}(\mathbf{x}, \mathbf{y})$ can be expressed only in terms of the Hamming weight of $\mathbf{x}$

$$
\hat{P}(\mathbf{x}, \mathbf{y})=\sum_{x_{i} \neq x_{j}} \omega(i, j)=\frac{h(\mathbf{x})[N-h(\mathbf{x})]}{N^{2}},
$$

because only the numbers of possible attribute pairs with $\left(x_{i}, x_{j}\right)=(\square, \boldsymbol{\square})$ (respectively $\left(x_{i}, x_{j}\right)=(\boldsymbol{\square}, \square)$ ) matter. For the macro chain we obtain

\footnotetext{
${ }^{1}$ Notice that permutation invariance is also present if $»$ self interactions « are excluded such that $\omega(i, i)=0$. Then $\omega(i, j)=1 / N(N-1), \forall i \neq j$. For the following computations the possibility that an agent $i »$ interacts « with itself is not excluded.
} 
therefore:

$$
P\left(X_{k}, X_{k+1}\right)=P\left(X_{k}, X_{k-1}\right)=\frac{k(N-k)}{N^{2}} .
$$

and

$$
P\left(X_{k}, X_{k}\right)=\frac{k^{2}+(N-k)^{2}}{N^{2}} .
$$

The macro process specified by Eqs. (3.16) and (3.17) is a typical birthdeath random walk sometimes referred to as Moran process (Moran, 1958). It has two absorbing states $X_{0}$ and $X_{N}$ for $P\left(X_{0}, X_{0}\right)=P\left(X_{N}, X_{N}\right)=1$ corresponding to the two consensus configurations with all agents in the same state. The Markovianity of the VM process obtained by a projection onto $\mathbf{X}=\left(X_{0}, \ldots, X_{N}\right)$ is well-established in the case of homogeneous mixing, but it seems to be less well-known that the preservation of Markovianity by this projection construction is the exception rather than the rule. This is due to the fact that for heterogeneous $\omega$ the second equality in Eq. (3.15) does not hold in general, and the transition rates depend not only on the Hamming weight, but also on the population structure.

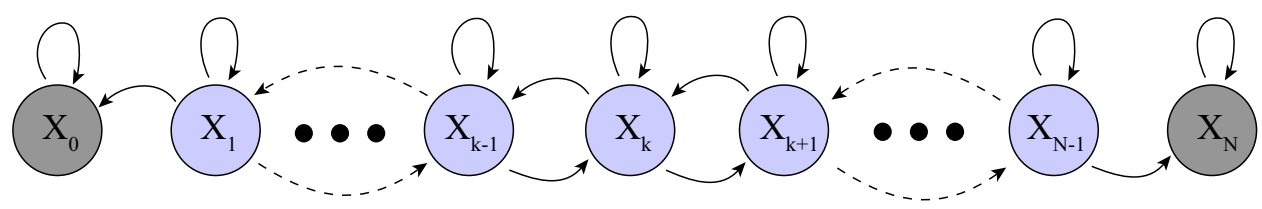

Figure 3.4: Macro chain for the binary VM with homogeneous mixing.

The macro chain for the binary VM with homogeneous mixing is shown in Fig. 3.4. The transition probability matrix $P$ of the Markov chain is given by the stochastic transition matrix:

$$
P=\left(\begin{array}{ccccccc}
1 & 0 & 0 & 0 & 0 & \ldots & 0 \\
p_{1} & q_{1} & p_{1} & 0 & 0 & \ldots & 0 \\
0 & p_{2} & q_{2} & p_{2} & 0 & \ldots & 0 \\
\vdots & \ddots & \ddots & \ddots & & & \vdots \\
0 & & p_{k} & q_{k} & p_{k} & & 0 \\
\vdots & & \ddots & \ddots & \ddots & & \vdots \\
0 & \ldots & 0 & p_{N-2} & q_{N-2} & p_{N-2} & 0 \\
0 & \ldots & 0 & 0 & p_{N-1} & q_{N-1} & p_{N-1} \\
0 & \ldots & 0 & 0 & 0 & 0 & 1
\end{array}\right)
$$

with $p_{k}=P\left(X_{k}, X_{k \pm 1}\right)$ and $q_{k}=P\left(X_{k}, X_{k}\right)$ given in (3.16) and (3.17). 
The probability that any opinion change happens in the system is $2 p_{k}$ and then depends on the current opinion balance. But there is no general tendency of the system to be attracted by one of the extremes. In other words, the macro chain performs an unbiased random walk. Due to the particular form of $p_{k}$ the prevalence of one opinion results in a reduced probability of further opinion change, contrary to the usual random walk with constant transition probabilities.

For $k \cong \frac{N}{2}$ we have $p_{k} \cong \frac{1}{4}$. By contrast, when $k$ is closed to 0 or $N$, there is a large probability for the system to stay unchanged. Notice that for $k=1$ or $k=N-1$ this probability tends to 1 when $N \rightarrow \infty$. This indicates that in this model once one opinion dominates over the other, public opinion as a whole becomes less dynamic, which also reveals a difficulty for new opinions to spread in the artificial society.

\subsubsection{Transient Macro Dynamics}

In Markov chains with absorbing states (and therefore in ABMs) the asymptotic status is quite trivial. As a result, it is the understanding of the transient that becomes the interesting issue. We shall now analyze the transient dynamics for the macro dynamics of the binary VM. In order to do so, all that is needed is to compute the fundamental matrix $\mathbf{F}$ of the Markov chain (Kemeny and Snell, 1976; Behrends, 2000).

Let us express $P$ in its standard form in which the two first rows and columns stand for the absorbing states $X_{0}$ and $X_{N}$ and the remaining for the $N-1$ transient states:

$$
P=\left(\begin{array}{l|l}
1 & 0 \\
\hline R & Q
\end{array}\right)
$$

Here, $Q$ is the $(N-1) \times(N-1)$ matrix corresponding to the transient states (without the first two rows and columns associated with $X_{0}$ and $X_{N}$ ). The fundamental matrix $F$ is the inverse of $(\mathbf{1}-Q)$ where $\mathbf{1}$ is the $(N-1) \times(N-1)$ identity matrix. Due to the structure of $P,(\mathbf{1}-Q)$ is a tridiagonal matrix that can be inverted using, for instance, the tridiagonal matrix algorithm (also known as Thomas algorithm, Conte and Boor, 1980).

For the VM, moreover, we have $P\left(X_{k}, X_{k+1}\right)=P\left(X_{k}, X_{k-1}\right)=p_{k}$ which allows for an analytical inversion of $(\mathbf{1}-Q)$. We have

$$
(\mathbf{1}-Q)=\left(\begin{array}{ccccccc}
2 p_{1} & -p_{1} & 0 & 0 & 0 & \ldots & 0 \\
-p_{2} & 2 p_{2} & -p_{2} & 0 & 0 & \ldots & 0 \\
\vdots & \ddots & \ddots & \ddots & & & \vdots \\
0 & & -p_{k} & 2 p_{k} & -p_{k} & & 0 \\
\vdots & & \ddots & \ddots & \ddots & & \vdots \\
0 & \ldots & 0 & 0 & -p_{N-2} & 2 p_{N-2} & -p_{N-2} \\
0 & \ldots & 0 & 0 & 0 & p_{N-1} & 2 p_{N-1}
\end{array}\right) .
$$


In order to compute $(\mathbf{1}-Q)^{-1}$ we can use the system of equations defined by $\mathbf{1}=(\mathbf{1}-Q)^{-1} \mathbf{F}$ and $\mathbf{1}=\mathbf{F}(\mathbf{1}-Q)^{-1}$. Due to the fact that there is only one variable per row (namely, $p_{k}$ ) the recursive equations that have to be solved simplify and we have as a general solution of $(\mathbf{1}-Q)^{-1}$ :

$$
F_{i j}=\left\{\begin{array}{ll}
\frac{i(N-j)}{N p_{j}} & : i \leq j \\
\frac{j(N-i)}{N p_{j}} & : i>j
\end{array}\right\} .
$$

For the VM, with $p_{k}$ given by Eq. (3.16) we obtain:

$$
F_{i j}=\left\{\begin{array}{cc}
\frac{N i}{j} & : i \leq j \\
\frac{N(N-i)}{N-j} & : i>j
\end{array}\right\} .
$$

Eq. (3.22) provides us with the fundamental matrix of the system for an arbitrary number of agents $N$, giving information about mean quantities of the transient dynamics in this model.

The corresponding matrix $\mathbf{G}$ that encodes information about the variance (Kemeny and Snell, 1976, 82-84) of the same quantities can be computed on the basis of $\mathbf{F}$ by

$$
G=\mathbf{F}\left(\mathbf{F}_{\text {diag }}-\mathbf{1}\right) \mathbf{F}_{\text {square }}
$$

where $\mathbf{F}_{\text {diag }}$ contains the diagonal elements of $\mathbf{F}$ and is zero for the nondiagonal elements and $\left(\mathbf{F}_{\text {square }}\right)_{i j}=(\mathbf{F})_{i j}^{2}$. For the VM it reads:

$$
G_{i j}=\left\{\begin{array}{cc}
N(N-1) & : i=j \\
\left(2 N^{2}-N\right) \frac{(N-i)}{(N-j)}-N^{2} \frac{(N-i)^{2}}{(N-j)^{2}} & : i>j \\
\left(2 N^{2}-N\right) \frac{i}{j}-N^{2} \frac{i^{2}}{j^{2}} & : i<j
\end{array}\right\} .
$$

The matrices $\mathbf{F}$ and $\mathbf{G}$ provide us with a good understanding about the transient dynamics of the VM: $F_{i, k}$ is the mean of the time the process is in the transient configuration $X_{k}$ when started in the configuration $X_{i}$ and $G_{i, k}$ is the corresponding variance.

An interesting quantity to characterize opinion dynamics is the time a process starting in $X_{k}$ takes to end in one of the two consensual absorbing states. Defining $\tau_{k}$ and $v_{k}$ as the mean and the variance of the random variable for $k=1, \ldots, N-1$ we got from (3.22) and Kemeny and Snell (1976):

$$
\tau_{k}=N\left[\sum_{j=1}^{k-1} \frac{(N-k)}{(N-j)}+1+\sum_{j=k+1}^{N-1} \frac{k}{j}\right]
$$

and the corresponding expression for $v$ can explicitly be written from (3.24) using:

$$
v=(2 \mathbf{F}-\mathbf{1}) \tau-\tau_{s q}
$$


where $\tau_{s q}$ denotes the vector resulting from $\tau$ by squaring each entry. This yields

$$
\begin{array}{r}
v_{k}=2 N^{2}(N-k)\left[\sum_{i=1}^{k-1} \frac{1}{(N-i)}\left(\sum_{j=1}^{i-1} \frac{(N-i)}{(N-j)}+1+\sum_{j=i+1}^{N-1} \frac{i}{j}\right)\right]+ \\
+(2 N-1) N\left(\sum_{j=1}^{k-1} \frac{(N-k)}{(N-j)}+1+\sum_{j=k+1}^{N-1} \frac{k}{j}\right)+ \\
+2 N^{2} k\left[\sum_{i=1}^{N-1} \frac{1}{k+i}\left(\sum_{j=1}^{k+i-1} \frac{(N-k-i)}{(N-j)}+1+\sum_{j=k+i+1}^{N-1} \frac{k+i}{j}\right)\right]- \\
-N^{2}\left(\sum_{j=1}^{k-1} \frac{(N-k)}{N-j}+1+\sum_{j=k+1}^{N-1} \frac{k}{j}\right)^{2} .
\end{array}
$$

For a system of 1000 agents, Fig. 3.5 shows the mean times until absorption $\tau_{k}$ from each $X_{k}$ and the corresponding variances $v_{k}$. Notice the contrast among the two scales showing how the variance is large compared with the mean.
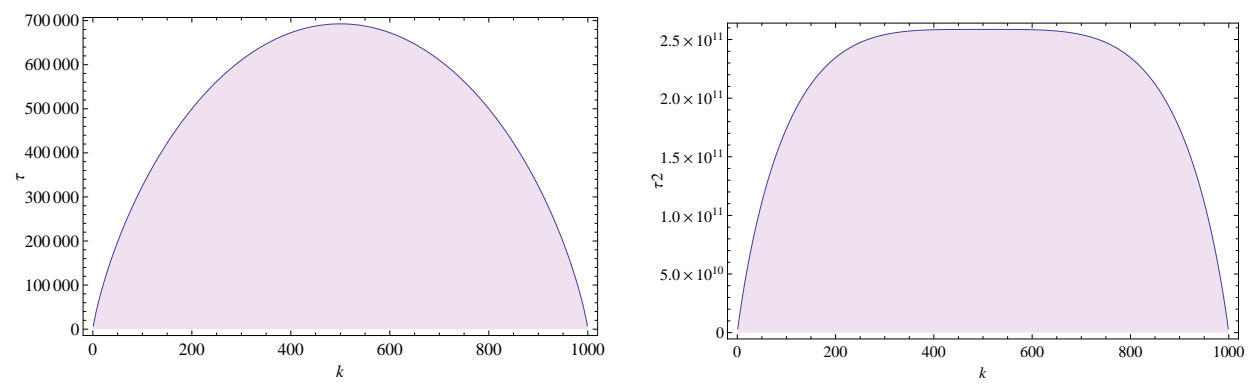

Figure 3.5: Mean time $\tau_{k}$ (l.h.s.) and variance $v_{k}$ (r.h.s.) until absorption as a function of the initial configuration $X_{k}$ for $N=1000$.

There are interesting consequences of (3.25) and (3.27), in cases where the number of agents $(N)$ becomes large. First, as already pointed out, we see that the ratio between the variance and the mean is quite large and in fact it diverges with $N$. Hence, the means are fairly unreliable estimates in this system. This is often the case for absorbing Markov chains (Kemeny and Snell, 1976) making a direct interpretation of numerical simulations for this type of models tough. Even more subtle, the time scale depends significantly on the starting configuration $k$. In fact $\tau_{k}$ scales as $N \log N$ for $k=1$ and $k=N-1$ but as $N^{2}$ for $k=\frac{N}{2}$. We are therefore faced with a situation where to take the limit of asymptotic times first and then large number of agents or to do it in the reverse order is not equivalent. In other words, for a 
finite, even large, number of agents, there is a probability 1 of reaching one of the consensual configurations in finite time. By contrast, in the limit of an infinite number of agents this probability is 0 and the process will stay essentially in the configurations close to parity, $k=\frac{N}{2}$. Together with the presence of large fluctuations revealed in (3.27) (see Fig. 3.5) this fact is the imprint of a (dynamical) phase transition.

Besides this analysis of the scaling law of the dynamics for large $N$, it is also interesting to have an insight into the distributions of absorbing times for a system of fixed number of agents, the second item mentioned above. As known by the Perron-Frobenius Theorem (Seneta, 2006) this distribution is exponential for large $t$ with rate $\left(1-\lambda_{\max }\right), \lambda_{\max }$ being the maximal eigenvalue of the matrix $Q$. However, the correction to this distribution for intermediate times depends on the initial configuration. Indeed in our case, the distribution of the times taken by the process to fall into one of the consensual configurations departs from the exponential in a way that is strongly dependent upon the initial state, as shown in Figs. 3.6 and 3.7.

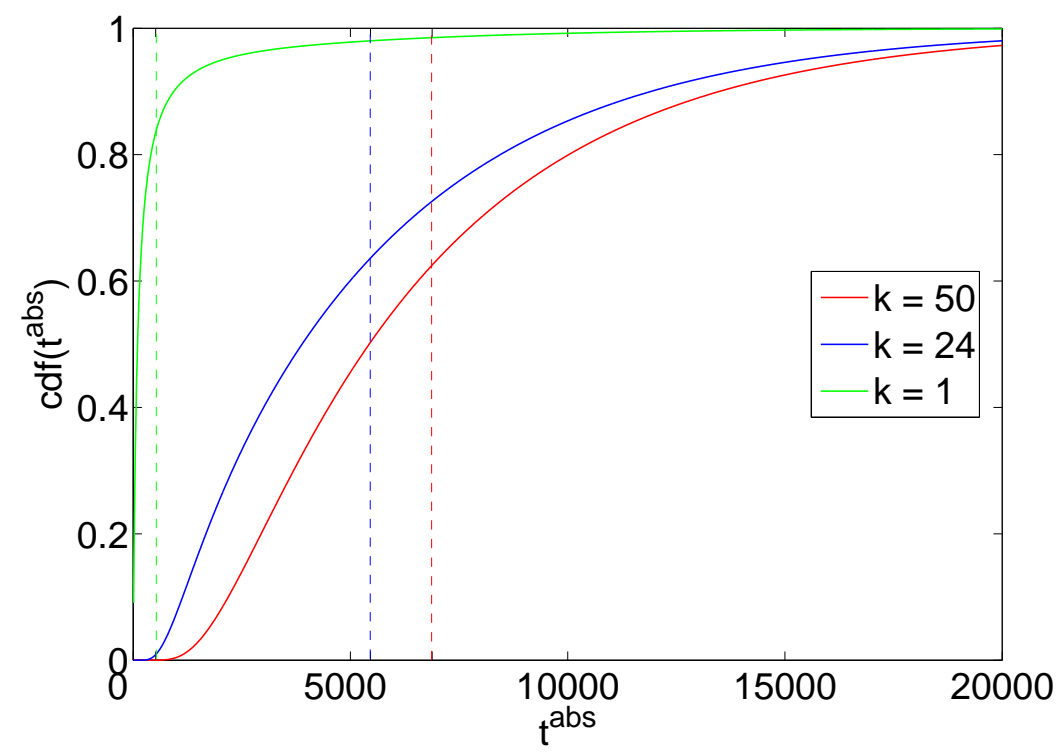

Figure 3.6: Cumulative probability of being absorbed after time $t^{a b s}$ for $N=100$ and three starting configurations $k=1$ (green), $k=24$ (blue) and $k=50$ (red). Vertical lines show the respective expected mean convergence times $\tau_{k}$.

The computation of the full time distribution is based on the fact that the powers $Q^{t}$ of $Q$ contain all the information about the probability that the process is still not absorbed after $t$ steps. To be precise, the sum over the $k$ th row of $Q^{t}$ equals the probability that the process starting at $X_{k}$ is not absorbed after $t$ iterations. This yields the cumulative distribution function 


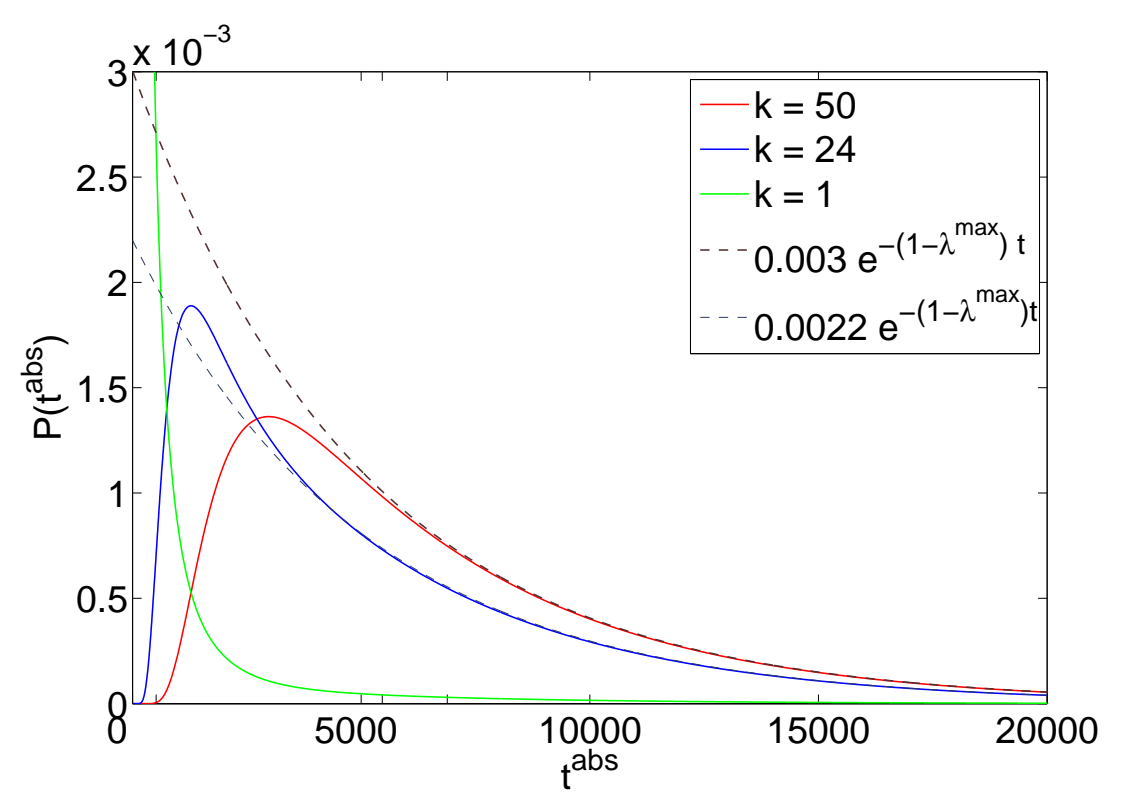

Figure 3.7: Probability of absorbency at time $t^{a b s}$ for $N=100$ and three starting configurations $k=1$ (green), $k=24$ (blue) and $k=50$ (red). Exponential functions (dashed) are shown to illustrate the exponential decay of the convergence times as a function of $\left(1-\lambda_{\max }\right)$.

shown in Fig. 3.6 for a system of 100 agents and three starting configurations $k=1$ (green), $k=24$ (blue) and $k=50$ (red). The vertical dashed lines represent the respective mean values $\tau_{k}$ obtained using Eq. (3.25). For $k=50$ it becomes clear that around $60 \%$ of simulation runs are absorbed until the expected absorption time is reached. Fig. 3.7 shows the probability that the process is absorbed exactly at time $t^{a b s}$. The three solid curves represent the respective probabilities for $k=1,24,50$. The dashed curves are exponential functions that fit the distributions for large $t^{a b s}$ showing that the distributions decay with $\left(1-\lambda_{\max }\right)$ as claimed above.

This leads to an interesting feature of the distribution of the absorption times coming from the fact that $\lambda_{\max }$ tends to one when $N \rightarrow \infty$. More precisely (Seneta, 2006) and (3.18) implies

$$
1>\lambda_{\max } \geq 1-p_{1} \geq \frac{N-1}{N} .
$$

As a consequence, we see that the times for the system to get absorbed in the final states diverge with $N$, and $Q$ approaches a stochastic matrix. In fact in the limit of infinite $N$ consensus cannot be reached. This is not the only reason why the dynamics inside the transient configurations is so important. In fact we might speculate that, in a more realistic description, exogenous events may interfere with the system and reset it from time to time, and 
then, in view of the previous analysis, even when the number of agents is finite but sufficiently large, the system will similarly never fall into a final absorbing consensus configuration.

Notice that Eqs. (3.22) and (3.24) can be used to gain new insight into the dynamics inside the transient. As noted above, $F_{i, k}$ is the mean of the time the process is in the transient configuration $X_{k}$ when started in the configuration $X_{i}$ and $G_{i, k}$ is the corresponding variance. Figs. 3.8 and 3.9 show a quite different behavior depending on the initial situation. Starting from $X_{i}$ close to $X_{1}$ or $X_{N-1}$ - the strongly »biased « configurations - the residence mean times in $X_{k}$ naturally decrease with the distance from $i$, but become almost independent of $k$ and $N$ for large $k$, whereas the corresponding variance diverges with $N$. Instead, starting from $X_{i}$ close to $X_{N / 2}$ - the quasi-homogeneous configurations - the residence mean times and variance in $X_{k}$ always diverge.
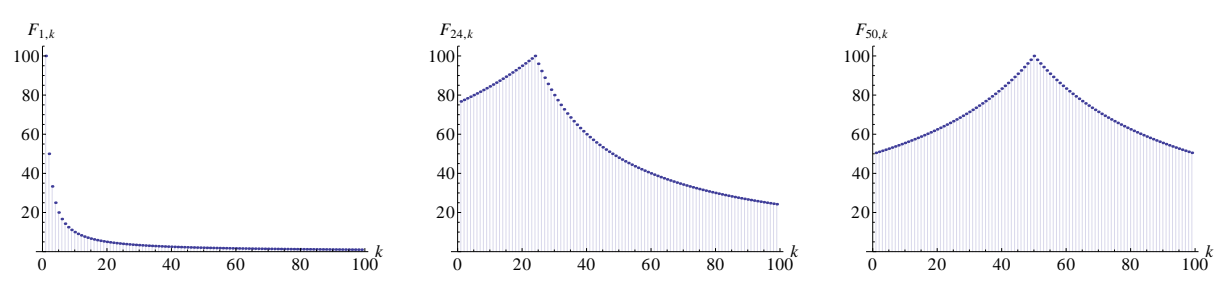

Figure 3.8: The mean times for the process in a configuration $X_{k}$ before absorption for a walk starting in $X_{1}, X_{24}$ and $X_{50}$ as function of $k$ for $N=$ 100.
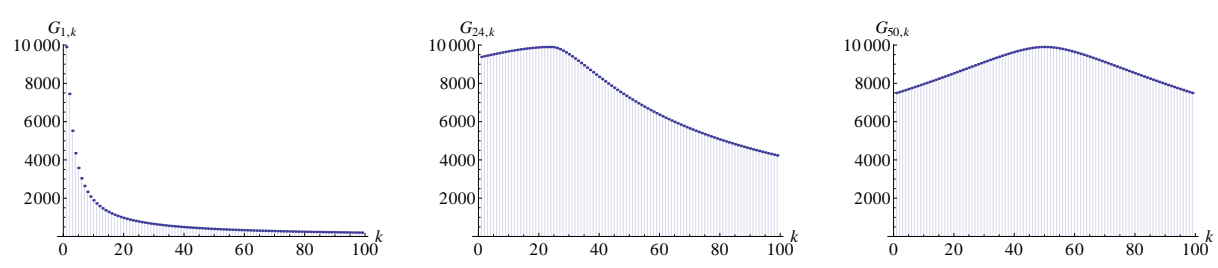

Figure 3.9: The variance in the number of times a realization starting in $X_{1}, X_{24}$ and $X_{50}$ is in $X_{k}$ before absorption as function of $k$ for $N=100$. Notice the scale as compared with Fig. 3.8

The reason for such »strange « behavior is quite clear: as $N$ becomes large, almost all the realizations are trapped during very large times close to their initial configuration, see (3.18), and only very few realizations reach the opposite configurations but staying there for large times. That is, a complete overturn of the opinions is very rare but, when happened, the new situation naturally becomes as stable as the previous. Therefore we are in a case where there is almost no realization behaving as the mean. On the other hand, starting from $X_{i}$ closed to $X_{N / 2}$, the »homogeneous" 
configurations, the mean times in $X_{k}$ also decrease with the distance from $N / 2$, but now the mean times all scale linearly with $N$ and the variances with $N^{2}$. Surprisingly these two behaviors - almost static on the border and very unstable »back-and-forth « in the center - compensate perfectly to end up in the same mean residence times and variance (the diagonals of $\mathbf{F}$ and G) for all the initial configurations. The same compensation appears when we compare the probabilities for a walk stating in $X_{i}$ to return in $X_{i}$, which is independent of $i$ and almost sure for large $N$ :

$$
\lim _{t \rightarrow \infty} P^{(t)}\left(X_{i}, X_{i}\right)=\frac{F_{i i}-1}{F_{i i}}=\frac{N-1}{N} .
$$

It is reasonable to hypothesize a correlation, if not a causal link, between fast changes in the agent opinion induced by the social process, here stylized in the dynamical rules, and the inconsistency experienced by agents between the micro and the macro level. This conflict is referred to as practical emergence (Giesen, 1987). It consists of a gradual separation of the individual mental patterns from the reality. The agent is then faced with a representation that is not always perfectly in keeping with the situation (Giesen, 1987, 342). In the opinion model, a possible rating of this practical emergence inconsistency is the mean time the macro process takes to change of state. Indeed any change of state in this process corresponds to an opinion change of an agent. Therefore the faster this rate, the smaller the switching mean time, and the more likely is the emergence of a practical disruption between picture and reality from the agent's point of view.

From (3.16) and Kemeny and Snell (1976), Thm. 3.5.6, the mean time $\eta_{k}$ that the process remains in state $X_{k}$ once the state is entered (including the entering step) is:

$$
\eta_{k}=\frac{N^{2}}{2 k(N-k)}
$$

Therefore, $\eta_{k}$ is of order $\frac{N}{2}$ for $k$ close to (but smaller than) $N$ and 2 for $k$ close to $\frac{N}{2}$. Again, for $N$ large the process will be almost stationary in presence of a large majority supporting one of the opinions but extremely unstable when no opinion is clearly predominant. In the latter case practical emergence is plausible. We suggest correlating small values of $\eta_{k}$ with this phenomenon.

To conclude the analysis of the transient dynamics, Fig. 3.10 shows different realizations of the agent simulation along with the expected evolution in form of a confidence interval. The measure of the realizations inside a given confidence interval is an increasing function of time. However, since any individual realization may cross the border of this interval several times before falling in one of the final absorbing states a numerical evaluation of the convergence times may be quite delicate. 


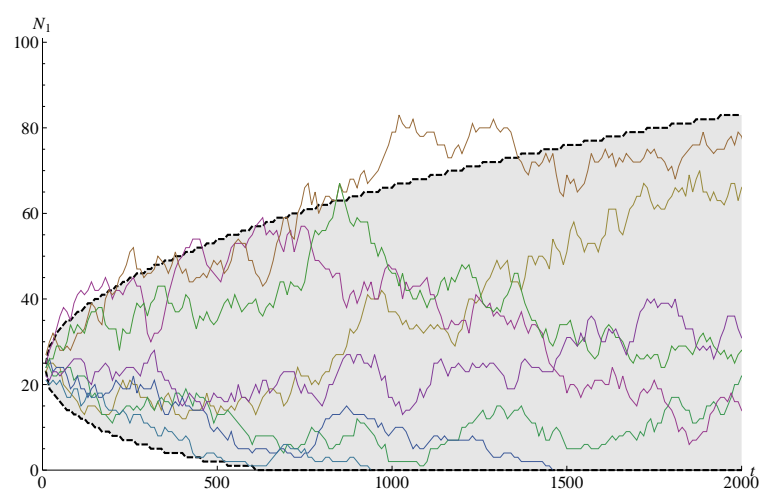

Figure 3.10: Different realizations of simulations with 24 out of 100 agents in initial state $\square$ (i.e a process starting in $X_{24}$ ). Markov chain analysis shows that with probability 0.95 the process is in the shaded region.

\subsubsection{Exit Probabilities}

The fundamental matrix F, Eq. (3.22), can also be used to compute the probabilities for a process starting in $X_{i}$ to end up in $X_{0}$ or $X_{N}$. These probabilities are obtained by multiplying $\mathbf{F} R$ where $R$ is the respective submatrix from the canonical form of $P$ shown in Eq. (3.19). It is well-known that the exit probabilities depend linearly on the initial proportion of agents in the different states as

$$
\lim _{t \rightarrow \infty} P^{(t)}\left(X_{i}, X_{0}\right)=\frac{N-i}{N}
$$

and

$$
\lim _{t \rightarrow \infty} P^{(t)}\left(X_{i}, X_{N}\right)=\frac{i}{N}
$$

In other words, the probability to end up in configuration $(\square \square \ldots \square)$ is proportional to the initial number of $\square$-agents.

\subsubsection{Macrodynamics of the General Voter Model}

For the VM with $\delta$ different attributes, the state of any agent $i$ at time $t$ is a variable $x_{i}(t) \in\{0, \ldots, \delta-1\}$. The opinion profile is given by the vector $\mathbf{x}(t)=\left\{x_{1}(t), \ldots, x_{N}(t)\right\}$. The space of all possible configurations is then $\boldsymbol{\Sigma}=\{0, \ldots, \delta-1\}^{N}$. As described in Sec. 3.1, the micro chain of singlestep model with $|\mathbf{S}|=\delta$ is a random walk with loops on the Hamming graph $H(N, \delta)$.

In the projection construction, we follow the same argument as for $\delta=2$. We define $N_{s}(\mathbf{x})$ to be the number of agents in the configuration $\mathbf{x}$ with 
opinion $s, s=0, \ldots, \delta-1$, and then $X_{\left\langle k_{0}, k_{1}, \ldots, k_{\delta-1}\right\rangle} \subset \boldsymbol{\Sigma}$ as

$$
\begin{array}{r}
X_{\left\langle k_{0}, \ldots, k_{s}, \ldots, k_{\delta-1}\right\rangle}=\left\{\mathbf{x} \in \mathbf{\Sigma}: N_{0}(\mathbf{x})=k_{0}, \ldots, N_{s}(\mathbf{x})=k_{s}, \ldots\right. \\
\left.\ldots, N_{\delta-1}(\mathbf{x})=k_{\delta-1} \text { and } \sum_{s=0}^{\delta-1} k_{s}=N\right\} .
\end{array}
$$

Each $X_{\left\langle k_{0}, k_{1}, \ldots, k_{\delta-1}\right\rangle}$ contains all the configurations $\mathbf{x}$ in which exactly $k_{s}$ agents hold opinion $s$ for any $s$. We use the notation $\left\langle k_{0}, k_{1}, \ldots, k_{\delta-1}\right\rangle$ to indicate that $\sum_{s=0}^{\delta-1} k_{s}=N$. As in the binary case, we obtain in this way a partition $\mathbf{X}$ of the configuration space $\boldsymbol{\Sigma}$.

Again, as for the binary model, the symmetry condition (3.8) of Thm. 3.2 .2 is verified if the probability distribution $\omega$ is permutation invariant and therefore uniform: $\omega(i, j)=\frac{1}{N^{2}}$, for all pairs of agents $(i, j)$. That is, the projection of the micro process $(\boldsymbol{\Sigma}, \hat{P})$ onto $\mathbf{X}$ yields a Markov chain in the case of homogeneous mixing. In this case, Eq. (3.16) generalizes to:

$$
P\left(X_{\left\langle k_{0}, k_{1}, \ldots, k_{\delta-1}\right\rangle}, X_{\left\langle k_{0}^{\prime}, k_{1}^{\prime}, \ldots, k_{\delta-1}^{\prime}\right\rangle}\right)=\frac{k_{s} k_{r}}{N^{2}}
$$

if $k_{s}^{\prime}=k_{s} \pm 1$ and $k_{r}^{\prime}=k_{r} \mp 1$ whereas $k_{j}^{\prime}=k_{j}$ for all other $j$, and the probability that no opinion changes, Eq. (3.17), becomes

$$
P\left(X_{\left\langle k_{0}, k_{1}, \ldots, k_{\delta-1}\right\rangle}, X_{\left\langle k_{0}, k_{1}, \ldots, k_{\delta-1}\right\rangle}\right)=\frac{1}{N^{2}} \sum_{s=0}^{\delta-1}\left(k_{s}\right)^{2} .
$$

The structure of (3.34) has an interesting consequence on the dynamics of the system. We see that, if there is an $s$ for which $k_{s}=0$, the probability of transition to a state with $k_{s}>0$ is zero. In other words, to change the number of agents sharing opinion $s$, at least one agent with such an opinion is needed. Therefore, the state space is organized as a $\delta$-simplex with absorbing faces ordered by inclusion, corresponding to increasing sets of opinions with no supporters.

Starting in some state with no null $k_{s}$ the process will finish at certain time in a state where, for the first time, $k_{s}=0$ for some $s$ (notice that only one $s$ at each time can fall to zero since the sum of all $k_{s}$ is constant). From there, the given $k_{s}$ will stay equal to zero for ever, and $(3.34-3.35)$ tell us that the transition probabilities are now those of a system with $\delta-$ 1 opinions. Because the condition $\sum_{s=0}^{\delta-1} k_{s}=N$ is to be fulfilled by the remaining opinions, the system will then evolve exactly as if the $N$ agents share $\delta-1$ opinions from the very beginning. After a certain time a new opinion will lose all its supporters and the system is now equivalent to a full system of $\delta-2$ opinions, and so on. The system will cascade up to the final 
absorbing state, with only one opinion shared by all the $N$ agents. We recall that each of such cascade transitions is achieved in finite (random) times.

By computing the fundamental matrix of the subsystems it would be possible to access the mean and variance of the times the system evolves between two successive extinctions of group opinions. We conjecture the same scaling laws for a system of $\delta$ opinions as the ones already described for $\delta=2$.

\subsubsection{Further Reduction}

Alternatively, we can make use of the symmetries in the structure of (3.34) and search for lumpable partitions to further reduce the problem. This can be done by considering the model from the perspective of a single »party « associated with (say) opinion 0 . For that $»$ party «, it may be important to know how many agents are supportive because they share the same opinion, and how many are not because they support one of the remaining opinions. Thus, we reduce the model to a quasi-binary variant with the supporter opinion 0 on one side and all other opinions $(1 \cup \cdots \cup \delta-1)$ on the other, grouping together all the states with $k_{0}=r, r=0, \ldots, N$.

The corresponding partition reads:

$$
Y_{r}^{0}=\bigcup_{\substack{k_{1}, \ldots, k_{\delta-1} \\ k_{0}=r}} X_{\left\langle r, k_{1}, \ldots, k_{\delta-1}\right\rangle}, r=0, \ldots, N .
$$

It is easy to verify that the chain (on $\mathbf{X}$ ) is indeed lumpable with respect to $\mathbf{Y}$ by considering the transition probabilities $(3.34){ }^{2}$ One can show that

$$
\sum_{\substack{k_{1}, \ldots, k_{\delta-1} \\ k_{0}=r}} P\left(X_{\left\langle r, k_{1}, \ldots, k_{\delta-1}\right\rangle}, Y_{r \pm 1}^{0}\right)=\frac{r(N-r)}{N^{2}}
$$

and therefore independent of the $k_{s}, s>0$. This tells us that

$$
P\left(Y_{r}^{0}, Y_{r+1}^{0}\right)=P\left(Y_{r}^{0}, Y_{r-1}^{0}\right)=\frac{r(N-r)}{N^{2}} .
$$

It thus turns out that the chain formed by the $Y_{r}^{0}, r=0,1, \ldots, N$ is exactly the same as the chain derived for the binary model. Therefore, the questions regarding the evolution of one opinion in relation to all the others taken together are addressed by the transient analysis performed in Sec. 3.3.2. That

\footnotetext{
${ }^{2}$ Alternatively, one could also verify the lumpability of $\mathbf{Y}$ directly with respect to the micro process. Namely, as shown in Sec. 3.1, the micro chain is a random walk on $H(N, \delta)$. The group $\mathcal{S}_{N}$ acting on the agents as well as the permutation group $\mathcal{S}_{\delta}$ acting on the agent attributes give rise to automorphisms of $H(N, \delta)$ such that the automorphism group is given by the direct product $\operatorname{Aut}(H(N, \delta))=\mathcal{S}_{N} \otimes \mathcal{S}_{\delta}$. The transformation group that generates the new partition $\mathbf{Y}$ is a subgroup of that, namely, $\Lambda=\mathcal{S}_{N} \otimes \mathcal{S}_{\delta-1} \subset \mathcal{S}_{N} \otimes \mathcal{S}_{\delta}$.
} 
is to say, from this point of view, each »party « may rely on the dynamics of a binary model as a coarse description of the evolution of its own status.

There is however an important subtlety when doing such an analysis. The asymmetry of the partition one-against-all-others will be encoded in the initial condition. For instance, starting with an equally distributed profile of $\mathrm{N}$ agents corresponds to the initial condition $X_{\langle k, k, \ldots, k\rangle}$ in the detailed description but to $Y_{N / \delta}^{0}$ in the coarse case. In such a way the asymmetry in the one-against-all-others description is recovered.

In particular, this tells us that the probability to end up in the final configuration $Y_{N}^{0}=\{(\boldsymbol{\square} \ldots \boldsymbol{\square})\}$ is proportional to this initial bias and becomes

$$
\lim _{t \rightarrow \infty} P^{(t)}\left(Y_{N / \delta}^{0}, Y_{N}^{0}\right)=\frac{1}{\delta}
$$

Consequently, with probability $1-1 / \delta$ the process will transit to the class of states in which $r=0$ with a zero probability to return to the $r>0$ class. Viewed in the space $\mathbf{X}$, the process is not finished then, but performs a random walk until one of the uniformity states is reached, each with equal probability $1 / \delta$.

\subsection{Bounded Confidence and the Emergence of Opin- ion Polarization}

An important issue in the study of opinion dynamics concerns the effects of bounded confidence on the model dynamics. Especially the models conceived by Hegselmann and Krause (2002) and Deffuant et al. (2001) (but also Axelrod, 1997; Banisch et al., 2010) are designed to study the situation that the willingness of agents to communicate depends on the similarity of their attributes. It is also noteworthy that similarity constraints of this kind play an important role in population genetics, where they go under the label assortative mating (e.g., Kondrashov and Shpak, 1998; Dieckmann and Doebeli, 1999 see also Banisch and Araújo, 2012).

In this section, we treat in detail the simplest case where bounded confidence (and other communication constraints) can be integrated, namely $\delta=3$. Consider that agents can choose between three different alternatives $\mathbf{S}=\{a, b, c\}$. In order to model bounded confidence we define a $\mathbf{S} \times \mathbf{S} \gg$ confidence matrix « $\alpha$ which encodes for any attribute pair whether or not the attributes are compatible. If all entries in $\alpha$ are one, this yields the unconstrained VM with $\delta=3$ and the results of the previous section apply. For bounded confidence, we set $\alpha(a, c)=\alpha(c, a)=0$ meaning that the attributes $a$ and $c$ are incompatible $(a \leftrightarrow c)$. The consequence of this constraint is the emergence of non-consensual absorbing states, that is, the stable co-existence of different attributes. 


\subsubsection{The Unconstrained Case}

We are particularly interested in the $\delta=3$ case because it is the simplest version in which one can meaningfully consider the effects of bounded confidence. According to the general results of Sec. 3.3.4, the projection from micro to macro dynamics is lumpable with respect to $\mathbf{X}$ (under the homogeneous hypothesis on $\omega$ of course). We denote the number of $a, b$ and $c$ agents by (respectively) $k, l$ and $m$ so that $\mathbf{X}=\left\{X_{\langle k, l, m\rangle}: 0 \leq k, l, m \leq\right.$ $N, k+l+m=N\}$. The Markov chain topology obtained by this projection is shown in Fig. 3.11 along with the transition structure for a system of 8 agents. The probabilities of the transitions are given by Eqs. (3.34) and (3.35) which allows us to compute the complete transition matrix $P$.

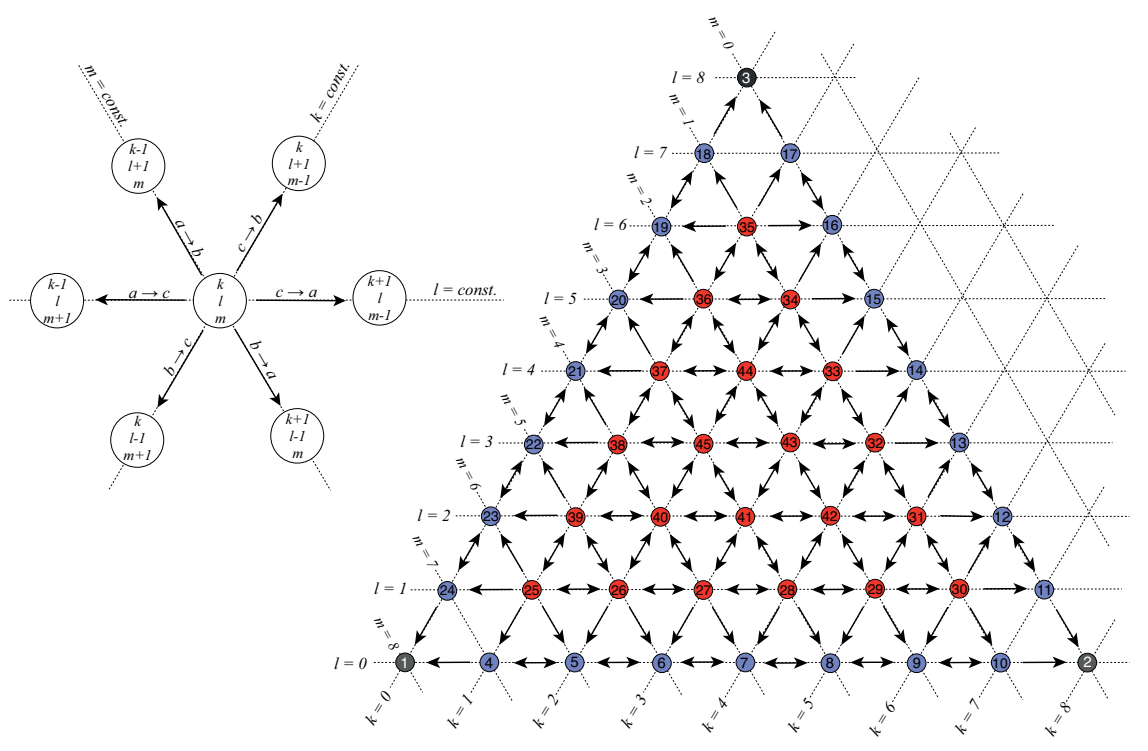

Figure 3.11: Transition structure (1.h.s) and state topology (r.h.s) of the unbounded confidence model with three opinions $\mathbf{S}=\{a, b, c\}$, here $N=8$.

For the construction of $P$, the nodes in the Markov chain are labeled in increasing order from the absorbing to the central nodes, see Fig. 3.11: labels 1 to 3 (black) for absorbing consensus states, labels 4 to 24 (blue) for two-opinion states, labels 25 to 39 (red) for three-opinion states with one of the opinion supporters reduced to one element, and labels 40 to 45 (red) for the remainder states. It is possible to compute the fundamental matrix, at least numerically if $N$ is large, and this makes it possible to compute the significant statistical indicators of the model. For instance, if $N=8$, the state space of the macro dynamics has 45 states and the mean times for the transient nodes to reach an absorbing state (consensus) range between 21 and 48 time steps, see Fig. 3.15. Not surprisingly the mean transition times are a function of the distance to the absorbing states as measured on the 
graph of the state space (Fig. 3.11).

From the fundamental matrix $\mathbf{F}$ it is also easy to compute the probabilities of ending up in each of the absorbing (consensus) states starting from any transient node using the matrix $B=\mathbf{F} R$, where $R$ is defined as in (3.19). For instance, for $N=8$, the absorbing probabilities for any state are represented in Fig. 3.12.

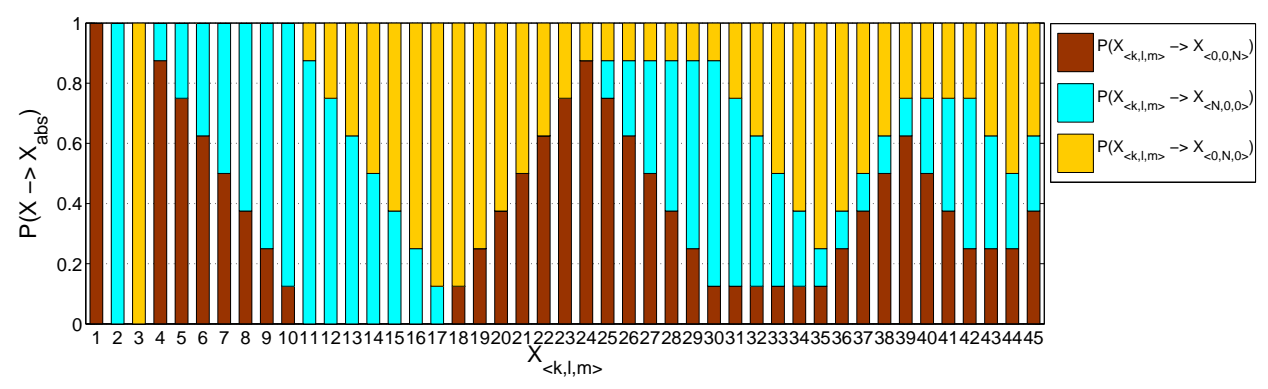

Figure 3.12: The probabilities of reaching the three absorbing states for all initial nodes $X_{\langle k, l, m\rangle}$. Notice that all three final states can be reached only from the inner nodes (numbers 25 to 45 ).

\subsubsection{Bounded Confidence}

Let us now turn to the question of what happens if agents with a certain opinion do not accept to change their opinion after meeting an agent of another given opinion. In the opinion dynamics literature, this is referred to as bounded confidence (Deffuant et al., 2001; Hegselmann and Krause, 2002). From the Markov chain perspective the emergence of opinion polarization becomes a simple consequence of the restrictions posed on the interaction process. As certain transitions are excluded, the state space topology of the Markov chain changes in a way that new absorbing states become present. The respective states correspond to non-consensus configurations, hence, they represent a population with opinion clustering.

As an example, let us assume that agents in opinion state $a$ are not willing to communicate with agents in state $c$ and vice versa, that is to say $\alpha(a, c)=\alpha(c, a)=0$. The corresponding Markov transition matrix $P$ now reads:

$$
P\left(X_{\langle k, l, m\rangle}, X_{\langle k-1, l, m+1\rangle}\right)=P\left(X_{\langle k, l, m\rangle}, X_{\langle k+1, l, m-1\rangle}\right)=0 .
$$

and

$$
P\left(X_{\langle k, l, m\rangle}, X_{\langle k, l, m\rangle}\right)=\left(\frac{k^{2}+l^{2}+m^{2}}{N^{2}}\right)+2\left(\frac{k m}{N^{2}}\right) .
$$

The remaining entries are, as before, given by (3.34) and (3.35). The resulting state space topology is shown in Fig. 3.13, where all horizontal transi- 
tion paths are removed, since those paths correspond to the $a \leftrightarrow c$ opinion changes.

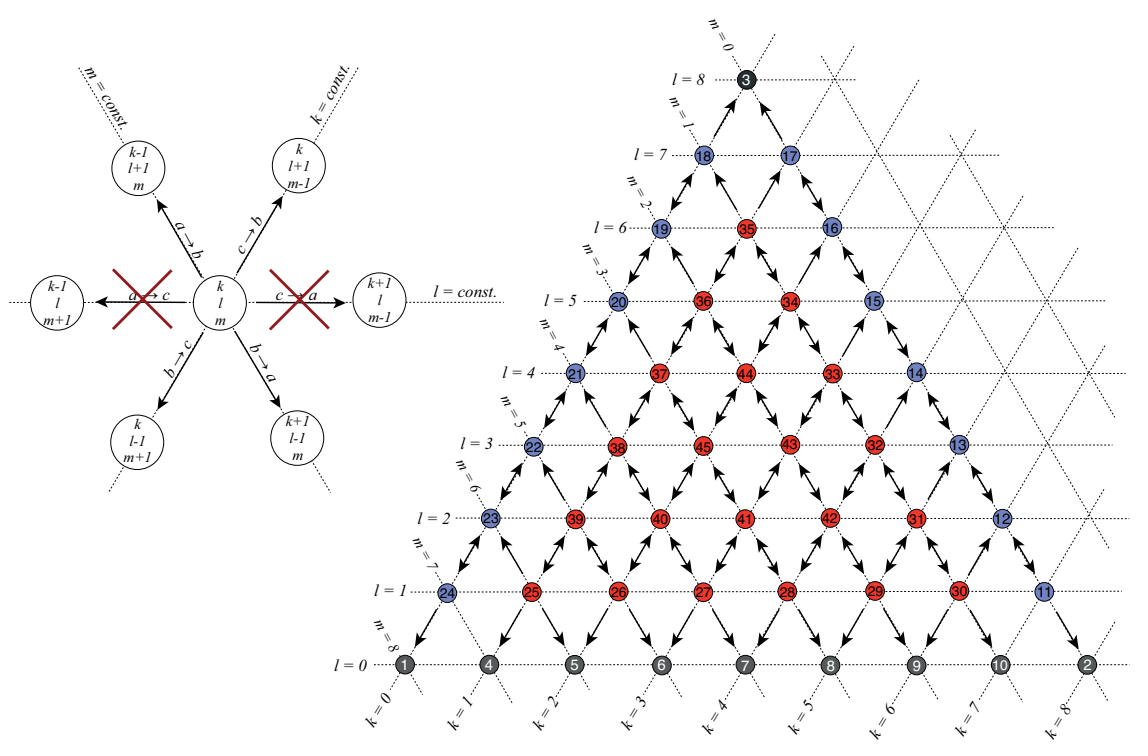

Figure 3.13: Transition structure and state topology of the bounded confidence model for $N=8$. All states on the $l=0$ line (no $b$-agents) are now absorbing states.

For the set of bordering nodes $X_{\langle k, 0, N-k\rangle}: k=1, \ldots, N-1$ with $l=0$ (no $b$-agents) there is no longer any transition that leads away from them, so that all these nodes become absorbing states. The fact that these additional absorbing states $X_{\langle k, 0, N-k\rangle}$ represent opinion configurations with $k$ agents in state $a$ and $N-k$ agents in state $c$ explains why the introduction of interaction restrictions leads to possible final states with opinion polarization. It is noteworthy, however, that the opinion clustering would not be observed if only one of the two transitions, $a \rightarrow c$ or $c \rightarrow a$, were excluded. In this case, there would still be a path leading away from the bordering nodes to one of the nodes $\left(X_{\langle 0,0, N\rangle}\right.$ or $\left.X_{\langle N, 0,0\rangle}\right)$ in the corner of the graph. Such a set-up corresponds to an asymmetric model where the bordering atoms $X_{\langle k, 0, N-k\rangle}: k=1, \ldots, N-1$ become again transient, such that the process eventually leads to the final consensus configurations as previously described. However, in the case that $a \rightarrow c$ but $c \nrightarrow a$ the final configuration $\mathbf{x}=(c c \ldots c)$ would be much more likely than $\mathbf{x}=(a a \ldots a)$, as a consequence of the asymmetry of such a model variant.

As for the unconstrained case, the fundamental matrix can be computed here as well and allows us to calculate the statistical quantities of the model such as absorbing probabilities and times. In Fig. 3.14 the probabilities of a realization starting in one of the transient states ending up in each of the absorbing final states are shown for each initial node (computed again by $B=$ 
$\mathbf{F} R$ ). If the process is in the first 10 nodes at $t=0$, it will remain there forever as all these nodes are absorbing in the bounded confidence case. Notice that nothing changes for the nodes 11 to 24 with respect to the unconstrained case shown in Fig. 3.12. For a system in these configurations the communication constraint has no effect on the dynamics. The six absorbing non-consensus states (numbers 4 to 10 with only $a$ and $c$ opinion supporters) are reachable only from the inner nodes, that is only if all opinions are present initially. It becomes clear that for some of these configurations, the probability of converging to consensus becomes very small (e.g. nodes 25 to 30 ).

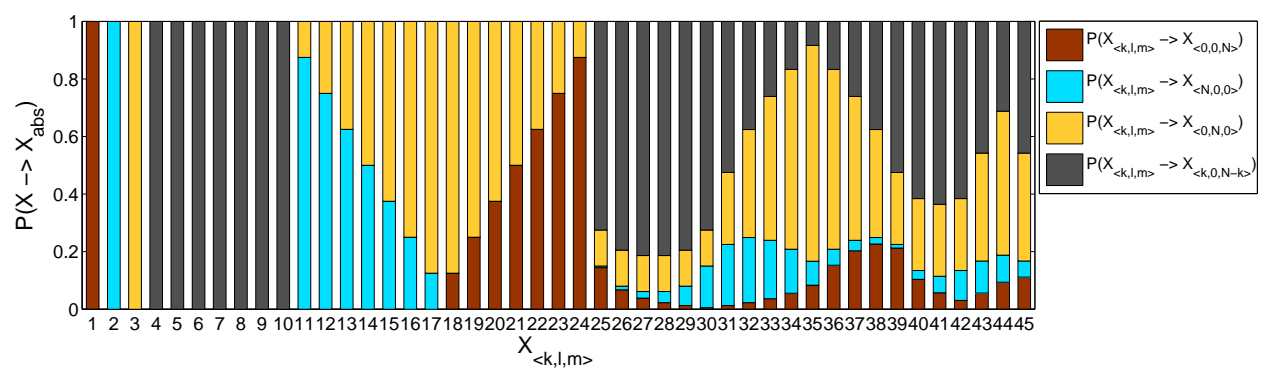

Figure 3.14: The probabilities for all initial nodes $X_{\langle k, l, m\rangle}$ converging to opinion clustering or to the three consensus nodes. Notice again that all final states and the non-consensus states in particular can be reached only from the inner nodes (numbers 25 to 45 ).

Finally, we can compare the mean time before a realization starting in a transient state remains in the transient before absorption for the bounded and the unbounded case. This statistical indicator is represented in Fig. 3.15. Notice that the times for the states 1 to 3 (unbounded) and 1 to 10 (bounded) are zero as in this case the process is absorbed from the very beginning. Again, the non-absorbing two-opinion states (11 to 24) are not affected.

\subsubsection{Non-Lumpability for Further Reduction}

As in the general case of any $\delta$, we can search here for lumpable partitions to further reduce the problem taking the point of view of each »party « associated with opinions $a, b$ or $c$. For the unconstrained case, we have shown in Sec. 3.3.5 that the dynamics from any of these points of view reduces to the $\delta=2$ case. The status of the bounded confidence model is different. From the perspective of opinion $b$ the partition in »supporters « and »opponents « is lumpable, therefore, the system evolves as a binary chain (see next section). This is not the case from the perspectives of opinions $a$ or $c$. For instance, 


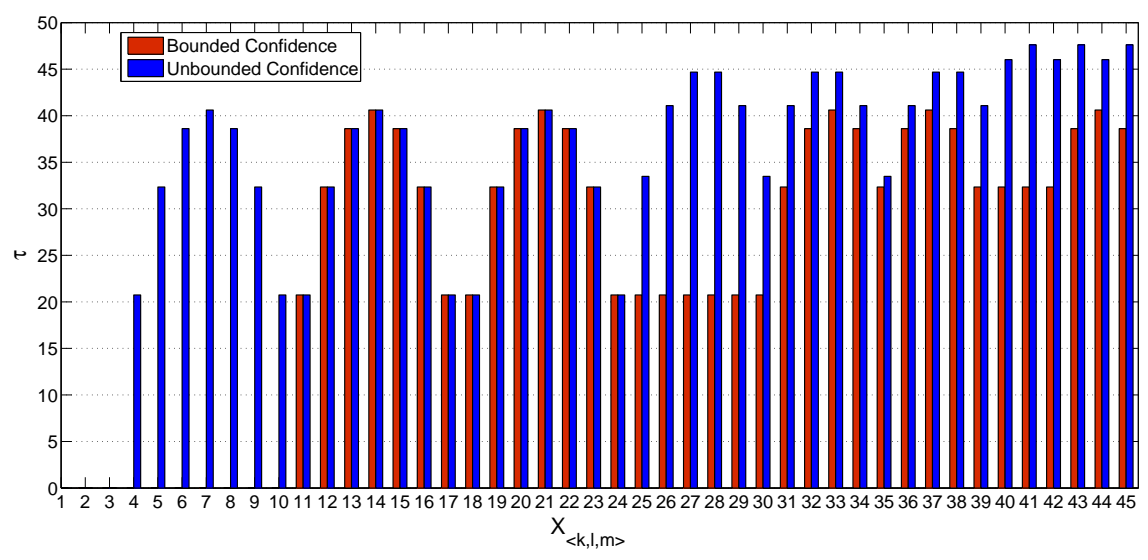

Figure 3.15: Mean times for the transient nodes to reach an absorbing state. Blue bars: unbounded confidence, red bars: bounded confidence with $a \nLeftarrow s$. Labels of the nodes are explained in the text.

from the point of view of opinion $a$, the corresponding partition reads:

$$
Y_{r}^{a}=\bigcup_{l+m=N-r} X_{\langle r, l, m\rangle}, \quad r=0,1, \ldots, N .
$$

and

$$
P\left(X_{\langle r, l, m\rangle}, Y_{r+1}^{a}\right)=\frac{r l}{N^{2}} .
$$

It turns out that the chain formed by the $Y_{r}^{a}, r=0,1, \ldots, N$ is not a Markov chain since the r.h.s. of (3.42) depends on $l$ and not only on $r$ (Kemeny and Snell, 1976).

We see that the introduction of bounded confidence in this model leads to memory effects due to the fact that an agent switching from opinion $a$ to opinion $c$ necessarily goes through a visit to opinion $b$ for at least one time step. Therefore, the probability of this transfer will depend on the number of supporters of opinion $b$ at that time.

\subsubsection{Transient Behavior with Bounded Confidence}

As noted above, a further reduction of the Markov chain is possible if the dynamics are considered from the perspective of $»$ party $« b$. The corresponding partition reads

$$
Y_{r}^{b}=\bigcup_{k+m=N-r} X_{\langle k, r, m\rangle}, \quad r=0,1, \ldots, N
$$

and the transition probabilities are

$$
P\left(X_{\langle k, r, m\rangle}, Y_{r+1}^{b}\right)=\frac{r(N-r)}{N^{2}} .
$$


As discussed in Sec. 3.3.5, the probability to converge to $Y_{N}^{b}=\{(b b \ldots b)\}$ is $1 / \delta=1 / 3$ and the probability to end up in one of the configurations in $Y_{0}^{b}$ is $2 / 3$, provided that the model is initialized with an equal number of $a$, $b$ and $c$ agents. Notice that contrary to the unbounded confidence case the process is really in its final state whenever $r=0$ as all configurations in $Y_{0}^{b}$ are absorbing.

The convergence times (see Fig. 3.15 for a small system) are composed of the (relatively short) time needed to end up in the class of states $Y_{0}^{b}$ including those of opinion polarization and the (relatively long) time needed to converge to $Y_{N}^{b}$. In the following, we use a transformation proposed in Kemeny and Snell (1976), 64/65, in order to assess the two times independently. Notice that all the results obtained in this section are also applicable to the binary VM (with $X_{k} \equiv Y_{k}^{b}$ ) to study the effects of initial opinion bias.

The basic idea is to $\gg$ compute all probabilities relative to the hypothesis that the process ends up in the given absorbing state« (ibid, 64). This leads to a new absorbing chain with the specified state as the single absorbing state. In fact, for our purpose, it is not necessary to completely determine the transition matrix for that new chain as the fundamental matrix of the original process $(\mathbf{F})$ can be used directly to compute the fundamental matrix of the new chain $(\tilde{\mathbf{F}})$. Let $B=\mathbf{F} R$ where $\mathbf{F}$ is the fundamental matrix of the binary chain (3.22) and $R$ the respective $2 \times N$ submatrix of the canonical form (3.19). The elements $b_{1 j}\left(b_{2 j}\right)$ of $B$ correspond to the exit probabilities of the process started in $j$ to end up in $X_{0} \equiv Y_{0}^{b}\left(X_{N} \equiv Y_{N}^{b}\right)$. Recall that $b_{1 j}=(N-j) / N$ and $b_{2 j}=j / N$ (see Sec. 3.3.3). Now let $D_{0}$ be a diagonal matrix with $d_{j j}=b_{1 j}$ and respectively define $D_{N}$ as $d_{j j}=b_{2 j}$. Then, according to Kemeny and Snell (1976), 65, the fundamental matrices of the new chains with absorbing state $X_{0} \equiv Y_{0}^{b}$ and $X_{N} \equiv Y_{N}^{b}$ respectively is given by

$$
\begin{array}{r}
\tilde{\mathbf{F}}_{0}=D_{0}^{-1} \mathbf{F} D_{0} \\
\tilde{\mathbf{F}}_{N}=D_{N}^{-1} \mathbf{F} D_{N} .
\end{array}
$$

In our case with the fundamental matrix given in Eq. 3.22 we obtain

$$
\left(\tilde{\mathbf{F}}_{0}\right)_{i j}=b_{1 j} \frac{F_{i j}}{b_{1 i}}=\left\{\begin{array}{cl}
\frac{i N(N-j)}{j(N-i)} & : i \leq j \\
N & : i>j
\end{array}\right.
$$

and

$$
\left(\tilde{\mathbf{F}}_{N}\right)_{i j}=b_{2 j} \frac{F_{i j}}{b_{2 i}}=\left\{\begin{array}{cc}
N & : i \leq j \\
\frac{j N(N-i)}{i(N-j)} & : i>j
\end{array} .\right.
$$

The fundamental matrices $\tilde{\mathbf{F}}_{0}$ and $\tilde{\mathbf{F}}_{N}$ allow for a very good understanding of the average behavior of the model. $\tilde{\mathbf{F}}_{0}$ encodes the mean number of 
steps that the realizations which eventually converge to $Y_{0}^{b}$ pass through any state $Y_{r}^{b}$, and $\tilde{\mathbf{F}}_{N}$ informs us about the mean behavior of realizations that end up in $Y_{N}^{b}$. For instance, we can compute the mean convergence time to each absorbing state independently. For convergence to $Y_{0}^{b}$ from the initial state $Y_{r}^{b}$ we have

$$
\tilde{\tau}_{0}(r)=r N+\sum_{j=r+1}^{N} \frac{r N(N-j)}{j(N-r)}
$$

and for convergence to uniformity corresponding to $Y_{N}^{b}$

$$
\tilde{\tau}_{N}(r)=(N-r) N+\sum_{j=1}^{r-1} \frac{j N(N-r)}{r(N-j)}
$$

For a system of 100 agents these times are shown in Fig. 3.16. It becomes clear that the mean convergence times to $Y_{0}^{b}$ and $Y_{N}^{b}$ are equal if the initial situation is unbiased, that is, if there are $r=N / 2$ agents with attribute $b$ and $N / 2$ agents in the other two states $a$ or $c$. However, with an increasing initial bias, there is an increasing gap between average convergence time to one or the other absorbing state. For the system with three possible attributes $a, b$ and $c$ and random initial conditions the initial number of $b$ agents is around $N / 3 \approx 33$. This is illustrated by the dashed vertical line. In that case, the mean convergence time for realizations that end up in possible polarized configurations with only $a$ and $c$ agents becomes considerably smaller compared to the configuration with all agents in state $b$.

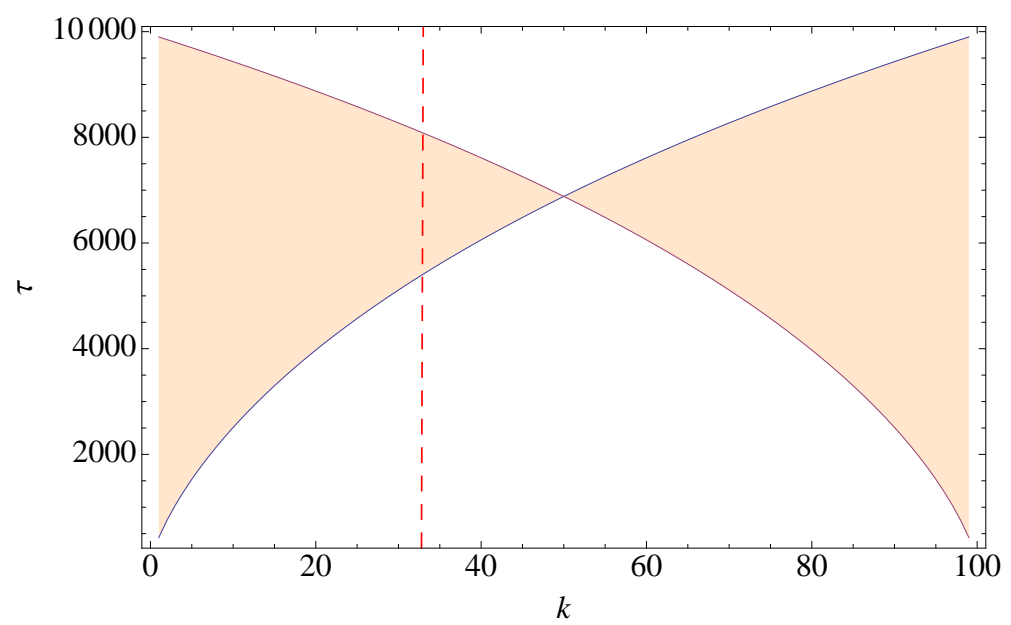

Figure 3.16: Mean convergence times to $X_{0} \equiv Y_{0}^{b}\left(\right.$ red) and $X_{N} \equiv Y_{N}^{b}$ (blue) independently. The vertical dashed line represents the initial bias for the model with $\delta=3$.

$\tilde{\mathbf{F}}_{0}$ and $\tilde{\mathbf{F}}_{N}$ enable moreover to study the transient behavior of the model with initial bias in more detail. For a system of 100 agents, Fig. 3.17 shows 

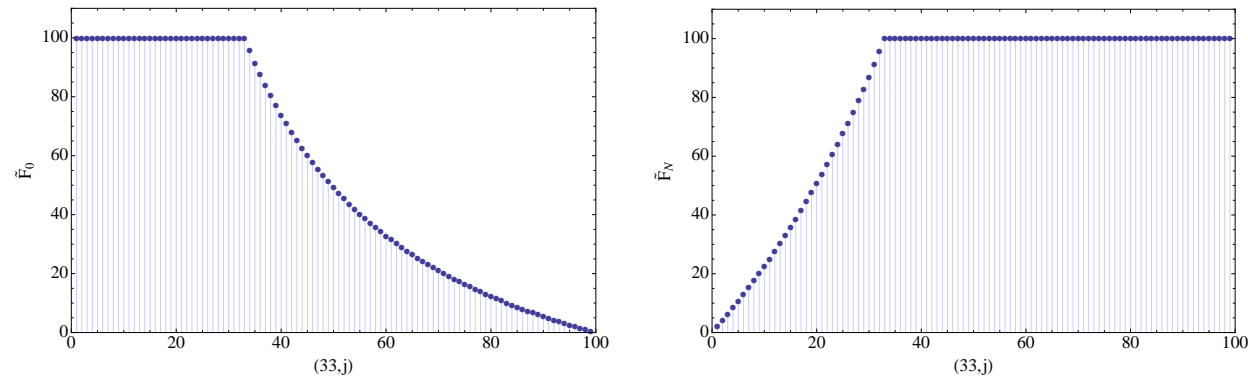

Figure 3.17: Mean number of steps a process that eventually converges to $Y_{0}^{b}$ (l.h.s.) and $Y_{N}^{b}$ (r.h.s.) is in the transient states for a system of 100 agents and an initial number of $r=33$ agent in state $b$.

the mean number of steps that a process ending up in $Y_{0}^{b}$ (l.h.s.) and $Y_{N}^{b}$ (r.h.s.) is in the transient states provided the model is initialized with 33 agents in $b$ and 67 agents in $a$ or $c$. This information is encoded in the 33rd row of $\tilde{\mathbf{F}}_{0}$ and $\tilde{\mathbf{F}}_{N}$ respectively. We first comment on the l.h.s. showing the mean behavior of realizations ending up in an absorbing configuration where only $a$ and $c$ agents remain $\left(Y_{0}^{b}\right)$. The figure shows that, in average, all the transient states that are closer to $Y_{0}^{b}$ than the initial configuration in $Y_{33}^{b}$ are met $N$ times. Naturally, the states $»$ to the right « are encountered less frequently. It should be clear that the entries of $\tilde{\mathbf{F}}_{0}$ should not be read as the mean behavior of every single realization, but rather as the average behavior over a large series of realizations. For instance, the mean number of steps to $Y_{99}^{b}$, which is very close to the opposite absorbing state, is approximately $1 / 2$. However, this does not mean that every second realization approaches the opposite absorbing state so closely. It rather means that there are rare realizations that take that way, and once they are at the opposite extreme, these realizations have a high chance to stay there for some while. In fact, the fundamental matrix $\tilde{\mathbf{F}}_{0}$ tells us that, once a realization reached $Y_{99}^{b}$, the mean number of returns to that state is $N-1$. The interpretation of the r.h.s. $\left(\tilde{\mathbf{F}}_{N}\right)$ goes in the same way.

Finally, the probability distribution of convergence times to one or the other absorbing state can be computed easily for a given $N$. In this computation, we first compute the respective matrices $\tilde{Q}_{0}$ as $\left(\tilde{Q}_{0}\right)_{i j}=\left(b_{1 j} / b_{1 i}\right) Q_{i j}$ and $\tilde{Q}_{N}$ as $\left(\tilde{Q}_{N}\right)_{i j}=\left(b_{2 j} / b_{2 i}\right) Q_{i j}$. This is in complete analogy to the computation of the independent fundamental matrices and follows the work of (Kemeny and Snell, 1976, 64-65). The computation of the probability distribution is then based on the evaluation of powers of $\tilde{Q}$ as done in Sec. 3.3.2. The result is shown in Fig. 3.18. For comparison, the distribution of convergence times to either absorbing state (dashed, red) is shown for $r=33$. All in all, this shows how the general convergence behavior is a composite of the two different convergence trends obtained by considering the two absorbing states independently. 

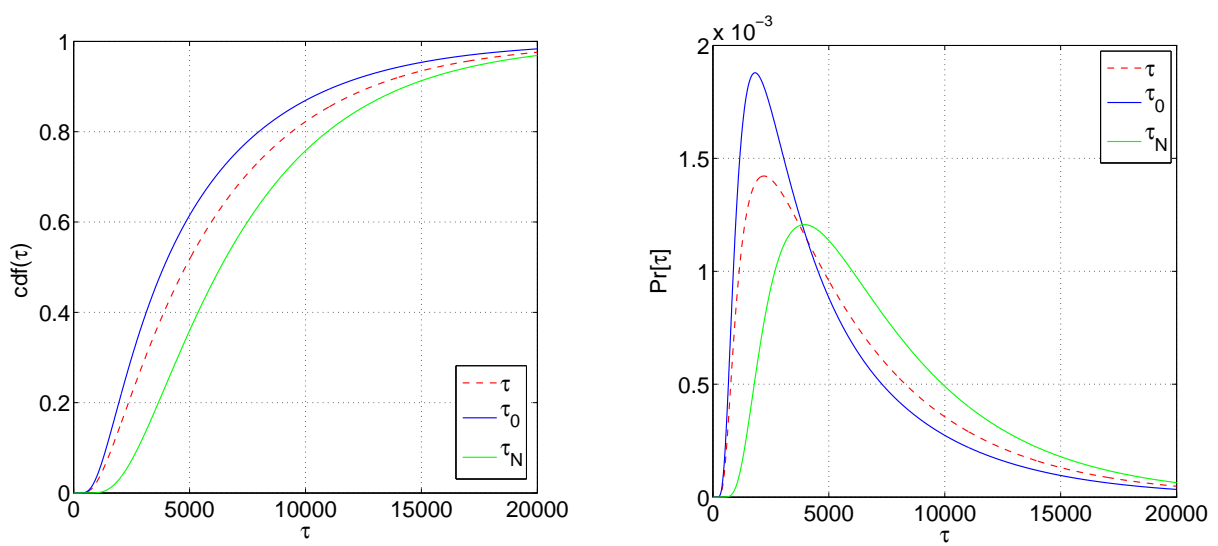

Figure 3.18: Probability distribution of convergence times for a system of 100 agents when started with $r=33$. Convergence to $Y_{0}^{b}, \tau_{0}$, is considerably faster than convergence to $Y_{N}^{b}, \tau_{N}$.

\subsection{Simple Generalizations}

We first mention an easy generalization of the existence of absorbing states for the case of bounded confidence in a model with any number $\delta$ of different opinions. In order to get non-consensual absorbing states it is necessary and sufficient that a subset of opinions is mutually incommunicable. In this case all the states belonging to the simplex generated by the mutually incommunicable opinions become absorbing. It is worthwhile noticing that absorbing states may appear in different clusters of simplexes provided that the corresponding opinions are related by chains of communicating links. An example of this type appears for $\delta=3$ if $(a \leftrightarrow b)$ and $(a \leftrightarrow c)$ but $(b \leftrightarrow c)$ where the absorbing states are either the simplex with only $a$ and $b$ or with only $a$ and $c$ opinions.

Another interesting issue concerns agent models with vectorial (or equivalently matrix or table) individual attribute space. Suppose that at each time step each agent $i$ is characterized by a list of $q$ attributes, where the first attribute may take $n_{1}$ possible values, the second attribute $n_{2}$ values and so on up to the $q^{\text {th }}$ attribute with $n_{q}$ possible values. The corresponding ABM can then be easily built as in Sec. 3.3.4 by taking $\delta=n_{1} \times n_{2} \times \cdots \times n_{q}$. As long as one is interested in following the macrodynamics obtained by lumping all agent configurations with an equal attribute frequency for all the $\delta$ attributes, the reduction proposed in Sec. 3.3.5 also applies. Moreover, absorbing non-consensual states will appear in exactly the same way as described above as a consequence of bounded confidence.

For this vectorial opinion model there is, however, an unexpected subtlety when we are interested in the macrodynamics of the agents ranked by only one of their attributes, for instance, if the agents are separated in $n_{1}$ dif- 
ferent groups according to the number of agents sharing their first attribute. Then, the partition is no longer lumpable, and therefore the evolution of the corresponding random variables (for instance, the number of elements of each group) is not a Markov chain. Again, in this case, new memory effects may appear from this choice of aggregation to build the macrodynamics. The proof can be done as in (3.41) and (3.42).

\subsection{Discussion}

In this chapter, we considered a class of ABMs from a Markovian perspective and derive explicit statements about the possibility of linking a microscopic agent model to the dynamical processes of macroscopic observables that are useful for a precise understanding of the model dynamics. We showed that the class of single-step ABMs may be described as random walks on regular graphs and that the symmetries of the corresponding transition structure is related to lumpable macro partitions. In this way the dynamics of collective variables may be studied, and a description of macro dynamics as emergent properties of micro dynamics, in particularly during transient times, is possible.

Using Markov chain computations, we obtain a very detailed understanding of the VM with homogeneous mixing. On the one hand, the computation of the fundamental matrix of the macro chain provides us with precise knowledge about the mean transient behavior, on the other, it also tells us that some care must be taken in order to relate those mean quantities to single realizations of the model. Regarding convergence times, full information (probability distribution of convergence times) is provided by numerical integration over the transient states which gives a better idea of the transient behaviors that single realizations may exhibit. The analysis is extended to the general (multi-state) VM, the analysis of which is reducible to the binary case in the absence of interaction constraints. On the other hand, similarity constraints as bounded confidence or assortative mating lead to additional absorbing states in the macro chain. This shows that opinion polarization is a direct consequence of bounded confidence (see Banisch and Araújo, 2012 for a biological interpretation in terms of sympatric speciation).

In our context, the random map representation (RMR) of a Markov process helps to understand the role devoted to the collection of (deterministic) dynamical rules used in the model from one side and of the probability distribution $\omega$ governing the sequential choice of the dynamical rule used to update the system at each time step from the other side. The importance of this probability distribution, often neglected, is to encode the design of the social structure of the exchange actions at the time of the analysis. Not only, then, are features of this probability distribution concerned with the social context the model aims to describe, but they are also crucial in pre- 
dicting the properties of the macro dynamics. If we decide to remain at a Markovian level, then the partition, or equivalently the collective variables, to be used to build the model should be compatible with the symmetry of the probability distribution $\omega$.

This is what makes homogeneous mixing (and respectively, the complete graph) so special because the full permutation invariance is realized $\left(\operatorname{Aut}\left(K_{N}\right)=\mathcal{S}_{N}\right)$. On the other hand, an important mark of ABMs is their ability to include arbitrary levels of heterogeneity and stochasticity (or uncertainty) into the description of a system of interacting agents. In a sense, the partition of the configuration space defining the macro level of the description has to be refined in order to account for an increased level of heterogeneity or a falloff in the symmetry of the probability distribution. It is, however, clear that, in absence of any symmetry, there is no other choice for this partition than to stay at the micro level and, in this sense, no Markovian description of a macro level is possible in this case. This will be spelled out in detail in the next chapter. 


\section{From NeTWORK Symmetries TO MARKOV PROJECTIONS}

In the previous chapter, we have seen that an ABM defines a process of change at the individual level - a micro process - by which in each time step one configuration of individuals is transformed into another configuration. For a class of models we have shown this micro process to be a Markov chain on the space of all possible agent configurations. Moreover, we have shown that the full aggregation - that is, the re-formulation of the model by mere aggregation over the individual attributes of all agents - may give rise to a new process that is again a Markov chain, however, only under the rather restrictive assumption of homogeneous mixing. Heterogeneities in the micro description, in general, destroy the Markov property of the macro process obtained by such a full aggregation.

The question addressed in this chapter is how to derive Markovian coarsegrainings (Markov projections) if the assumption of homogeneous mixing is relaxed. In other words, how must the micro model and the projection construction be so that the projected system is still a Markov chain? We develop a tool which relates symmetries in the interaction topology to partitions of the configuration space with respect to which the micro process is lumpable. In effect, this leads to a refinement of the full aggregation which exploits all the dynamical redundancies that have its source in the agent network on which the model is implemented. Notably, the result is stated in terms of the symmetries of the agent network which is much simpler than the micro chain on the configuration space where the aggregation process (lump) is achieved.

The theoretical ideas presented here have been made available under Banisch and Lima (2012). A more detailed version containing the results for the example studied in Sec. 4.4 is under review (Banisch and Lima, 2013). The results have been presented at a series of conferences (Banisch et al., 2013; Banisch, 2013, a,b). 


\subsection{Interaction Heterogeneity and Projection Re- finement}

Let us begin this chapter with the simple example that is running through this thesis. Consider the VM with three agents on different networks defined by a $3 \times 3$ adjacency matrix $A$ with $a_{i j}=1$ whenever $i$ and $j$ are connected. As before, in the iteration process, an agent pair $(i, j)$ is chosen at random out of the set of all agent pairs with $a_{i j}=1$ and the first adopts the state of the second. Notice that an alternative way of realizing the agent update is to first choose an agent $i$ and then choose another agent $j$ out of all agents connected to $i$. The former is called link update and the latter node update dynamics and we shall see that this can lead to different probability distributions $\omega$. We mainly consider link update in this chapter, but comment on the differences between the two variants in Sec. 4.2.

We first consider the complete graph defined by $a_{i j}=1$ whenever $i \neq j$ and $a_{i i}=0$. Notice that in that case, the two update variants are lead to the same $\omega(i, j)$. Namely, the probability of a pair $(i, j)$ to be chosen is for every pair $\omega=1 / 6$. That is, except for the exclusion of self-choice (with $\omega(i, i)=0)$ it leads to the case dealt with in the previous chapter. Fig. 4.1 briefly recalls the respective Markov chain formulation and projection by illustrating (i.) the connectivity structure $\omega(i, j)=\omega ; \forall i \neq j$, (ii.) the micro chain this leads to along with the transition rates, and (iii.) the resulting macro chain.

In order to go beyond complete homogeneity let us consider what happens to that picture of one link is removed. Therefore, let us assume that $a_{23}=a_{32}=0$. Under link update this leads to the following interaction probabilities: $\omega(1,2)=\omega(2,1)=\omega(1,3)=\omega(3,1)=\omega=1 / 4$, and $\omega(2,3)=\omega(3,2)=0$. This topology, the resulting micro chain and the probabilistic effects on the macro level are shown in Fig. 4.2.

It becomes clear that the introduction of interaction heterogeneity translates into irregularities in the probabilistic structure of the micro chain in a way that the symmetry condition in Theorem 3.2.2, $\hat{P}(\mathbf{x}, \mathbf{y})=\hat{P}(\hat{\sigma}(\mathbf{x}), \hat{\sigma}(\mathbf{y}))$, is violated for the macro partition $\mathbf{X}=\left(X_{0}, X_{1}, X_{2}, X_{3}\right)$. In other words, it leads to the non-lumpability of the partition $\mathbf{X}=\left(X_{0}, X_{1}, X_{2}, X_{3}\right)$. As shown in Fig. 4.2 the transition probabilities at the macro level are not uniquely defined and depend upon the respective micro configuration. Consider, as an example, the transitions from $X_{2}$ to $X_{3}$. The probability (3.7) of a transition form configuration $(\square \square \square)$ to $(\square \square \square)$ is $\hat{P}(\square \square \square, \square \square \square)=$ $\omega(1,2)+\omega(1,3)=2 \omega$, whereas $\hat{P}(\square \square, \square \square \square)=\omega(2,1)+\omega(2,3)=\omega$ and $\hat{P}(\square \square \square, \square \square \square)=\omega(3,1)+\omega(3,2)=\omega$. While all these probabilities are equal for the complete graph (as $\omega(i, j)=\omega: \forall i, j$ ) they are not all equal if one or two connections are absent which violates the lumpability condition.

Deriving a partition such that the micro process projected onto it is a 


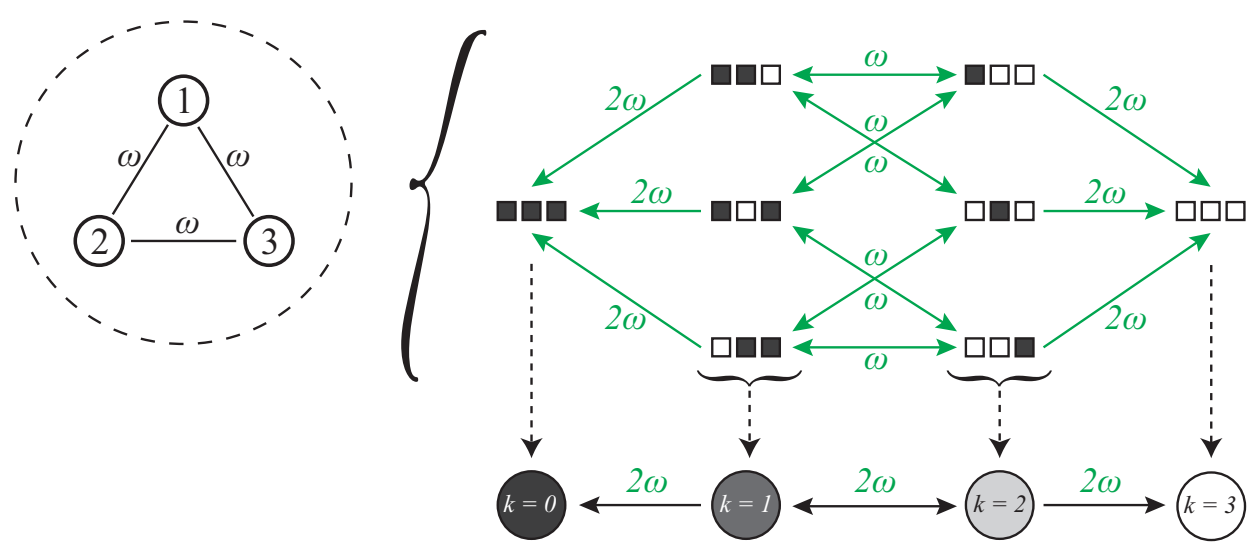

Figure 4.1: Probabilistic structure of the model with three agents on the complete graph.

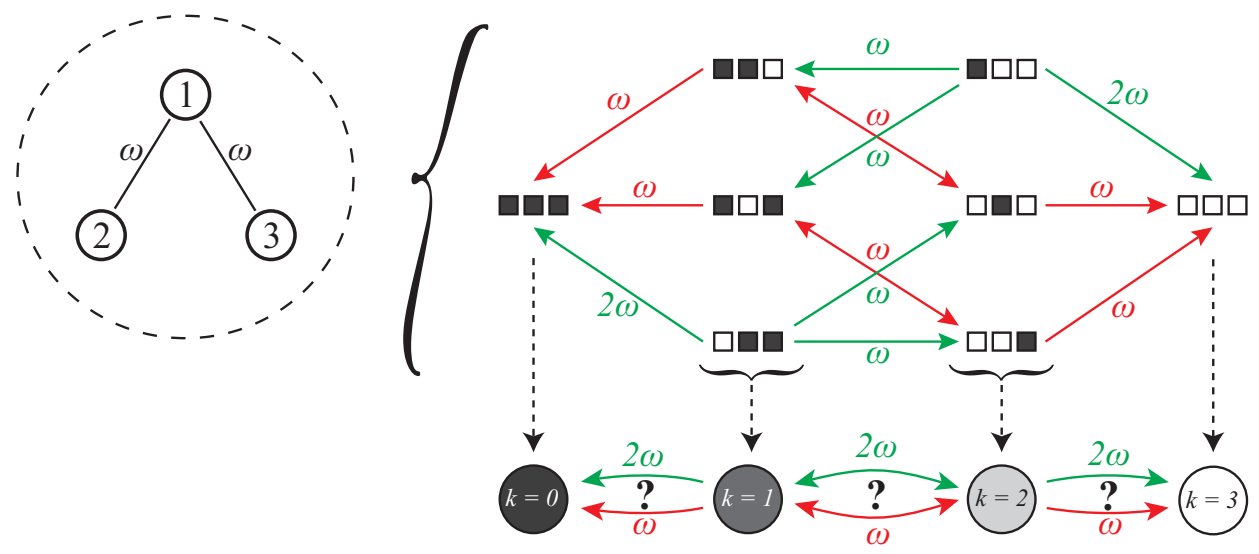

Figure 4.2: Probabilistic structure of the model with three agents if the connection between 2 and 3 is absent.

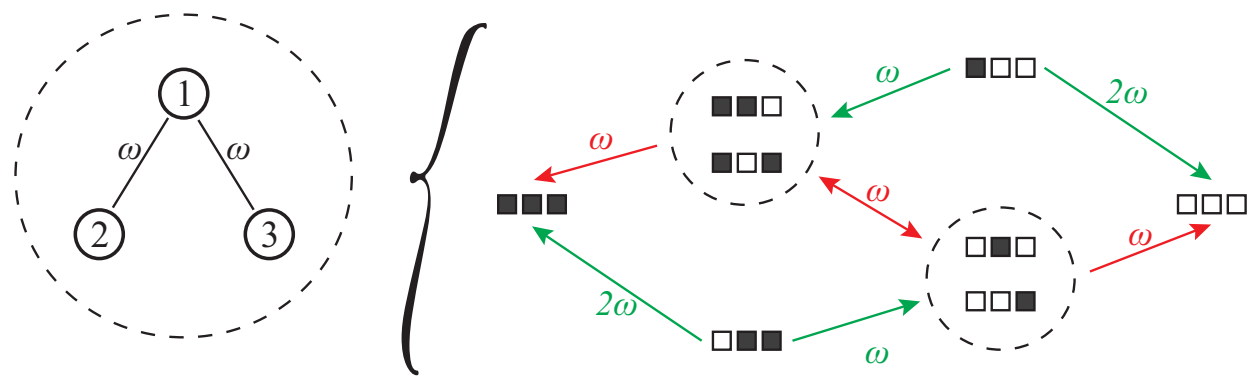

Figure 4.3: Refinement of the partition that preserves Markovianity. 
Markov chain requires a refinement of the aggregation procedure. For the example considered here the respective refined partition is shown in Fig. 4.3.

The main purpose of this chapter is to develop a systematic approach to this projection refinement by exploiting all the dynamical redundancies resulting from the symmetries of agent network. Network symmetries can be used to identify bundles of micro configurations that can be interchanged without changing the hypercubic micro chain. Our example may provide a first intuition. The interaction graph in our example has a symmetry such that the agents 2 and 3 can be permuted without affecting the connectivity structure, (i.e., $\left.A u t_{\omega}=(1)(23)\right)$. This symmetry imposes a symmetries in the hypercube graph associated to the micro process such that the configurations $(\square \square \square$ ) and ( $\square \square \square$ ) with $k=2$ and respectively ( $\square \square$ ) and $(\square \square)$ with $k=1$ can be permuted without affecting the transition structure. See also Fig. 4.4. In this simple example, therefore, the previous macro atoms $X_{2}$ (and $X_{1}$ ) must be refined such that the sets of configurations $\{(\boldsymbol{\square} \square),(\boldsymbol{\square} \square)\}$ (respectively $\{(\square \square),(\square \square \square)\}$ ) on the one hand and $\{(\square \square)\}$ (respectively $\{(\square \square)\}$ ) on the other form different sets in a Markovian partition.

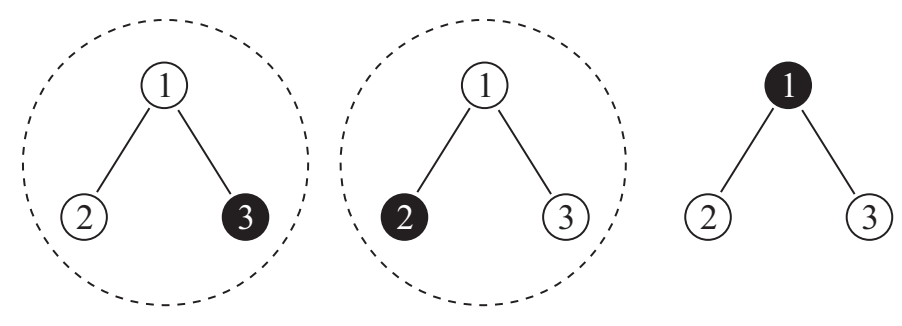

Figure 4.4: The 3 different configurations ( $\square \square \square$ ), ( $\square \square \square$ ) and ( $\square \square \square$ ) of length 3 with one agent in $\boldsymbol{\square}$ and two in $\square(k=2)$. The first two configurations ( $\square \square \square$ ) and $(\square \square \square)$ are what we will call macroscopically equivalent.

\subsection{Social Structure at the Micro Level}

The effect of different social networks on the dynamics of ABMs plays an increasingly important role in the research of these models. Certain aspects of the model behavior may sometimes be very different when implemented on different topologies. One might be surprised that in the consideration of the micro level dynamics (Sec. 3.1, previous chapter) nothing is said about how different agent networks incorporate into this framework. The simple reason is that the role of networks in the models is essentially to determine the interaction probabilities of agents and that we consider that kind of information via the probability distribution $\omega$.

For instance, in the VM two agents $(i, j)$ linked in the network are chosen at random. From the network it is possible to infer directly the respective 
probability $\omega(i, j)$. As mentioned earlier, there are two different ways of agent choice: first, one can first choose an agent $i$ and then choose another agent $j$ out of its neighborhood (node update dynamics); second, both agents are chosen at one instance by the choice of an edge in the network (link update dynamics). In general, the two modes of agent choice lead to a different $\omega(i, j)$. Node update leads to

$$
\omega(i, j)=\frac{1}{N} \frac{1}{k_{i}}
$$

where $k_{i}$ is the degree of agent $i$. For the second version with link choice, on a graph with adjacency matrix $A$ the probability $\omega(i, j)$ is

$$
\omega(i, j)=\frac{a_{i j}}{|\mathbf{E}|},
$$

where $a_{i j}$ is the element in $A$ corresponding to the edge $(i, j)$ and $|\mathbf{E}|$ is the total number of edges in the graph.
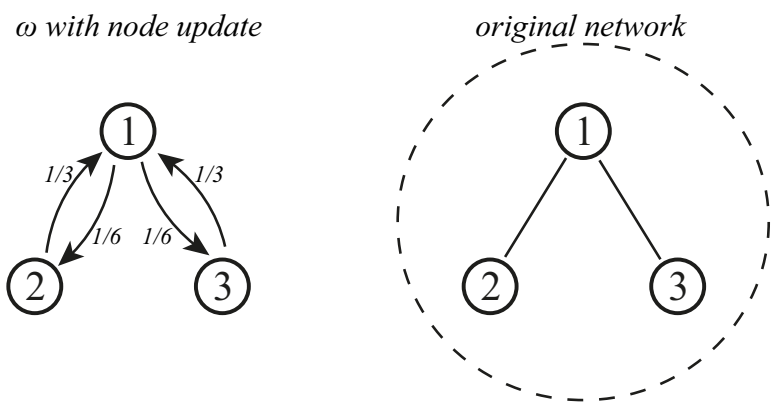

$\omega$ with link update

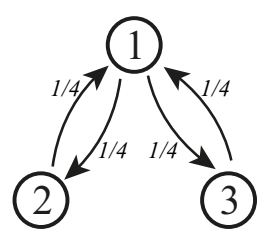

Figure 4.5: Node versus link update in the example considered above.

Fig. 4.5 illustrates the differences in the probability distribution $\omega$ for node and link update using the small example considered in the previous section. Notice that in any case $\sum \omega(i, j)=1$ for it is a distribution over agent choices. Notice moreover, that the symmetry (1)(23) is preserves for the two update schemes.

The notion of $\omega$ is quite general and allows also to incorporate other types of social structure. There may be cases in which agents are heterogeneous with respect to certain static characteristics: for instance, if they belong to different ethnical groups or working classes. This might effect not only there likeliness to meet in the model, but also the choice probabilities for different behavioral options, that is, the behavior of agents within their group may be different from the agent behavior across different groups. All those effect are encoded into the probability distribution $\omega$ along with the social network of agents. 


\subsection{Markovian Aggregation}

For those reasons, it is convenient to formulate the theoretical ideas presented in this section in terms of the probability distribution $\omega$. We first define the notion of macroscopic equivalence, then we consider the VM and finally we discuss the generalization to the class of models with single-step dynamics (see Sec. 3.1.3).

\subsubsection{Macroscopic Equivalence}

Let $(\boldsymbol{\Sigma}, \hat{P})$ be a micro chain corresponding to an ABM. Let $\mathcal{M}$ be a partition of the configuration space $\boldsymbol{\Sigma}$ with respect to which the micro process is lumpable. For further convenience we define the following notion of macroscopic equivalence:

Definition 4.3.1 Two configurations $\mathrm{x}$ and $\mathrm{x}^{\prime}$ are macroscopically equivalent if the lumpability condition (Kemeny and Snell, 1976, Thm. 6.3.2)

$$
\hat{p}_{\mathbf{x} Y}=\sum_{\mathbf{y} \in Y} \hat{P}(\mathbf{x}, \mathbf{y})=\sum_{\mathbf{y} \in Y} \hat{P}\left(\mathbf{x}^{\prime}, \mathbf{y}\right)=\hat{p}_{\mathbf{x}^{\prime} Y}
$$

is satisfied for all $Y \in \mathcal{M}$. Then $\mathbf{x}$ and $\mathbf{x}^{\prime}$ belong to the same atom $X$ of the partition $\mathcal{M}$.

The notion of macroscopic equivalence is motivated by the fact that two macroscopically equivalent configurations contribute in exactly the same way to the dynamical behavior of the macro process on $\mathcal{M}$. It is important to notice that macroscopic equivalence is inherently linked to a partition $\mathcal{M}$, that is, with a macro description of the process, because two configurations that are equivalent with respect to one partition might not be with respect to another.

\subsubsection{The Voter Model}

Let $A_{u} t_{\omega}(N)$ be the subgroup of the permutations $\sigma$ acting on the set $\mathbf{N}$ of agents such that $\omega(\sigma i, \sigma j)=\omega(i, j)$ for all $i, j \in \mathbf{N}$. To each $\sigma \in \operatorname{Aut}_{\omega}(N)$ we associate a $\hat{\sigma}$ which is a bijection on the configuration space $\boldsymbol{\Sigma}$. If $\mathbf{x} \in \boldsymbol{\Sigma}$ with $\mathbf{x}=\left(x_{1}, \ldots, x_{i}, \ldots, x_{N}\right)$ then

$$
\hat{\sigma}(\mathbf{x})=\left(x_{\sigma 1}, \ldots, x_{\sigma i}, \ldots, x_{\sigma N}\right) .
$$

We now define a partition $\mathcal{M}_{\omega}$ of $\boldsymbol{\Sigma}$ using $A u t_{\omega}(N)$. Two configurations $\mathbf{x}, \mathbf{x}^{\prime} \in \boldsymbol{\Sigma}$ belong to the same atom of the partition $\mathcal{M}_{\omega}$ iff there is a $\sigma \in$ $\operatorname{Aut}_{\omega}(N)$ such that $\mathbf{x}^{\prime}=\hat{\sigma}(\mathbf{x})$. Clearly this is an equivalence relation and therefore it defines a partition on $\boldsymbol{\Sigma}$. 
Proposition 4.3.1 The partition $\mathcal{M}_{\omega}$ is lumpable for the agent model on $\boldsymbol{\Sigma}$ with agent choice based on $\omega$ and therefore the corresponding projected process is a Markov chain.

Proof. Consider the distribution of interaction probabilities $\omega$ and its permutation group of symmetries $\operatorname{Aut}_{\omega}(N)=\{\sigma: \omega(\sigma i, \sigma j)=\omega(i, j), \forall i, j \in$ $\mathbf{N}$ \}. Suppose we know (at least) one configuration (the generator) $\mathbf{x}^{k} \in \boldsymbol{\Sigma}$ for each $X_{k} \subset \boldsymbol{\Sigma}$ and construct the partition $\mathcal{M}_{\omega}=\left(X_{1}, \ldots, X_{k}, \ldots\right)$ by

$$
X_{k}=\operatorname{Aut}_{\omega}(N) \circ \mathbf{x}^{k}=\bigcup_{\forall \hat{\sigma}} \hat{\sigma}\left(\mathbf{x}^{k}\right) .
$$

A necessary and sufficient condition for lumpability is that the transition probability from a configuration $\mathrm{x} \in X_{k}$ to any atom $X_{s} \in \mathcal{M}_{\omega}$ be the same for all $\mathrm{x} \in X_{k}$ (Kemeny and Snell, 1976, Thm. 6.3.2). That is, we have to show macroscopic equivalence, Eq. (4.3.1), for the pairs of configurations $\mathbf{x}$ and $\hat{\sigma}(\mathbf{x})$. By Theorem 3.2.2 we know that this is satisfied whenever $\hat{P}(\mathbf{x}, \mathbf{y})=\hat{P}(\hat{\sigma}(\mathbf{x}), \hat{\sigma}(\mathbf{y}))$ for any $\sigma \in A u t_{\omega}(N)$.

In the $\mathrm{VM}$, for the case that $x \stackrel{i}{\sim} y$ we know that $x_{j}=y_{j}$ for all $j$ except $i$ and that the transition requires the choice of an edge $(i,$.$) . Denoting x_{i}=s$ and $y_{i}=\bar{s}$ we rewrite Eq. (3.7) as

$$
\hat{P}(\mathbf{x}, \mathbf{y})=\sum_{j:\left(x_{j}=\bar{s}\right)} \omega(i, j)
$$

If $\mathbf{x} \stackrel{i}{\sim} \mathbf{y}$ it is easy to show that $\hat{\sigma}(\mathbf{x}) \stackrel{\sigma i}{\sim} \hat{\sigma}(\mathbf{y})$ and we know that $s=\hat{\sigma}\left(x_{\sigma i}\right) \neq$ $\hat{\sigma}\left(y_{\sigma i}\right)=\bar{s}$. The transition therefore requires the choice of an edge $(\sigma i,$.$) .$ We obtain

$$
\hat{P}(\hat{\sigma}(\mathbf{x}), \hat{\sigma}(\mathbf{y}))=\sum_{k:\left(\sigma\left(x_{k}\right)=\bar{s}\right)} \omega(\sigma i, k) .
$$

Given an arbitrary configuration $\mathbf{x}$, for any $j$ with $x_{j}=\bar{s}$ we have a corresponding $k=\sigma j$ with $\hat{\sigma}\left(x_{k}\right)=\bar{s}$ because $x_{j}=\bar{s} \Leftrightarrow \hat{\sigma}\left(x_{\sigma j}\right)=\bar{s}$. That is, the summations in Eq. (4.6) and (4.7) are equal for any $\sigma$ for which $\omega(i, j)=\omega(\sigma i, \sigma j)$. This is true by the definition of $A_{u t}(N)$ for all permutations $\sigma \in \operatorname{Aut}_{\omega}(N)$.

\subsubsection{Single-Step Dynamics}

Proposition 4.3.1 can be applied without modification to any interacting particle system in which the local transition probabilities are a function solely of the local neighborhood configuration, as defined by an unchanging graph. ${ }^{1}$ For the class of models with single-step dynamics the proof can be done following the same argument.

\footnotetext{
${ }^{1} \mathrm{I}$ am grateful to an anonymous reviewer for this formulation.
} 
As discussed in Sec. 3.1.3, the update from one time step to the next is defined by a function $\mathbf{u}: \mathbf{S}^{r} \times \Lambda \rightarrow \mathbf{S}$ that depends on the attributes of an arbitrary number of agents $(r)$ and on an additional variable $\lambda \in \Lambda$ accounting for a possible stochastic part in the update mechanism. The probability distribution $\omega$ is therefore over $r+1$-tuples $(\omega(i, j, \ldots, k, \lambda))$. For the construction of a partition $\mathcal{M}_{\omega}$ we must now consider the group of $\sigma \in A u t_{\omega}$ with $\omega(i, j, \ldots, k, \lambda)=\omega(\sigma i, \sigma j, \ldots, \sigma k, \sigma \lambda)$. Then, as before, classes of macroscopically equivalent configurations (and therewith $\mathcal{M}_{\omega}$ ) are defined by $\mathbf{x}^{\prime}=\hat{\sigma}(\mathbf{x})$ with $\hat{\sigma}(\mathbf{x})$ as in (4.4).

\subsection{The Two-Community Model}

\subsubsection{Model}

Consider a population composed of two sub-population of size $L$ and $M$ such that $L+M=N$ and assume that individuals within the same subpopulation are connected by strong ties whereas only weak ties connect individuals that belong to different communities. We could think of that in terms of a spatial topology with the paradigmatic example of two villages with intensive interaction among people of the same village and some contact across the villages. This is similar to the most common interpretation in population genetics where this is called the island model (Wright, 1943). In another reading the model could by related to status homophily (Lazarsfeld and Merton, 1954) accounting for a situation where agents belonging to the same class (social class, race, religious community) interact more intensively than people belonging to different classes.

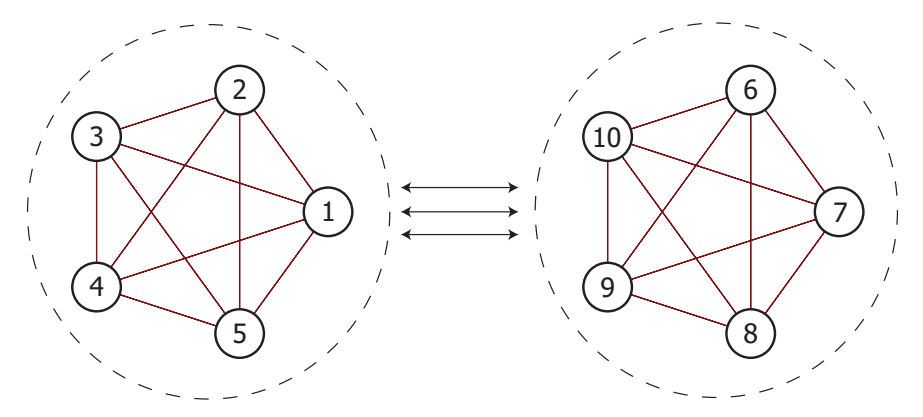

Figure 4.6: A two-component graph with two homogeneous sub-populations.

Let us adopt the perspective of a weighted graph and say that an edge with weight $a_{i j}=1$ connects agents of the same community whereas edges across the two communities have a weight $a_{i j}=r$. Therefore, $r$ is the ratio between strong and weak ties. For the VM run on such a network, notice again that there may be subtle differences in the resulting interaction probabilities $\omega(i, j)$ depending on how the agent choice is performed. First, 
in the case of link update dynamics a link $(i, j)$ is chosen out of the set of all links and so the $\omega(i, j)$ are proportional to the edge weight. Namely, let $\gamma$ denote the interaction probability between agents of the same community and $\alpha$ the respective probability across communities, then

$$
\begin{aligned}
\gamma & =\frac{1}{2 L M+((L-1) L+(M-1) M) r} \\
\alpha & =\frac{r}{2 L M+((L-1) L+(M-1) M) r},
\end{aligned}
$$

where the divisor is the sum over all edge weights and establishes that $\sum_{(i, j)} \omega(i, j)=1$. A second mode of agent choice is to first choose an agent $i$ and then choose a second agent $j$ out of its neighbor set. In the case that $M \neq L$, the interaction probabilities become different from (4.9). In the following, however, we will concentrate on the example with $M=L=50$, and in this case Eq. (4.9) gives the right interaction probabilities for node and link update dynamics.

\subsubsection{Markov Projection}

Notice, moreover, that independent of $M$ and $L$ both update modes give rise to the same symmetry group $\operatorname{Aut}_{\omega}(N)=(1 \ldots M)(M+1 \ldots N)$. $\operatorname{Aut}_{\omega}(N)$ is composed of the symmetric group $\mathcal{S}_{L}$ and $\mathcal{S}_{M}$ acting on the two subgraphs and it means that $\omega$ is invariant under permutations of agents within the same community. ${ }^{2}$ Let us denote by $m$ and $l$ the number of $\square$-agents in $M$ and $L$. It is then clear that all configurations $\mathbf{x}$ and $\mathbf{y}$ with $[m(\mathbf{x})=$ $m(\mathbf{y})] \cap[l(\mathbf{x})=l(\mathbf{y})]$ are macroscopically equivalent. As $0 \leq m \leq M$ and $0 \leq l \leq L$ the aggregation defines a Markov chain with $(M+1)(L+1)$ states which is still very small compared to the number of $2^{(M+L)}$ micro configurations. Notice that this generalizes naturally to a larger number of subgraphs. Notice also that the multipartite graphs studied in Sood and Redner (2005) fall into this category and that the authors used the respective sub-densities in their mean-field description.

The structure of the Markov chain associated to the VM on the twocommunity graph is shown in Fig. 4.7. For the system of size $M$ and $L$ the transition probabilities for the transitions leaving an atom $\tilde{X}_{m, l}$ are given by

$$
\begin{aligned}
& P\left(\tilde{X}_{m, l}, \tilde{X}_{m+1, l}\right)=\gamma(m(M-m))+\alpha(M-m) l \\
& P\left(\tilde{X}_{m, l}, \tilde{X}_{m-1, l}\right)=\gamma(m(M-m))+\alpha m(L-l) \\
& P\left(\tilde{X}_{m, l}, \tilde{X}_{m, l+1}\right)=\gamma(L-l) l+\alpha(L-l) m \\
& P\left(\tilde{X}_{m, l}, \tilde{X}_{m, l-1}\right)=\gamma(L-l) l+\alpha(M-m) l
\end{aligned}
$$

\footnotetext{
${ }^{2}$ Notice that the case $M=L$ is special because it leads to additional symmetries as the two communities are interchangeable. This is not generally the case and therefore we develop the more general case of $M \neq L$ here, even if the computations are mostly performed for the example $M=L=50$.
} 


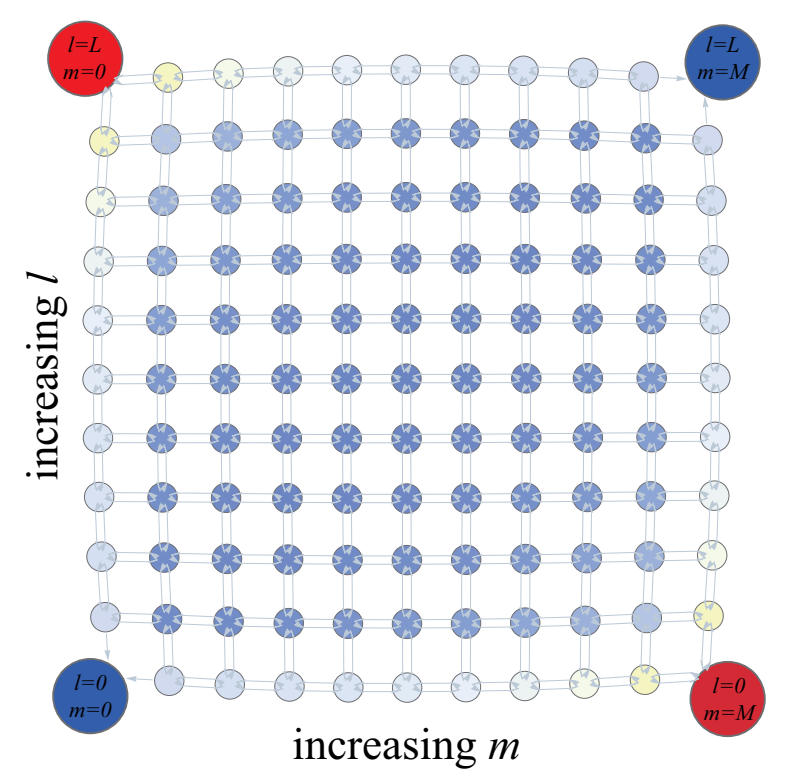

Figure 4.7: The structure of the chain for $L=M=10$. The consensus states $\tilde{X}_{0,0}, \tilde{X}_{M, L}$ as well as the states of inter-community polarization $\tilde{X}_{0, L}, \tilde{X}_{M, 0}$ are highlighted. The quasi-stationary distribution is mapped into node colors from blue (low values) to red (high values).

The four states located at the corners are highlighted in Fig. 4.7. The atom on the lower left $(l=0, m=0)$ and the upper right corner $(l=L, m=M)$ correspond to the two states of complete consensus where all agent in the two communities have adopted the same state. These are the absorbing states of the process. The other two $(l=L, m=0$ and $l=0, m=M)$ correspond to the situation that agents within the same community are aligned, but there is disagreement between the different communities. This is a form of »local alignment and global polarization«, and especially if the coupling across the communities becomes weak, there is a relatively high transient (quasi-stationary) probability for those situations. We will refer to them as inter-community polarization.

In what follows, we study a system with $M=L=50$. This gives a Markov chain of size $(M+1)(L+1)=2601$. Notice that the computations (matrix inversion and powers) needed in the analysis of that chain bear already some computational cost and that a further increase in system size will increase these costs greatly.

\subsubsection{Convergence Times}

We start the analysis of the model behavior on the two-community topology by computing the mean number of steps required to reach a final consensus 
configuration $\tilde{X}_{0,0}$ or $\tilde{X}_{M, L}$. The mean convergence times can be computed on the basis of the fundamental matrix $\mathbf{F}$ which contains the mean number of visits before absorption for all node pairs (see Sec. 3.3.2). Fig. 4.8 compares the mean convergence times for all initial states $\tilde{X}_{m, l}$ and a coupling ratio of $r=1 / 100$ (l.h.s.) to the homogeneous mixing situation with $r=1$ (r.h.s.). In comparison to the homogeneous mixing case (Eq. 3.25) the mean number of steps before absorption increases considerably for all initial configurations. For $m+l=k=50$ the complete graph will order in average after 6880 steps whereas for a weak coupling with $r=1 / 100$ this number increases to 9437 for the completely disordered configurations with $m=25, l=25$. Notably, it increases further to 11921 for the initial configurations with consensus in the communities but disagreement between the two islands (polarization).
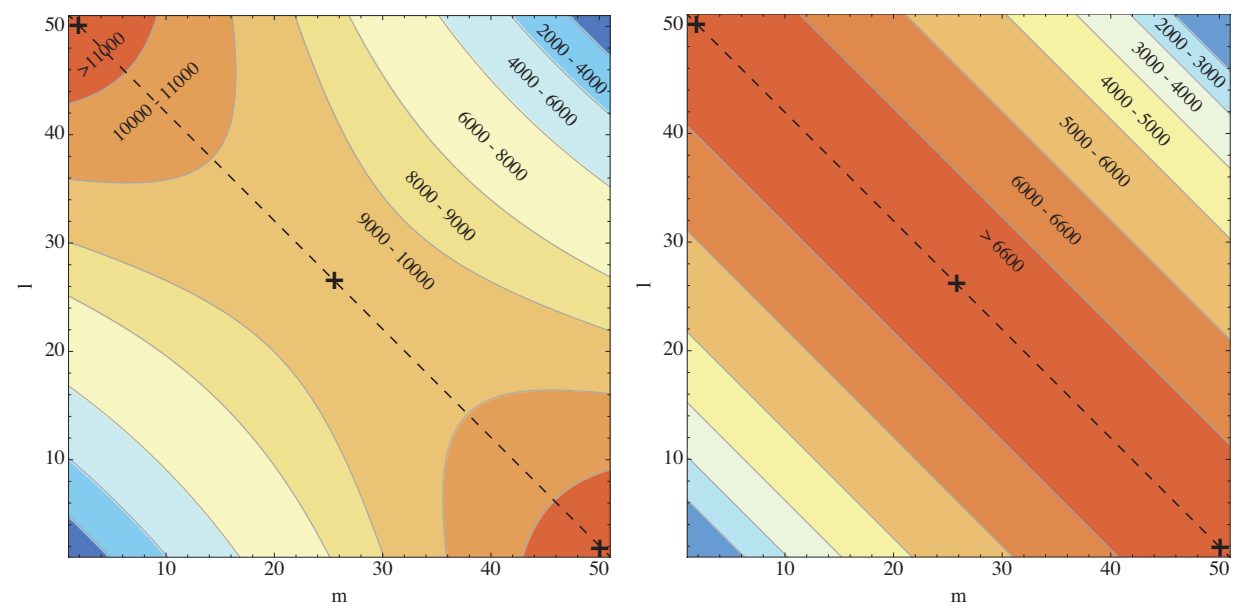

Figure 4.8: Mean convergence times for $M=L=50$ for all initial configurations $X_{m, l}$. The disordered initial configuration $X_{M / 2, L / 2}$ and the two ordered configurations $\tilde{X}_{M, 0}$ and $\tilde{X}_{0, L}$ are highlighted by + . On the l.h.s. $r=\alpha / \gamma=1 / 100$, on the r.h.s. $r=1, \alpha=\gamma$.

Notice, that in the homogeneous situation where $\alpha=\gamma$ the convergence times are only a function of the total density $k=m+l$. For every two atoms $\tilde{X}_{m_{1}, l_{1}}, \tilde{X}_{m_{2}, l_{2}}$ for which $m_{1}+l_{1}=m_{2}+l_{2}$ we obtain the same mean convergence time. This is not surprising, of course, because for $\alpha=\gamma$ the Markov chain on $\tilde{\mathbf{X}}$ is lumpable with respect to the full aggregation $\mathbf{X}=\left(X_{0}, \ldots, X_{k}, \ldots, X_{N}\right)$ and so the behavior of every $\tilde{X}_{m, l}$ within the same $X_{k}$ is identical, from the macro perspective. This changes if the VM is run on the two-community topology and the coupling between the communities is smaller than the coupling among agents in the same island (i.e., $\alpha<\gamma)$. Due to the topological effects and a reduced communication across communities, the emergence of a meta-stable configuration of local alignment within communities but global polarization across communities is likely. In 
general, the process requires more time to converge if initialized in a situation where one community is ordered from the beginning and the opinions diverging from that consensus concentrate in the other community. Notably, an increase in convergence times is observed even for the initial configurations which are completely disordered (e.g., $\tilde{X}_{25,25}$ ), because a considerable number of realizations is first driven to a state of inter-community polarization before it eventually evolves further to a consensus profile (see below).

We compare these two situations (namely initial disorder $\tilde{X}_{25,25}$ and initial order $\left.\tilde{X}_{50,0}\right)$ by considering the distribution of convergence times for two configurations with $m+l=N / 2=50$. The respective cumulative distributions for $r=1 / 100$ is shown on the l.h.s. of Fig. 4.9 and on the r.h.s. the respective probability of absorbency at time $t$ is shown.
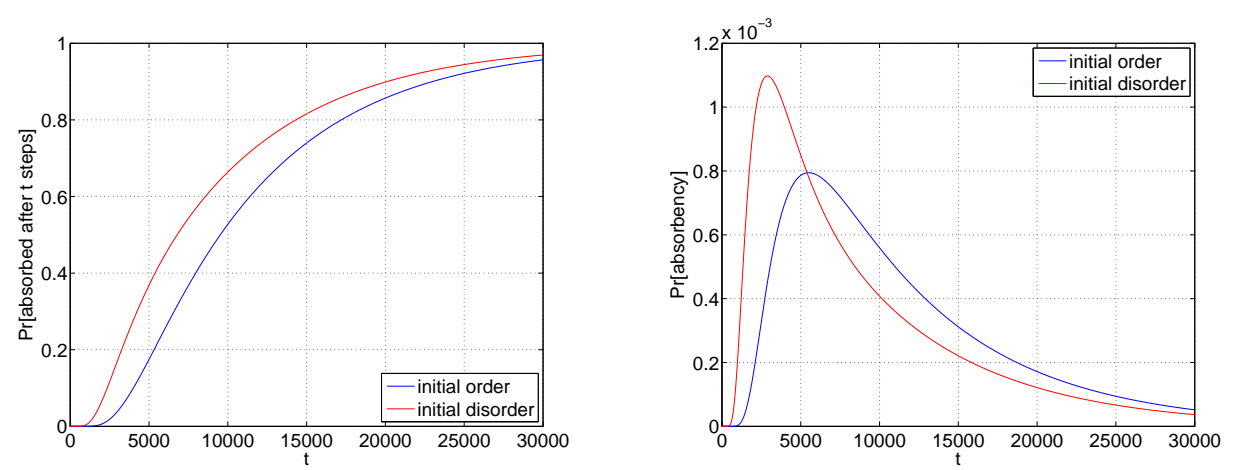

Figure 4.9: Distribution of convergence times $\tau$ for $M=L=50, r=$ $1 / 100$ for the disordered initial configuration $X_{M / 2, L / 2}$ and the two ordered configurations $\tilde{X}_{M, 0}$ and $\tilde{X}_{0, L}$ with inter-community polarization.

In the case of initial disorder (red curve), where the states $\square$ and are distributed equally over the two islands, there is a certain number of realizations that approaches one absorbing consensus state without entering the states of partial order $\left(\tilde{X}_{M, 0}\right.$ and $\left.\tilde{X}_{0, L}\right)$. The probability of absorbency reaches a peak after a relatively short time of around $t \approx 3000$ steps whereas the highest absorbency probability lies around $t \approx 5000$ for the ordered initial condition. At around $t \approx 5000$ already $40 \%$ of realizations have converged for the disordered case, but only $20 \%$ in case of initial order. This shows that there is a strong influence of the interaction topology leading to a high heterogeneity between different initial configurations with the same global magnetization $k=m+l$. The ordered configurations $\tilde{X}_{M, 0}$ and $\tilde{X}_{0, L}$ function as dynamical traps and it may take a long time to escape from them especially when $r$ becomes small. On the other hand, however, Markov chain theory tells us that the probability for very long waiting times decays exponentially.

In Fig. 4.10, a more detailed picture of how convergence times increases as $r=\alpha / \gamma$ decreases is provided. For the two initial situations considered 


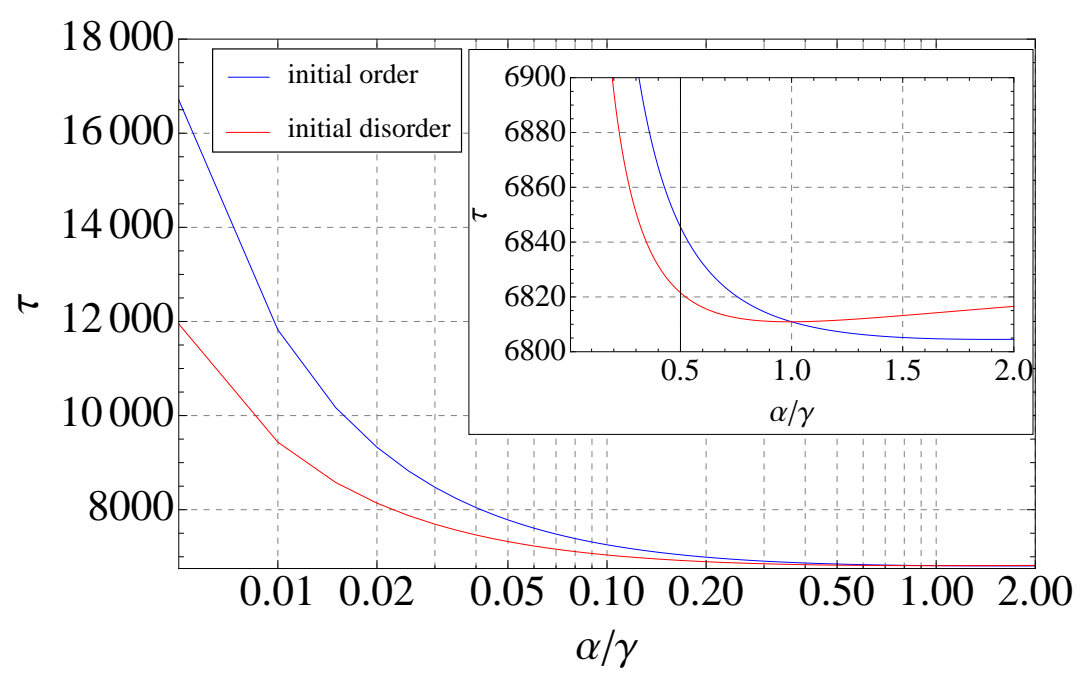

Figure 4.10: Mean convergence times $\tau$ as a function of the relative intergroup interaction strength $r=\alpha / \gamma$ for the ordered initial configuration $\tilde{X}_{M, 0}$ and the disordered initial configuration $\tilde{X}_{M / 2, L / 2}$.

previously the mean convergence times are shown as a function of $r=\alpha / \gamma$. Notice again that these extreme configuration are highlighted by + in Fig. 4.8. It is clear that the mean times to absorbency diverge as $r$ approaches zero, $\lim _{r \rightarrow 0} \tau=\infty$. This is due to the fact that the interaction topology becomes disconnected in that extreme case, and therefore, the non-consensus configurations $\tilde{X}_{M, 0}$ and $\tilde{X}_{0, L}$ become absorbing. In other words, to go from (say) $\tilde{X}_{0, L}$ to (say) $\tilde{X}_{0,0}$ requires an infinite number of steps. In fact, we then deal with a completely new chain that has four absorbing states, or more precisely, with two chains one for each island. However, as long as $r>0$ the possibility to escape from $\tilde{X}_{0, L}$ remains, even if it takes very long.

\subsubsection{Quasi-Stationary Distribution}

Finally, to characterize the long-term transient behavior, let us look at the quasi-stationary distribution of the two-community VM. This distribution contains the probabilities to be in the different transient states for realizations that are not absorbed after a certain time. It corresponds the normalized left eigenvector associated to the largest eigenvalue of the transient sub-matrix $Q$ of $P$ (just as the stationary distribution of a regular chain is the normalized left eigenvector of the transition matrix $P$ ). See, for instance, Darroch and Seneta (1965) (pages 91 - 93 in particular) for a description of the quasi-stationary distribution.

Fig. 4.11 shows the quasi-stationary distribution for the two-community 

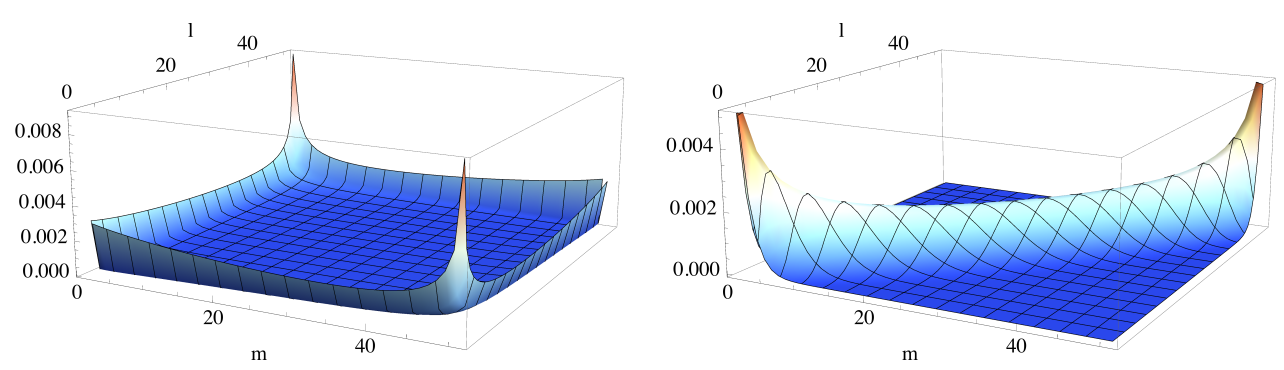

Figure 4.11: Quasi-stationary distribution for the VM on two islands with $r=1 / 100$ (l.h.s.) and $r=1$ (r.h.s.).

VM with $r=1 / 100$ and $r=1$. Notice again that the later corresponds to the homogeneous mixing case. If $r$ is small there is a high (conditional) probability that the process is trapped in one of the states of inter-community polarization. Also the states $\tilde{X}_{m, 0}$ and $\tilde{X}_{0, l}$ with one uniform sub-population have a relatively high probability indicating that convergence to complete consensus out of local order does not happen via a transition through complete disorder. This is in stark contrast to the homogeneous mixing situation, which is shown on the r.h.s. of Fig. 4.11. In this case, states of intercommunity polarization $(m=M, l=0$ and $m=0, l=L)$ and states close to that become in effect very rare random events. ${ }^{3}$

\subsection{On the Role of Peer-to-Peer Communication in an Opinion Model with Leader}

This section presents a Markov chain treatment of the VM on a topology that models leadership. We show how the probability that the leader imposes its opinion on a follower population increases with the influence asymmetry between a leader and the followers and is independent of peer-to-peer processes among followers. A greater influence does not only increase the respective exit probability, it also accelerates the convergence process. However, this acceleration is undermined by a stronger peer-to-peer communication among followers.

\subsubsection{Model}

Here we study the binary VM on an asymmetric topology. Namely, we introduce an opinion leader that has an increased influence on the rest of the

\footnotetext{
${ }^{3}$ The reason for this is clear. The number of micro configurations $x \in \boldsymbol{\Sigma}$ mapped into the state $\tilde{X}_{m, l}$ is $\left(\begin{array}{c}M \\ m\end{array}\right)\left(\begin{array}{c}L \\ l\end{array}\right)$ which is a huge number for $m \approx M / 2, l \approx L / 2$ but only 1 for $m=M, l=0$ and $m=0, l=L$. Because under homogeneous mixing there is no favoring of particular agent configurations with the same $k=m+l$ the stationary probability at macro scale is proportional to the cardinality of the set $\tilde{X}_{m, l}$.
} 
agent population. Therefore, consider a homogeneous population of $N$ agents indexed by $i=1, \ldots, N$. As before, each agent can adopt two opinions, or $\mathbf{\square}$. Consider further that there is another agent (the leader indexed by $i=0$ ) with a stronger influence on the population such that the probability that its attribute spreads in the population is increased. In principle, we also want to allow that the population influences the leader, but the probability of such an event is rather small.

The VM operates by choosing an agent $(i)$ which adopts the opinion of one of its neighbors $(j)$. As before, $\omega(i, j)$ is the probability that the pair $(i, j)$ is chosen. Under link update, and with the convention that the first agent $(i)$ imitates the second $(j)$, leadership can be included by introducing an asymmetry in the interaction probabilities such that it is more probable to choose the leader in second place. Then, it becomes more probable that its state is adopted by another agent. We index the leader with a 0 and assign the following interaction probabilities:

$$
\begin{gathered}
\omega(0, j)=\beta, \\
\omega(i, 0)=\alpha, \\
\omega(i, j)=\gamma,
\end{gathered}
$$

$i, j=1, \ldots, N$. An increased probability that the leader opinion is adopted by a follower is modeled by $\alpha \gg \beta$. The third probability, $\gamma$, accounts for the probability of a peer-to-peer interaction which does not involve the leader. Notice that with Eqs. (4.10) the model is formulated directly in terms of interaction probabilities $\omega$ by which we avoid a possible confusion between link and node update dynamics (Sec. 4.2). The resulting interaction topology is shown in Fig. 4.12.

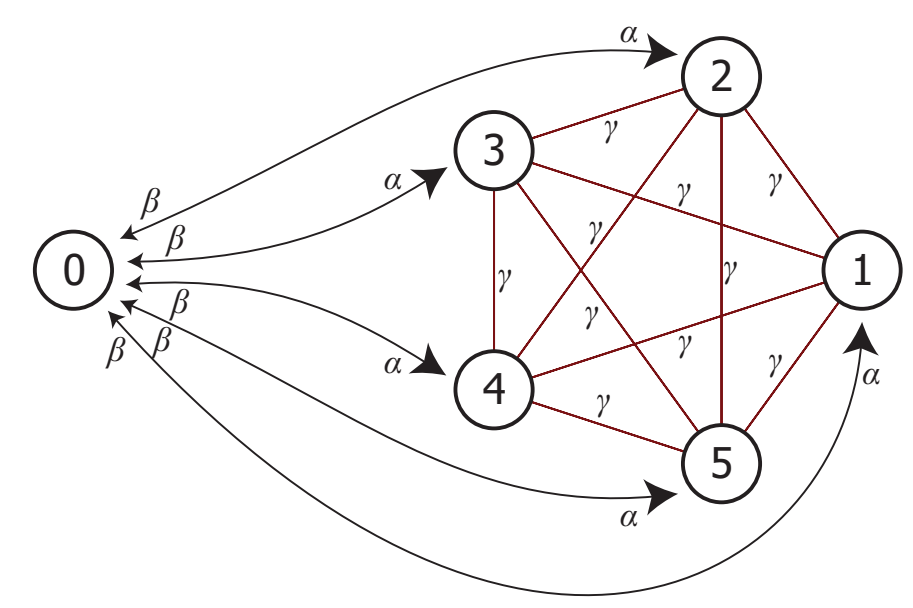

Figure 4.12: Agent interaction topology in which one agent (indexed by 0 ) has an increased influence on the rest of the population. 


\subsubsection{Markov Projection}

It is clear that $\omega$ is still highly symmetric, because $\omega(i, j)=\gamma(4.10)$ for all agents in the follower population. More precisely, $\omega$ is invariant under all permutations $\mathcal{S}_{N}$ of the $N$ agents in the follower population and therefore the topology shown in Fig. 4.12 is topologically equivalent to the star graph of size $N+1$ (in fact, with $\gamma=0$ it is a star). Notice, moreover, that the leader topology is actually a special case of the two-community structure obtained by setting $M=1$ and $L=N$.

Namely, the topology shown in Fig. 4.12 gives rise to the symmetry group $A u t_{\omega}=(0)(1 \ldots N)$. In this case, a lumpable partition is obtained by the independent observation of the state of the leader $x_{0}$ and the number of follower agents in the different states (given by $f=N_{\square}\left(\mathbf{x} / x_{0}\right)$ ). This partition is given by $\tilde{\mathbf{X}}=\left\{\tilde{X}_{l, f}: l=0,1 ; f=0, \ldots, N\right\}$ with

$$
\tilde{X}_{l, f}=\left\{\mathbf{x}: N_{\square}\left(x_{0}\right)=l \cap N_{\square}\left(\mathbf{x} / x_{0}\right)=f\right\} .
$$

In words, the subsets $\tilde{X}_{0, f}$ contain all configurations $\mathbf{x} \in \boldsymbol{\Sigma}$ in which the leader is in state and f follower agents are in state $\square$. Respectively, the subsets $\tilde{X}_{1, f}$ contain the configurations with $x_{0}=\square$ and $f$ follower agents in state $\square$. It is thus clear that for the leader-follower system with total size of $N+1$ the macro chain has $2(N+1)$ states. The associated chain along with the corresponding transition probabilities is shown in Fig. 4.13.

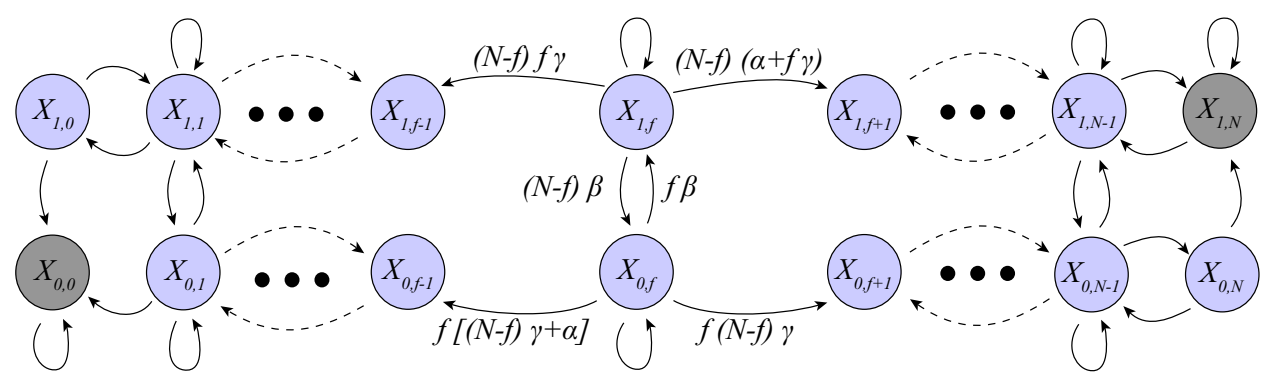

Figure 4.13: The macro chain associated with the binary VM on the leaderfollower topology. The transition probabilities are also shown.

In principle, all the information about the convergence probabilities and the transient behavior can be obtained by computing the fundamental matrix of that system (Kemeny and Snell, 1976, Chapter 3). For the leader-follower system the transition matrix $P$ is a $2(N+1) \times 2(N+1)$ dimensional matrix and the matrices $Q$ and $\mathbf{F}=(\mathbf{1}-Q)^{-1}$ are of size $2 N \times 2 N$. Hence, the computation of the fundamental matrix requires the inversion of a matrix of that size. At least numerically, this is doable on an up-to-date computer platform for systems of several thousand agents. 


\subsubsection{Simple Observations}

However, let us begin the analysis with two simple observations. First of all, it is clear from Fig. 4.13 and the transition probabilities that the macro chain has two absorbing states corresponding to the uniformity configurations $\tilde{X}_{1, N} \equiv(\square \square \ldots \square)$ and $\tilde{X}_{0,0} \equiv$

Secondly, notice that in case $\beta=0$ - the case that followers have no influence on the leader at all - there is a zero probability that the leader changes its state. This means that the macro chain is disconnected and the model performs a random walk on the upper or the lower chain depending on the initial state of the leader (see Fig. 4.13). It is clear, then, that the process converges to the configuration in which all follower agents adopted the state of the leader.

\subsubsection{Influence of the Leader}

How does an increasing asymmetry $(\alpha>\beta)$ effect the model behavior? To address this question we compute the probability that the system converges to the initial state of the leader. Consider the opinion leader is in state $\square$ at $t=0$. The probability that all agents end up in $\square$ as $t \rightarrow \infty$ is

$$
\operatorname{Pr}_{t \rightarrow \infty}\left[\tilde{X}_{1, f} \rightarrow \tilde{X}_{1, N}\right]=\frac{\alpha+f \beta}{\alpha+N \beta} .
$$

As the ratio $\frac{\alpha}{\beta}$ increases, the chances that the system converges to the leader's opinion increase very fast in the beginning, and approach 1 in the limit $\frac{\alpha}{\beta} \rightarrow \infty$. Notice that even in the case that all followers are against the opinion of the leader, an influence ratio of $\frac{\alpha}{\beta}>N$ is sufficient to obtain a chance of $\operatorname{Pr}_{t \rightarrow \infty}\left[\tilde{X}_{1,0} \rightarrow \tilde{X}_{1, N}\right]>1 / 2$ that the leader imposes its opinion against the consensus opinion in the follower population. More precisely, for $\frac{\alpha}{\beta}=N$, Eq. (4.12) becomes $\operatorname{Pr}_{t \rightarrow \infty}\left[\tilde{X}_{1,0} \rightarrow \tilde{X}_{1, N}\right]=\frac{1}{2}+\frac{f}{N}$. If the leader has such a strong influence, convergence to its state hence becomes the most probable option.

Fig. 4.14 shows this probability as a function of the ratio $\frac{\alpha}{\beta}$ for different initial proportions $f$ of follower agents in the same state as the leader. We set $N=100$ in Eq. (4.12) in which case $f=N / 2=50$ corresponds to the case that the followers are divided into two groups of equal size.

It is noteworthy, that the exit probabilities do not depend on the strength of the peer-to-peer interaction $\gamma$. Therefore the influence of the opinion leader is as if there was no communication at all among the followers. At a first sight this seems a bit counter-intuitive, but considering that $\gamma$ does not introduce any bias in favor of one or the other consensus state it is not too surprising (see Fig. 4.13).

Finally, we see from Eq. (4.12) that the case $\alpha=\beta$ restores the results obtained in the VM with homogeneous mixing (and in general for the VM 


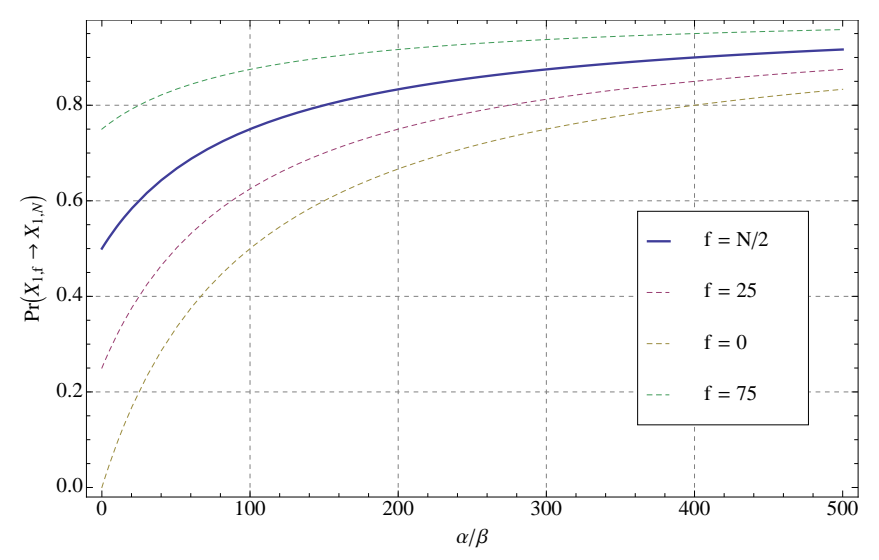

Figure 4.14: The probability that the leader imposes its opinion onto the entire population as a function of $\frac{\alpha}{\beta}$ for different initial proportions $f$ of follower agents in the leader's state.

on undirected networks). For the configurations with the leader in $\square$ we obtain for $\operatorname{Pr}_{t \rightarrow \infty}\left[\tilde{X}_{1,0} \rightarrow \tilde{X}_{1, N}\right]=\frac{f+1}{N+1}$ where $f+1$ is just the total number of individuals in state $\square$ and $N+1$ the total number of agents.

\subsubsection{Convergence Times}

We now look at the mean convergence times as a function of the network parameters $\alpha, \beta$ and $\gamma$ for a finite system of $N=100$ followers and one leader. Because $\omega$ is a distribution over all agent pairs, we have $N[\alpha+\beta+(N-1) \gamma]=$ 1 . This means that there are effectively two free parameters in that analysis and it is convenient to study the network influence in term of the ratios $\alpha / \beta$ and $\gamma / \beta$. To obtain the mean convergence time, the fundamental matrix is computed for different ratios $\alpha / \beta=1,2, \ldots 50$ and $\gamma / \beta=1,2, \ldots 50$ from which the respective mean convergence times can be obtained directly (Kemeny and Snell, 1976, 49-51). Notice that with any relative increase of $\alpha / \beta$ and $\gamma / \beta$ the probability $\beta$ decreases and this means that a leader change becomes less likely. Increasing $\alpha / \beta$ corresponds to an increasing asymmetry between leader and followers, an increase in $\gamma / \beta$ to an intensification of the (symmetric) mutual influence in the follower population.

The result of this analysis is shown in form of a contour plot in Fig. 4.15. We notice two basic opposing trends in that plot. First, a stronger influence of the leader (increasing $\alpha / \beta$ ) tremendously speeds up the convergence to one of the absorbing consensus states. The leader strongly drives the entire system towards its initial state and with a relatively high probability consensus is reached without a state change of the leader. On the other hand, however, an increasing mutual influence among the followers (increasing $\gamma / \beta$ ) may rule out this effect and slow down the process so that convergence to 


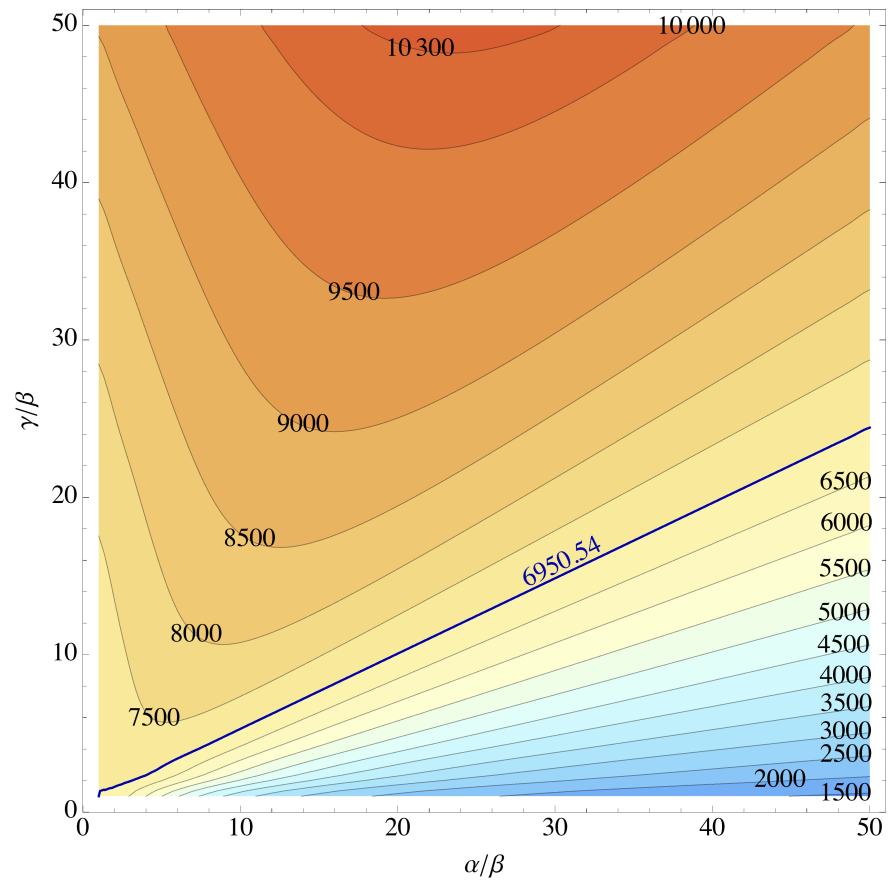

Figure 4.15: Mean convergence times for $\alpha / \beta=1 \ldots 50$ and $\gamma / \beta=1 \ldots 50$. The thick blue line indicates the convergence time for the VM with homogeneous mixing.

a final consensus state takes more time. Noteworthy, in a large area of the parameter space it takes even more time than the VM with homogeneous mixing of the same size (obtained with $\alpha=\beta=\gamma$ ). This is illustrated by the thicker blue contour line. For all parameter configurations above this line, mean convergence times become larger compared to the homogeneous mixing case.

Let us consider two examples. First, the case $\alpha / \beta=100$ and $\gamma / \beta=1$ in a system of $N=100$ followers with an initial number of $N / 2=50$ followers and the leader in $\square$. With this parameter constellation, the mean number of steps until convergence is (only) 887 steps. It is easy to compute that in this relatively short period, approximately $60 \%$ of the realizations have been absorbed, $50 \%$ in $\tilde{X}_{1, N}$ and $10 \%$ in $\tilde{X}_{0,0}$ (the latter involving at least one change of the leader). Virtually all remaining $40 \%$ of realizations are in fact very close to absorbency: in $\approx 33.5 \%$ of the cases more than $90 \%$ of followers are in the state of the leader.

While a stronger mutual influence among the followers does not affect the overall exit probabilities (see above), it may rule out the acceleration of convergence to the state a strong leader. Consider, as another extreme example, the case $\alpha / \beta=100$ and $\gamma / \beta=100$ in a system of $N=100$ followers with an initial number of $N / 2=50$ followers and the leader in $\square$. 
This leads to an average convergence time of 9278 steps and to a long-term transient behavior in which all follower constellations are equally likely (see below, Fig. 4.16).

\subsubsection{Transient Behavior}

In order to obtain a complete picture of the transient behavior of the model, we first compute the quasi-stationary distribution for the two examples. The respective probability to observe $f$ followers in state $\square$ in the long run is shown in Fig. 4.16. The blue curve represents the case of a strong leader

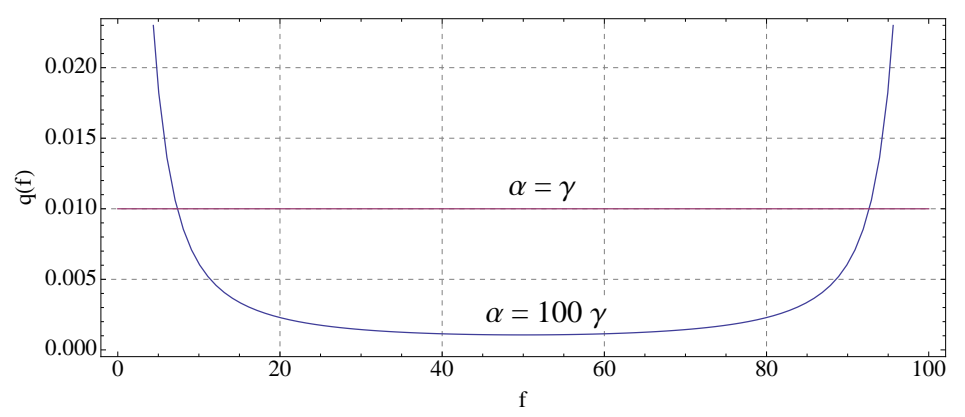

Figure 4.16: The probability to observe $f$ followers in the long run for the two examples computed using the quasi-stationary distribution.

$(\alpha / \beta=100$ and $\gamma / \beta=1)$. Eq. (4.10) tells us that in this case, an agent pair $(i, 0)$ (follower, leader) will be chosen in half of the cases (as $N \alpha=1 / 2$ ), the probability for the choice of two follower agents is also close to one half, namely $N(N-1) \gamma=99 / 200$. Therefore, a constellation $(0, j)$ (leader, follower) is chosen in average only once in 200 time steps. Notice, moreover, that a state change really takes place only if $x_{0} \neq x_{i}$ and that therefore the change of the leader becomes even more unlikely because the leader has already imposed its opinion on most of the followers. Therefore, even if the parameter constellation allows that followers change the state of the leader $(\beta>0)$, a persistent situation in which the follower population opposes the leader or at least remains close to the fifty-fifty configuration cannot be observed. Once the leader changes its state, it quite immediately drives the population of followers to the opposite extreme corresponding to its opinion.

The most likely behavior of the model with strong leader is also highlighted by the mean hitting times shown in Fig. 4.17. It shows the expected number of steps a process starting in $\tilde{X}_{1,50}$ is in the different atoms $\tilde{X}_{1, f}$ (white circles for $x_{0}=\square$ ) and $\tilde{X}_{0, f}$ (dark circles for $x_{0}=\mathbf{\square}$ ). Notice that the initial state $\tilde{X}_{1,50}$ corresponds to the light circle at $f=50$. In order to approach the more probable absorbing state $\tilde{X}_{1, N}$ the process has to transit through all the states to the right with $f>50$, and consequently the mean hitting times of these states are high compared to the rest. If, on that way, a 


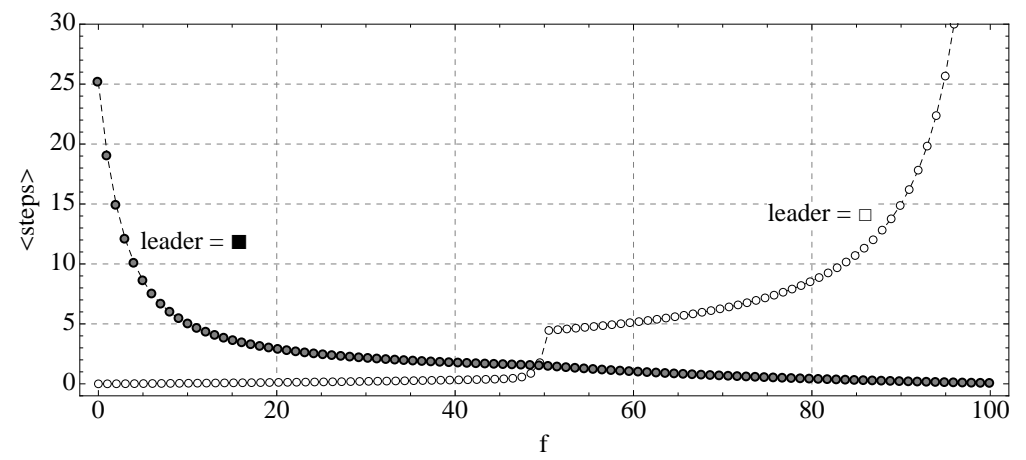

Figure 4.17: Mean number of steps a process starting in $\tilde{X}_{1,50}$ is in the different atoms $\tilde{X}_{1, f}$ (light) and $\tilde{X}_{0, f}$ (dark) for $\alpha / \beta=100$ and $\gamma / \beta=1$.

state change of the leader takes place, the process goes to the other extreme passing through the transient states with intermediate $f$ rather rapidly. Finally, the drop off in the hitting time to the left of the initial state gives an idea of how strong the leader influence is in this case. There is virtually a zero probability that social influence processes among the followers drive the system far from the leader state and, in fact, at least in a small system of 100 followers a second state change of the leader is quite rare.

In the second example with $\alpha / \beta=100$ and $\gamma / \beta=100$ the model behaves in a different way. First of all, the quasi-stationary distribution shown in Fig. 4.16 (red curve) tells us that, in the long run, all follower constellations are equally likely. This decoupling from the leader is surprising if we recall that the exit probabilities are strongly biased in favor of the initial state of the leader (see Eq. (4.12) and Fig. 4.12) and are not affected by the strong peer-to-peer interaction.

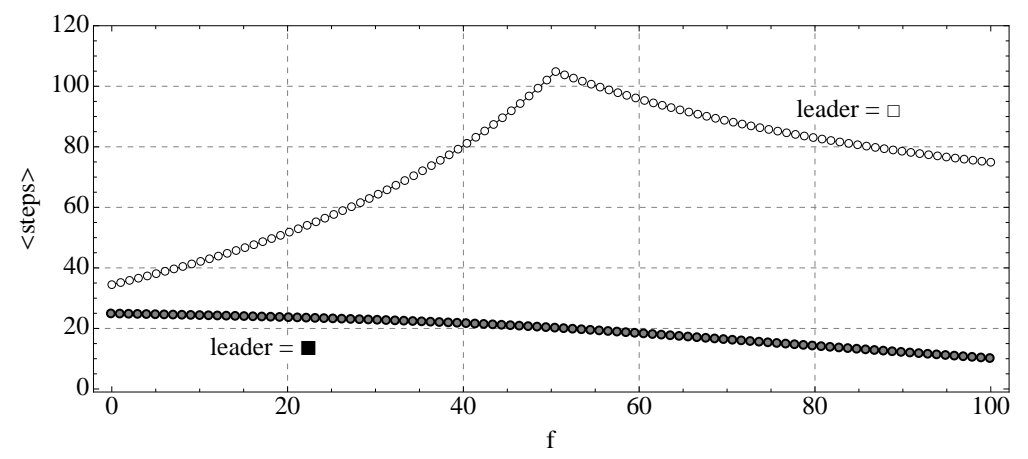

Figure 4.18: Mean number of steps a process starting in $\tilde{X}_{1,50}$ is in the different atoms $\tilde{X}_{1, f}$ (light) and $\tilde{X}_{0, f}$ (dark) for $\alpha / \beta=100$ and $\gamma / \beta=100$.

The mean hitting times for that example are shown in Fig. 4.18. It becomes clear that the behavior strongly resembles the behavior of the VM 
with homogeneous mixing (see Fig. 3.8, previous chapter). But despite these similarities in mixing behavior the exit probabilities remain strongly biased for $\alpha / \beta=100$. The main reason for the difference in the transient behavior is that the effective influence rate of the leader is reduced tremendously as $\gamma$ approaches $\alpha$. By Eq. (4.10) we see that the probability of choosing an agent pair $(i, 0)$ (follower, leader) becomes in fact very small now (as $N \alpha=100 / 10001 \approx 1 \%$ ), the probability for the choice of two follower agents instead effectively increases to $N(N-1) \gamma=9900 / 10001 \approx 98.99 \%$. Consequently, a constellation $(0, j)$ (leader, follower) in which the leader could change its state is chosen in average only once in 10000 time steps.

\subsubsection{Alternative Interpretation}

In fact, the second example with $\alpha / \beta=100$ and $\gamma / \beta=100$ (and consequently $\alpha=\gamma$ ) calls for an interpretation not in terms of leader and followers, but suggests to understand the $\gg$ leader « as a member of the group that is just not so amenable to influence, compared to the others. Namely, $\omega(i, 0)=\alpha=\gamma=\omega(i, j)$ means that followers give the same importance to the leader as to any other follower agent. On the other hand, $\beta$ becomes very small so that the chances that followers change the leader are reduced. Of course, such a situation may appear only in a small system, but not at the scale of populations. It may happen at the group level of (say) 20 individuals (of course, only in an approximate sense). In this context, the analysis shows that a single stubborn individual can strongly influence the outcome of a consensus process (in small groups).

\subsection{The Ring}

Prop. 4.3.1 generalizes to networks with arbitrary automorphisms which we illustrate at the example of the ring graph. When the model on the ring with nearest neighbor interactions is defined by $\omega(i, i+1)=\frac{1}{N}: i \bmod N$, it possesses an invariance with respect to translations. That is, the automorphism group $\operatorname{Aut}_{\omega}(N)$ consists of all cyclic shifts of agents generated by $\sigma:(1,2, \ldots, N) \rightarrow(N, 1,2, \ldots, N-1)$. Notice that translational symmetries of this kind also play an important role in the determination of the relevant dimensions of spin rings Bärwinkel et al. (2000) and that there are interesting parallels in between the two problems.

Consider a ring of five agents $(N=5)$ with $2^{5}=32$ micro states. For $\mathbf{x}=$ (घ口) it is clear that $\sigma^{k}(\mathbf{x})=\mathbf{x}$ for all $k$. That is, $\mathbf{x}=(\mathbf{\square} \mathbf{\square})$ with $k=0$ constitutes a class of its own. For $k=1$, we may use $x_{1}=(\square \square \square \square)$ as a generator (4.5) for its class. As all 5 configurations with $k=1$ can be obtained shifting $x_{1}$, all of them are in the same equivalence class. The 10 configurations with $k=2$ cannot be lumped into the same macro state. There are two classes differentiated by the distance of zero or one in between 

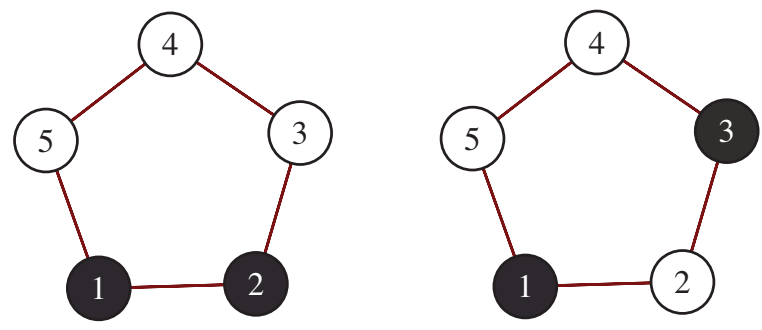

Figure 4.19: Two configurations with equal $k=2$ which are not macroscopically equivalent for the ring with $N=5$.

the two black agents (see Fig. 4.19). Using the two configurations shown in Fig. 4.19 as generators yields two equivalence classes each containing five micro states. The cases $k=3,4,5$ follow by symmetry so that all in all the dimension $\tilde{\mathbf{X}}$ of macro chain is reduced to 8 .

In the general case of $N$ agents we can in principle proceed in the same way. However, the number of macro states will increase considerably with the system size. We finish this section with a quantification of this number for the ring for which we can use a well-known enumeration theorem due to Pólya (see Harary and Palmer (1973), 35-45, Eqs.(2.2.10) and (2.4.15) in particular). According to this, the number of macro states is

$$
|\tilde{\mathbf{X}}|=\frac{1}{N} \sum_{k \mid N} \varphi(k) 2^{\frac{N}{k}}
$$

where $\varphi(k)$ is the Euler $\varphi$-function and the sum is over the divisors $k \mid N$ of $N$. As an approximation we have $|\tilde{\mathbf{X}}| \approx 2^{N} / N$. Hence, an explicit solution of the macro chain will be possible only for very small systems.

\subsection{Discussion}

We have seen in the previous chapter that the full aggregation illustrated in Figs. 3.3 and 4.1 is lumpable only if the interaction probabilities are uniform. This corresponds to the VM implemented on the complete graph in which $\omega(i, j)=1 / N(N-1)$ (or $1 / N^{2}$ if self-choice is allowed). It is, of course, wellknown that the macro model obtained in terms of $h(\mathbf{x})=k$ fully describes the evolution of the micro model on the complete graph, but not on other topologies, see (Slanina and Lavicka, 2003, 3) and (Castellano et al., 2009, 601). Nevertheless, Proposition 4.3.1 sheds light on the (probabilistic) reason for this. Namely, the complete graph and respectively homogeneous mixing is the only topology for which the automorphism group is the group $\mathcal{S}_{N}$ of all permutations of $N$ agents. In this case, for any two configurations $\mathbf{x}, \mathbf{x}^{\prime}$ with an equivalent aggregate $k$ there is a $\sigma \in \mathcal{S}_{N}$ such that $\mathbf{x}=\sigma\left(\mathbf{x}^{\prime}\right)$. Hence, an equivalent aggregate value $k$ implies macroscopic equivalence. The fact that 
this is only true for complete graph and homogeneous mixing underlines how restrictive these conditions are.

The more complex the internal structure of the agents and the more heterogeneous their interaction behavior, the lower our chances to derive a loss-less coarse-graining that leads to a tractable Markov chain. It is clear that in heterogeneous networks with a small number of automorphisms the coarse-graining is limited because only a few micro states are macroscopically equivalent and can be lumped. As this method is based on exact graph automorphisms it is more suited for stylized situations as the two-community and the leadership model discussed in Secs. 4.4 and 4.5.

On the other hand, the method informs us in this way about the complexity of a system introduced by non-trivial interaction relations. Even in a model as simple as the VM, the behavior of whole system is not completely described by summation over its elements (full aggregation in terms of $k$ ), because non-trivial dynamical and spatial effects may emerge at the macro level. In this sense, our work is related to key concepts in the area of computational emergence (Bedau, 2003; Huneman and Humphreys, 2008) dealing with criteria and proper definitions of emergence. Thereafter $\gg$ an emergent phenomenon is one that arises from a computationally incompressible process « (Huneman and Humphreys, 2008, 425/26). Markov projections as discussed here in the context of the VM provide explicit knowledge about the (in)compressibility of computational models and may therefore help to operationalize these rather abstract definitions. This issue is further discussed in Chapter 6.

Let us finally note that in general there may be many partitions $\mathcal{M}$ of the state space that are lumpable and here no statement is made here about optimality of the partition $\mathcal{M}_{\omega}$ generated by the application of Prop. 4.3.1. On the other hand, a simple answer is provided by a closer inspection of the VM with homogeneous mixing telling us that $\mathcal{M}_{\omega}$ is not optimal in that case. Namely, we have for any $k, P\left(X_{k}, X_{k \pm 1}\right)=P\left(X_{(N-k)}, X_{(N-k) \mp 1}\right)$ which means that the pairs $\left\{X_{k}, X_{(N-k)}\right\}$ can be lumped into the same state. The reason for this is that the VM update rule brings about an additional symmetry that is not accounted for in $A u t_{\omega}$ and therefore not in $\mathcal{M}_{\omega}$. More generally, the micro structure of the VM is always symmetric with respect to the simultaneous flip of all agent states $x_{i} \rightarrow \bar{x}_{i}, \forall i$ and therefore, independent of the interaction topology, $\hat{P}(\mathbf{x}, \mathbf{y})=\hat{P}(\overline{\mathbf{x}}, \overline{\mathbf{y}})$. 


\section{NETWORK EFFECTS IN THE CONTRARian Voter MOdel}

The objective of this chapter is two-fold. On the one hand, it presents an application of the theory to the contrarian voter model (CVM) including a Markov chain solution for homogeneous mixing and the two-community model. On the other hand, this chapter presents a first step to study how microscopic heterogeneity may lead to macroscopic complexity when the aggregation procedure defines a non-Markovian macro process.

Sec. 5.1 introduces the CVM and derives the corresponding microscopic Markov chain. Sec. 5.2 deals with the model on the complete and the twocommunity graph with a particular focus on the stationary dynamics of the model. The model dynamics are studies in terms of the contrarian rate $p$ and the coupling $r$ between the two-communities. After the discussion of the two stylized topologies, Sec. 5.3 shows the effect of various paradigmatic networks on the macroscopic stationary behavior. In Sec. 5.4 we return to the two-community CVM and find with it an analytical scenario to study the discrepancy between a mean-field model (homogeneous mixing) and the model on a more complex (though still very simple) topology. It shows that memory effects are introduced at the macro level when we aggregate over agent attributes without sensitivity to the microscopic details.

Some parts of this chapter have been presented at the ECCS 2013 satellite on cultural and opinion dynamics (Banisch, 2013) and will be submitted to an associated special issue in Advances in Complex Systems.

\subsection{The Contrarian Voter Model}

In order to address questions related to the macro effects of heterogeneous interaction probabilities we will concentrate on an extension of the VM called contrarian voter model (CVM) or sometimes anti-voter model. Contrarian behavior relates to the presence of individuals that do not seek conformity under all circumstances or to the existence of certain situations in which 
agents would not desire to adopt the behavior or attitude of their interaction partner. Contrarian behavior has been addressed previously, see, for instance, Galam (2004); Li et al. (2012).

\subsubsection{Model}

As the VM, which has been discussed at length in the previous chapters, the CVM is a binary opinion model where $N$ agents can adopt two different opinions: $\square$ and $\boldsymbol{\square}$. The model is an extension of the VM in order to include a form of contrarian behavior. At each step, an agent $(i)$ is chosen at random along with one of its neighbors $(j)$. Usually (with probability $1-p$ ), $i$ imitates $j$, but there is also a small probability $p$ that agent $i$ will do the opposite (contrarian extension). More specifically, if $i$ holds opinion and meets an agent $j$ in $\square, i$ will change to $\square$ with probability $1-p$, and will maintain its current state with probability $p$. Likewise, if $i$ and $j$ are in the same state, $i$ will flip to the opposite state with probability $p$.

While the VM rule may be interpreted as a kind of ferromagnetic coupling by which neighboring spins (agents) align, the contrarian rule can be interpreted as anti-ferromagnetic coupling by which neighbors are of opposed sign after the interaction. Table 5.1 illustrates the update rules for the CVM.

\begin{tabular}{|c|c|c|c|c|c|c|c|c|}
\hline Prob. & $x_{j}$ & $x_{j}$ & $(1-p)$ & $\square$ & $\square$ & $p$ & D & $\square$ \\
\hline$x_{i}$ & $y_{i}$ & $y_{i}$ & $\square$ & 口 & $\square$ & 口 & $\square$ & 口 \\
\hline$x_{i}$ & $y_{i}$ & $y_{i}$ & $\square$ & $\square$ & $\square$ & $\square$ & $\square$ & च \\
\hline
\end{tabular}

Table 5.1: Update rules $y_{i}=\mathbf{u}\left(x_{i}, x_{j}\right)$ for the CVM. The VM rule (ferromagnetic coupling) is applied with probability $(1-p)$, the contrarian rule (anti-ferromagnetic coupling) with probability $p$.

\subsubsection{Micro Dynamics}

From the micro-level perspective (see Sec. 3.1), the CVM implements an update function of the form $\mathbf{u}: \mathbf{S} \times \mathbf{S} \times \Lambda \rightarrow \mathbf{S}$. That is, the new state of a randomly chosen agent $i$ is given by

$$
y_{i}=\mathbf{u}\left(x_{i}, x_{j}, \lambda\right)=\left\{\begin{array}{cc}
x_{j} & : \lambda=\lambda_{V} \\
\bar{x}_{j} & : \lambda=\lambda_{C}
\end{array}\right\}
$$

where $\bar{x}_{j}$ denotes the opposite attribute of $x_{j}$. In each iteration, two agents $i, j$ are chosen along with a random variable $\lambda \in \Lambda=\left\{\lambda_{V}, \lambda_{C}\right\}$ that decides whether the voter $\left(\lambda_{V}\right)$ or the contrarian rule $\left(\lambda_{C}\right)$ is performed. The probability for that is $\omega(i, j, \lambda)$. Notice that the update rule is equal for all agents and independent from the agent choice. Therefore the probability that an agent pair $(i, j)$ is chosen to perform the contrarian rule can be written as 
$\omega(i, j) \operatorname{Pr}\left(\lambda=\lambda_{C}\right)=p \omega(i, j)$. Respectively, we have $(1-p) \omega(i, j)$ for the VM rule.

Let us briefly discuss the random mapping representation of the CVM for three agents and complete connections (i.e., $\omega(i, j)=1 / N(N-1), \forall i \neq j$ ). As shown in Table 5.2, this model variant implements 12 mappings $F_{z}$, because for any of the 6 possible agent choices $(i, j)$ there is an additional binary choice between the voter $\left(\lambda_{V}\right)$ and the contrarian $\left(\lambda_{C}\right)$ update mechanism. To index the set $\mathcal{F}_{Z}$ of mappings we now use a triple $(i, j, \lambda)$ where $i$ and $j$ correspond to the random agent choice and $\lambda=\left\{\lambda_{V}, \lambda_{C}\right\}$ to the random choice of one or the other rule. From Table 5.2 it is easy to see that the respective probability distribution $\omega(i, j, \lambda)$ is independent and identically distributed as it remains the same for any iteration at any time. That is to say, the micro-level process is a Markov chain.

\begin{tabular}{|c|c|c|c|c|c|c|c|c|c|}
\hline$z$ & $(i, j, \lambda)$ & a & $\stackrel{b}{b}$ & $\begin{array}{c}c \\
\square \\
\square\end{array}$ & ${ }^{\mathrm{d}}$ & $\begin{array}{c}\mathrm{e} \\
\square \square\end{array}$ & $\begin{array}{c}\mathrm{f} \\
\square \square\end{array}$ & $\begin{array}{c}\mathrm{g} \\
\square \square \square\end{array}$ & $\begin{array}{c}\mathrm{h} \\
\square \square \square\end{array}$ \\
\hline 1 & $\left(1,2, \lambda_{V}\right)$ & $\mathrm{a}$ & $\mathrm{b}$ & $\mathrm{g}$ & $\mathrm{a}$ & $\mathrm{h}$ & $\mathrm{b}$ & $\mathrm{g}$ & $\mathrm{h}$ \\
\hline 2 & $\left(1,2, \lambda_{C}\right)$ & d & $\mathrm{f}$ & $\mathrm{c}$ & d & e & $\mathrm{f}$ & $\mathrm{c}$ & e \\
\hline 3 & $\left(1,3, \lambda_{V}\right)$ & $\mathrm{a}$ & f & $\mathrm{c}$ & $\mathrm{a}$ & $\mathrm{h}$ & $\mathrm{f}$ & c & $\mathrm{h}$ \\
\hline 4 & $\left(1,3, \lambda_{C}\right)$ & d & b & g & d & e & $\mathrm{b}$ & g & e \\
\hline 5 & $\left(2,1, \lambda_{V}\right)$ & $\mathrm{a}$ & b & $\mathrm{a}$ & $\mathrm{g}$ & $\mathrm{b}$ & $\mathrm{h}$ & $\mathrm{g}$ & $\mathrm{h}$ \\
\hline 6 & $\left(2,1, \lambda_{C}\right)$ & $\mathrm{c}$ & e & $\mathrm{c}$ & d & e & $\mathrm{f}$ & d & $\mathrm{f}$ \\
\hline 7 & $\left(3,1, \lambda_{V}\right)$ & $\mathrm{a}$ & $\mathrm{a}$ & c & $\mathrm{f}$ & c & $\mathrm{f}$ & $\mathrm{h}$ & $\mathrm{h}$ \\
\hline 8 & $\left(3,1, \lambda_{C}\right)$ & $\mathrm{b}$ & $\mathrm{b}$ & e & d & $\mathrm{c}$ & d & g & g \\
\hline 9 & $\left(2,3, \lambda_{V}\right)$ & $\mathrm{a}$ & e & $\mathrm{a}$ & d & e & $\mathrm{h}$ & d & $\mathrm{h}$ \\
\hline 10 & $\left(2,3, \lambda_{C}\right)$ & c & $\mathrm{b}$ & c & $\mathrm{g}$ & $\mathrm{b}$ & $\mathrm{f}$ & $\mathrm{g}$ & $\mathrm{f}$ \\
\hline 11 & $\left(3,2, \lambda_{V}\right)$ & $\mathrm{a}$ & a & e & d & e & d & $\mathrm{h}$ & $\mathrm{h}$ \\
\hline 12 & $\left(3,2, \lambda_{C}\right)$ & $\mathrm{b}$ & $\mathrm{b}$ & $\mathrm{c}$ & $\mathrm{f}$ & $\mathrm{c}$ & $\mathrm{f}$ & g & $\mathrm{g}$ \\
\hline
\end{tabular}

Table 5.2: $\mathcal{F}_{Z}$ for the CVM with three agents.

Considering that $\lambda_{C}$ (for contrarian rule) is chosen with probability $p$ and $\lambda_{V}$ (VM rule) with $(1-p)$, and that this choice is independent of the agent choice, the micro-level transition probability $\hat{P}(\mathbf{x}, \mathbf{y})$ between two adjacent configurations $\mathbf{x} \stackrel{i}{\sim} \mathbf{y}$ is given by

$$
\hat{P}(\mathbf{x}, \mathbf{y})=(1-p) \sum_{j:\left(y_{i}=x_{j}\right)} \omega(i, j)+p \sum_{j:\left(y_{i}=\bar{x}_{j}\right)} \omega(i, j) .
$$

It is clear that, as for the original VM, the micro-level process for the CVM corresponds to a random walk on the hypercube. However, it is noteworthy that the CVM leads to a regular chain (as opposed to an absorbing random walk for the original VM). Namely, whenever $p>0$, there is a nonzero probability that the process leaves the consensus states $(\square \square \ldots \square)$ and

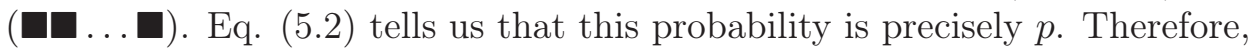


the system does not converge to a fixed configuration and the long-term behavior of the model can be characterized by its stationary distribution.

\subsection{Homogeneous Mixing and the Two-Community Graph}

This section analyses the behavior of the CVM for homogeneous mixing and the two-community graph. As seen in the previous chapter, for both interaction topologies there is a Markov projection which tremendously reduces the size of the chain such that the important entities of interest (e.g., stationary distribution) can be computed on the basis of the respective transition matrices.

\subsubsection{Homogeneous Mixing}

The case of homogeneous mixing is again particularly simple. Let us consider that the model is implemented on the complete graph without loops. That is, contrary to the treatment of the VM in Chapter 3, we do not allow that agents interact with themselves. In that case, the probability to choose a pair $(i, j)$ of agents becomes $\omega(i, j)=1 / N(N-1)$ whenever $i \neq j$ and $\omega(i, i)=0, \forall i$. As before, it is clear that the interaction structure is invariant with respect to all agent permutations (that is, $\omega(i, j)=\omega(\sigma i, \sigma j), \forall \sigma \in \mathcal{S}_{N}$ and all pairs $(i, j))$ and therefore all agent configurations with the same number $k$ of agents in $\square$ (and therefore $N-k$ in $\boldsymbol{\square}$ ) belong to the same class of macroscopic equivalence and can be mapped into the same macro atom $\left(X_{k}\right)$. See Sec. 3.3.1 and Prop. 4.3.1. In other words, for homogeneous mixing full aggregation over all agents does not destroy Markovianity, which is in complete analogy to the pure VM. Notice again that in hypercube terminology that level of observation corresponds to the Hamming weight of a configuration $h(\mathbf{x})=k$.

Consequently, since all agents interact with all the others with equal probability, the respective transition rates depend only on the numbers $k$ and $N-k$ of agents in the two states. Consider, for example, the probability $P\left(X_{k}, X_{k+1}\right)$ that a black agent flips its state. There are two situations in which this change can happen: first, if a pair of states $\left(x_{i}, x_{j}\right)=(\boldsymbol{\square}, \square)$ along with VM update is chosen, i.e., $\left(x_{i}, x_{j}, \lambda\right)=\left(\mathbf{\square}, \square, \lambda_{V}\right)$, second, if a pair $(\mathbf{\square}, \mathbf{\square})$ is chosen along with contrarian update, i.e., $\left(x_{i}, x_{j}, \lambda\right)=\left(\mathbf{\square}, \mathbf{\square}, \lambda_{C}\right)$. In a configuration with $k$ agents in $\square$, there are $(N-k) k$ possibilities for the first option and $(N-k)(N-k-1)$ possibilities for the latter. ${ }^{1}$

\footnotetext{
${ }^{1}$ Notice that in the case self-choice is allowed $(\omega(i, i)>0)$, the number of possibilities for $(\square, \square)$ modifies to $(N-k)^{2}$. We also have $\omega(i, j)=1 / N(N-1), \forall i \neq j$ compared to $1 / N^{2}$ in the model with self-choice. Even for systems of moderate size the dynamical effect of this slight difference in transition rates is neglectable.
} 
Alternatively, $P\left(X_{k}, X_{k+1}\right)$ can be obtained by evaluating the transition probability (5.2) from some $\mathbf{x} \in X_{k}$ to the set of $\mathbf{y} \in X_{k+1}$, denoted in the previous chapter as $\hat{p}_{\mathbf{x}, X_{k+1}}$ Then we obtain

$$
\begin{aligned}
P\left(X_{k}, X_{k+1}\right) & =\sum_{x_{i}=\boldsymbol{\square}}\left[(1-p) \sum_{j:\left(x_{j}=\square\right)} \omega(i, j)+p \sum_{j:\left(x_{j}=\square\right)} \omega(i, j)\right] \\
& =(N-k)[(1-p) k \omega+p(N-k) \omega] \\
& =(1-p) \frac{(N-k) k}{N(N-1)}+p \frac{(N-k)(N-k-1)}{N(N-1)} .
\end{aligned}
$$

Similarly, we obtain for $P\left(X_{k}, X_{k-1}\right)$

$$
P\left(X_{k}, X_{k-1}\right)=(1-p) \frac{(N-k) k}{N(N-1)}+p \frac{k(k-1)}{N(N-1)} .
$$

And finally,

$$
P\left(X_{k}, X_{k}\right)=\frac{k^{2}(2-4 p)+2 k N(2 p-1)+N(N-N p+p-1)}{N(N-1)}
$$

Fig. 5.1 aims at giving an intuition about the dynamical structure of the process by considering the relation between the probability for a transition one step to the right, $P\left(X_{k}, X_{k+1}\right)$, and a transition to the left, $P\left(X_{k}, X_{k-1}\right)$, as a function of $k$. This informs us about the more probable tendency for future evolution for every atom in the macro chain. Fig. 5.1 shows that $P\left(X_{k}, X_{k+1}\right)>P\left(X_{k}, X_{k-1}\right)$ for $k<N / 2$ and respectively $P\left(X_{k}, X_{k+1}\right)<$ $P\left(X_{k}, X_{k-1}\right)$ for $k>N / 2$ telling us that the contrarian rule (performed with probability $p$ ) introduces in every atom $X_{k}$ a small bias that drives the system towards the fifty-fifty configurations. This bias is given by

$$
P\left(X_{k}, X_{k+1}\right)-P\left(X_{k}, X_{k-1}\right)=p-\frac{2 k p}{N} .
$$
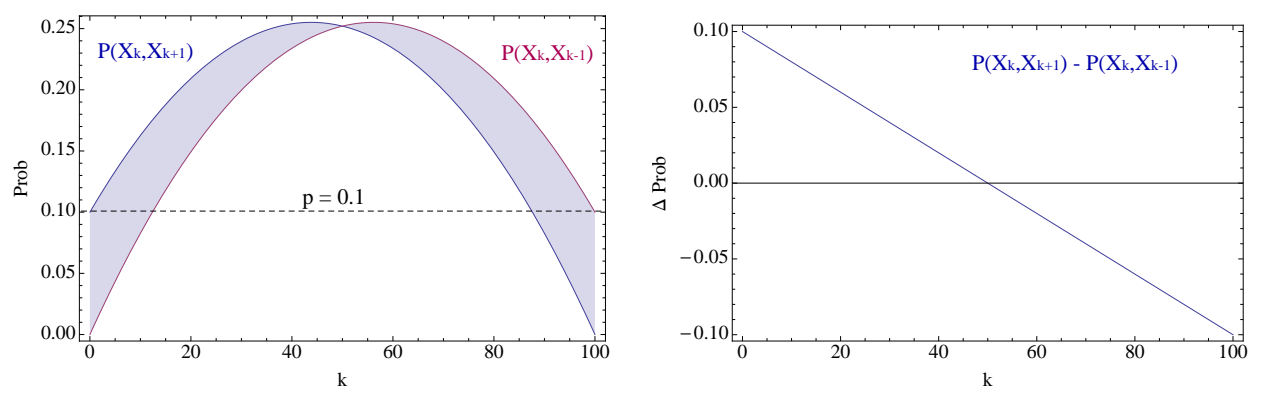

Figure 5.1: Transition probabilities and difference in transition probabilities as function of $k(N=100)$. 


\subsubsection{Stationary Dynamics for Homogeneous Mixing}

As already mentioned, contrary to the pure VM, the contrarian variant does no longer lead to an absorbing Markov chain, but results in a regular chain. In the case that the population reaches consensus $(k=0$ or $k=N)$ there is still a small probability, (namely $P\left(X_{0}, X_{1}\right)=P\left(X_{N}, X_{N-1}\right)=p$ ) with which the consensus configuration is left. In that case, a statistical understanding of the model behavior is provided by the limiting vector or stationary distribution it converges to. That is, by the distribution $\pi$ that remains unchanged under further application of $P$ :

$$
\pi P=\pi
$$

Notice that Eq. (5.7) tells us that the stationary distribution $\pi$ of a Markov chain $(\mathbf{X}, P)$ is proportional to the left eigenvector of $P$ associated to the maximal eigenvalue $\lambda_{\max }=1$. It is well-known (Kemeny and Snell, 1976, 69ff) that regular chains have a unique limiting vector and that the process converges to it for any initial distribution. Notice also, that the rate of convergence is usually related to the second largest eigenvalue of $P\left(\lambda_{2}<1\right)$ in the sense that the order of convergence is proportional to $\lambda_{2}^{t}$ (Kemeny and Snell, 1976; Behrends, 2000, among others).

For a system of 100 agents the stationary vector $\pi$ is shown in Fig. 5.2 for various contrarian rates $p$. The horizontal axis represents the macro states $X_{k}$ for $k=0,1, \ldots, N$ and the $\pi_{k}$ correspond to the probability with which the process is in atom $X_{k}$ provided it is run long enough and has reached stationarity. Notice that, for a large number of steps, the $\pi_{k}$ also represent the expected value for the fraction of time the process is in $X_{k}$ (Kemeny and Snell, 1976, Sec. 4.2). On the bottom of Fig. 5.2, three characteristic time series (three single simulation runs) are shown, one for large, one for intermediate and one for low $p$ values. This provides a better understanding of the meaning of the stationary vector in relation to the time evolution of the respective processes.

Two different regimes can be observed in Fig. 5.2 characterized by the green and the red curves respectively. A large contrarian rate $p$ (green curves) leads to a process which fluctuates around the states with approximately the same number of black and white agents - the fifty-fifty situation $(k=N / 2)$ being the most probable observation. The larger $p$, the lower the probability to deviate strongly from the fifty-fifty configurations. In fact, the process resembles a random process in which agent states are flipped at random.

A different behavior is observed if $p$ is small. This is represented by the red curves. For a small contrarian rate, the population is almost uniform (consensus) for long periods of time, but due to the random shocks introduced by the contrarian rule there are rare transitions between the two extremes. This is very similar to the VM at low (but non-zero) temperature, where random state switches or excitations take the role of mutations and 

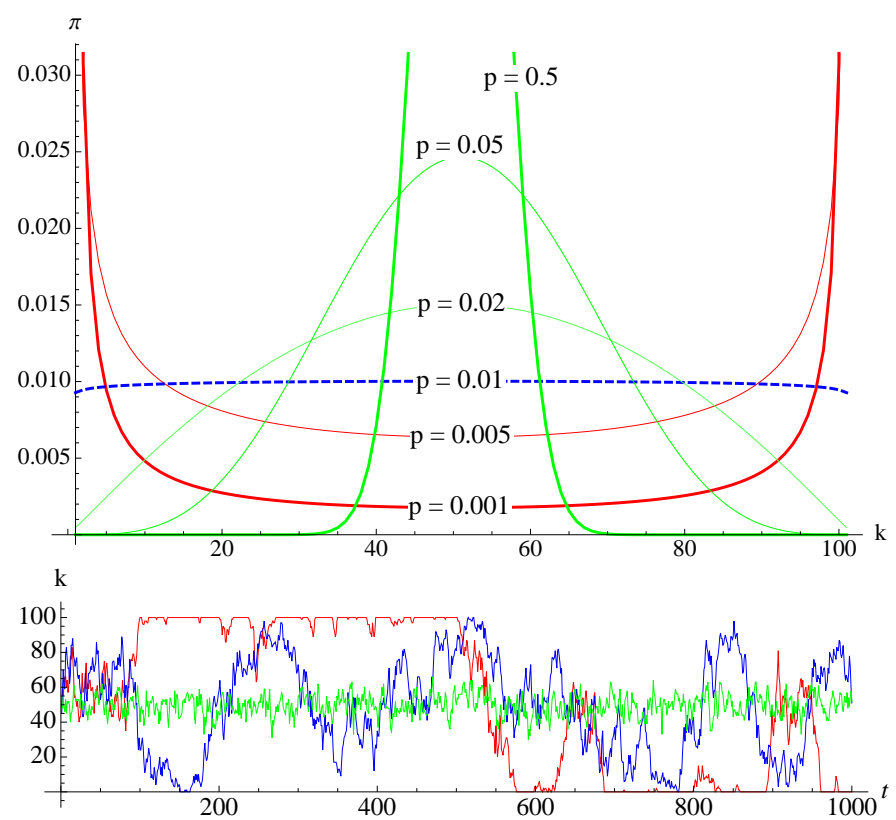

Figure 5.2: Stationary vector of the CVM with $N=100$ and homogeneous mixing for various $p$. There is a transition from the absorbing VM to the random fluctuations around the mean. On the bottom, the respective example time series are shown.

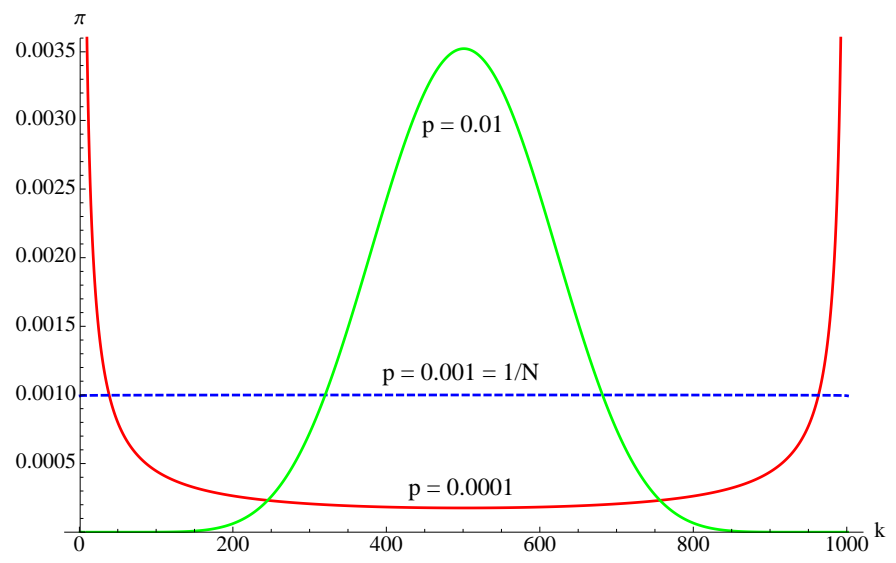

Figure 5.3: Stationary vector of the CVM with 1000 agents. Notice the (almost) uniform stationary distribution when $p=1 / N$. 
prevent the system from complete freezing to the zero-temperature ground state. In between these two regimes, there is a $p \approx 0.01$ for which the process wanders through the entire state space, in such a way that the stationary distribution is almost uniform.

Fig. 5.3 shows the situation for a system of 1000 agents. The same two regimes are observed for the larger system. However, the value $p$ at which the behavior changes from the switching between two consensus states to fluctuations around the fifty-fifty situation is decreased compared to the $N=$ 100 case. In the case of $N=1000$, an almost uniform stationary distribution is observed for $p \approx 0.001=1 / N$. To be more precise, it is, in fact, not difficult to show that for any system size $N$ the stationary distribution is uniform with $\pi_{k}=1 /(N+1), \forall k$ exactly for $p^{*}=1 /(N+1)$. All that is necessary in order to verify this is to show that $\pi P=\pi$ in this case. Hence, we have to show that

$$
\frac{1}{N+1}\left(P\left(X_{k-1}, X_{k}\right)+P\left(X_{k}, X_{k}\right)+P\left(X_{k+1}, X_{k}\right)\right)=\frac{1}{N+1}
$$

which is satisfied whenever

$$
P\left(X_{k-1}, X_{k}\right)+P\left(X_{k}, X_{k}\right)+P\left(X_{k+1}, X_{k}\right)=1 .
$$

Notice that Eq. (5.9) is equivalent to requiring that $P$ is a doubly stochastic matrix, and it is well-known that any doubly stochastic matrix has a uniform stationary vector. It is easy to show that for the CVM, Eqs. (5.8) and (5.9) are satisfied precisely for $p^{*}=1 /(N+1)$, but not for other contrarian rates.

When the contrarian rate $p$ crosses the critical value $p^{*}=1 /(N+1)$, the system undergoes a phase transition from majority-minority switching (ordered phase) to a balanced fifty-fifty situation in which no stable majorities form (disordered phase). A similar transition has been observed, for instance, in the contrarian model due to Galam (2004). The fact that $p^{*}=1 /(N+1)$ leads to $\pi_{k}=1 /(N+1), \forall k$ shows the existence of large fluctuations at the critical contrarian rate, because the only way to have a stationary uniform distribution is to have very large fluctuations at any value of the state space. For large $p$, the system behaves around the mean value (here 50) with only small deviations. For small $p$ closed to 0 , the system is rarely far from the two states of complete order (the consensus states) and in the limit of $p=0$ has no asymptotic fluctuations at all.

\subsubsection{Rate of Majority-Minority Switching}

One of the most interesting advantages of using Markov chains as a macro description of the model is that it facilitates the computation of a series of quantities that one might wish to look at and which are more difficult to assess with other techniques. For the CVM, for instance, we can look at the mean number of steps required to go from one consensus state to the 
opposite consensus state. As in the absorbing case, the key to this (and to several other) computations is a matrix called the fundamental matrix (Kemeny and Snell, 1976, p.75ff). For regular chains it is computed by

$$
\mathbf{F}=(\mathbf{I}-(P-W))^{-1}
$$

where $W$ is the limiting matrix with all rows equal to $\pi$ (note that, $\lim _{n \rightarrow \infty} P^{n}=$ $W)$. Following Kemeny and Snell (1976), the fundamental matrix can be used to compute another matrix $M$ which contains the mean number of steps between two states, say $i$ and $j$, for any pair of states:

$$
M=\left(\mathbf{I}-\mathbf{F}+\mathbf{E F}_{\text {diag }}\right) D
$$

where $\mathbf{E}$ is a matrix with all elements equal to one, $\mathbf{F}_{\text {diag }}$ the diagonal fundamental matrix with $\left(\mathbf{F}_{\text {diag }}\right)_{i i}=(\mathbf{F})_{i i} ;\left(\mathbf{F}_{\text {diag }}\right)_{i j}=0$, and $D$ the diagonal matrix with $(D)_{i i}=1 / \pi_{i}$. The mean time from one consensus state to the other is then given by the element $M(0, N)$ which is plotted in Fig. 5.4 for system sizes from $N=100$ to $N=500$.

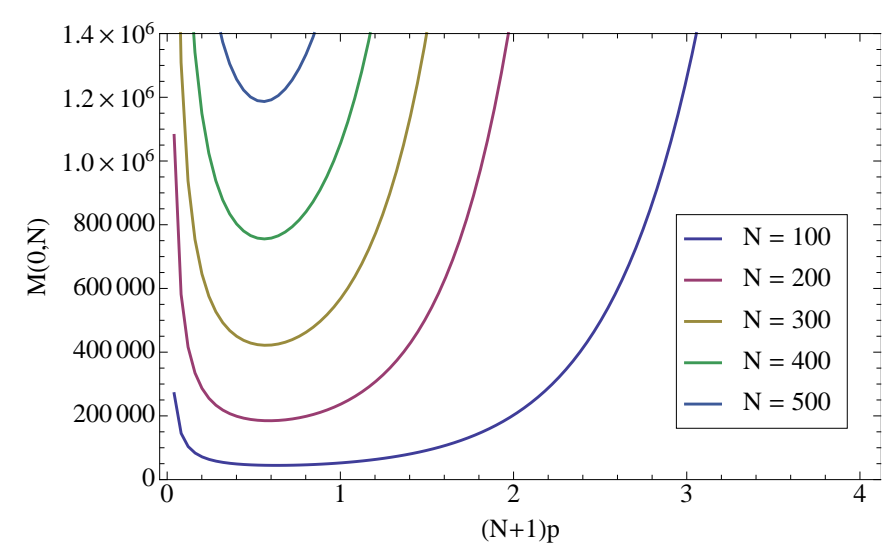

Figure 5.4: Mean number of steps required to go from one to the other consensus state as a function of the scaled contrarian rate $(N+1) p$.

Notice that in Fig. 5.4 the contrarian rate $p$ is scaled by the size of the macro chain $(N+1)$ in order to compare the different cases. This accounts for the above-mentioned fact that the $»$ critical « parameter value $p^{*}$ at which a uniform stationary distribution is found depends inversely on the number of agents as $p^{*}=1 /(N+1)$. Consequently, in Fig. 5.4, the uniform case is represented by $(N+1) p=1$. The switching behavior (from one consensus to the other and back) is found for values below that and the behavior approaches the random regime for values larger than one.

We observe in Fig. 5.4 that transitions between the two different consensus states are most frequent for a contrarian rate that is slightly below the 
$»$ critical $\ll$ contrarian rate $p^{*}$. There is a trade-off between the probability to indeed enter the state of complete consensus and the probability to go away from that and approach to the other extreme. For the $p$-values where $M(0, N)$ is minimal, both probabilities are relatively high. As contrarian rate decreases, the probability to reach consensus increases significantly, but a transition to the opposite consensus state is becoming rare. On the other hand, when $p$ increases slightly, transitions from $k \approx 0$ to $k \approx N$ and back are still rather likely, but in many case the process turns in direction before a complete ordering has been achieved. This is true also for $p \approx p^{*}$. As $p$ increases further, there is a strong decrease in probability to reach consensus altogether (see Fig. 5.2) and therefore the mean time between the two consensus states increases tremendously.

Finally, Fig. 5.5 shows the same analysis for transitions between states with a strong majority of $\square$-agents to an equally strong majority of $\square$-agents. The same qualitative behavior is observed in the sense that switching between strong majorities $\left(X_{0} \leftrightarrow X_{N}, X_{5} \leftrightarrow X_{95}, X_{10} \leftrightarrow X_{90}\right)$ becomes rather unlikely as the contrarian rate increases. On the other hand, transitions between moderate majorities of different sign ( $80 \%$ and respectively $67 \%$ ) occur rather frequently and the contrarian rate at which the mean time between them becomes minimal is larger.

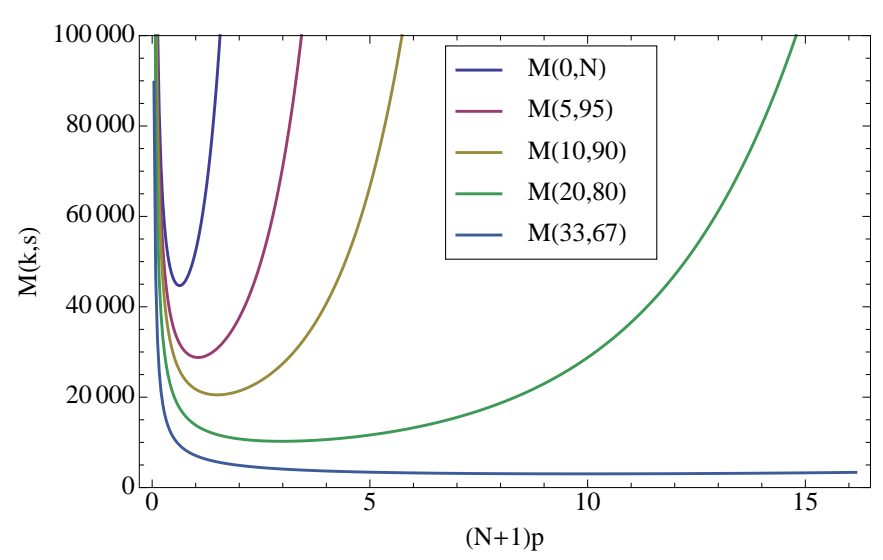

Figure 5.5: Mean number of steps required to go from $X_{k}$ to $X_{s}$ as a function of the scaled contrarian rate $(N+1) p$. Here $N=100$.

\subsubsection{Two-Community Model}

In this section, we consider the CVM on a two-community graph where the size of the two communities is given by $M=L=50$. The pure VM on two communities has been discussed in the previous chapter (Sec. 4.4) and for the CVM the same procedure can be used to obtain a Markov projection by strong lumpability; namely, Prop. 4.3.1. 
In particular, the description of the interaction topology in Sec. 4.4.1 and derivation of the respective interaction probabilities $\omega$ is in complete analogy to the previous chapter. That is,

$$
\begin{aligned}
\gamma & =\frac{r}{2 L M+((L-1) L+(M-1) M) r} \\
\alpha & =\frac{1}{2 L M+((L-1) L+(M-1) M) r},
\end{aligned}
$$

where $\gamma$ is the probability of intra-community interaction (strong ties) and $\alpha$ the probability of inter-community interaction (weak ties), and $r=\alpha / \gamma$ the ratio between the two.

As described in Sec. 4.4.2 for the pure VM, the interaction probabilities $\omega$ defined by Eqs. (5.12) give rise to a symmetry group $\operatorname{Aut}_{\omega}(N)=$ $(1 \ldots M)(M+1 \ldots N)$ and Prop. 4.3.1 tells us that Markovianity is preserved by a projection onto the $(M+1) \times(L+1)$ lattice. Each lattice point $\tilde{X}_{m, l}$ is associated to the attribute frequencies $m$ and $l$ within the two subcommunities. In other words, the model dynamics can be captured without loss of information by a »mesoscopic « formulation in terms of attribute frequencies $m$ and $l$ in the two communities. The state space of the projected model is visualized in Fig. 5.6.

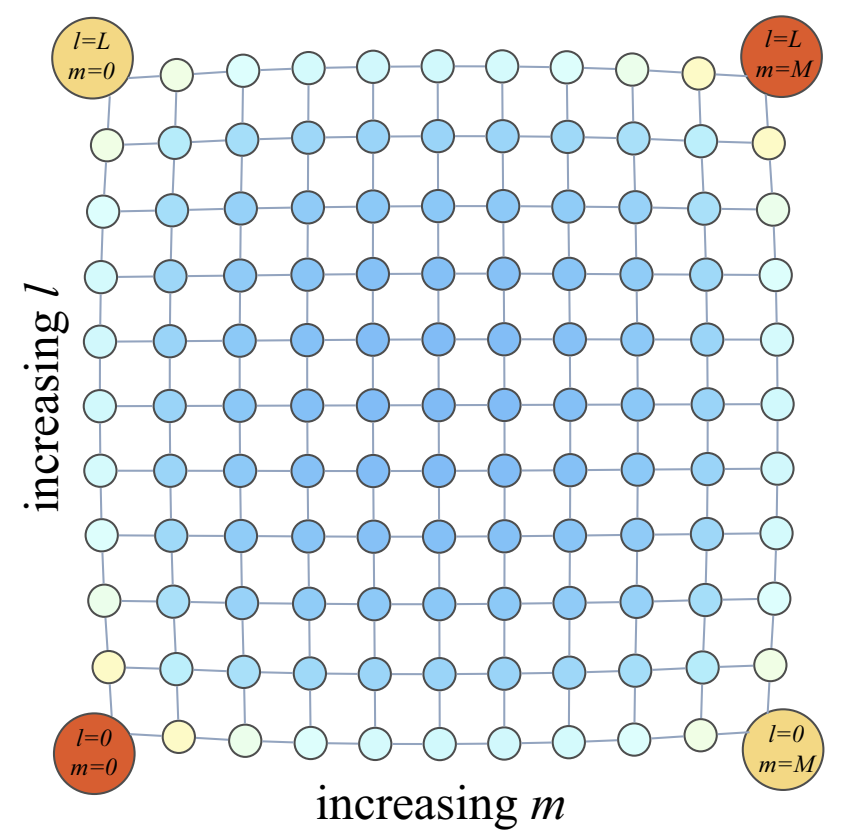

Figure 5.6: The structure of the CVM meso chain for $L=M=10$. The consensus states $\tilde{X}_{0,0}, \tilde{X}_{M, L}$ as well as the states of inter-community polarization $\tilde{X}_{0, L}, \tilde{X}_{M, 0}$ are highlighted. The stationary distribution is mapped into node colors from blue (low values) to red (high values). 
The colors shown in Fig. 5.6 represent the stationary distribution of the CVM with a relatively small contrarian probability $p$ and a very weak coupling between the two islands. The large atoms in the corners of the grid highlight the states that represent configurations of high order. On the one hand (red-shaded in Fig. 5.6) there are the consensus configuration with all agents in the same state: $\tilde{X}_{L, M}$ and $\tilde{X}_{0,0}$. On the other hand (yellowshaded), we have the states in which all agents of the same sup-group are aligned, but there is a disagreement across the sub-groups: $\tilde{X}_{0, M}$ and $\tilde{X}_{L, 0}$. As before, we refer to these states as inter-community polarization.

In what follows, we shall refer to the chain shown in Fig. 5.6 (obtained via strong lumpability) as meso chain and denote the state space as $\tilde{\mathbf{X}}=\left(\tilde{X}_{0,0}, \ldots, \tilde{X}_{m, l}, \ldots, \tilde{X}_{M, L}\right)$. The notion of $»$ meso $\ll$ in this context accounts for the fact that the process $(\tilde{\mathbf{X}}, \tilde{P})$ is indeed in between the micro and the macro level. Namely, it is a strong reduction compared to the microscopic chain $(\boldsymbol{\Sigma}, \hat{P})$, but the number of states is still considerably larger than the macro system $(\mathbf{X}, P)$ obtained by aggregation over the entire agent population $(h(\mathbf{x})=k)$. While the full aggregation compatible with homogeneous mixing has lead to a random walk on the line with $N+1=O(N)$ states, the two-community model leads to a random walk on a 2D lattice with $O\left(N^{2}\right)$ states. Noteworthy, the latter is a proper refinement of the former.

The transition probabilities of the meso chain are obtained on the basis of Eq. (5.2) by substitution of the respective interaction probabilities (5.12). That is, $\omega(i, j)=\gamma$ whenever two agent $i$ and $j$ are in the same community and $\omega(i, j)=\alpha$ whenever they are in different communities. For the CVM on two islands of size $M$ and $L$ the transition probabilities for the transitions leaving the atom $\tilde{X}_{m, l}$ are then given by

$$
\begin{aligned}
\tilde{P}\left(\tilde{X}_{m, l}, \tilde{X}_{m+1, l}\right)= & (1-p)[\gamma(m(M-m))+\alpha(M-m) l] \\
& +p[\gamma(M-m)(M-m-1)+\alpha(L-l)(M-m)] \\
\tilde{P}\left(\tilde{X}_{m, l}, \tilde{X}_{m-1, l}\right)= & (1-p)[\gamma(m(M-m))+\alpha m(L-l)] \\
& +p[\gamma m(m-1)+\alpha l m] \\
\tilde{P}\left(\tilde{X}_{m, l}, \tilde{X}_{m, l+1}\right)= & (1-p)[\gamma(L-l) l+\alpha(L-l) m] \\
& +p[\gamma(L-l)(L-l-1)+\alpha(L-l)(M-m)] \\
\tilde{P}\left(\tilde{X}_{m, l}, \tilde{X}_{m, l-1}\right)= & (1-p)[\gamma(L-l) l+\alpha(M-m) l] \\
& +p[\gamma l(l-1)+\alpha l m] .
\end{aligned}
$$

\subsubsection{Stationary Dynamics on the Two-Community Graph}

As described in Sec. 5.2.2, the stationary distribution $\pi$ of a Markov chain with transition matrix $P$ is the probability vector that satisfies $\pi P=\pi$. In other words, the computation of $\pi$ requires the computation of the left eigenvector of $P$. The Markov projection of the two-community model with $M=L=50$ results in a Markov chain of size $(M+1)(L+1)=2601$. For 

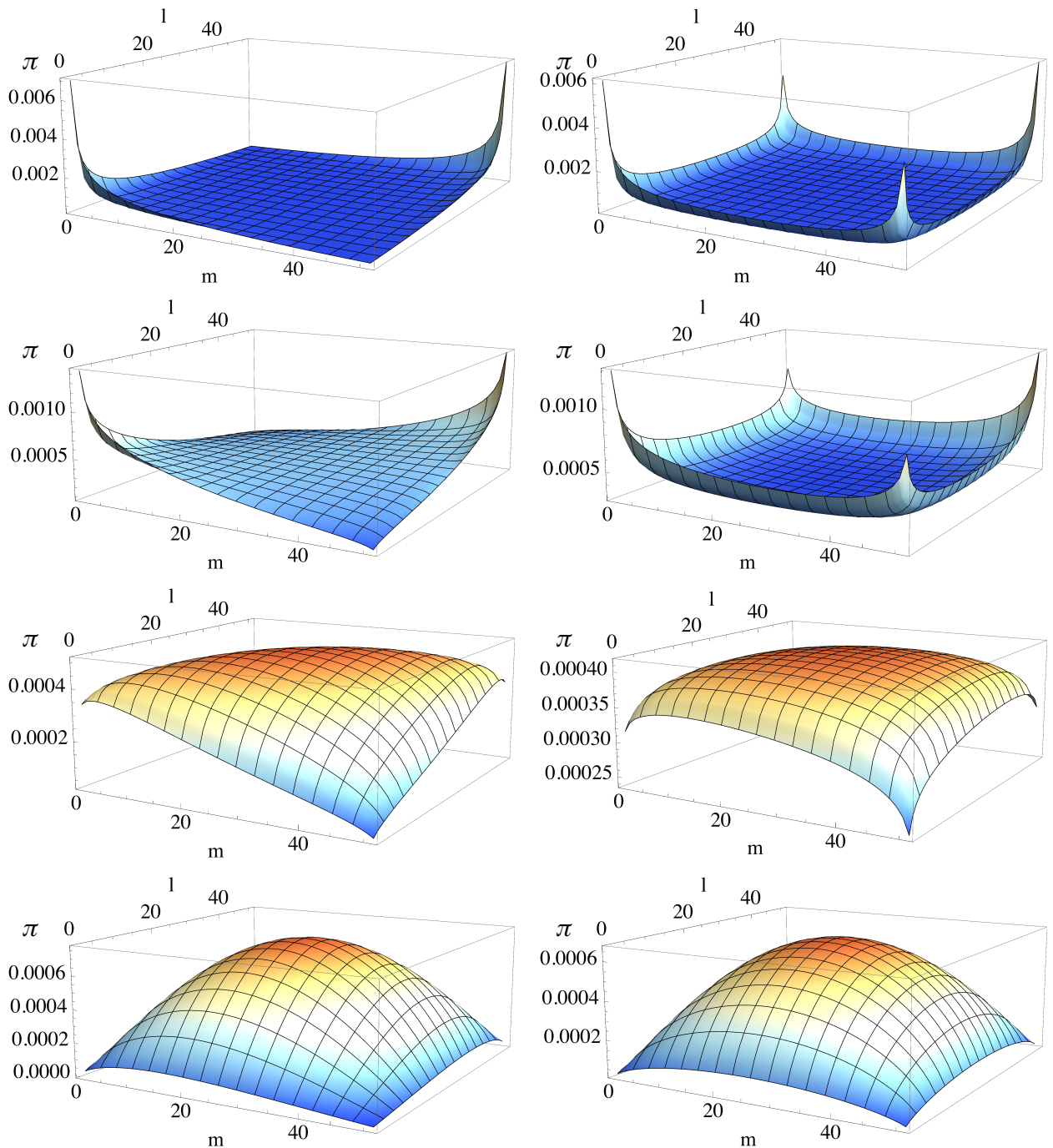

Figure 5.7: Stationary distribution for different $p$ and $r$ for a system of $M=L=50$. The column on the l.h.s. is for a moderate coupling $r=1 / 100$ and the four plots on the r.h.s. are for a weak coupling $r=1 / 1000$. From top to bottom the contrarian rates are $p=0.01,0.015,0.02,0.03$. The stationary probability for the consensus states $(m=l=0$ and $m=l=50)$ increases with decreasing $p$. The stationary probability for the states of partial order ( $m=M, l=0$ and $m=0, l=L$ ) increases as the coupling between the island $r$ decreases. This topological effect is undermined by an increasing contrarian rate $p$. 
a matrix of size $2601 \times 2601$ the (numerical) solution of the corresponding eigenvalue problem is still possible, but increasing the number of agents (that is, $M$ and $L$ ) will soon lead to matrix sizes for which the solution for eigenvalues and vectors is rather costly.

There are two parameters that decide about the dynamical behavior of the CVM on the two-community graph: (i.) the contrarian rate $p$, and (ii.) the coupling between the two islands captured by $r=\alpha / \gamma$. To obtain a complete picture of the model dynamics, the stationary distribution has been computed for various different values $p$ and $r$ which is shown in Fig. 5.7. From the top to the bottom, $p$ is increased from $p=0.01, p=0.015, p=0.02$ to $p=0.03$. The plots in the left-hand column show the result for a moderate coupling between the two island with $r=1 / 100$. A reduced coupling of $r=1 / 1000$ is shown in the plots in the right-hand column.

The comparison of the left- and the right-hand side of Fig. 5.7 shows that the stationary probability for states of inter-community polarization, as well as the states close to them, increases with a decreasing coupling between the communities. That is, the configurations with intra-community consensus, but disagreement across the communities become more and more probable. This is very obvious for the plots with a small contrarian rate $p=0.01$ and $p=0.015$ where the probability to observe the states $\tilde{X}_{0,50}$ or $\tilde{X}_{50,0}$ becomes very high when decreasing the coupling to $r=1 / 1000$. In fact, all configurations in which consensus is formed in at least one of the communities are rather likely (values along the border of the surface) whereas disordered configurations are rare. This is in direct analogy to the pure $\operatorname{VM}(p=0)$ and the corresponding quasi-stationary distribution (see Fig. 4.11). However, this significant difference between a moderate $(r=1 / 100)$ and a very weak $(r=1 / 1000)$ coupling diminishes as the contrarian rate becomes larger. This second trend observed in Fig. 5.7 is in agreement with what happens in the homogeneous mixing case as the contrarian rate $p$ increases: the probability to observe consensus configurations with all agents in equal state becomes more and more unlikely and it is more and more likely to observe disordered agent configurations all together. In fact, a further increase of the contrarian rate will lead to a behavior that is essentially random and insensitive to topological constraints since the consensus formation within the communities is frequently perturbed by random events.

In order to show that a decreasing inter-community coupling leads generally to an increased stationary probability of intra-community polarization (local alignment, global polarization), let us compare the previous cases to the homogeneous mixing situation $(r=1)$. This is shown in Fig. 5.8 for the (relatively small) contrarian rates $p=0.01$ and $p=0.03$. It becomes clear that for $\alpha=\gamma$ states of partial order $(m=M, l=0$ and $m=0, l=L)$ and states close to that become in effect exceptionally rare random events. The reason for this is clear. The number of micro configurations $\mathbf{x} \in \mathbf{\Sigma}$ mapped into the state $\tilde{X}_{m, l}$ is $\left(\begin{array}{c}M \\ m\end{array}\right)\left(\begin{array}{l}L \\ l\end{array}\right)$ which is a huge number for $m \approx M / 2, l \approx L / 2$ 

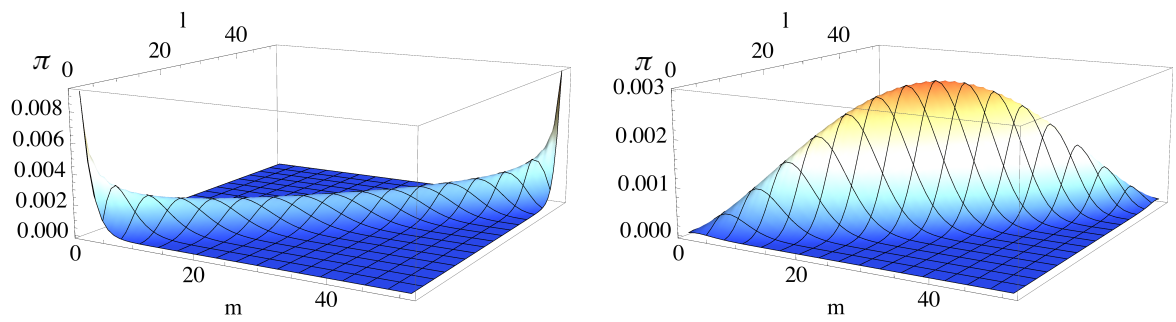

Figure 5.8: Stationary distribution for $p=0.01$ and $p=0.03$ for a system of $M=L=50$ and $\alpha=\gamma$ (homogeneous mixing). States of partial order $(m=M, l=0$ and $m=0, l=L)$ become a very rare random event.

but only 1 for $m=M, l=0$ and $m=0, l=L$. Because under homogeneous mixing there is no favoring of particular agent configurations with the same $k=m+l$ the stationary probability at meso scale is proportional to the cardinality of the set $\tilde{X}_{m, l}$.

\subsection{Network Dynamics from the Macro Perspective}

\subsubsection{Full Aggregation}

The most natural level of observation in binary state dynamics is to consider the temporal evolution of the attribute densities, or respectively, the number of agents in the two different states. While a mean-field description would typically formulate the macro dynamics a differential equation describing the evolution of attribute densities, the Markov chain approach operates with a discrete description (in time as well as in space) in which all possible levels of absolute attribute frequencies and transitions between them are taken into account. Regardless of the microscopic details such as more complex interaction networks or rules, a macro level description of that kind, which is always a tremendous reduction of original system, is desirable in order to obtain a better understanding of the model behavior. As a matter of fact, it is desirable for both numerical as well as analytical arguments.

One of the main contributions of the framework proposed here is that the link between the microscopic system and a certain macro level description is made explicit. Namely, a system property induces a partition on the space of all possible micro configurations and the macro-level process corresponds to the micro process projected onto that partition (see Chapter. 3, Sec. 3.2). For the CVM and many other binary single-step model the most relevant macro formulation corresponds to a projection of the micro-level process on the hypercube onto a macro-level process with the state space defined by the partition $\mathbf{X}=\left\{X_{0}, X_{1}, \ldots, X_{k}, \ldots, X_{N}\right\}$, where $0 \leq k \leq N$ corresponds to the number of $\square$-agents in the population. Each $X_{k}$ collects all micro configurations with $k$ with the same Hamming weight $h(\mathbf{x})=k$, that is, the 
same frequency level (see Sec. 3.3.1, Eqs. (3.12) and (3.13) in particular).

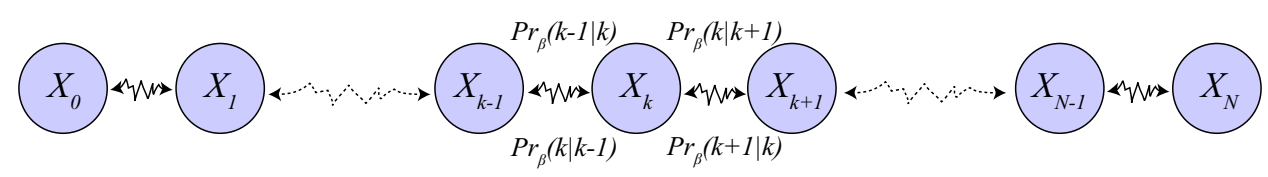

Figure 5.9: Full aggregation is obtained by the agglomeration of states with the same Hamming weight $h(\mathbf{x})=k$. The resulting macro process is, in general, a non-Markovian process on the line.

In this regard, one important observation in Chapter 3 has been that, for the VM, homogeneous mixing is a prerequisite for lumpability, and that microscopic heterogeneities (be it in the agents or in their connections) translate into dynamical irregularities that prevent lumpability with respect to $\mathbf{X}$. This means that full aggregation over the agent attributes $(k=h(\mathbf{x}))$ leads in general to a non-Markovian macro process. We illustrate this process in Fig. 5.9. Still, the process obtained by the projection from micro to macro is characterized by the fact that from an atom $X_{k}$ the only possible transitions are the loop to $X_{k}$, or a transition to neighboring atoms $X_{k-1}$ and $X_{k+1}$. This is of course due to the fact that the CVM implements single-step dynamics in which only one agent changes at a time. However, the micro level transition rates (5.2) depend essentially on the connectivity structure between the agents, and therefore, the transition probabilities at the macro level (denoted as $\operatorname{Pr}_{\beta}(l \mid k)$ in Fig. 5.9) are not uniquely defined (except for the case of homogeneous mixing) in the sense that for two configurations in the same macro state $\mathbf{x}, \mathbf{x}^{\prime} \in X_{k}$ the probability to go to another macro state (e.g., $X_{k+1}$ ) may be very different.

In this chapter, we use the CVM as a very simple model in which questions related to non-lumpable projections can be addressed. The scope is rather theoretical in the sense that we are interested in the kind of macro effects to which a non-trivial interaction structure at the micro level may lead. The questions we aim to address are of the following type: Why and in what sense does the behavior of the macro process deviate from Markovianity? Do we introduce memory or long-range correlations at the macro level by the very way we observe the process? Is the emergence of these effects just due to an aggregation which is insensitive to microscopic heterogeneities? And furthermore: How good does the mean field (homogeneous mixing) solution approximate network dynamics and for which networks does it provide acceptable approximations? Is there an alternative assignment of probabilities $\operatorname{Pr}_{\beta}(l \mid k)$ that leads to better results? Which properties can be captured? Finally, an interesting question concerns the reducibility of the micro chain by a weaker form of lumpability. At least to some of these question answers will be provided in the remainder of this chapter. 


\subsubsection{Network Influence on the Stationary Dynamics}

Let us first consider, in a numerical experiment, the effect of different interaction topologies $\omega$ on the stationary dynamics of the resulting macro process. For this purpose, we define the stationary macro measure $\pi$ as

$$
\pi_{k}=\sum_{\mathbf{x} \in X_{k}} \hat{\pi}_{\mathbf{x}}
$$

In other words, the elements $\pi_{k}$ of the stationary vector are determined by counting the frequency with which the model is in the respective set of micro states with $h(\mathbf{x})=k$. Notice that on the basis of a stationary micro chain, it is always possible to construct an approximate macro chain - an aggregation - the stationary vector of which satisfies Eq. (5.14) (see Kemeny and Snell, 1976, 140 and Buchholz, 1994, 61-63). This will be discussed below.

To compute the $\pi_{k}$, a series of simulations has been performed in which the CVM with $N=100$ is run on different paradigmatic agent networks. To capture the model in stationarity, the model is iterated for several thousands of steps first and the statistics of this »burn-in « phase are not considered in the computation of $\pi_{k}$. (In this exploratory analysis with 100 agents a $»$ burn-in « period of 20000 steps has been used.) The result is shown in Fig. 5.10 for the case of a small contrarian rate $p=0.005$.

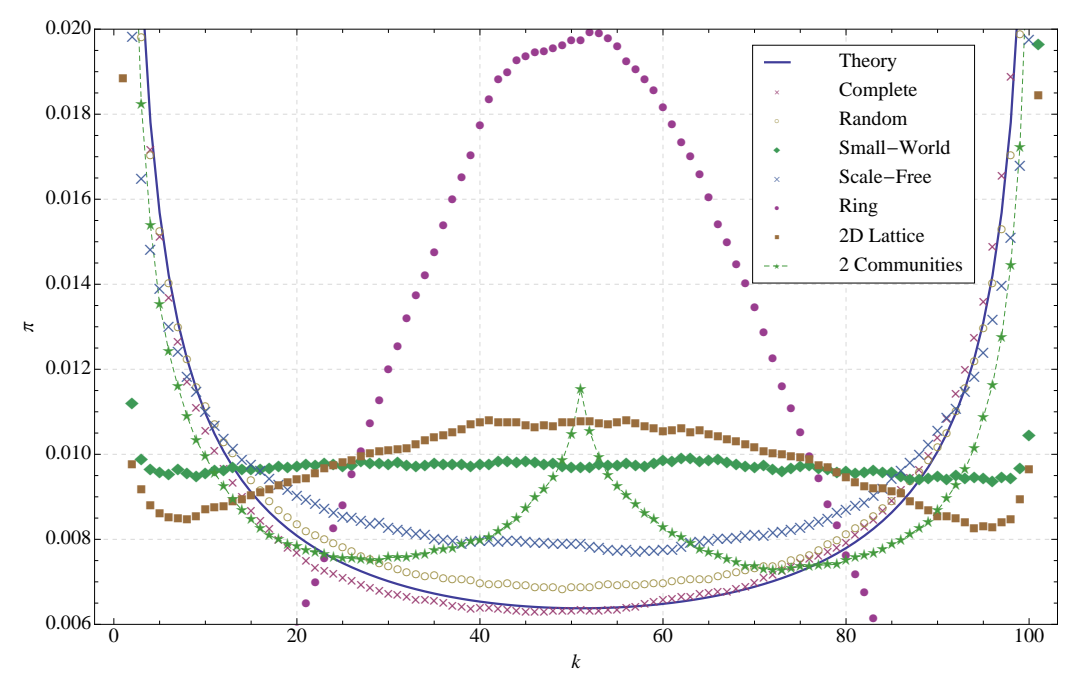

Figure 5.10: Stationary statistics for the CVM on different topologies. Due to effects of local ordering, the stationary behavior of the small-world network, the ring and the lattice as well as the two-community topology differs greatly from the well-mixed situations.

We observe in Fig. 5.10 that some interaction topologies give rise to strong deviations from the theoretical result derived for homogeneous mixing 
(solid, blue). In general, there is an increase in the probability to observe balanced configurations and the case of complete consensus tends to become less likely. However, the results obtained for the random graph are indeed very similar to the theoretical prediction and also the scale-free topology leads to stationary statistics that, in qualitative terms, correspond to the mean-field case. On the other hand, we observe a strong »modulation« of the stationary statistics by networks that tend to foster the emergence of »local alignment and global polarization«. By local alignment and the dynamics that lead to it, we refer to situations in which different clusters of agents approach independently a certain local consensus which is in general different from agent cluster to agent cluster. From the global perspective the entire population appears to be far from complete consensus and the probability to observe the respective intermediate macro states is increased. These effects are observed for the small-world network, the two-community graph as well as for the lattice, and it is strongest for the ring where the probability of complete consensus is practically zero.

\subsubsection{The Two-Community Case}

For the two-community graph with a peak around $k=N / 2$ the interpretation of the result is particularly straightforward. Local alignment, in this case, refers to inter-community polarization - the situation in which a different consensus has emerged in the two communities. If the size of the communities is $N / 2$, as in the example we study, the polarization configurations give rise to an macro observation $k=N / 2$ since one half of the population (organized in one community) agrees on $\square$ and the other half (that is, the other community) is in state

The two-community CVM is particularly interesting because we can compute the exact stationary vector by analyzing the respective meso chain $(\tilde{\mathbf{X}}, \tilde{P})$ obtained via strong lumpability. This has been done in Sec. 5.2.5. We first compute the stationary distribution of the meso chain assigning the respective limiting probability $\tilde{\pi}_{m, l}$ to each state $\tilde{X}_{m, l}$. In that case, Eq. 5.14 reads

$$
\pi_{k}=\sum_{m+l=k} \tilde{\pi}_{m, l}
$$

That is, $\pi_{k}$ associated to the macro state $X_{k}$ is obtained by summing up the respective $\tilde{\pi}_{m, l}$ with $m+l=k$. This is shown in Fig. 5.11 for different contrarian rates $p$ and different couplings between the two sub-graphs.

It becomes clear that the probability to observe a fifty-fifty situation $(k \approx N / 2)$ generally increases, the weaker the coupling between the communities. The analysis of the meso level stationary distribution shown in Fig. 5.7 makes clear that this is due to an increased probability for the configurations with intra-community consensus and inter-community polarization 

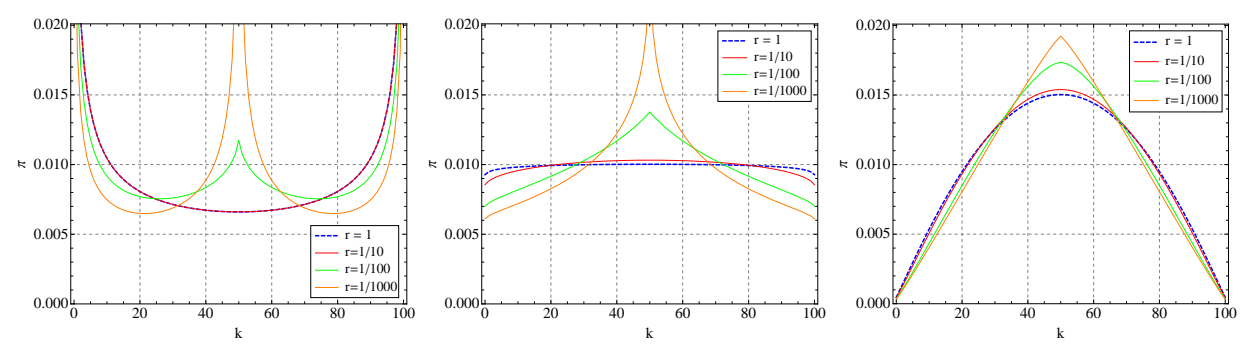

Figure 5.11: The stationary distribution from the macro perspective for different $r=\alpha / \gamma=1,1 / 10,1 / 100,1 / 1000$. From left to right $p=$ $0.005,0.01,0.02$.

$\left(\tilde{X}_{N / 2,0}, \tilde{X}_{0, N / 2}\right)$ which contribute to that probability. (Notice that the community sizes have a direct effect onto the macro level statistics and that in general the states with $k=L$ and $k=M$ will be observed more frequently when the coupling is weak.) In general, we can also observe that the influence of the different topological choices onto the macro behavior (captured here in terms of $\pi_{k}$ ) decreases with an increasing contrarian rate $p$. As explained in Sec. 5.2.5, the more contrarian behavior is allowed by the parameter setting, the more random becomes the entire process which undermines the effects of local alignment and, consequently, of interaction topology. This can be taken is a first indication that the mean-field solution (here represented by $r=1$ ) might approximate well the model behavior with a relatively high contrarian rate because the entire setting is characterized more and more by random state flips. It will be less accurate for a small contrarian rate where dynamics of local ordering become more and more characteristic.

\subsection{The Two-Community Model as an Analytical Framework}

\subsubsection{From Micro to Meso, and from Meso to Macro}

The previous section has shown that heterogeneous interaction structures can have a strong impact on the model behavior. From the lumpability point of view, but also from the point of view of observation, a macro process obtained by aggregation over the agent attributes neglects important information about the microscopic details. In other words, for heterogeneous networks in general, the micro-level process and the macro process do not commute, neither in an observational sense nor in the strict mathematical sense (Görnerup and Jacobi, 2008; Pfante et al., 2013). The only case where full aggregation provides us with a exact macro description is the homogeneous mixing case for which we have provided a Markov chain analysis in Sec. 5.2. 
We have also discussed in Sec. 5.2 the two-community graph for which a meso level chain with $O\left(N^{2}\right)$ states provides an loss-less coarse-graining. As the two-community coarse-graining $(\tilde{\mathbf{X}})$ is a proper refinement of the full aggregation $(\mathbf{X})$, and for it is still tractable, we may use this topology as a test case to address questions that concern the relation between the two coarse-grainings. Having available an aggregated mean-field-like description of the process $(\mathbf{X}, P)$ on the one hand, and a bigger Markov chain that describes exactly the model dynamics on an interaction topology with a small amount of inhomogeneity $(\tilde{\mathbf{X}}, \tilde{P})$ on the other, we can also analytically assess how the topology effects the macro dynamics and in which regard the network dynamics deviate from the mean-field behavior.

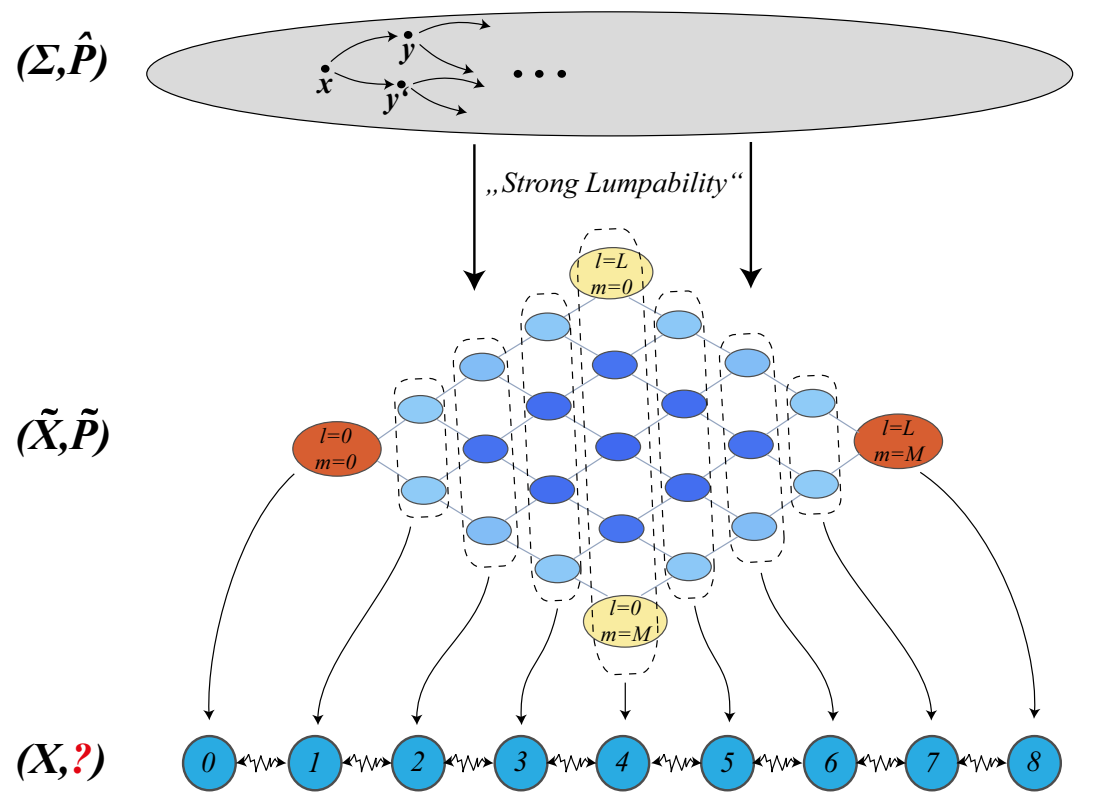

Figure 5.12: From micro to meso, and from meso to macro.

The general idea which we follow in this section is illustrated in Fig. 5.12. Consider the CVM on the two-community graph and the associated microlevel process $(\boldsymbol{\Sigma}, \hat{P})$. As shown in form of Prop. 4.3.1, the two-community micro chain $(\boldsymbol{\Sigma}, \hat{P})$ is (strongly) lumpable with respect to the partition $\tilde{\mathbf{X}}$. This gives rise to what we have called the meso-level process $(\tilde{\mathbf{X}}, \tilde{P})$ in Sec. 5.2.4. The meso-level Markov chain (meso chain) gives us a complete understanding of the (micro) behavior of the CVM on two coupled communities, because the coarse-graining via strong lumpability is compatible with the exact symmetries of the micro process and no information is lost. However, the process (the micro as well as the meso chain) is clearly not (strongly) lumpable with respect to the macro level of full aggregation (partition $\mathbf{X}$ ). Therefore, if we wish to observe the process at the global level, which is often the case in practice, we must live with the fact that the resulting macro pro- 
cess on $\mathbf{X}$ is no longer a Markov chain. As illustrated in Fig. 5.12, here we project onto the level of full aggregation despite the fact that Markovianity is lost, in order to understand (i.) the reasons for which lumpability is violated and (ii.) the dynamical effects that this introduces at the macro level. The fact that we have an explicit understanding of the meso chain facilitates an explicit analysis of the transition from micro to meso to macro.

\subsubsection{Why Lumpability Fails}

Let us first inspect the reasons for which the meso chain $(\tilde{\mathbf{X}}, \tilde{P})$ is not lumpable with respect to the macro partition $\mathbf{X}$. By the lumpability theorem (Kemeny and Snell, 1976, Thm. 6.3.2), it is clear that the non-lumpability of the meso chain with respect to $\mathbf{X}$ comes by the fact that the probabilities $\operatorname{Pr}\left(X_{k} \mid \tilde{X}_{m, l}\right)$ are not equal for all meso states $\tilde{X}_{m, l} \in X_{(m+l)}$ in the same macro set. As an example, let us consider the transition rates from the single $\tilde{X}_{m, l} \in X_{50}$ to the macro set $X_{51}$ in a system with $M=L=50$. One by one, the conjoint probability from $\tilde{X}_{0,50}, \tilde{X}_{1,49}, \tilde{X}_{2,48}, \ldots, \tilde{X}_{50,0}$ to the sets $\tilde{X}_{m, l} \in X_{51}$ is shown in Fig. 5.13 for various ratios $r$ and a small contrarian rate $p=0.01$.

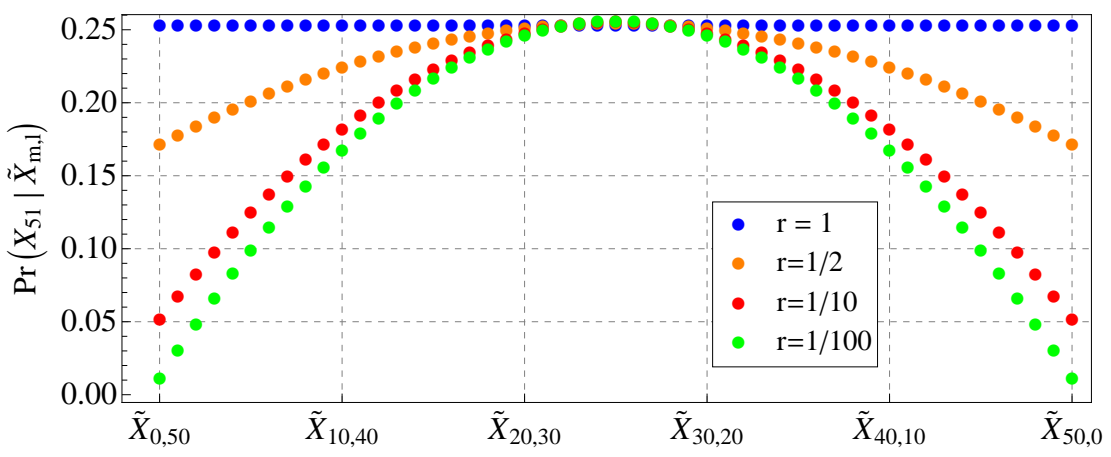

Figure 5.13: The island topology leads to inhomogeneous transition probabilities and is therefore not (strongly) lumpable. Here the example of a transition from $\tilde{X}_{m, l} \in X_{50}$ to $X_{51}$ in a system with $M=L=50$ is shown.

We first notice that the transition rates $\tilde{P}\left(\tilde{X}_{m, l}, X_{(m+l+1)}\right)$ are uniform when the coupling within is equal to the coupling across communities, that is for $\alpha=\gamma$ and $r=1$. Obviously, this is the case of homogeneous mixing and the uniformity of the $\tilde{P}\left(\tilde{X}_{m, l}, X_{(m+l+1)}\right)$ is precisely the lumpability condition spelled out in Thm. 6.3.2 of Kemeny and Snell (1976).

In general, the $\tilde{P}\left(\tilde{X}_{m, l}, X_{(m+l+1)}\right)$ are no longer equal for all $m$ and $l$ with $m+l=k$ when heterogeneity is introduced in form of a different coupling within and across communities, i.e., $\alpha \neq \gamma$. This explains the non-lumpability of the two-community model with respect to $\mathbf{X}$. As the weak ties across communities becomes weaker such that the ratio $r$ between 
strong and weak ties decreases, the transition rates become inhomogeneous, the main effect being a strong decrease of $\tilde{P}\left(\tilde{X}_{m, l}, X_{(m+l+1)}\right)$ for the atoms close to polarization $(m=M, l=0$ and $m=0, l=L)$. This decrease in transition probability, in turn, explains the increased stationary probability of the states $\tilde{X}_{50,0}$ and $\tilde{X}_{0,50}$, because once entered there is a relatively small probability to leave them so that the process is likely to »wait« in these states for quite some time.

Notice that there is only the small difference in transition rates between $r=1 / 10$ and $r=1 / 100$ (the difference to $r=1 / 1000$ is even smaller!). On the one hand, this is somewhat surprising, as from the dynamical point of view $r=1 / 10$ is much more related to the homogeneous mixing case $(r=1)$ than to the situation with $r=1 / 100$ (cf. Fig. 5.11). On the other hand, the probability to leave a polarized state $\left(\tilde{X}_{0,50}, \tilde{X}_{50,0}\right)$ decreases significantly with every decrease in $r$ and therefore the waiting times for these states grow tremendously. Notice, however, that in the limit of $r \rightarrow 0$, the probability of leaving a polarized state converges to $p$ (with $\left.\tilde{P}\left(\tilde{X}_{M, 0}, X_{(M+1)}\right)=\tilde{P}\left(\tilde{X}_{M, 0}, X_{(M-1)}\right)=p / 2\right)$. Therefore a strong difference between a weak (e.g., $r=1 / 100)$ and a very weak coupling $(r=1 / 1000)$ in form of an increased stationary probability of polarization can be expected only if also the contrarian rate $p$ is small. Likewise, as already observed in Sec. 5.2.5, a large contrarian rate can completely undermine effects of polarization altogether.

\subsubsection{Stationarity and Aggregation}

We shall now look at what happens to the macro level system as the micro or respectively meso process reaches stationarity. For this purpose we first look at the time evolution of the macroscopic transition rates. It is well-known that this measure (corresponding to the time dependent distribution over blocks of length two) converges in the case of an stationary macro process. We develop these ideas for a general micro chain $(\boldsymbol{\Sigma}, \hat{P})$ and show the twocommunity case (where we can indeed compute these entities) as an example.

Let $\hat{\beta}(0)$ denote the initial distribution over all micro configurations and $\hat{\beta}(t)$ be the respective distribution at time $t$. Notice that $\hat{\beta}(t)=\hat{\beta}(0) \hat{P}^{t}$. Let us further define the probability distribution at time $t$ restricted to the macro set $X_{k} \in \mathbf{X}$ as $\hat{\beta}^{k}(t)$. That is, the $\mathbf{x}$ th element $\hat{\beta}_{\mathbf{x}}^{k}(t)=0$ whenever $\mathbf{x} \notin X_{k}$ and proportional to $\hat{\beta}_{\mathbf{x}}(t)$ with

$$
\hat{\beta}_{\mathbf{x}}^{k}(t)=\frac{\hat{\beta}_{\mathbf{x}}(t)}{\sum_{\forall \mathbf{x}^{\prime} \in X_{k}} \hat{\beta}_{\mathbf{x}^{\prime}}(t)},
$$

for every $\mathbf{x} \in X_{k}$. Notice that by convention $\hat{\beta}_{\mathbf{x}}^{k}(t)=0$ whenever $\mathbf{x} \notin X_{k}$ and that $\hat{\beta}^{k}(t)$ is defined only if $\sum_{\mathbf{x}^{\prime} \in X_{k}} \hat{\beta}_{\mathbf{x}^{\prime}}(t)>0$, that is, if there is a positive 
probability that the process has reached at least one configuration $\mathbf{x}^{\prime} \in X_{k}$. The probability $\hat{\beta}_{\mathbf{x}}^{k}(t)$ shall be interpreted as the conditional probability that the process is in the configuration $\mathbf{x}$ at time $t$ provided that it is in the set $X_{k}$ at that time.

We now denote the expected transition probability from macro state $X_{k}$ to macro state $X_{s}$ as $\operatorname{Pr}_{\hat{\beta}(0)}^{t}\left(X_{s} \mid X_{k}\right)$. With $\hat{\beta}^{k}(t)$ defined as above, it is given by

$$
\operatorname{Pr}_{\hat{\beta}(0)}^{t}\left(X_{s} \mid X_{k}\right)=\sum_{\mathbf{x} \in X_{k}}\left[\hat{\beta}_{\mathbf{x}}^{k}(t) \sum_{\mathbf{y} \in X_{s}} \hat{P}(\mathbf{x}, \mathbf{y})\right]
$$

For the interpretation of Eq. (5.17) consider that $\hat{\beta}_{\mathbf{x}}^{k}(t)$ is the probability (restricted to $X_{k}$ ) that the process is in $\mathbf{x} \in X_{k}$ at time $t$ and $\sum_{\mathbf{y} \in X_{s}} \hat{P}(\mathbf{x}, \mathbf{y})=$ $\hat{P}\left(\mathbf{x}, X_{s}\right)$ is the probability for a transition from $\mathbf{x}$ to some $\mathbf{y} \in X_{s}$. A transition from the set $X_{k}$ to $X_{s}$ is then the conjoint transition probability considering all $\mathbf{x} \in X_{k}$ along with their conditional probability $\hat{\beta}_{\mathbf{x}}^{k}(t)$ (first sum). Notice again that (5.17) corresponds to the probability of observing a sequence of two measurements $(h(\mathbf{x}), h(\mathbf{y}))=(k, s)$ at a certain time $t$ when looking at the micro system through the eye of absolute attribute frequencies.

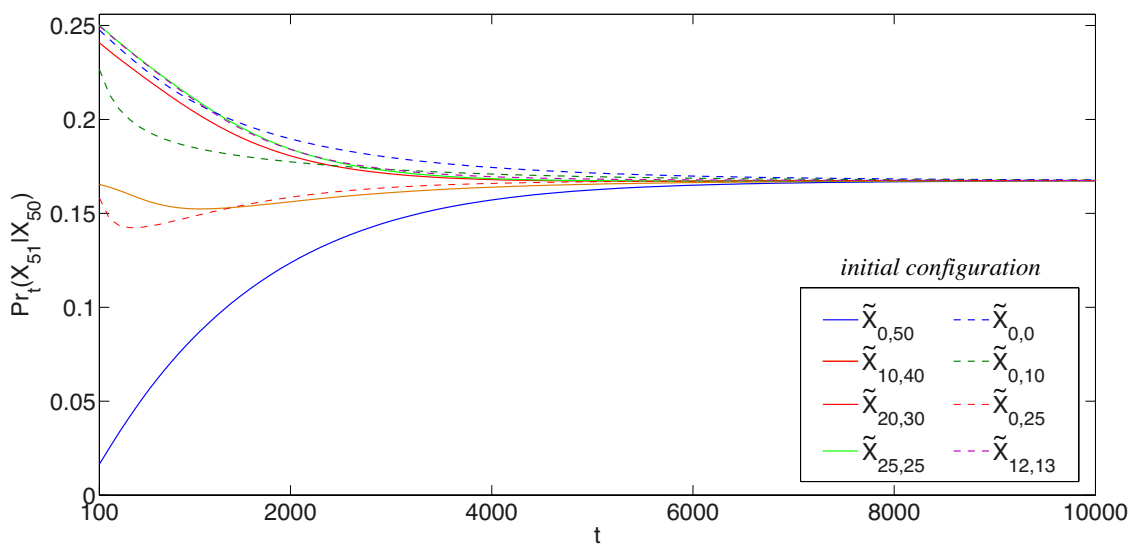

Figure 5.14: Time evolution of transition rates $\operatorname{Pr}_{\tilde{\beta}(0)}^{t}\left(X_{51} \mid X_{50}\right)$ from $X_{50}$ to $X_{51}$ in the two-community model for some of the meso states considered in Fig. 5.13 and different initial conditions.

Now, notice that the only time dependent term in Eq. (5.17) is the conditional distribution $\hat{\beta}_{\mathbf{x}}^{k}(t)$ which is obtain by $(5.16)$ from $\hat{\beta}(t)$, and $\hat{\beta}(t)=$ $\hat{\beta}(0) \hat{P}^{t}$. Considering that $(\boldsymbol{\Sigma}, \hat{P})$ is regular, it is clear that the process reaches its stationary state $\left(\lim _{t \rightarrow \infty} \hat{\beta}(t)=\hat{\pi}\right)$ independent of the initial $\hat{\beta}(0)$. Therefore, 
the $\operatorname{Pr}_{\hat{\beta}(0)}^{t}\left(X_{s} \mid X_{k}\right)$ converge to

$$
\operatorname{Pr}_{\hat{\pi}}\left(X_{s} \mid X_{k}\right)=\sum_{\mathbf{x} \in X_{k}}\left[\hat{\pi}_{\mathbf{x}}^{k} \sum_{\mathbf{y} \in X_{s}} \hat{P}(\mathbf{x}, \mathbf{y})\right]
$$

as the micro process reaches stationarity. See Fig. 5.14 for the two community model. Consequently (Kemeny and Snell, 1976; Buchholz, 1994), Eq. (5.18) can be interpreted as a macroscopic transition matrix with $P\left(X_{k}, X_{s}\right)=\operatorname{Pr}_{\hat{\pi}}\left(X_{s} \mid X_{k}\right)$, and the stationary vector of that matrix will be correct in the sense of (Eq. 5.14).

The possibility of deriving such a macro description has been commented on by Kemeny and Snell (1976), page 140, and it is discussed with some detail by Buchholz (1994), pages $61-63$, where it is referred to as an ideal aggregate. The most important thing to notice (Kemeny and Snell, 1976, 140) is that $P^{2}$ does not correctly describe the two-step transition probabilities that would be measured on the micro system. That is, the system evolution described solely at the aggregated macro level is different from the macro evolution that would be observed by running the microscopic process and performing an aggregation after each micro step. In other words (cf. Pfante et al., 2013), as some information about the dynamical behavior of the microscopic system is omitted by the aggregation, it violates a commutativity condition and in our case this violation is equal to non-lumpability. In fact, one can basically look at an ideal aggregate obtained by (5.18) as a Markov model that approximates a certain stationary process (in our case the macro process obtained by measurements from the micro chain) on the basis of the empirical distribution of cylinders of length two. It is in fact not clear whether the process is informative about certain properties of the real macro process beyond the stationary measure (see Sec. 2.4.3). Finally, even if the chain defined by (5.18) would be informative about certain transient properties of the real macro process, it still suffers from the fact that the construction of it requires knowledge of the stationary distribution of the micro chain $\hat{\pi}$ which is usually unknown.

Notice that Eqs. (5.17) and (5.18) do not involve any particular assumption on the nature of the partition meaning that an ideal aggregate can be constructed by them for any partition of $\boldsymbol{\Sigma}$. Buchholz (1994), Thm. 1, has shown that if the original transition matrix ( $\hat{P}$ in our case) is irreducible than the transition matrix of the ideal aggregate $P\left(X_{k}, X_{s}\right)=\operatorname{Pr}_{\hat{\pi}}\left(X_{s} \mid X_{k}\right)$ will also be irreducible and therefore possess a unique stationary distribution.

\subsubsection{Why Weak Lumpability Fails}

Weak lumpability (see Sec. 2.4.2) refers to the fact that a Markov chain might be lumpable only for particular starting vectors (Burke and Rosenblatt, 1958; Kemeny and Snell, 1976; Ledoux et al., 1994). The question 
whether or not an ideal aggregate (and hence the micro chain) is weakly lumpable arises naturally from our construction of an ideal aggregate, (5.17) and (5.18), mainly by two considerations: first, it is well-known that if a chain is weakly lumpable with respect to some distribution, it must be lumpable with respect to the stationary distribution; and second, the transition probabilities of the lumped process would be given by Eq. (5.18) (Kemeny and Snell, 1976, Thm. 6.4.3). Therefore questions of weak lumpability of the micro process with respect to full aggregation $\mathbf{X}$ can be answered by checking if the ideal aggregate is lumpable.

For the two-community model it is in fact easy to show that the CVM process is not weakly lumpable by the construction of a counter example which shows that the conditions of Thm. 6.4.1 in Kemeny and Snell (1976) are violated. The argument is two-fold. First, starting from $\hat{\pi}$ the process generally reaches different assignments of probabilities over the micro states in the different macro sets (different $\hat{\beta}^{s}$ ), because, at least for the two-community model,

$$
\left(\pi^{k} P\right)^{s} \neq \pi^{s}
$$

The superscripts $k$ and $s$ denotes, as before, restriction to $X_{k}$ and $X_{s}$ respectively. Let us denote the left-hand side of (5.19) as $\hat{\pi}^{\prime s}=\left(\pi^{k} P\right)^{s}$. Notice that, in fact, for weak lumpability it would be sufficient to show that $\hat{\pi}^{\prime s}=\pi^{s}$ is satisfied for any $k$ and $s$ (cf. Kemeny and Snell (1976), p.136). However, even if the situation is as in (5.19), weak lumpability could still be the case if the two distribution $\hat{\pi}^{\prime s}$ and $\pi^{s}$ lead to the same transition probabilities to all other macro sets $X_{l}$

$$
\operatorname{Pr}_{\hat{\pi}}\left(X_{l} \mid X_{s}\right)=\operatorname{Pr}_{\hat{\pi}^{\prime}}\left(X_{l} \mid X_{s}\right)
$$

In other words, weak lumpability (according to Kemeny and Snell, 1976, Thm. 6.4.1) is violated if the probability of a transition from $X_{s}$ to another macro state $X_{l}$ is different for $\hat{\pi}^{s}$ and $\hat{\pi}^{\prime s}$. This is in general the case for the two-community model, as will be shown in the sequel.

As an example, let us consider a small system with $M=L=2$. That is, the two communities each consist of only two agents. Let us say the process is in equilibrium with distribution $\hat{\pi}$ at time $t$. Now we consider the macro probability $X_{2} \rightarrow X_{1}, \operatorname{Pr}_{\hat{\pi}}\left(X_{1} \mid X_{2}\right)$, which is given by:

$$
\operatorname{Pr}_{\hat{\pi}}\left(X_{1} \mid X_{2}\right)=\frac{(1+r-p)(2 r(-1+p)-p)(1+p)}{2\left(-1+2 r^{2}(-2+p)+2 p-3 p^{2}+r\left(-1-7 p+6 p^{2}\right)\right)}
$$

for arbitrary $r$ and $p$. Let us further assume that the process performs a loop in the first step $(t \rightarrow t+1)$ and transits to $X_{1}$ only after that (in $t+1 \rightarrow t+2$ ). That is, $X_{2} \rightarrow X_{2} \rightarrow X_{1}$. For weak lumpability with starting vector $\hat{\pi}$ the probability of $X_{2} \rightarrow X_{1}$ must be the same independent of how many and which previous steps are taken. However, for the second case we 
have $\hat{\pi}^{\prime 2}=\left(\pi^{2} P\right)^{2} \neq \pi^{2}$ and then

$\operatorname{Pr}_{\hat{\pi}^{\prime}}\left(X_{1} \mid X_{2}\right)=\frac{(1+r-p)(2 r(-1+p)-p)\left(1-2 p-4 r(-2+p) p+3 p^{2}+r^{2}\left(2+4 p^{2}\right)\right)}{2(1+2 r)^{2}\left(1-3 p+3 p^{2}+p^{3}+2 r^{2}\left(1-p+p^{2}\right)-r\left(1-8 p+5 p^{2}+2 p^{3}\right)\right)}$,

which is obviously not equal to (5.21). This shows that the two-community model is not weakly lumpable with respect to $\mathbf{X}$.
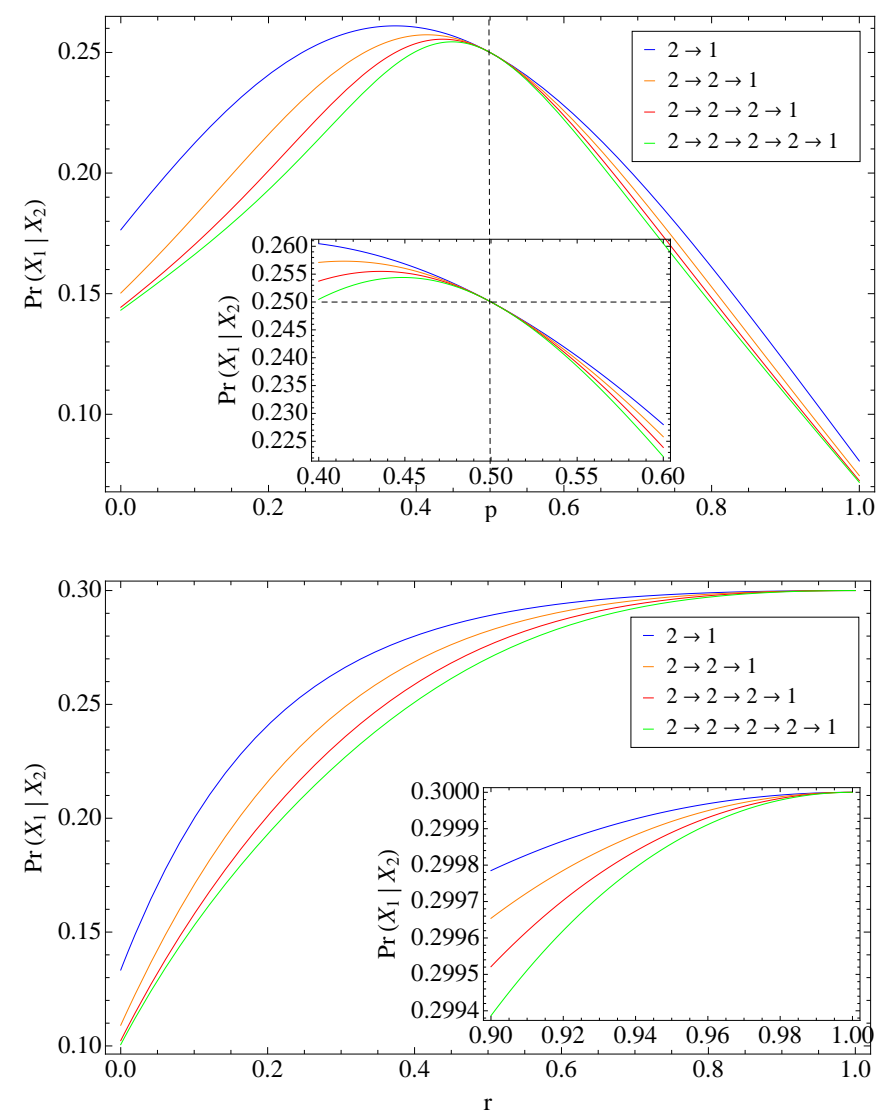

Figure 5.15: Transition probabilities $\operatorname{Pr}_{\hat{\beta}}\left(X_{1} \mid X_{2}\right)$ for $\hat{\beta}=\hat{\pi}, \hat{\pi}^{\prime}, \hat{\pi}^{\prime \prime}, \hat{\pi}^{\prime \prime \prime}$ for the small example $M=L=2$ are not equal as would be required for weak lumpability. Top: $\operatorname{Pr}_{\hat{\beta}}\left(X_{1} \mid X_{2}\right)$ is shown as a function of $p$ for $r=1 / 5$. The curves converge to the same value at $p=1 / 2$. Bottom: $\operatorname{Pr}_{\hat{\beta}}\left(X_{1} \mid X_{2}\right)$ is shown as a function of $r$ for $p=1 / 5$. Equal probabilities are observed for the strongly lumpable case $r=1$.

In Fig. 5.15 we show the probabilities $\operatorname{Pr}_{\hat{\beta}}\left(X_{1} \mid X_{2}\right)$ for the cases from $X_{2} \rightarrow X_{1}$ to $X_{2} \rightarrow X_{2} \rightarrow X_{2} \rightarrow X_{2} \rightarrow X_{1}$ as a function of $p$ (top) and $r$ (bottom). As we would expect (see figure on the bottom and the inset) the curves approach the same value as $r \rightarrow 1$. This is the strongly lumpable case of homogeneous mixing. Interestingly, we observe in the upper image 
of Fig. 5.15 that the probabilities are actually equal for $p=1 / 2$, namely $\operatorname{Pr}_{\hat{\beta}}\left(X_{1} \mid X_{2}\right)=1 / 4$ in that case. See the respective inset in the upper figure. This indicates lumpability of the process for $p=1 / 2$ and, in fact, it is possible to show that the two-community model is strongly lumpable whenever $p=$ $1 / 2$. The reason is that for $p=1 / 2$, the meso-level transition matrix $\tilde{P}$ is independent of the topological parameter $r$. Even if the case $p=1 / 2$ is not that interesting from the point of view of the dynamical behavior of the CVM, it would be interesting to check whether a similar effect also occurs for other networks.

\subsubsection{Measuring (Non)-Markovianity}

Having shown that the macro process associated to the CVM on two coupled communities is non-Markovian, the next logical step is to quantify in some way the deviations from Markovianity. The framework of information theory - relative entropy and mutual information in particular - has been shown to be quite useful for this purpose (Chazottes et al., 1998; Vilela Mendes et al., 2002; Görnerup and Jacobi, 2008; Ball et al., 2010; James et al., 2011; Pfante et al., 2013, among others).

Let us, to simplify the writing, denote as $\left[\ldots, k_{t-2}, k_{t-1}, k_{t}, k_{t+1}, k_{t+2, \ldots}\right]$ a sequence of macro states $\ldots \rightarrow X_{k_{t-2}} \rightarrow X_{k_{t-1}} \rightarrow X_{k_{t}} \rightarrow X_{k_{t+1}} \rightarrow X_{k_{t+2}} \rightarrow$ ... Likewise, let us denote as $\left[k_{t-m}, \ldots, k_{t}\right]$ a finite sequence of $m$ macro states and refer to this as block or cylinder of length $m$. Then, the block entropy associated to cylinders of length $m$ is defined by

$$
H_{m}=\sum_{\left[k_{t-m}, \ldots, k_{t}\right] \in \mathfrak{G}_{m}} \mu\left(\left[k_{t-m}, \ldots, k_{t}\right]\right) \log \mu\left(\left[k_{t-m}, \ldots, k_{t}\right]\right)
$$

where $\mu\left(\left[k_{t-m}, \ldots, k_{t}\right]\right)$ denotes the probability to observe the respective cylinder $\left[k_{t-m}, \ldots, k_{t}\right]$. Notice that for $m>1$ there exist in general $\gg$ forbidden « sequences with $\mu\left(\left[k_{t-m}, \ldots, k_{t}\right]\right)=0$, a fact that is usually formalized in terms of a grammar $\mathfrak{G}_{m} \subseteq \mathbf{X}^{m}$ by defining $\mathfrak{G}_{m}:=\left\{\left[k_{t-m}, \ldots, k_{t}\right]\right.$ : $\left.\mu\left(\left[k_{t-m}, \ldots, k_{t}\right]\right)>0\right\}$. In our case of single-step dynamics, all sequences containing subsequent elements with $\left|k_{t}-k_{t-1}\right|>1$ are $»$ forbidden « because only $X_{k}, X_{k-1}$ and $X_{k+1}$ can be reached from $X_{k}$ in one step.

It is well-known (Chazottes et al., 1998; Vilela Mendes et al., 2002; James et al., 2011) that the slope of the block entropy $\Delta H_{m}=H_{m}-H_{m-1}$ converges to a fixed value called entropy rate (usually denoted as $h(\mu)$ ) and that this fact can be used to estimate the memory range of the process. Namely, following Chazottes et al. (1998); Vilela Mendes et al. (2002), the range of the process is given, at least in an approximative sense, by the $m$ at which $\Delta H_{m}$ reaches a constant value, that is, $\Delta H_{m}-\Delta H_{m+1} \approx 0$. It is clear then that for a Markovian process this point must be reached at $m=2$ such that

$$
\Delta H_{2}-\Delta H_{3}=0
$$


and more generally

$$
\Delta H_{2}-\Delta H_{m}=0
$$

Notice that Eq. (5.25) is precisely the $»$ Markov property measure« proposed in Görnerup and Jacobi (2008), pp.6-8, to identify projections of a process onto a smaller state space (a partition of the original process) which lead to Markovian dynamics. Noteworthy, the starting point of Görnerup and Jacobi (2008) is the expected mutual information $\langle I\rangle$ between pasts and the future state. Namely, how much information about the next symbol $\left(\left[k_{t+1}\right]\right)$ is on average over all symbols contained in the sequence of symbols $\left(\left[\ldots, k_{t-2}, k_{t-1}\right]\right)$ before the current symbol $\left(\left[k_{t}\right]\right)$. They show that the expected past future mutual information can be expressed in terms of the slopes of the block entropy as

$$
\langle I\rangle=\Delta H_{2}-\Delta H_{\infty}
$$

and likewise

$$
\left\langle I_{n}\right\rangle=\Delta H_{2}-\Delta H_{2+n}
$$

if finite histories of length $n\left(\left[k_{t-n} \ldots, k_{t-2}, k_{t-1}\right]\right)$ are considered. ${ }^{2}$ Notice that in their notation $n$ accounts for the ranges beyond the Markov range of two $(m=n+2$ in Eq. 5.25). We will follow this notation here and compute $\left\langle I_{1}\right\rangle=\Delta H_{2}-\Delta H_{3}$ and $\left\langle I_{2}\right\rangle=\Delta H_{2}-\Delta H_{4}$, the latter being used by Görnerup and Jacobi (2008).

The advantage of the two-community CVM as a framework to link between a micro and a macro level of description via an intermediate meso level description is that we are able to compute the Markovianity measures $\left\langle I_{1}\right\rangle$ and $\left\langle I_{2}\right\rangle$ instead of performing an extensive series of numerical simulations. Namely, it is possible to compute the $\mu\left(\left[k_{t-1}, k_{t}, k_{t+1}\right]\right)$ and respectively the $\mu\left(\left[k_{t-2}, k_{t-1}, k_{t}, k_{t+1}\right]\right)$ on the basis of the meso chain $(\tilde{\mathbf{X}}, \tilde{P})$ which in turn is a loss-less description of the microscopic system (see Fig. 5.12).

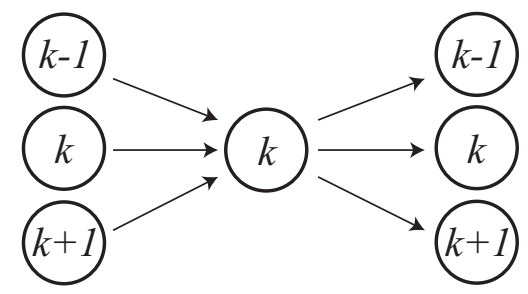

Figure 5.16: Possible paths of length 3 through $X_{k}$.

Let us consider that for the cylinders of length 3. As noted above, the grammar $\mathfrak{G}_{3}$ of the system is determined by the fact that $\left|k_{t}-k_{t-1}\right| \leq 1$ and

\footnotetext{
${ }^{2}$ In real computations, one always has to restrict to finite histories. Görnerup and Jacobi (2008) compute $\left\langle I_{2}\right\rangle=\Delta H_{2}-\Delta H_{4}$ which means that they consider cylinders up to length four $\left[k_{t-2}, k_{t-1}, k_{t}, k_{t+1}\right]$ in their Markovianity test.
} 

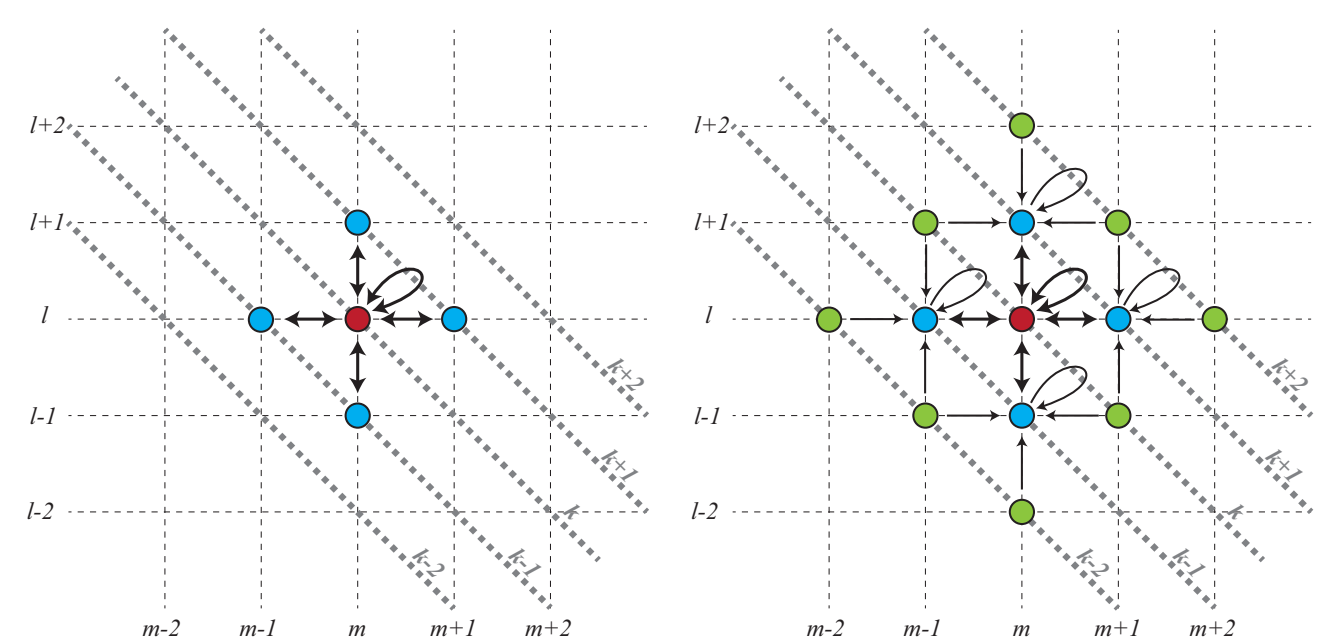

Figure 5.17: Illustration of the possible paths for one $\tilde{X}_{m, l}$ with $m+l=k$ for cylinders of length three (l.h.s) and four (r.h.s). An arrow indicates whether or not one state can be followed by another in a sequence.

$\left|k_{t+1}-k_{t}\right| \leq 1$. Therefore, as illustrated in Fig. 5.16, for any $k_{t}=k$ with $0<k<N$ there are nine possible paths $\left[k_{t-1}, k, k_{t+1}\right]$ and for $k=0$ and $k=N$ there are respectively four paths. In order to compute the probability of a certain macro path, say $[p, k, f] p$ for past and $f$ for future, we have to sum over all meso level paths that contribute to the given macro path. Let us denote a meso level path as $\left[\left(m_{p} l_{p}\right),(m l),\left(m_{f} l_{f}\right)\right]$ with $m_{p}+l_{p}=p, m+l=k$ and $m_{f}+l_{f}=f$. Its probability is given by

$$
\mu\left(\left[\left(m_{p} l_{p}\right),(m l),\left(m_{f} l_{f}\right)\right]\right)=\tilde{\pi}_{m_{p}, l_{p}} \tilde{P}\left(\tilde{X}_{m_{p}, l_{p}}, \tilde{X}_{m, l}\right) \tilde{P}\left(\tilde{X}_{m, l}, \tilde{X}_{m_{f}, l_{f}}\right) .
$$

The 1.h.s. in Fig. 5.17 illustrates the possible paths for one $\tilde{X}_{m, l}$ with $m+l=k$. Notice that for a given macro state $X_{k}$ there are $k+1$ meso states if $k \leq M$ and respectively $N-k+1$ meso states for $k>M$ (these numbers are for the case $M=L$ with $M+L=N)$. In the case sequences of length three are considered, the situation is still quite clear. For instance, a macro path $[k-1, k, k+1]$ can be realized in four different ways for each ${ }^{3}$ $\tilde{X}_{m, l}$ with $m+l=k$ :

$$
\begin{aligned}
& {[(m-1 l),(m l),(m+1 l)]} \\
& {[(m-1 l),(m l),(m l+1)]} \\
& {[(m l-1),(m l),(m+1 l)]} \\
& {[(m l-1),(m l),(m l+1)]}
\end{aligned}
$$

\footnotetext{
${ }^{3}$ Notice that the number of possibilities reduces at the corners or borders of the meso chain whenever $m=0$ or $l=0$.
} 
The same reasoning can be applied to derive the probabilities for cylinders of length four even though the situation becomes slightly more complicated, as illustrated on the r.h.s. of Fig. 5.17.

On the basis of the probabilities of blocks of length three and four respectively, the computation of the Markovianity measures $\left\langle I_{1}\right\rangle=\Delta H_{2}-\Delta H_{3}$ and $\left\langle I_{2}\right\rangle=\Delta H_{2}-\Delta H_{4}$ is straightforward. All that is needed is to compute the respective block entropies. Fig. 5.18 shows $\left\langle I_{1}\right\rangle$ (dashed curves) and $\left\langle I_{2}\right\rangle$ (solid curves) as a function of the coupling between the two communities $r$ for a system of $N=100$ agents $(M=L=50)$. The different curves represent various different contrarian rates $p$ from 0.001 to 0.05 . Notice the log-linear scaling of the figure.

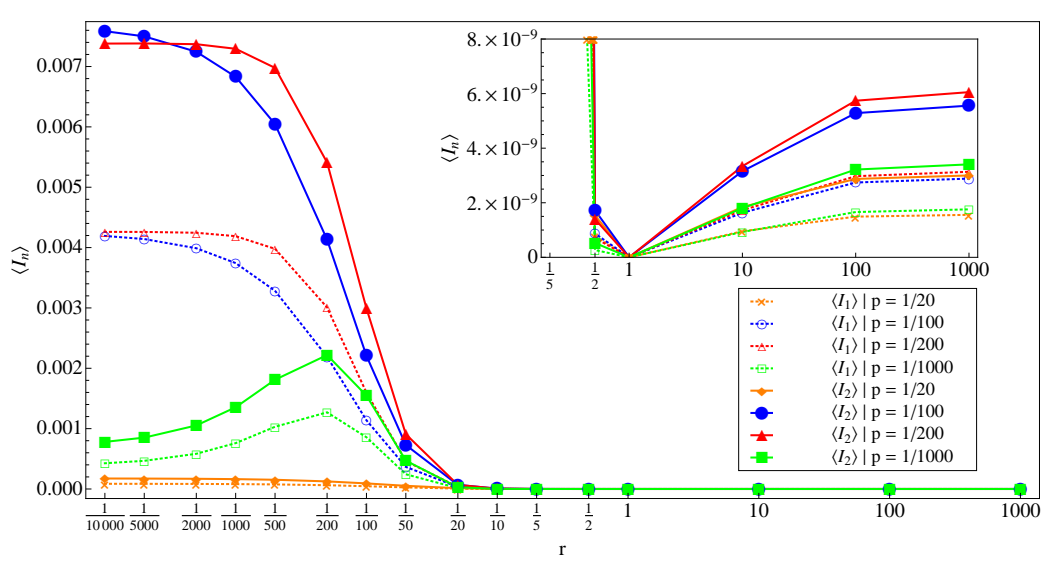

Figure 5.18: $\left\langle I_{1}\right\rangle$ (dashed curves) and $\left\langle I_{2}\right\rangle$ (solid curves) as a function of the coupling between the two communities $r$ for a system of $N=100$ agents. The different curves represent various different contrarian rates $p$ from 0.05 to 0.001 , see legend.

What becomes clear in Fig. 5.18, first of all, is that the deviation from Markovianity is most significant for small inter-community couplings. This means, in the reading of Görnerup and Jacobi (2008), that the additional information about the future state (beyond that given by the present) provided by pasts of length $n$ is larger than zero if $r$ becomes small. In general and not surprisingly, $\left\langle I_{2}\right\rangle>\left\langle I_{1}\right\rangle$ which means that both the first and the second outcome before the present provide a considerable amount of information. In fact, the numbers indicate that the first and the second step into the past contribute in almost the same way. Noteworthy, the two measures $\left\langle I_{1}\right\rangle$ and $\left\langle I_{2}\right\rangle$ behave in the same way from the qualitative point of view which suggests that the computationally less expensive $\left\langle I_{1}\right\rangle$ can be well-suited for the general Markovianity test.

The inset in Fig. 5.18 shows the situation for values around $r=1$ (homogeneous mixing) as well as $r>1$. As we would expect by the strong 
lumpability of homogeneous mixing, $\left\langle I_{1}\right\rangle$ and $\left\langle I_{2}\right\rangle$ are effectively zero (order $10^{-17}$ ) in the case $r=1$. Also if the inter-community coupling becomes larger than the coupling within communities (a situation that resembles a bipartite graph) $\left\langle I_{1}\right\rangle$ and $\left\langle I_{2}\right\rangle$ are very small which indicates that a Markovian macro description (that is, ideal aggregation) describes well these situations.

Finally, we notice in Fig. 5.18 that the measures do not generally increase monotonically with a decreasing ratio $r$ which is most obvious for the example with a very small $p=1 / 1000$ (green curves). This is somewhat unexpected and it indicates the existence of certain parameter constellations at which macroscopic complexity (for this is how non-Markovianity may be read) is maximized. To obtain a better understanding of this behavior, the measures $\left\langle I_{1}\right\rangle$ and $\left\langle I_{2}\right\rangle$ are plot in Fig. 5.19 as a function of the contrarian rate $p$. Notice again the log-linear scaling of the plot.

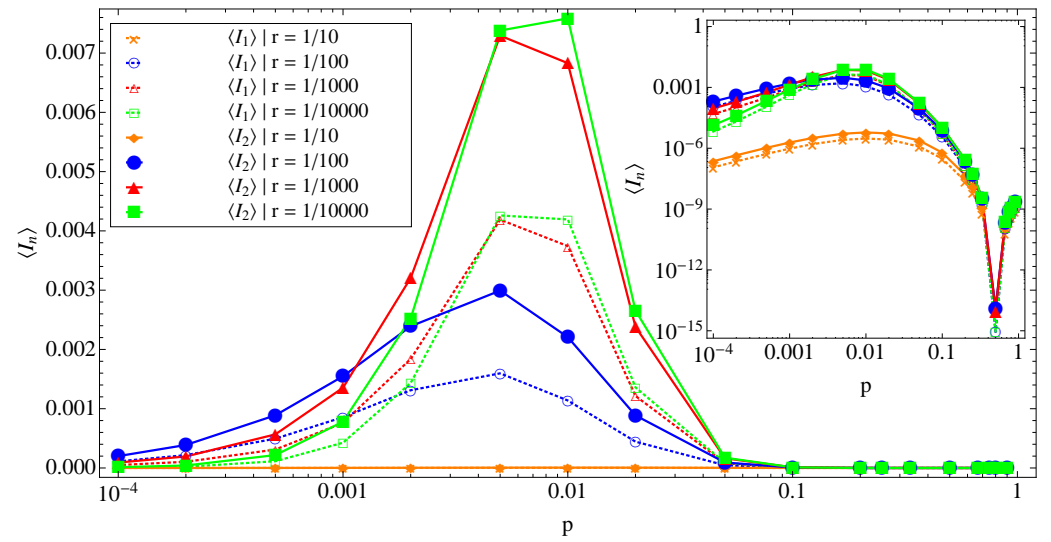

Figure 5.19: $\left\langle I_{1}\right\rangle$ and $\left\langle I_{2}\right\rangle$ as a function of the contrarian rate $p$ for various coupling ratios $r$ and a system of $M=L=50$.

It becomes clear that there is a strong and non-trivial dependence of the Markovianity measures on the contrarian rate $p$. Namely, $\left\langle I_{1}\right\rangle$ and $\left\langle I_{2}\right\rangle$ are very small if $p$ is relatively large but they are also relatively small if $p$ becomes very small. There is a parameter regime in between in which deviations from Markovianity become most significant. Notice that in the inset of Fig. 5.19 the same curves are shown on a double-logarithmic scale. This shows, first, that $\left\langle I_{1}\right\rangle$ and $\left\langle I_{2}\right\rangle$ for very small $p$ are still significantly larger compared to the case of relatively large $p$ (say $p>0.1$ ). Secondly, we observe that $\left\langle I_{1}\right\rangle$ and $\left\langle I_{2}\right\rangle$ actually vanish for $p=1 / 2$. As discussed in the previous section, the reason for that is the strong lumpability of the two-community CVM whenever $p=1 / 2$.

Finally, a detailed picture of the dependence of $\left\langle I_{n}\right\rangle$ on the contrarian rate is provided in Fig. 5.20. The plot compares the cases $r=1 / 100$ and $r=1 / 1000$ in order to show that the peak in the $\left\langle I_{n}\right\rangle$ depend also on $r$. 
For the interpretation of this behavior, notice that the $p$ at which deviations from Markovianity become largest, lie precisely in the parameter interval in which switching times between the two complete consensus states become minimal. Compare Fig. 5.4 in Sec. 5.2.3.

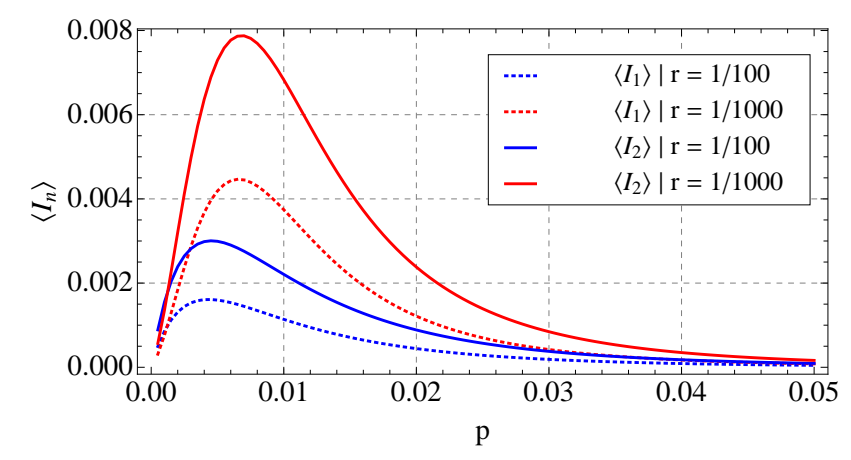

Figure 5.20: Detailed picture of the dependence of $\left\langle I_{n}\right\rangle$ on the contrarian rate. Blue curves correspond to $r=1 / 100$ and red curves to $r=1 / 1000$. In the first case the peak is at around $p \approx 0.05$, in the latter at $p \approx 0.065$.

\subsection{Discussion}

This chapter has provided an analysis of the CVM on the complete and the two-community graph. Based on the previous chapters, higher-level Markov chain descriptions have been derived and allow a detailed understanding of the two cases. A large contrarian rate $p$ leads to a process which fluctuates around the states with approximately the same number of black and white agents, the fifty-fifty situation $k=N / 2$ being the most probable observation. This is true for homogeneous mixing as well as for the two-community model. However, if $p$ is small, a significant difference between the two topologies emerges as the coupling between the two communities becomes weaker. On the complete graph the population is almost uniform for long periods of time, but due to the random perturbations introduced by the contrarian rule there are rare transitions between the two consensus profiles. On the community graph, an effect of local alignment is observed in addition to that, because the system is likely to approach a meta-stable state of intracommunity consensus but inter-community polarization.

A particular focus of this chapter has been on the effect of inhomogeneities in the interaction topology on the stationary behavior. In this regard, the two-community CVM served as a suitable scenario to assess the macroscopic effects introduced by a slight microscopic heterogeneity. Namely, homogeneous mixing compatible with the usual way of aggregation over all agents leads to a random walk on the line with $N+1=O(N)$ 
states whereas the two-community model leads to a random walk on a $2 \mathrm{D}$ lattice with $O\left(N^{2}\right)$ states. As the latter is a proper refinement of the former this gives us means to study the relation between the two coarse-grainings in a Markov chain setting. In this regard, this chapter has made visible the reasons for which lumpability fails, even in its weaker form (Sec. 5.4.4). And it has also provided a first analysis of the macroscopic memory effects that are introduced by heterogeneous interaction structures.

There are various issues that deserve further discussion. For instance, is the emergence of memory in the transition from the micro to the macro level a useful characterization for the complexity of a certain system? We shall discuss this point in the next chapter.

To finish this chapter, I would like to mention the possibility of applying the arguments developed throughout this chapter to the case of models with absorbing states as, for instance, the pure VM $(p=0)$. In that case, the quasi-stationary distribution (see Darroch and Seneta, 1965) takes the role of $\hat{\pi}$ or respectively $\tilde{\pi}$ in the construction of an ideal aggregate and the computation of cylinder measures. One interesting issue to be addressed in this regard is to reconsider the question of weak lumpability for the VM. Finally, to understand how microscopic heterogeneity and macroscopic complexity are related, numerical experiments with different network topologies are another promising way to continue the analysis started in this chapter. 


\section{AgGregation And EmergenCE: A SYNTHESIS}

This chapter is an attempt to synthesize some of the thoughts that have been developed throughout this work. While more specific problems concerning the methods developed in Chapter 3,4 and 5, their limitations and possible generalizations have been discussed at the end of each chapter, this chapter aims at a synthetic view on how this work may contribute to an important aspect of complexity science.

\subsection{The Computational View on Emergence}

To my point of view, one of the most important contributions is the perspective that a Markov chain theory of aggregation for ABMs may provide on emergence and emergent phenomena. Namely, as explained in the second Chapter, ABMs along with cellular automata (CA), genetic algorithms and other related computational tools play an increasingly important role in the philosophical discussions around emergence. Interestingly enough, some philosophers advocate a position which makes use of computational models as a playground to address fundamental questions of emergence (Bedau, 1997; Huneman and Humphreys, 2008; Humphreys, 2008, among others). Questions about the relation of these artificial model environments to real phenomena are not ignored, but considered as an independent issue which is actually part of another debate. ${ }^{1}$ In this way, the philosophical controversy that usually comes with the term »emergence« (see O'Connor and Wong, 2012) is circumvented to some extend. This thesis has been following a similar tradition, as the models dealt with are also very simple and the focus has been on the method rather than on empirical adequacy.

Let us quote from the introduction to a special issue on $\gg$ Dynamical

\footnotetext{
${ }^{1}$ To me, this position has been most clearly articulated by Humphreys (2012) in a talk given at the 2012 DPG Tagung. See Symons (2008) for a critical account on the possible contributions of this approach to the »metaphysical« problem of emergence.
} 
Emergence and Computation « in Minds \& Machines (2008) Volume 18 (Huneman and Humphreys, 2008, 426) in order to point out in what regard our approach may contribute to these discussions:

$\gg$ Other problems appear concerning the criteria for those types of emergence. Up to this point, the link between criteria for emergence and simulation can be put in this way: Is the unavoidability of simulation, or the incompressibility of computing the final state, a sufficient criterion for diachronic emergence? And is this criterion epistemological or ontological, i.e. does this criterion lead to a description of emergence which depends on our cognitive abilities, or does it provide an essential characterization of the phenomenon? Yet how can we prove that the unpredictability, except by simulations, picks out an objective property of a system, and is not a peculiar limitation of our cognitive abilities? What kind of further criteria do we need if we want a more finegrained classification of diachronic emergences? Can we derive such a classification from a typology of simulations? «

By means of a formulation of computational models as Markov chains we may shed new light some of these questions. First of all, a Markov chain formulation at the micro level challenges a definition that makes strong reference to simulation (as by »unavoidability of simulation «, ibid., 426) or likewise to analytical unpredictability (»emergent phenomena [...] as unpredictable in an analytical way from the equations of the system «, ibid., 425) of dynamical emergent phenomena. All criteria with reference to our capabilities of dealing analytically with the problems are prone to mere epistemological accounts of emergence and will not lead to an ontology of dynamical emergences. On the other hand, the possibility or impossibility to lump sets of states into a new chain at the macro level - that is, to compress the process - indicates that the dynamical incompressibility of a computational process might indeed form an appropriate criterion for dynamical emergence. Though both »the unavoidability of simulations « and »the incompressibility of computing the final state « capture the essential aspect of computational emergence, dynamical incompressibility seems to be the more sophisticated argument.

This is not least so because it should be in the relation between the micro and the macro where appropriate criteria for emergence may be found. In fact, the analysis of the micro-macro link in simple computational models as the voter model (VM), leads naturally to some of the conditions for nonaggregativity (and therefore emergence) proposed in Wimsatt $(1986,2006 \mathrm{a}$ ). Wimsatt's aggregativity conditions, most importantly inter-substitution of parts, are derived in a probabilistic framework to be the mathematical conditions that have to be met so that the micro process is lumpable. This 
work suggests to base a proper differentiation between emergent phenomena and non-emergent features on the question whether they meet certain aggregativity conditions, notably, in a dynamical setting.

\subsection{Challenging Definitions of Weak Emergence}

\subsubsection{Unavoidability of Simulations}

Bedau (2003) defines weak emergence in the following way:

»Assume that $\mathrm{P}$ is a nominally emergent property possessed by some locally reducible system $\mathrm{S}$. Then $\mathrm{P}$ is weakly emergent if and only if $\mathrm{P}$ is derivable from all of S's micro facts but only by simulation. «(p.15)

We have shown in Chapter 3 that many computational models can be formalized as random walks on more or less regular graphs. The configuration space $\boldsymbol{\Sigma}$ of the model is the set of all possible configurations of the simulation and for a considerable class of models we are able to derive explicitly the transition probabilities between all these configurations, among them models that are widely considered to give rise to weakly emergent patterns. As a result we have at hand an analytical description of the computational model that allows us, in principle, to understand all the dynamical processes, final states or stationary distributions without a need of performing simulations. For instance, if the emergent property $\mathrm{P}$ is a stable pattern which the model converges to, this corresponds to an absorbing state in the Markov chain formulation. We are able to compute the probability with which the random walk will end up in that (and in any alternative absorbing) state, and, even without any computation, we know that convergence happens in finite time. In principle, therefore, simulations are avoidable.

However, analytical predictability of the model results are challenged by the exponential increase of the dimension of the Markov chain description as the number of elements increases. In practice, therefore, the problem remains unpredictable at this level of description and there is no other choice than performing simulations. The resulting unavoidability of simulations is then essentially due to the cognitive difficulties to derive and calculate the explicit Markov chains for some more complicated and bigger models and the technical impossibility to handle matrices of that size on current computer systems. Seen in this way, a criterion based on the analytical unpredictability of a model of emergent phenomena is an epistemological criterion and leads to an epistemological account of emergence. One could also object that even if an analytical description in form of a micro chain $(\Sigma, \hat{P})$ is found, one still has to »simulate « the chain by applying the transition matrix to a certain initial distribution of interest. One still has to rely on all »micro facts «. On the other hand, however, certain properties of the chain (as, for instance, 
convergence to an absorbing state in finite time) can be assessed without any computation or reduction to a simpler description (see also Izquierdo et al. (2009) who mention some of these properties for rather complex models).

To my point of view, criteria for emergent behavior should not be defined with reference to our capabilities of dealing with the respective problem using analytical methods. It seems that this view originates from an implicit tendency to assume that analytical descriptions are differential equation describing the problem at an aggregate macro level, disregarding the possibility of an analytical formulation at the micro level. For our mathematical tools are under constant development and because we cannot foresee whether new methods for the analysis of complex systems allow analytical predictions in the future, any such criterion makes - by the very construction - emergence a purely epistemological question and rules out any hypothesis about emergent phenomena in an ontological sense.

\subsubsection{Computational Incompressibility}

Very often, the necessity of performing simulation has been related to the impossibility to reduce the problem to a simpler one by deriving $\gg$ directly « a macroscopic description of the problem. In his 2003 paper (Bedau, 2003), from which the above definition has been cited, Bedau himself equates weak emergence and computational irreducibility (»Computational irreducibility - that is, weak emergence«, p.18) with reference to the well-known work on CA by Wolfram (1994). Here we will use the term computational incompressibility which has been used by Huneman and Humphreys (2008); Humphreys (2008) especially in the context of diachronic (i.e., dynamical) emergence.

While preserving the essence of the argument, the concept of dynamical incompressibility provides a definition of weak emergence which is not by construction an epistemological one, because the question whether a process or a model can be compressed is truly a property of the process. It is certainly closely related to analytical predictability (and thus unavoidability of simulations), namely, when a macro description in form of, for instance, a differential equation is considered as the reference analytical formulation to which the micro process can be reduced. According to this view, a property is weakly emergent if the process leading to the generation of it is computationally incompressible (Humphreys, 2008). Markov chain aggregation provides precise arguments for whether such a complexity reduction is feasible. The transition from micro to macro corresponds, in essence, to a compression of subsets of micro configurations into macro states and in this way Markov chain aggregation - that is, lumpability - operationalizes the concept of computational compressibility. The question of dynamical incompressibility (and therefore, the question of emergence) is then understood as whether this transition from micro to macro is with or without loss of information.

More precisely, we have seen in the third chapter (and other have before, 
e.g., Shalizi and Moore, 2003) that any system property defines a partition of the state space of the micro chain. Then, there are necessary and sufficient conditions (Kemeny and Snell, 1976, Thm. 6.3.2) for the process projected onto this partition to be again a Markov chain, that is, a macro description of the process which contains all information about the system (in the true sense of information, see Chapter 5, Sec. 5.4.5). If we want to keep up a definition of emergence on the basis of dynamical incompressibility, we could put forth the following definition: a system property $P$ is emergent if the system is lumpable with respect to the macro description defined by $P$.

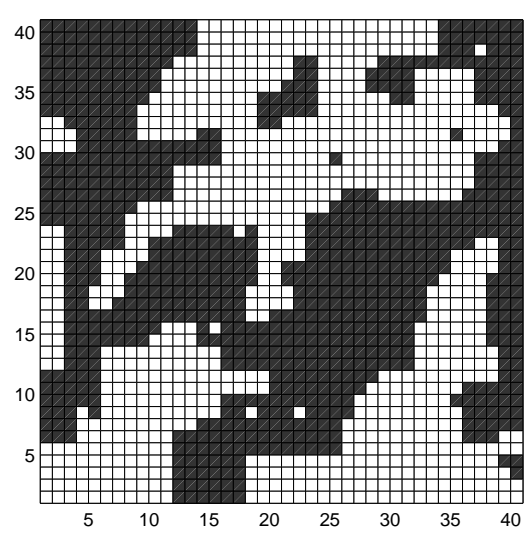

Figure 6.1: An instance of emergent »spatial alignment « in a simple segregation model.

At its core, this Markov chain approach to emergence deals with a type of emergent behavior that might be called »process emergence«. It relates processes at a micro level, typically those arising from simple local rules, to processes at a macro level typically obtained by averaging or aggregating certain properties. The focus is not on the emergence of a stable pattern or a higher level structure that »results « from a simulation, it is rather on establishing a relation between processes on the different levels. In fact, »resultant « stable or recurring macro properties are often reflected, in the process point of view, as absorbing or meta-stable states with a high stationary probability (or classes of those).

Notice that this view on computational incompressibility is closely related to the information-theoretic approaches to measure complexity and emergence as developed, for instance, in Grassberger (1986); Lindgren and Nordahl (1988); Crutchfield and Young (1989); Crutchfield and Shalizi (1999); Shalizi and Crutchfield (2001); Shalizi et al. (2004); Ball et al. (2010); Gmeiner (2013) and that a construction based on Markovianity is in fact a special case of these more general approaches. 
I briefly comment on some possible objections to such a definition. The first point is in fact a general difficulty of defining a property as weakly emergent if the processes leading to it are computationally incompressible. Consider, as an example, the emergence of $»$ spatial alignment « in voter-like and segregation models. See Fig. 6.1 for an instance of »spatial alignment « that emerged in a simple version of Schelling's segregation model (Schelling, 1971). In the two-community model (for the VM as well as for the CVM) we observe the emergence of »spatial alignment « or segregation in the form of intra-community consensus and inter-community polarization (Chapter 4 and 5). However, the process with $2^{(M+L)}$ states at the micro level ( $M$ and $L$ being the community sizes) is compressible to a description of size $(M+1) \times(L+1)$ which is an essential reduction. On the other hand, we have seen that on the ring topology (and similarly for the grid, see Fig. 6.1) the number of states that are needed to obtain a Markovian macro description is larger than $2^{N} / N$ states which cannot be considered $\gg$ essentially simpler than the microscopic computational process by which the system's behavior is generated «(Bedau, 2003, 18). This raises the question whether $»$ spatial alignment « is emergent in one case but not in the other. In the same way one could ask whether the emergence of complete consensus in the VM (which often comes as a surprise to people unacquainted with this kind of models) is emergent if the model is run on a complex network but not emergent if it is run on the complete graph.

Secondly, a definition based on Markov chain aggregation requires to rigorously define the system property corresponding to a certain emergent feature. On the one hand, this points at a deficiency of existing definitions which are usually not explicit on the property and the processes related to it. On the other hand, it may not always by easy to rigorously define the system property corresponding to a pattern of interest even if the feature catches the observer's eye immediately when looking at the outcome of a simulation. In the above example, one could argue that it is not so clear to which micro configurations an observer would ascribe the property of »spatial alignment «. However, there are clearly ways to measure the amount of alignment, for instance, by taking into account the number of aligned neighbors. The respective measure defines again a partition on the space of micro configuration. In fact, pair approximation and approximate mean field theories (Gleeson, 2013, and references therein) derive systems of differential equations in a similar way. This point also brings into the discussion the role of the observer and the idea that emergence comes in degrees (Humphreys, 2008). In this regard, the non-Markovianity measure used in Sec. 5.4.5 could be a way to $»$ measure emergence $\ll$.

Finally, a definition based on exact lumpability may be too unrestrictive on the one and not widely applicable on the other hand. Too unrestrictive because in Markov chains in general lumpability is the exception rather than the rule Chazottes and Ugalde (2003); Gurvits and Ledoux (2005). 
Not applicable to important cases, because the full specification of a micro chain and the projection operator may become very complicated for more sophisticated computational models. Even for classical CA with synchronous update, which are widely used in the context of computational emergence, such a specification is by no means straightforward. ${ }^{2}$

\subsection{From Computational Incompressibility to Non- Aggregativity}

Emergence is a concept that relates parts and wholes. Dynamical (or diachronic) emergence is a concept that relates processes on the level of parts to processes on the level of the whole. In our setting, processes at the micro level are processes of change of certain attributes of a set of agents arising from simple local interaction rules. The macro-level process is obtained by the observation or measurement of certain indicators that inform us about the global state of the entire agent population. Typically (though probably not in every case) such a measurement corresponds to an averaging or aggregation of certain agent properties or of properties of the links between agents (i.e., link-between-parts properties). In the VM, for instance, considering the frequency of the different attributes in the population is a one of the simplest forms of aggregation. In the sugarscape model (Epstein and Axtell, 1996), to make another example, the number of agents in the environment or the average amount of sugar they carry are similarly aggregative measures. Examples of system properties obtained by aggregation over links-betweenparts properties include the number of unaligned neighbors (active bonds) in the $\mathrm{VM}$ or, in a more complex setting, the time evolution of density or other structural indicators of a network that co-evolves in the simulation.

Hence, the link between the level of parts and the whole is realized in form of system properties and the typical way to define them is via some form of aggregation. Emergence occurs when the dynamical evolution of the system property ceases to be Markovian. That is, when a Markovian process at the micro-level gives rise to a non-Markovian process at the macro-level. In other words, when aggregation fails to capture all the dynamical details of the micro-level process.

In this reading, emergence and »aggregativity « define a fundamental dichotomy between emergent and non-emergent phenomena; a view that, to the authors knowledge, has first been expressed by Wimsatt (Wimsatt, 1986, 2006a,b):

»Aggregativity, a claim that "the whole is nothing more than the sum of its parts", is the proper opposite to emergence « (Wimsatt, $2006 \mathrm{~b}, 4)$

\footnotetext{
${ }^{2}$ Notice that CA with asynchronous stochastic update, to the contrary, belong to the class of single-step dynamics the specification of which has been the subject of this thesis.
} 
Calling for an account of emergence consistent with reductionism, Wimsatt starts out from the question of aggregativity: $»$ When intuitively is a system "more than the sum of its parts"? (Wimsatt, 2006a, 673). He states four criteria that a system property in relation with the parts of that system must fulfill to be a fully aggregative property (ibid, 676):

1. invariance of the system property under operations rearranging the parts (inter-substitution),

2. qualitative similarity of the system property under addition or subtraction of parts (size scaling),

3. invariance of the system property under operations involving decomposition and re-aggregation of parts (decomposition and re-aggregation),

4. there are no cooperative or inhibitory interactions among the parts which affect the system property (linearity).

Noteworthy (see Chapter 2), such an account of emergence requires to be explicit on the definition of the system property at question, for one and the same system might be aggregative for one but emergent for another property. This is very similar to lumpability which only makes sense (it is actually only defined) in relation to a certain partition of the state space.

With the analysis of the VM in Chapter 3 we recover the first of Wimsatt's criteria. ${ }^{3}$ Actually we came to the conclusion that aggregativity depends exclusively on the invariance of the system property with respect to the inter-substitution of parts. If the system property (the projection from micro to macro) is defined in accordance with the symmetries of the agent relations, it will be an aggregative measure and correctly describe the evolution of the system (Chapter 4). It might be that models with more complicated interaction mechanisms will require a closer inspection of the other three criteria. On the other hand, Wimsatt (2006a) notes that $»[t]$ hese conditions are not independent of one another.«(p.675). Moreover, for all models in which the local transitions depend only on the neighborhood configurations, invariance of agent relations with respect to agent permutations is sufficient for lumpability and ensures compressibility of the process.

Finally, in order to stress that we are dealing with dynamical emergence, let me mention a subtlety when applying Wimsatt's arguments to the VM. One could actually argue that all of the aggregativity criteria are met by a macro formulation in terms of attribute frequencies $h(\mathbf{x})$ (Hamming weight) independent of the agent network. Namely, at each instance, that is, for each single micro configuration, the system property $h(\mathbf{x})$ satisfies all of

\footnotetext{
${ }^{3}$ One of Wimsatt's main concerns is to show that in natural phenomena full aggregativity (present if all four conditions are satisfied) is the exception rather than the rule and that in many models - including voter-like models of population genetics - the use of aggregative procedures is unjustified (Wimsatt, 2006a).
} 
Wimsatt's aggregativity conditions. It is invariant with respect to alternative arrangements of the agents. Qualitative similarity (even an obvious quantitative relation) after addition or subtraction of parts is also satisfied. Thirdly, given any configuration $\mathbf{x}$, it is possible to decompose the system in an arbitrary way, compute $h(\mathbf{x})$ for subsets of agents, and re-aggregate (sum up) the measures for the different subsets. Finally, $h(\mathbf{x})$ is also invariant with respect to non-symmetric interaction relation, just because it only takes into account node (agent) properties. Noteworthy, this works for any agent network.

The answer to this simple puzzle resides in the shift from a synchronic to a diachronic - that is, process-based - perspective. A dynamical argument is also presented in Wimsatt (2006a) to illustrate aggregation failures in classical population genetics, but the distinction between a synchronic and a diachronic emergence is not always clear. It might even be the case (but this certainly deserves a further inspection) that the aggregativity criteria with reference to dynamical operations (most importantly criteria 4) appear redundant if an explicit process perspective is taken. Namely, in the context of Markov chain aggregation the system property on which aggregativity condition must be assessed is not the attribute frequency, but rather the transition probability from one frequency level to the other. From the point of view of lumpability this is obvious, but it is not, in general, from the point of view of emergence.

To sum up, a definition of emergence in terms of lumpability provides a link between the concept of dynamical incompressibility and Wimsatt's notion of non-aggregativity. It shares an intrinsic emphasis on processes with the former and with the latter a clear concept of system property as well as the idea that emergence and aggregativity define a dichotomy between emergent and non-emergent phenomena. 


\section{CONCLUSION}

This thesis introduces a Markov chain approach for the analysis of agentbased models (ABMs). It provides a general framework of aggregation in agent-based and related computational models by making use of Markov chain aggregation and lumpability theory in order to link between the microlevel dynamical behavior and higher-level processes defined by macroscopic observables. The starting point is a formal representation of a class of ABMs as Markov chains - so-called micro chains - obtained by considering the set of all possible agent configurations as the state space of a huge Markov chain. This allows for the application of the theory of Markov chain aggregation namely, lumpability - in order to reduce the state space of the models and relate microscopic descriptions to a macroscopic formulation of interest. In some cases, the aggregation is without loss of information and the macro chain can be used to compute several stationary and transient characteristics of the models. In general, however, a certain amount of macroscopic complexity is introduced by the transition from the micro level to a favored macro description which is a fingerprint of emergence in agent-based computational models.

\section{ABMs are Markov chains.}

While Markov chains represent a relatively simple form of a stochastic process, ABMs put a strong emphasis on heterogeneity and social interactions. Nevertheless, most ABMs are from the formal point of view Markov chains. Intuitively, this might be clear by the fact that ABMs usually come in form of a computer program which takes a certain initial population of agents as an input and iteratively applies an algorithm to evolve the agent population from one time step to the other. In order to formally represent such an iterative process as a Markov chain, a single state of the chain must be conceived of as a possible configuration of the entire system and contain all the dynamical variables and microscopic details - agent attributes, their connectivity structure, state of the environment etc. - an idea that this thesis has borrowed from Izquierdo et al. (2009). A rigorous proof of 
the Markovianity of ABMs is not always straightforward. However, the micro process is a Markov chain whenever the iteration it implements can be understood as a (time-independent) stochastic choice out of a set of deterministic options. In this respect, the random mapping representation of a Markov process helps to understand the role of the collection of (deterministic) dynamical rules used in the model from one side and of the probability distribution $\omega$ governing the sequential choice of the dynamical rule used to update the system at each time step from the other side.

2. A class of ABMs that we have called single-step models give rise to random walks on regular graphs.

Moreover, for a class of models which we have referred to as single-step dynamics, it is possible to derive explicitly the transition probabilities $\hat{P}$ in terms of the update function $\mathbf{u}$ and the probability distribution $\omega$. Due to a sequential update mechanism in which an agent along with a set of neighbors is chosen and the state of that agent is updated as a function of the neighborhood configuration, non-zero transition probabilities are possible only between configurations that differ in at most on element (one agent). This characterizes ABMs as random walks on regular graphs. Namely, in a system with $N$ agents each of which may be in one out of $\delta$ states, the set $\boldsymbol{\Sigma}$ of all agent configurations is the set of strings of length $N$ of $\delta$ possible symbols. Under sequential update of only one agent at a time, transitions are possible only between adjacent strings so that the maximal $»$ grammar « of such a system is the Hamming graph $H(N, \delta)$. However, a completely regular walk on $H(N, \delta)$ with non-zero transition probabilities between all adjacent configurations is realized only if no constraints act in the system. In particular, as will be resumed below, if the interaction probabilities and therewith the distribution $\omega$ are constrained, for instance, by an underlying interaction network, the structure of the micro chain becomes more and more irregular. The same is true for other interaction constraints such as assortativity or bounded confidence.

3. Regularity implies dynamical redundancy and therefore the possibility of state space reduction.

Nevertheless, the approach to ABMs as random walks on more or less regular graphs hints at the possibility of reducing the state space of the micro chain by exploiting systematically the dynamical symmetries that an ABM gives rise to. Namely, the existence of non-trivial automorphisms of the micro chain tells us that certain sets of micro configurations can be interchanged without changing the probability structure of the random walk. These sets of micro states can be aggregated or lumped into a single macro state and the resulting macro-level process is still a Markov chain. In Markov chain theory, 
such a state space reduction by which no information about the dynamical behavior is lost is known as lumpability.

4. Macro observations and system properties induce state space partitions and reductions.

There is another way of looking at state space reductions or aggregation which is particularly relevant in the study of ABMs. Namely, any observable of the system naturally defines a many-to-one relation by which sets of micro configurations with the same observable value are aggregated into the same macro state. In other words, tracking the time evolution of a model in terms of a system property or order parameter $\phi$ that informs us about the global state of the system corresponds to a projection $\Pi$ of the micro chain onto a partition $\mathbf{X}$ of the space of micro configurations $\boldsymbol{\Sigma}$. Vice versa, any projection map $\Pi$ from $\boldsymbol{\Sigma}$ to $\mathbf{X}$ defines an observable $\phi$ with values in the image set $\mathbf{X}$ that are in complete correspondence with a classification based on an observable property of the system. These two ways of describing the construction of macro-dynamics are equivalent and the choice of one or the other point of view is just a matter of taste.

5. A macro observation defines a Markov process if it is compatible with the symmetries of the micro chain.

6. Vice versa, the symmetries of the micro chain induce a partition with respect to which the process is lumpable

The main question that this thesis has been concerned with is about the conditions on the microscopic system $(\boldsymbol{\Sigma}, \hat{P})$ and the projection construction $(\Pi: \boldsymbol{\Sigma} \rightarrow \mathbf{X}$ or respectively $\phi)$ that have to be met in order to lead to a macro process that is still a Markov chain. The starting point has been Kemeny and Snell (1976), Thm. 6.3.2, in which necessary and sufficient condition for lumpability are provided. On that basis, a sufficient condition has been provided with Thm. 3.2.2 that relates the question of lumpability to the symmetries in the dynamical structure of the micro chain. Namely, to any partition $\mathbf{X}$ of $\boldsymbol{\Sigma}$ there is a transformation group $\mathcal{G}$ acting on $\boldsymbol{\Sigma}$ that generates $\mathbf{X}$ and Thm. 3.2.2 states that the micro process $(\hat{P}, \mathbf{\Sigma})$ is lumpable to a macro process $(\mathbf{X}, P)$, if $\hat{P}$ is symmetric with respect to $\mathcal{G}$. The automorphisms of the microscopic transition matrix (for $\mathcal{G} \subseteq \operatorname{Aut}(\hat{P})$ see Lemma 3.2.3) can therefore be used to construct a partition $(\mathbf{X})$ with respect to which the process is lumpable. In turn, an observation on the system will define a lumpable macro process if it is compatible with the symmetries of the micro chain.

7. In the voter model, homogeneous mixing is a prerequisite for lumpability with respect to aggregation over all agent attributes. 
This thesis has made extensive use of the voter model (VM) - one of the simplest $\mathrm{ABM}$ - to illustrate these points. In the binary VM each agent can be in two possible states $\square$ and $\boldsymbol{\square}$. At each time step, two agents (linked in the interaction network) are chosen at random with probability $\omega(i, j)$ and one of them copies the state of the other. From the microscopic perspective the binary VM is a random walk on the $N$-dimensional hypercube $(H(N, 2))$ and the Hamming weight $\phi(\mathbf{x})=h(\mathbf{x})$ (to maintain this terminology) of an agent configuration is the most typical macro level of observation. In effect, all micro configurations with the same Hamming weight are mapped into the same macro state which is a tremendous reduction from $2^{N}$ micro states to $N+1$ macro states. However, Chapter 3 has shown that the symmetries of the micro chain $\hat{P}$ are compatible with that level of observation only if the probability distribution $\omega$ is invariant with respect to all agent permutations. Markovianity at the macro level requires that the probability with which two agents are chosen $\omega(i, j)$ is equal for all agents pairs which renders homogeneous mixing a prerequisite for lumpability. The resulting process is known as Moran process (after Moran, 1958).

8. The use of Markov chain theory enables a complete characterization of the dynamical behavior of the VM with homogeneous mixing.

Throughout Chapter 3 the VM with homogeneous mixing and the resulting macro chain on $\mathbf{X}=\left(X_{0}, \ldots, X_{k}, \ldots X_{N}\right)$ with $h(\mathbf{x})=k$ has been discussed in detail. Due to the structure of the macro chain it has been possible to derive a closed-form expression for the fundamental matrix $\mathbf{F}$ for arbitrary $N$. Encoding the recurrence and hitting times of the system, this provides all the information about the mean quantities and variances of the transient dynamics in this model. Noteworthy, Markov chain theory allows for some computations that are not easy with other methods such as meanfield approaches. For instance, it is possible to characterize the convergence behavior of realizations that end up in one absorbing state independently from those that end up in the other one (Section 3.4.4). Moreover, the multi-state VM in which agents can adopt $\delta$ different states is shown to be reducible to the binary VM by a further lumping. However, only if the interaction is unconstrained in the sense that all agents and all attributes interact in the same way. In turn, if interactions are constrained by assortativity or bounded confidence this may lead to a stable pattern of polarization at the level of the entire population (Section 3.4).

9. Microscopic heterogeneity translates into dynamical irregularities in the micro chain and requires a refinement of the aggregation and the corresponding level of observation.

When inhomogeneities are introduced in the model, the symmetry conditions for lumpability in Thm. 3.2.2 (as well as Kemeny and Snell, 1976, 
Thm. 6.3.2) are no longer satisfied for the partition $\mathbf{X}$ induced by aggregation over all agents (i.e., by $h(\mathbf{x})$ ). However, Chapter 4 shows at the example of the VM that a refinement of the aggregation procedure is possible which is based entirely on the symmetries of the interaction probabilities $\omega$. Prop. 4.3.1 states that the automorphism of $\omega$ may be used to define a group $\mathcal{G}$ of bijections on $\boldsymbol{\Sigma}$ which generates a lumpable partition $\mathcal{M}_{\omega}$ of $\boldsymbol{\Sigma}$. Noteworthy, while Thm. 3.2.2 as well as common approaches to lumpability require the analysis of the $\delta^{N}$-dimensional micro chain, with Prop. 4.3.1 the result is stated in terms of the symmetries of a interaction network of size $N$. The most important implication of Prop. 4.3.1 is that the higher the amount of heterogeneity in the agent system, the lesser the coarse-graining that is possible if a Markovian description is desired to capture all the dynamical details of the micro process. In other words, the more constrained and heterogeneous the microscopic interaction probabilities and rules, the more irregular the micro process and the lower the chances to obtain a reasonable reduction by Markov chain aggregation.

10. Markov chain aggregation leads to solvable chains (only for) for »simple« population structures.

It is clear then that the exact aggregation by lumpability significantly reduces the number of states only if the interaction network that underlies the model possesses a lot of symmetries. This restricts the applicability of the method as a solution technique for ABMs to stylized situations as the leader-follower topology (Section 4.5) or the two-community model (Section 4.4 and 5.2.4). Nevertheless, even in those stylized situations interesting features can be observed. In the leader-follower system, the probability that the leader imposes its opinion on a follower population increases with the influence asymmetry between a leader and the followers but is independent of peer-to-peer processes among followers. A greater influence of the leader also accelerates the convergence process, however, this effect is undermined by a stronger peer-to-peer communication (Section 4.5). For the two-community $\mathrm{VM}$, in which a weak influence exists between two strongly connected groups, a general increase in convergence times is observed due to the existence of meta-stable states of intra-community consensus and inter-community polarization. This is also observed in the quasi-stationary distribution of the two-community VM.

Similar results are obtained for the contrarian VM (CVM) in which agents act in a contrarian way with a small probability $p$ (Chapter 5). An increasing contrarian rate leads to a process that is characterized more and more by random state flips independent of whether the agents are completely connected or organized in communities. As $p$ becomes smaller, topological effects become visible. On the complete graph the population is almost uniform for long periods of time, but due to the random perturbations introduced by the contrarian rule there are rare transitions between the two 
consensus profiles. Noteworthy, there is a characteristic $p$ at which the rate of switching becomes maximal (Section 5.2.2). On the two-community graph the system is likely to approach the states of inter-community polarization and remain there for quite some time. Such ordering behavior is also observed for other networks with a strong local structure (Section 5.3.2).

\section{Microscopic heterogeneity leads to macroscopic complexity.}

Another interpretation that Prop. 4.3.1 suggests is that microscopic heterogeneity introduces complexity at the macroscopic level. This idea has been taken up in Chapter 5 using the CVM as an example. If we decide to stay at the level of full aggregation over all agents $(h(\mathbf{x}))$ despite the fact that it is not compatible with the symmetries of the micro chain, the process obtained by this projection is no longer a Markov chain. This means that a certain amount of memory is introduced at the macroscopic level by the very way the system is observed. In the last part of Chapter 5, this divergence from Markovianity has been quantified in terms of the information that the past (before the present) contains about the future. The two-community CVM has served as a scenario in which these entities can be explicitly computed. Again, there is a characteristic contrarian rate $p$ at which deviations from Markovianity are maximal.

The method informs us in this way about the complexity of a system introduced by non-trivial interaction relations. Namely, the theory of Markov chain aggregation makes explicit statements about when a micro process is compressible to a certain macro-level description. This links non-lumpability to computational incompressibility, one of the key concepts in dynamical emergence (Bedau, 2003; Huneman and Humphreys, 2008, among others). Moreover, in the context of Markov chain aggregation, computational incompressibility becomes directly related to Wimsatt's notion of non-aggregativity (Wimsatt, 1986, 2006a), another important account of emergence. The argumentation in Chapter 6 suggests that deviations from Markovianity at the macro level can be understood as a fingerprint of dynamical emergence - and hence complexity - as the macroscopic process displays features that are not present in the micro level process.

The models used in this thesis are very simple and I would not claim that they are reasonable descriptions of real social phenomena. Their main purpose is to shed light on some fundamental mechanism of self-organizing systems. In this regard, I would like to emphasize the role that constraints on the agent behavior play regarding the aggregativity or reducibility of the models to a macro-level description. Even in those simple models, complex and heterogeneous interactions structures rule out completely the possibility of deriving a loss-less Markovian macro description which is sensitive to all dynamical details. Likewise do constraints on the interaction rules (as 
assortativity) necessitate the inclusion of more detail into a valid macro-level description in order to account for population effects (as polarization) that emerge from them. With their obvious limitations, the models used here do not allow for a direct generalization to more realistic cases, their treatment is only the first step in the stochastic analysis of the micro-macro link in social simulation. On the other hand, an increase in model complexity in more sophisticated ABMs comes often by introducing various levels or dimensions of agent heterogeneity, different types of agents with different rules, and a possibly variable environment. The macro patterns we observe in them are always the result of an adjustment of the constraints on and heterogeneities in the microscopic system and the fundamental mechanisms those that are at play also in the simpler models.

Nevertheless, the application of the ideas presented in this thesis to other ABMs is certainly an interesting issue for the future. Even if this has to be carefully considered model by model, a micro formulation in form of a micro chain will usually be possible (see Section 3.5). For more sophisticated ABMs, however, deriving an exact aggregate description of a size which allows for direct computations by the use of strong lumpability is not likely. On the other hand, there are nowadays powerful computational techniques to deal with large Markov chains, and interestingly, these methods are often based on approximate aggregations of the chains (Buchholz, 2006; Stewart, 2009; Touzene, 2013, among many others). In this context, the presented concepts might help in the analysis of the adequacy of such approximate techniques, and they may also shed light on the relation between approximate aggregations and the macroscopic measures they can be associated with.

The method is most directly applicable to models at use in socio-cultural dynamics, evolutionary graph theory as well as to stochastic cellular automata (CA). Regarding the first field, one interesting extension concerns simple forms of memory in the agent decisions such as agents that remember the states that they have already visited (Bornholdt et al., 2011) or by assuming that the probability of an agent to change its opinion decreases with the time it sticks to the current one (Stark et al., 2008). The macroscopic effects of these simple extensions are very interesting and encouraging for further analysis. Likewise, a more sophisticated modeling consists of coupling the individual agent dynamics with the macro dynamics and allow certain macro-structural properties to feed back onto the level of individual decisions. Such ingredients have been introduced into models of herd behavior in finance (Krause and Bornholdt, 2013) and they are also relevant in voting behavior (Caruso and Castorina, 2005).

The main question in evolutionary graph theory is how the population structure $\omega$ affects the outcome of an evolutionary process. It is now wellknown that certain population structures may enhance or suppress selection in the sense that the probability of a randomly placed mutant to invade the entire population differs from the respective Moran probability obtained for homogeneous mixing (Liberman et al., 2005). We have seen such a divergence 
from the Moran probabilities in Section 4.5 where the VM on a leaderfollower topology has been discussed. The search for paradigmatic network structures which affect the fixation probabilities is still a topic of current research (e.g., Shakarian et al., 2012; Voorhees and Murray, 2013) and the methods developed here, Prop. 4.3.1 in particular, can be directly applied to study not only exit probabilities, but also the pace of mutant fixation.

Thirdly, regarding the idea of relating lumpability to dynamical emergence (Chapter 6), the application to CA is a compelling idea. However, a micro description of the original synchronous CA as Markov chains is challenging as, in principle, all agents can change at a time (notice that original CA are deterministic systems, but that their transitions can nevertheless be encoded in a »transition matrix «). On the other hand, their asynchronous probabilistic counterparts belong precisely to the class of single-step dynamics which we have been concerned with in this thesis. (See, for instance, Schönfisch and de Roos, 1999; Nehaniv, 2004 for the relation between asynchronous and synchronous automata.) In particular, when the probability of choosing a triple $(i, j, k)$ of cells is equal for all triples (complete graph), the micro chain is lumpable with respect to a (macro) description in term of the number of white and respectively black cells (as sensible as such a description might be). Some preliminary computations with the respective macro chains indicate, that the different rules alone, even in a non-localized form, lead to behaviors by which the more complex rules are distinguishable from the simpler ones. This might be useful for classification. While this thesis has been more concerned with the effects of heterogeneity in $\omega$, the systematic study of elementary CA in a homogeneous setting could be a way to understand the contribution of different update rules $\mathbf{u}$ to the dynamic behavior of complex computational models.

Another more general topic that should be addressed in the future is to obtain a more detailed but also more synthetic understanding of the macroscopic effects that may emerge in micro simulation models. One starting point could be a quantification of the range of memory at the macro level in order to gain insight about the microscopic conditions for long-term memory effects that are known to exist in many real world systems from Finance (Cont, 2001) to Biology (Stanley et al., 1994). More generally, under certain circumstances the macro process may undergo dynamical changes in its own structural rules. This fact is referred to as explanatory emergence, a controversial issue in social theory (Giesen, 1987). It can be understood either as a consequence of some external (to the model) inputs or on the basis of deep accelerations of the micro dynamics that in turn bring about the processes of change at the macro level. In both cases this question opens up to new theoretical as well as very interesting practical developments.

All in all, the theory of Markov chain aggregation applied to ABMs provides a useful instrument for the analysis of the link from a microscopic ABM to macroscopic observables and may contribute to our understanding of aggregation and emergence in complex adaptive systems. 


\section{BIBLIOGRAPHY}

Abdel-Moneim, A. M. and Leysieffer, F. W. (1982). Weak Lumpability in Finite Markov Chains. Journal of Applied Probability, 19(3):685 - 691.

Alexander, J. C. (1987). Action and Its Environments. In Alexander, J. C., Giesen, B., Münch, R., and Smelser, N. J., editors, The Micro-Macro Link. University of California press.

Alexander, J. C. and Giesen, B. (1987). From Reduction to Linkage: The Long View of the Micro-Macro Link. In Alexander, J. C., Giesen, B., Münch, R., and Smelser, N. J., editors, The Micro-Macro Link. University of California press.

Alexander, J. C., Giesen, B., Münch, R., and Smelser, N. J., editors (1987). The Micro-Macro Link. University of California press.

Ando, A. and Fisher, F. M. (1963). Near-decomposability, partition and aggregation, and the relevance of stability discussions. International Economic Review, $4(1): 53-67$.

Auger, P., de La Parra, R. B., Poggiale, J.-C., Sánchez, E., and Sanz, L. (2008). Aggregation methods in dynamical systems and applications in population and community dynamics. Physics of Life Reviews, 5(2):79-105.

Auger, P. and Poggiale, J.-C. (1998). Aggregation and emergence in systems of ordinary differential equations. Mathematical and computer modelling, 27(4):121.

Axelrod, R. (1997). The Dissemination of Culture: A Model with Local Convergence and Global Polarization. The Journal of Conflict Resolution, 41(2):203226.

Axelrod, R. (2003). Advancing the Art of Simulation in the Social Sciences. Japanese Journal for Management Information System, Special Issue on AgentBased Modeling, 12(3):1-19. updated article originally published in Simulating Social Phenomena (Berlin: Springer, 1997),21-40.

Axtell, R., Axelrod, R., Epstein, J. M., and Cohen, M. D. (1996). Aligning Simulation Models: A Case Study and Results. Computational and Mathematical Organization Theory, 1(2):123-141.

Ball, R. C., Diakonova, M., and Mackay, R. S. (2010). Quantifying emergence in terms of persistent mutual information. Advances in Complex Systems, 13(03):327-338. 
Banisch, S. Dynamical Emergence in Simple Agent-Based Models. Invited Talk: NESS: Micro-foundations and Systemic Impacts Workshop, Ancona, Italy, 2012.

Banisch, S. Towards a Markov Chain Theory of Aggregation in Agent Based Models. Invited Talk, XIV Arrábida meeting "Caminhos da Complexidade", Portugal, 2013.

Banisch, S. (2013). Analytical assessment of network influence in an opinion model with contrarians. Cultural and opinion dynamics: Modeling, Experiments and Challenges for the future, ECCS 2013 Satellite.

Banisch, S. and Araújo, T. (2012). Who Replaces Whom? Local versus Non-local Replacement in Social and Evolutionary Dynamics. Discontinuity, Nonlinearity, and Complexity, 2(1):57-73.

Banisch, S., Araujo, T., and a, J. L. (2010). Opinion dynamics and communication networks. Advances in Complex Systems, 13(1):95-111. ePrint: arxiv.org/abs/0904.2956.

Banisch, S. and Lima, R. (2012). Markov Projections of the Voter Model. http://arxiv.org/abs/1209.3902.

Banisch, S. and Lima, R. (2013). Markov Chain Aggregation for Simple AgentBased Models on Symmetric Networks: The Voter Model. Submitted to Advances in Complex Systems.

Banisch, S., Lima, R., and Araújo, T. (2012). Agent Based Models and Opinion Dynamics as Markov Chains. Social Networks, 34:549-561.

Banisch, S., Lima, R., and Araújo, T. (2013). Aggregation and Emergence in Agent-Based Models: A Markov Chain Approach. In Gilbert, T., Kirkilionis, M., and Nicolis, G., editors, Proceedings of the European Conference on Complex Systems 2012, Springer Proceedings in Complexity, pages 3-7. Springer International Publishing.

Barr, D. R. and Thomas, M. U. (1977). An Eigenvector Condition for Markov Chain Lumpability. Operations Research, 25(6):1028-1031.

Bärwinkel, K., Schmidt, H.-J., and Schnack, J. (2000). Structure and relevant dimension of the Heisenberg model and applications to spin rings. Journal of Magnetism and Magnetic Materials, 212:240 - 250.

Bedau, M. A. (1997). Weak Emergence. Philosophical Perspectives, 11:375 - 399.

Bedau, M. A. (2003). Downward Causation and the Autonomy of Weak Emergence. Principia Revista Internacional de Epistemologica, 6(1):5-50.

Behrends, E. (2000). Introduction to Markov Chains with Special Emphasis on Rapid Mixing. Friedrick Vieweg \& Son.

Berlekamp, E., Conway, J., and Guy, R. (1982). Winning Ways for your Mathematical Plays, volume 2. Academic. 
Bornholdt, S., Jensen, M. H., and Sneppen, K. (2011). Emergence and decline of scientific paradigms. Physical review letters, 106(5):058701.

Brodbeck, M. (1968). Methodological individualism - definitions and reduction. In Brodbeck, M., editor, Readings in the Philosophy of the Social Sciences, pages 280 - 309. London: Collier-Macmillan.

Buchholz, P. (1994). Exact and Ordinary Lumpability in Finite Markov Chains. J. Appl. Prob., 31(1):59-75.

Buchholz, P. (1995). Hierarchical Markovian models: symmetries and reduction. Performance Evaluation, 22(1):93 - 110. 6th International Conference on Modelling Techniques and Tools for Computer Performance Evalution.

Buchholz, P. (2000). Efficient Computation of Equivalent and Reduced Representations for Stochastic Automata. Computer Systems Science $\mathcal{E}$ Engineering, 15(2):93-103.

Buchholz, P. (2006). Structured Analysis Techniques for Large Markov Chains. In Proceeding from the 2006 Workshop on Tools for Solving Structured Markov Chains, SMCtools '06, New York, NY, USA. ACM.

Burke, C. J. and Rosenblatt, M. (1958). A Markovian Function of a Markov Chain. The Annals of Mathematical Statistics, 29(4):1112 - 1122.

Caruso, F. and Castorina, P. (2005). Opinion dynamics and decision of vote in bipolar political systems. International Journal of Modern Physics C, 16(09):14731487.

Castellano, C., Fortunato, S., and Loreto, V. (2009). Statistical physics of social dynamics. Reviews of Modern Physics, 81(2):591-646.

Castellano, C., Marsili, M., and Vespignani, A. (2000). Nonequilibrium Phase Transition in a Model for Social Influence. Physical Review Letters, 85(16):35363539 .

Chazottes, J.-R., Floriani, E., and Lima, R. (1998). Relative entropy and identification of Gibbs measures in dynamical systems. Journal of Statistical Physics, 90(3-4):697-725.

Chazottes, J.-R. and Ugalde, E. (2003). Projection of Markov Measures May Be Gibbsian. Journal of Statistical Physics, 111(5/6).

Claussen, J. C. and Traulsen, A. (2005). Non-Gaussian fluctuations arising from finite populations: Exact results for the evolutionary Moran process. Physical Review E, 71(2):025101.

Coleman, J. S. (1987). Microfoundations and Macrosocial Behavior. In Alexander, J. C., Giesen, B., Münch, R., and Smelser, N. J., editors, The Micro-Macro Link. University of California press.

Cont, R. (2001). Empirical properties of asset returns: stylized facts and statistical issues. Quantitative Finance, 1(2):223-236. 
Cont, R. and Bouchaud, J.-P. (2000). Herd behavior and aggregate fluctuations in financial markets. Macroeconomic dynamics, 4(2):170-196.

Conte, S. D. and Boor, C. W. D. (1980). Elementary Numerical Analysis: An Algorithmic Approach. McGraw-Hill Higher Education, 3rd edition.

Corcuera, J. M., Nualart, D., and Schoutens, W. (2005). Completion of a Lévy market by power-jump assets. Finance and Stochastics, 9(1):109-127.

Courtois, P.-J. and Semal, P. (1984). Bounds for the Positive Eigenvectors of Nonnegative Matrices and for their Approximations by Decomposition. J. ACM, $31(4): 804-825$.

Cox, J. T. (1989). Coalescing Random Walks and Voter Model Consensus Times on the Torus in Zd. The Annals of Probability, 17(4):1333-1366.

Crutchfield, J. P. and Shalizi, C. R. (1999). Thermodynamic depth of causal states: Objective complexity via minimal representations. Physical Review E, 59(1):275.

Crutchfield, J. P. and Young, K. (1989). Inferring statistical complexity. Physical Review Letters, 63(2):105-108.

Darroch, J. N. and Seneta, E. (1965). On quasi-stationary distributions in absorbing discrete-time finite Markov chains. Journal of Applied Probability, 2(1):88-100.

David, N., Sichman, J. S. a., and Coelho, H. (2005). The Logic of the Method of Agent-Based Simulation in the Social Sciences: Empirical and Intentional Adequacy of Computer Programs. Journal of Artificial Societies and Social Simulation, 8(4):2.

Dayar, T. and Stewart, W. J. (1997). Quasi lumpability, lower-bounding coupling matrices, and nearly completely decomposable Markov chains. SIAM Journal on Matrix Analysis and Applications, 18(2):482-498.

De Oliveira, M., Mendes, J., and Santos, M. (1993). Nonequilibrium spin models with Ising universal behaviour. Journal of Physics A: Mathematical and General, 26(10):2317.

Deffuant, G., Neau, D., Amblard, F., and Weisbuch, G. (2001). Mixing beliefs among interacting agents. Advances in Complex Systems, 3:87-98.

Derisavi, S., Hermanns, H., and Sanders, W. H. (2003). Optimal state-space lumping in Markov chains. Information Processing Letters, 87(6):309 - 315.

Dieckmann, U. and Doebeli, M. (1999). On the origin of species by sympatric speciation. Nature, 400(6742):354-357.

Epstein, J. M. (2006). Remarks on the Foundations of Agent-Based Generative Social Science. In Tesfatsion, L. and Judd, K. L., editors, Handbook of Computational Economics, Vol. 2: Agent-Based Computational Economics, pages 1585-1604. Elsevier B. V.

Epstein, J. M. and Axtell, R. (1996). Growing artificial societies: social science from the bottom up. The Brookings Institution, Washington, DC, USA. 
Filliger, R. and Hongler, M. O. (2008). Lumping Complex Networks. In Lectures and Gallery of Madeira Math Encounters XXXV.

Fisher, R. (1930). The genetical theory of natural selection. The Clarendon Press.

Flajolet, P. and Odlyzko, A. M. (1990). Random Mapping Statistics. In Advances in Cryptology, pages 329-354. Springer Verlag.

Frachebourg, L. and Krapivsky, P. L. (1996). Exact results for kinetics of catalytic reactions. Phys. Rev. E, 53(4):R3009-R3012.

Franceschinis, G. and Muntz, R. R. (1994). Bounds for quasi-lumpable Markov chains. Performance Evaluation, 20(1-3):223 - 243. Performance '93.

Galam, S. (2004). Contrarian deterministic effects on opinion dynamics: "the hung elections scenario". Physica A: Statistical Mechanics and its Applications, $333(\mathrm{C}): 453-460$.

Galán, J. M., Izquierdo, L. R., Izquierdo, S. S., Santos, J. I., del Olmo, R., LópezParedes, A., and Edmonds, B. (2009). Errors and Artefacts in Agent-Based Modelling. Journal of Artificial Societies and Social Simulation, 12(1):1.

Giesen, B. (1987). Beyond reductionism: Four models relating micro and macro levels. In Alexander, J. C., Giesen, B., Münch, R., and Smelser, N. J., editors, The Micro-Macro Link, chapter 15. University of California Press.

Gleeson, J. P. (2011). High-Accuracy Approximation of Binary-State Dynamics on Networks. Phys. Rev. Lett., 107:068701.

Gleeson, J. P. (2013). Binary-State Dynamics on Complex Networks: Pair Approximation and Beyond. Phys. Rev. X, 3:021004.

Gmeiner, P. (2013). Some Properties of Persistent Mutual Information. In Proceedings of the European Conference on Complex Systems 2012, pages 867-876. Springer.

Görnerup, O. and Jacobi, M. N. (2008). A Method for Inferring Hierarchical Dynamics in Stochastic Processes. Advances in Complex Systems, 11(1):1-16.

Görnerup, O. and Jacobi, M. N. (2010). A Method for Finding Aggregated Representations of Linear Dynamical Systems. Advances in Complex Systems, 13(02):199-215.

Grassberger, P. (1986). Toward a quantitative theory of self-generated complexity. International Journal of Theoretical Physics, 25(9):907-938.

Grauwin, S., Goffette-Nagot, F., and Jensen, P. (2010). Dynamic models of residential segregation : an analytical solution. Working Papers 1017, Groupe d'Analyse et de Théorie Economique (GATE), Centre national de la recherche scientifique (CNRS), Université Lyon 2, Ecole Normale Supérieure. 
Grimm, V., Berger, U., Bastiansen, F., Eliassen, S., Ginot, V., Giske, J., GossCustard, J., Grand, T., Heinz, S. K., Huse, G., Huth, A., Jepsen, J. U., Jorgensen, C., Mooij, W. M., Muller, B., Pe'er, G., Piou, C., Railsback, S. F., Robbins, A. M., Robbins, M. M., Rossmanith, E., Ruger, N., Strand, E., Souissi, S., Stillman, R. A., Vabo, R., Visser, U., and Deangelis, D. L. (2006). A standard protocol for describing individual-based and agent-based models. Ecological Modelling, 198:115-126.

Gurvits, L. and Ledoux, J. (2005). Markov property for a function of a Markov chain: A linear algebra approach. Linear Algebra and its Applications, 404(0):85 -117 .

Hales, D., Rouchier, J., and Edmonds, B. (2003). Model-to-Model Analysis. Journal of Artificial Societies and Social Simulation, 6(4).

Harary, F. and Palmer, E. M. (1973). Graphical Enumeration, volume 16. Academic Press.

Heath, J. (2011). Methodological Individualism. In Zalta, E. N., editor, The Stanford Encyclopedia of Philosophy. Spring 2011 edition.

Hegselmann, R. and Krause, U. (2002). Opinion dynamics and bounded confidence: Models, analysis and simulation. Journal of Artificial Societies and Social Simulation, $5(3): 1$.

Hermanns, H. (1999). (Interactive Markov Chains). PhD thesis, FriedrichAlexander Universität Erlangen Nürnberg.

Hermanns, H. and Katoen, J.-P. (2010). The How and Why of Interactive Markov Chains. In Boer, F., Bonsangue, M., Hallerstede, S., and Leuschel, M., editors, Formal Methods for Components and Objects, volume 6286 of Lecture Notes in Computer Science, pages 311-337. Springer Berlin Heidelberg.

Hofbauer, J. and Sigmund, K. (2003). Evolutionary game dynamics. Bulletin of the American Mathematical Society, 40(4):479-519.

Hommes, C. H. (2006). Heterogeneous agent models in economics and finance. In Tesfatsion, L. and Judd, K., editors, Handbook of Computational Economics, Volume 2: Agent-Based Computational Economics, volume 2, pages 1109-1186. Elsevier.

Humphreys, P. (2008). Synchronic and Diachronic Emergence. Minds and Machines, 18(4).

Humphreys, P. (2012). Ising Models: Interpretational and Computational Issues. In Jahrestagung der DPG 2012 in Berlin, Arbeitsgruppe Philosophie der Physik.

Huneman, P. and Humphreys, P. (2008). Dynamical Emergence and Computation: An Introduction. Minds and Machines, 18(4):425-430.

Iwasa, Y., Andreasen, V., and Levin, S. (1987). Aggregation in model ecosystems. I. Perfect aggregation. Ecological Modelling, 37(3):287-302. 
Iwasa, Y., Levin, S. A., and Andreasen, V. (1989). Aggregation in model ecosystems II. Approximate aggregation. Mathematical Medicine and Biology, 6(1):1-23.

Izquierdo, L. R., Izquierdo, S. S., Galán, J. M., and Santos, J. I. (2009). Techniques to Understand Computer Simulations: Markov Chain Analysis. Journal of Artificial Societies and Social Simulation, 12(1):6.

Jacobi, M. N. (2008). A dual eigenvector condition for strong lumpability of Markov chains. CoRR, arxiv.org/abs/0710.1986v2.

Jacobi, M. N. and Görnerup, O. (2009). A spectral method for aggregating variables in linear dynamical systems with application to cellular automata renormalization. Advances in Complex Systems, 12(02):131-155.

James, R. G., Ellison, C. J., and Crutchfield, J. P. (2011). Anatomy of a bit: Information in a time series observation. Chaos, 21(3):7109.

Kemeny, J. G. and Snell, J. L. (1976). Finite Markov Chains. Springer.

Kimura, M. and Weiss, G. H. (1964). The stepping stone model of population structure and the decrease of genetic correlation with distance. Genetics, 49:561576.

Kondrashov, A. S. and Shpak, M. (1998). On the origin of species by means of assortative mating. Proc. R. Soc. Lond. B, 265:2273-2278.

Krapivsky, P. L. and Redner, S. (2003). Dynamics of Majority Rule in Two-State Interacting Spin Systems. Phys. Rev. Lett., 90(23):238701.

Krause, S. M. and Bornholdt, S. (2013). Spin models as microfoundation of macroscopic market models. Physica A: Statistical Mechanics and its Applications.

Laubenbacher, R. C., Jarrah, A. S., Mortveit, H. S., and Ravi, S. S. (2009). Agent Based Modeling, Mathematical Formalism for. In Encyclopedia of Complexity and Systems Science, pages 160-176.

Lazarsfeld, P. and Merton, R. K. (1954). Friendship as a Social Process: A Substantive and Methodological Analysis. In Berger, M., Abel, T., and Page, C. H., editors, Freedom and Control in Modern Society, pages 18-66. New York: Van Nostrand.

LeBaron, B. (2000). Agent-based computational finance: Suggested readings and early research. Journal of Economic Dynamics and Control, 24(5):679-702.

Ledoux, J., Rubino, G., and Sericola, B. (1994). Exact Aggregation of Absorbing Markov Processes using the Quasi-Stationary Distribution. Journal of Applied Probability, 31:626 - 634.

Lerman, K. (2001). Design and Mathematical Analysis of Agent-Based Systems. In Rash, J., Truszkowski, W., Hinchey, M., Rouff, C., and Gordon, D., editors, Formal Approaches to Agent-Based Systems, volume 1871 of Lecture Notes in Computer Science, pages 222-234. Springer Berlin Heidelberg. 
Levin, D. A., Peres, Y., and Wilmer, E. L. (2009). Markov chains and mixing times. American Mathematical Society.

Li, Q., Braunstein, L. A., Wang, H., Shao, J., Stanley, H. E., and Havlin, S. (2012). Non-consensus opinion models on complex networks. CoRR, arxiv.org/abs/1210.0862.

Li, W., editor (1977). Stochastic models in population genetics. Benchmark papers in genetics. Dowden, Hutchinson \& Ross.

Liberman, E., Hauert, C., and Nowak, M. (2005). Evolutionary dynamics on graphs. Nature, 433(7023):312-316.

Liggett, T. M. (1999). Stochastic Interacting Systems: Contact, Voter and Exclusion Processes, volume 324 of Grundlehren der mathematischen Wissenschaften. Springer.

Lindgren, K. and Nordahl, M. G. (1988). Complexity measures and cellular automata. Complex Systems, 2(4):409-440.

Macy, M. W. and Willer, R. (2002). From Factors to Actors: Computational Sociology and Agent-Based Modeling. Annual Review of Sociology, 28(1):143166.

Maruyama, T. (1974). A simple proof that certain quantities are independent of the geographical structure of population. Theoretical population biology, 5(2):148154.

Meila, M. and Shi, J. (2001). A Random Walks View of Spectral Segmentation. In AI and STATISTICS (AISTATS) 2001.

Moran, P. A. P. (1958). Random processes in genetics. In Proceedings of the Cambridge Philosophical Society, volume 54, pages 60-71.

Moretti, P., Liu, S., Baronchelli, A., and Pastor-Satorras, R. (2012). Heterogenous mean-field analysis of a generalized voter-like model on networks. The European Physical Journal B, 85(3):1-6.

Nehaniv, C. L. (2004). Asynchronous automata networks can emulate any synchronous automata network. International Journal of Algebra and Computation, 14(05n06):719-739.

Nielsen, P. H. (2005). Optimal bonus strategies in life insurance: The Markov chain interest rate case. Scandinavian Actuarial Journal, 2005(2):81-102.

Norberg, R. (2006). Dynamic greeks. Insurance: mathematics and economics, $39(1): 123-133$.

Nowak, M. (2006). Evolutionary dynamics: exploring the equations of live. Cambridge, MA: Harvard University Press.

O'Connor, T. and Wong, H. Y. (2012). Emergent Properties. In Zalta, E. N., editor, The Stanford Encyclopedia of Philosophy. Spring 2012 edition. 
Page, S. E. (2012). Aggregation in agent-based models of economies. The Knowledge Engineering Review, 27(02):151-162.

Parsons, T. (1954). Essays in Social Theory. New York: Free press.

Peng, N.-F. (1996). On weak lumpability of a finite Markov chain. Statistics \& Probability Letters, 27(4):313-318.

Pfante, O., Bertschinger, N., Olbrich, E., Ay, N., and Jost, J. (2013). Comparison between different methods of level identification. Advances in Complex Systems (accepted).

Pollicott, M. and Weiss, H. (2001). The Dynamics of Schelling-Type Segregation Models and a Nonlinear Graph Laplacian Variational Problem. Advances in Applied Mathematics, 27(1):17 - 40.

Preis, T., Moat, H. S., and Stanley, H. E. (2013). Quantifying trading behavior in financial markets using Google Trends. Scientific reports, 3.

Pugliese, E. and Castellano, C. (2009). Heterogeneous pair approximation for voter models on networks. EPL (Europhysics Letters), 88(5):58004.

Reynolds, C. W. (1987). Flocks, Herds, and Schools: A Distributed Behavioral Model. In Computer Graphics, pages 25-34.

Roca, C. P., Cuesta, J. A., and Sánchez, A. (2009). Evolutionary game theory: Temporal and spatial effects beyond replicator dynamics. Physics of Life Reviews, 6(4):208-249.

Rogers, L. C. G. and Pitman, J. W. (1981). Markov Functions. The Annals of Probability, 9(4):573-582.

Rosenblatt, M. (1959). Functions of a Markov Process that are Markovian. Journal of Mathematics and Mechanics, 8(4):134-145.

Rubino, G. and Sericola, B. (1989). On Weak Lumpability in Markov Chains. Journal of Applied Probability, 26(3):446 - 457.

Saam, N. (1999). Simulating the Micro-Macro Link: New Approaches to an Old Problem and an Application to Military Coups. Sociological Methodology, 29:4379 .

Schelling, T. (1971). Dynamic Models of Segregation. Journal of Mathematical Sociology, 1(2):143-186.

Schönfisch, B. and de Roos, A. (1999). Synchronous and asynchronous updating in cellular automata. Biosystems, 51(3):123 - 143.

Schuster, P. and Sigmund, K. (1983). Replicator dynamics. Journal of Theoretical Biology, 100(3):533 - 538 .

Schweitzer, F. and Behera, L. (2008). Nonlinear Voter Models: The Transition from Invasion to Coexistence. The European Physical Journal B - Condensed Matter and Complex Systems, 67(3):301-318. 
Schweitzer, P. J. (1984). Aggregation Methods for Large Markov Chains. In Proceedings of the International Workshop on Computer Performance and Reliability, pages 275-286, Amsterdam, The Netherlands, The Netherlands. North-Holland Publishing Co.

Seneta, E. (2006). Non-negative Matrices and Markov Chains (Springer Series in Statistics). Springer, 2nd edition.

Shakarian, P., Roos, P., and A., J. (2012). A review of evolutionary graphs theory with applications to game theory. Biosystems, 107:66-80.

Shalizi, C. R. and Crutchfield, J. P. (2001). Computational mechanics: Pattern and prediction, structure and simplicity. Journal of statistical physics, 104(3-4):817879 .

Shalizi, C. R. and Moore, C. (2003). What Is a Macrostate? Subjective Observations and Objective Dynamics. CoRR, arXiv:cond-mat/0303625.

Shalizi, C. R., Shalizi, K. L., and Haslinger, R. (2004). Quantifying SelfOrganization with Optimal Predictors. Phys. Rev. Lett., 93:118701.

Simon, H. A. (1962). The architecture of complexity. In Proceedings of the American Philosophical Society, pages 467-482.

Simon, H. A. and Ando, A. (1961). Aggregation of variables in dynamic systems. Econometrica: Journal of The Econometric Society, pages 111-138.

Slanina, F. and Lavicka, H. (2003). Analytical results for the Sznajd model of opinion formation. The European Physical Journal B - Condensed Matter and Complex Systems, 35(2):279-288.

Slatkin, M. (1981). Fixation probabilities and fixation times in a subdivided population. Evolution, pages 477-488.

Smith, J. M. (1982). Evolution and the Theory of Games. Cambridge Univ Press.

Sood, V. and Redner, S. (2005). Voter Model on Heterogeneous Graphs. Phys. Rev. Lett., 94(17):178701.

Squazzoni, F. (2008). The Micro-Macro Link in Social Simulation. Sociologica, 1.

Stanley, H., Buldyrev, S., Goldberger, A., Goldberger, Z., Havlin, S., Mantegna, R., Ossadnik, S., Peng, C.-K., and Simons, M. (1994). Statistical mechanics in biology: how ubiquitous are long-range correlations? Physica A: Statistical Mechanics and its Applications, 205(1):214-253.

Stark, H.-U., Tessone, C. J., and Schweitzer, F. (2008). Decelerating microdynamics can accelerate macrodynamics in the voter model. Physical review letters, 101(1):018701.

Steels, L. (1997). Self-Organizing Vocabularies. In Langton, C. and Shimohara, T., editors, Artificial Life V: Proceeding of the Fifth International Workshop on the Synthesis and Simulation of Living Systems, pages 179-184. The MIT Press. 
Stewart, W. J. (2009). Probability, Markov chains, queues, and simulation: the mathematical basis of performance modeling. Princeton University Press.

Sumita, U. and Rieders, M. (1989). Lumpability and time reversibility in the aggregation-disaggregation method for large Markov chains. Stochastic Models, $5(1): 63-81$.

Symons, J. (2008). Computational Models of Emergent Properties. Minds and Machines, 18(4):475-491.

Takacs, C. (2006). On the fundamental matrix of finite state Markov chains, its eigensystem and its relation to hitting times. Mathematica Pannonica, 17(2):183193.

Taylor, P. D. and Jonker, L. B. (1978). Evolutionary stable strategies and game dynamics. Mathematical biosciences, 40(1):145-156.

Tesfatsion, L. and Judd, K. L. (2006). Handbook of Computational Economics, Volume 2: Agent-Based Computational Economics. North-Holland Publishing Co., Amsterdam, The Netherlands, The Netherlands.

Theil, H. (1965). Linear aggregation of economic relations. Contributions to economic analysis. North-Holland Pub. Co.

Touzene, A. (2013). A new parallel algorithm for solving large-scale Markov chains. The Journal of Supercomputing, pages 1-15.

Traulsen, A., Claussen, J. C., and Hauert, C. (2005). Coevolutionary Dynamics: From Finite to Infinite Populations. Phys. Rev. Lett., 95:238701.

Vazquez, F. and Eguíluz, V. M. (2008). Analytical solution of the voter model on uncorrelated networks. New Journal of Physics, 10(6):063011.

Vilela Mendes, R., Lima, R., and Araújo, T. (2002). A process-reconstruction analysis of market fluctuations. International Journal of Theoretical and Applied Finance, 5(08):797-821.

von Neumann, J. (1951). The general and logical theory of automata, pages 1-41. Wiley, Pasadena CA.

Voorhees, B. (2013). Birth-death fixation probabilities for structured populations. Proceedings of the Royal Society A: Mathematical, Physical and Engineering Science, 469(2153).

Voorhees, B. and Murray, A. (2013). Fixation probabilities for simple digraphs. Proceedings of the Royal Society A: Mathematical, Physical and Engineering Science, $469(2154)$.

Weber, M. (1978). Economy and Society. London, University of California Press, 29, Berkley, Los Angeles. Collected translation from different original work, from 1909 to 1920.

Wilensky, U. and Rand, W. (2007). Making Models Match: Replicating an AgentBased Model. Journal of Artificial Societies and Social Simulation, 10(4):2. 
Wimsatt, W. (2000). Emergence as Non-Aggregativity and the Biases of Reductionisms. Foundations of Science, 5(3):269-297.

Wimsatt, W. C. (1986). Forms of aggregativity, pages 259 - 291. Reidel, Dordrecht.

Wimsatt, W. C. (2006a). Aggregate, composed, and evolved systems: Reductionistic heuristics as means to more holistic theories. Biology \& Philosophy, 21(5):667-702.

Wimsatt, W. C. (2006b). Reductionism and its heuristics: Making methodological reductionism honest. Synthese, 151(3):445-475.

Wolfram, S. (1983). Statistical mechanics of cellular automata. Reviews of Modern Physics, 55(3):601-644.

Wolfram, S. (1994). Cellular automata and complexity: collected papers, volume 1. Addison-Wesley Reading.

Wolfram, S. (2002). A new kind of science. Wolfram Media Inc., Champaign, Ilinois, US, United States.

Wright, S. (1932). The roles of mutation, inbreeding, crossbreeding, and selection in evolution. Proceedings of the Sixth International Congress on Genetics.

Wright, S. (1943). Isolation by Distance. Genetics, 28:114-138. 